\title{
Analysis of the JA-lle-independent function of COI1 in Arabidopsis thaliana upon infection with Verticillium longisporum
}

\author{
Dissertation \\ for the award of the degree \\ "Doctor rerum naturalium" \\ of the Georg-August-Universität Göttingen
}

within the doctoral program Biology

of the Georg-August University School of Science (GAUSS)

\author{
submitted by \\ Louisa Ulrich \\ from Braunschweig, Germany
}

Göttingen 2021 


\section{Thesis Committee}

Prof. Dr. Christiane Gatz, Plant Molecular Biology and Physiology, Albrecht-von-HallerInstitut, Georg-August-Universität Göttingen

Prof. Dr. Petr Karlovsky, Molecular Phytopathology and Mycotoxin Research, Georg-AugustUniversität Göttingen

Dr. Corinna Thurow, Plant Molecular Biology and Physiology, Albrecht-von-Haller-Institut, Georg-August-Universität Göttingen

\section{Members of the Examination Board}

First Reviewer

Prof. Dr. Christiane Gatz, Plant Molecular Biology and Physiology, Albrecht-von-HallerInstitut, Georg-August-Universität Göttingen

Second Reviewer

PD Dr. Thomas Teichmann, Plant Cell Biology, Albrecht-von-Haller-Institut, Georg-AugustUniversität Göttingen

\section{Further Members of the Examination Board:}

Prof. Dr. Gerhard Braus, Molecular Microbiology and Genetics, Georg-August-Universität Göttingen

Prof. Dr. Kai Heimel, Molecular Microbiology and Genetics, Georg-August-Universität Göttingen

PD Dr. Till Ischebeck, Plant Biochemistry, Albrecht-von-Haller-Institut, Georg-AugustUniversität Göttingen

PD Dr. Marcel Wiermer, Molecular Biology of Plant-Microbe Interactions, Albrecht-von-HallerInstitut, Georg-August-Universität Göttingen

Date of oral examination: 9/9/2021 




\section{Contents}

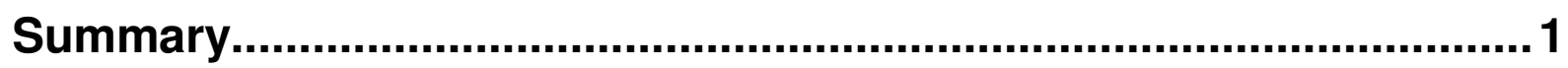

List of Abbreviations .....................................................................

I. General Introduction .....................................................................

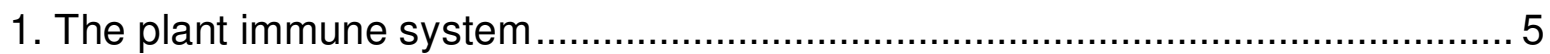

1.1 PAMP-triggered immunity and effector-triggered immunity ...............................................

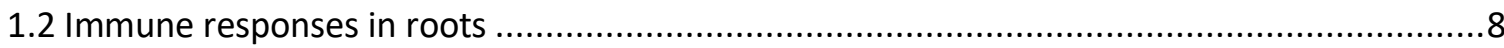

2. Hormone-mediated signalling pathways ................................................... 10

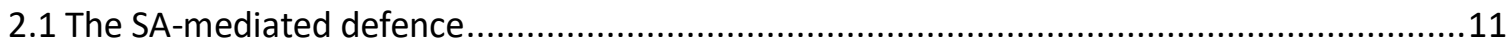

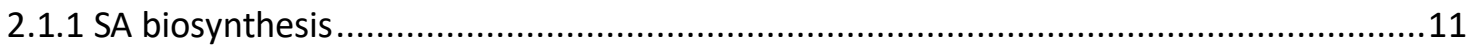

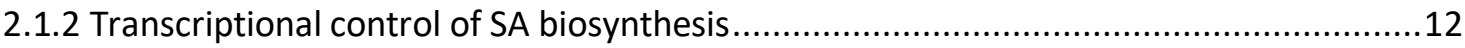

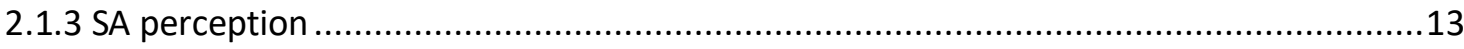

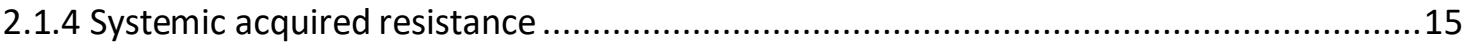

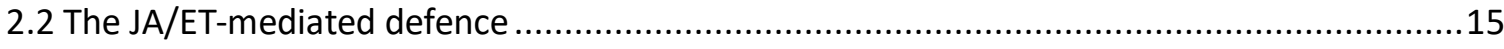

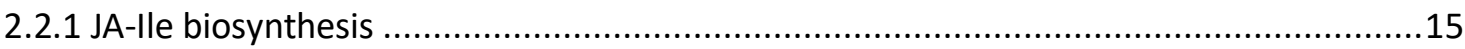

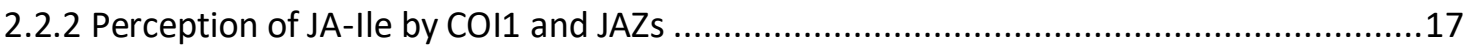

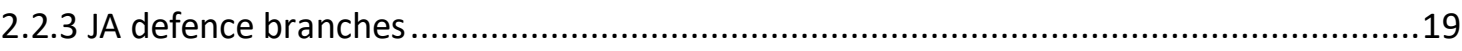

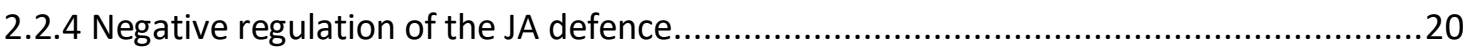

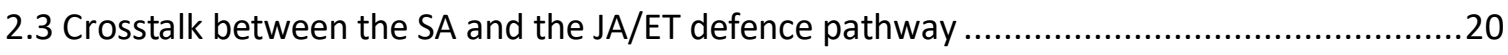

3. The vascular pathogen Verticillium longisporum .......................................... 21

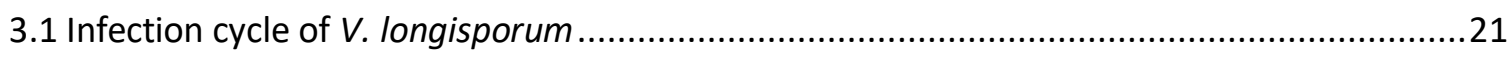

3.2 Disease control measures against $V$. longisporum are insufficient.....................................23

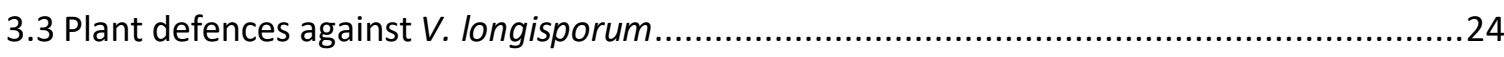

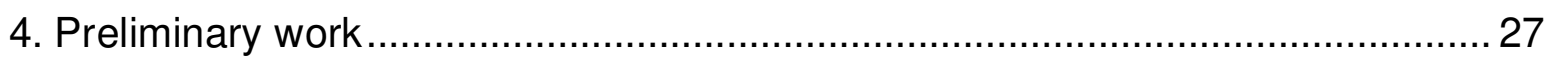

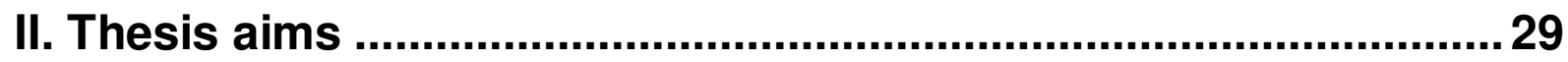

III. Article 1: The jasmonoyl-isoleucine receptor CORONATINE INSENSITIVE1 suppresses defense gene expression in Arabidopsis roots independently of its ligand ...................................................30

Detailed contributions by the PhD candidate:........................................................................30

IV. Article 2: Induction of salicylic acid-related defence genes in Arabidopsis roots upon infection with Verticillium longisporum requires transcription factor SARD1 and the inactivation of COI1mediated repression ...................................................................... 72

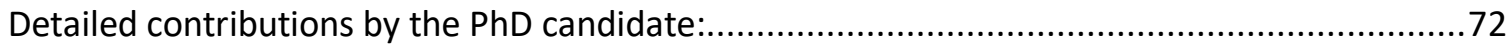

V. Additional Data to Articles ...........................................................133 
1. Additional Data

1.1 The role of $A T 3 G 05770$ in facilitating susceptibility to $V$. longisporum could not be determined

1.2 WRKY49 is not a susceptibility gene enabling effective infection by $V$. longisporum .134

1.3 Neither HDA6 nor SARD1 interacts with COI1 in yeast 134

2. Methods for Additional Data to Articles 138

VI. General Discussion

1. Responses to $V$. longisporum infection differ at early and late time points

2. Regulation of COI1-mediated gene repression differs from COI1's mode of action in canonical JA signalling.

3. Basal upregulation SARD1/CBP60g-dependent defence genes in roots is not the reason for tolerance of coi1

4. The effectiveness of SARD1-regulated defences seems to differ against $V$. longisporum and $V$. dahliae

5. Outlook 149

VII. References

VIII. Appendix 165

IX. Acknowledgements 168 


\section{Summary}

CORONATINE INSENSITIVE 1 (COI1) perceives the plant hormone jasmonoyl-isoleucine (JAIle) together with proteins of the JASMONATE ZIM-domain (JAZ) family. JA-lle induces signalling cascades in defence and developmental processes. It has been shown that in Arabidopsis thaliana, COl1 without its ligand conveys susceptibility to the soil-born vascular pathogen Verticillium longisporum. Grafting experiments have shown that presence of COI1 in roots mediates susceptibility to the pathogen. Root transcriptome analysis has revealed that a number of salicylic acid defence-associated genes are constitutively expressed in coi1. The observation that COI1 acts as a JA-Ile-independent repressor of root gene expression led us to postulate that this novel COI 1 function operates independently of the canonical JA signalling machinery.

In this thesis, we show that coi1 plants complemented with a COI1 protein, that was severely impaired in its interaction with $\mathrm{JAZ}$ proteins $\left(\mathrm{COI} 1_{\mathrm{AA}}\right)$, were compromised in wound-induced induction of the JA-signalling marker gene VEGETATIVE STORAGE PROTEIN 2 (VSP2). Moreover, COI1 $1_{\mathrm{AA}}$ could not restore fertility in sterile coi1 plants. In contrast, $\mathrm{COI} 1_{\mathrm{AA}}$ was able to repress gene expression in roots. Hence, in roots, COl1 has a second function other than its role in JA-Ile perception, in which it acts as a suppressor of defence gene expression independently of JA-lle and most likely independently of JAZ proteins. We furthermore show that after infection with $V$. longisporum, approximately half of the COl1-repressed genes in roots are induced to similar levels as in coi1. We hence postulate that COl1-mediated repression is inactivated upon infection with $V$. longisporum leading to induction of these genes. Gene induction requires the transcription factor SYSTEMIC ACQUIRED RESISTANCE 1 (SARD1) which is itself repressed by COI1. Equally, constitutive expression of genes in coi1 was abolished by mutations in SARD1 and its close homologue CALMODULIN BINDING PROTEIN 60-LIKE G. In contrast, overexpression of SARD1 in wild-type roots did not lead to activation of gene expression, likely because the repressive effect of COl1 on gene expression could not be overcome. The repressor function of COI1 was only observed in roots and not in shoots. As roots need to balance perception of microbe-associated molecular patterns with maintaining an intact rhizosphere, we speculate that COI1 acts as a regulator of the onset of defence responses in roots. 


\title{
List of Abbreviations
}

\author{
AIM1 - ABNORMAL INFLORESCENCE MERISTEM 1 \\ AOC - ALLENE OXIDE CYCLASE \\ AOS - ALLENE OXIDE SYNHASE \\ ASK1 - ARABIDOPSIS SKP1-LIKE 1
}

A. thaliana - Arabidopsis thaliana

bHLH - basic helix-loop-helix

B. napus - Brassica napus

CAMTA - Calmodulin-binding transcription factor

CBP60g - CALMODULIN-BINDING PROTEIN 60G

COI1 - CORONATINE INSENSITIVE 1

COI1 $_{\text {AA }}$ - COl1 protein with amino acid residues Glu203 and Tyr302 changed to Alanine

CUL1 - CULLIN 1

CYP - CYTOCHROME P450 family protein

Cys - cysteine

DAMP - damage-associated molecular pattern

DLO1 - DMR6-LIKE OXYGENASE 1

DNA - deoxyribonucleic acid

dpi - days past inoculation

ECS1 - Pathogen-inducible protein CXC75

EDS5 - ENHANCED DISEASE SUSCEPTIBILITY 5

EIL1 - EIN3-LIKE 1

EIN3 - ETHYLENE INSENSITIVE 3

ET - ethylene

ETI - effector-triggered immunity

flg22 - conserved 22 amino acid sequence of flagellin

FLS2 - FLAGELLIN SENSITIVE 2

F. oxysporum - Fusarium oxysporum

GLP - Germin-like protein

Glu - Glutamic acid 
HA-tag - Human influenza hemagglutinin tag

HR - hypersensitive response

ICS1 - ISOCHORISMATE SYNTHASE 1

IGs - indole glucosinolates

JA - jasmonic acid

JA-lle - jasmonoyl isoleucine

JAZ - JASMONATE ZIM-domain

jazD - jaz decuple mutant

LRR - leucine-rich repeat

LTP4.4 - LIPID TRANSFER PROTEIN 4.4

MAMP - microbe-associated molecular-pattern

MAPK - mitogen-activated protein kinase

MED25 - MEDIATOR 25

MeJA - methyl jasmonate

NAC - No apical meristem (NAM), Arabidopsis transcription activation factor (ATAF), Cup- shaped cotyledon (CUC)

NHP - N-hydroxypipecolic acid

NINJA - NOVEL INTERACTOR OF JAZ

NLP - Necrosis and ethylene-inducing peptide 1 (Nep1)-like protein

NLR - nucleotide-binding (NB) leucine-rich repeat (LRR) protein

NPR1 - NON-EXPRESSER OF PATHOGENESIS-RELATED GENES 1

NPR3 - NPR1-LIKE PROTEIN 3

OPR3 - OPDA REDUCTASE 3

ORA59 - OCTADECANOID-RESPONSIVE ARABIDOPSIS 59

PAL - PHENYLALANINE AMMONIA-LYASE

PAMP - pathogen-associated molecular pattern

PBS3 - avrPphB SUSCEPTIBLE 3

PDF1.2 - PLANT DEFENSIN 1.2

Pep1 - plant elicitor peptide 1

PGM - PHOSPHOGLYCERATE MUTASE

PRLIP2 - PATHOGENESISRELATED LIPASE 2 
PRR - pathogen recognition receptors

PTI - PAMP-triggered immunity

RBX1 - RING-BOX 1

RLK - receptor-like kinase

RLP - receptor-like protein

RNA - ribonucleic acid

RNA-seq - RNA sequencing

ROS - reactive oxygen species

SA - salicylic acid

SAR - Systemic acquired resistance

SARD1 - SAR-DEFICIENT 1

SCFCOI1 - SKP1/CULLIN/F-BOX E3 ligase complex formed with COI1

sGFP - superfolder GREEN FLUORESCENT PROTEIN

sid2 - salicylic acid induction-deficient 2

TPL - TOPLESS

Tyr - Tyrosine

V. dahliae -Verticillium dahliae

V. Iongisporum - Verticillium longisporum

VND7 - VASCULAR-RELATED NAC DOMAIN 7

VSP2 - VEGETATTIVE STORAGE PROTEIN 2

$\mathrm{WT}_{\text {aos }}$ - wild-type plant originating from the segregating offspring of heterozygous aos population

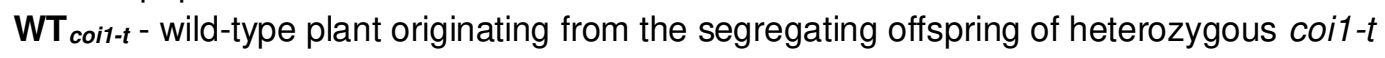
population

ZIM - ZINC-FINGER EXPRESSED IN INFLORESCENCE MERISTEM 


\section{General Introduction}

\section{The plant immune system}

\subsection{PAMP-triggered immunity and effector-triggered immunity}

Plants have a sessile lifestyle and face a myriad of pathogenic microorganisms. Nevertheless, due to carefully regulated and broadly effective defence responses, plant disease is the exception and not the norm. Instead of having mobile defence cells, plants rely on the reaction of each cell and the propagation of signals from infection sites (Jones and Dangl, 2006). Plants have several layers of defence that constitute potent protection against infection by potential pathogens.

At the forefront of plant defences are structural barriers such as waxy cuticles and cell walls, limiting access of prospective pathogens to inner cells (Malinovsky et al., 2014; Serrano et al., 2014). At sites of penetration, plants can reinforce or create new barriers by deposition of structural elements such as callose or lignin to restrict pathogen entry or spread (Lee et al., 2019; Wang et al., 2021).

In opposition, pathogens attack these structural barriers with enzymes degrading their individual components (Kubicek et al., 2014). If the plant's barriers are breached, a second layer of defence senses the imminent danger. On the surface of plant cell membranes pathogen recognition receptors (PRRs) detect conserved molecules associated with microorganisms, so called pathogen-associated molecular patterns (PAMPs) or more broadly and accurately microbe-associated molecular-patterns (MAMPs). PRRs transduce this information to the inside of the cell (Jones and Dangl, 2006). PRRs can be divided into receptor-like kinases $(R L K)$ and receptor-like proteins $(R L P)$. RLKs possess a cytosolic kinase domain for intracellular signal transduction upon ligand binding outside the cell. RLPs lack an intracellular kinase domain and rely on interaction partners for internal signal transduction (Macho and Zipfel, 2014). 
An example of a MAMP is flagellin, the main constituent of bacterial flagella (Gómez-Gómez and Boller, 2002). In fact, a conserved 22 amino acid sequence of flagellin, flg22, is sufficient to elicit an immune response after binding to the PRR FLAGELLIN SENSITIVE 2 (FLS2) (Felix et al., 1999; Chinchilla et al., 2006). Another example of MAMPs are Necrosis and ethyleneinducing peptide 1 (Nep1)-like proteins (NLPs), small proteins found in diverse microbes across kingdoms (Pemberton and Salmond, 2004; Oome et al., 2014). Again, a conserved small fragment of NLPs is sufficient for recognition by the PRR RLP23 (Albert et al., 2015). Special about these NLPs is that they trigger plant defence responses but at the same time contribute to a pathogen's virulence and can be cytotoxic (Qutob et al., 2006).

In addition to non-self-recognition, plants can sense pathogen attack by self-recognition. Receptors on the cell surface can sense damage-associated molecular patterns (DAMPs). These are molecules like cell wall fragments, peptides or nucleotides that are secreted by attacked cells or released from the cytosol of damaged cells into the intercellular space (Hou et al., 2019). A well-studied example of a DAMP is plant elicitor peptide 1 (Pep1), a 23 aminoacid long peptide derived from PRECURSOR OF PEPTIDE 1 (PROPEP1) (Huffaker et al., 2006). Pep1 is perceived by PEP RECEPTOR 1 (PEPR1) and PEPR2 on the plasma membrane, leading to downstream immune responses in the cell. Cleavage of PROPEP1 is achieved by the $\mathrm{Ca}^{2+}$-dependent caspase METACASPASE4 (MC4), the mode of transport to the apoplast is still elusive though (Hander et al., 2019).

Downstream of MAMP and DAMP recognition, the information is passed on via signalling cascades. Cumulatively, the following broad responses aimed to fight off pathogen attack are termed PAMP-triggered immunity (PTI) (Jones and Dangl, 2006). Early responses upon MAMP/DAMP sensing are cytosolic calcium influxes, reactive oxygen species (ROS) bursts and the activation of mitogen-activated protein kinases (MAPKs) (Zhang and Zhou, 2010; Bigeard et al., 2015; Zipfel and Oldroyd, 2017). Calcium is an important second messenger that activates further signal transducers such as calcium-dependent protein kinases (CDPKs) and transcription factors (Gao et al., 2014; Bigeard et al., 2015). ROS production is mediated by respiratory burst oxidase homologs $(\mathrm{RBOHs})$ and ROS act as another type of second 
messengers that are also thought to possess antimicrobial properties themselves (Kadota et al., 2015). The MAPK signal transduction cascade leads to transcriptional reprogramming by targeting transcription factors of the WRKY family resulting, e.g. in the production of the phytoalexin camalexin (Kim and Zhang, 2004; Mao et al., 2011). Pathogen detection also triggers the production of phytohormones that regulate distinct branches of defence responses (Bari and Jones, 2009). Hormone-mediated defence signalling pathways will be discussed in more detail in the next chapter (2. Hormone-mediated signalling pathways).

Pathogens can secrete effectors to supress PTI, leading to so called effector triggered susceptibility (ETS) (Jones and Dangl, 2006). In turn, plants have another third layer of defence. Effector-triggered immunity $(\mathrm{ETI})$ aims at disarming pathogens by recognition of such effectors (Dangl and Jones, 2001). The receptors for such effectors, nucleotide-binding (NB) leucine-rich repeat (LRR) proteins (NLRs), are found intracellularly (Dangl and Jones, 2001). Triggering of ETI leads to similar but stronger and faster responses than $\mathrm{PTI}$, along with a form of programmed cell death, termed the hypersensitive response (HR) (Thordal-Christensen, 2020). HR is initiated by the plant at the point of penetration to restrict pathogen spread (BalintKurti, 2019).

Despite the overlapping defence outputs of $\mathrm{PTI}$ and $\mathrm{ETI}$, the two responses were long seen as two separate tiers of immunity. Recent studies have changed the understanding of the interplay between PTI and ETI using systems that allow induction of ETI without PTI (Ngou et al., 2021; Yuan et al., 2021). When only ETI is triggered, components of PTI accumulated in the cell (Ngou et al., 2021; Yuan et al., 2021). ETI is hence replenishing PTI constituents to strengthen PTI weakened by effectors. Conversely, HR in ETI is strongly enhanced by activation of PRRs (Ngou et al., 2021). The model of PTI and ETI as separate responses thus has to be revised in favour of a mutually potentiated interplay of PTI and ETI in plant defence. 


\section{General Introduction}

\subsection{Immune responses in roots}

Roots are constantly submerged in an environment full of MAMPs. However, our current knowledge of immunity is mostly based on the study of aerial plant parts. Great progress has been made recently in the investigation of root specific immune response elicitation.

Roots have been shown to perceive and react to MAMPs via PRRs. flg22 elicitation of roots leads to induction of defence genes like MYB DOMAIN PROTEIN 51 (MYB51) and WRKY11 and induces production of camalexin and callose deposition (Millet et al., 2010). Other MAMPS and DAMPs, like chitin, Pep1 and NLPs are also recognised by roots (Poncini et al., 2017; Zhou et al., 2020).

In contrast to shoots, root immune responses are highly dependent on cell types and developmental stages. flg22 treatment of seedling roots only elicits defence responses in the elongation zone, whereas chitin induces defences in cells of the mature zones of seedling roots (Millet et al., 2010). In line with this, it has been shown that receptor expression varies in different tissues, for example, FLS2 is higher expressed in lateral roots, as they are an easy entry point for pathogens (Beck et al., 2014).

Moreover, not all cells have the same ability to respond to elicitors, even if they had the required PRR. Emonet et al. (2021) showed that ectopically expressing FLS2 in the vascular meristem of Arabidopsis seedlings did not lead to induction of defences upon treatment with flg22. RichGriffin et al. (2020) showed that differential responses in different cells are underpinned by specific signalling networks in different tissues. Responses to flg22 and Pep1 are differentially regulated by specific pairs of transcription factor families in different cell types in roots. Treatment of epidermal cells with flg22 induced immune responses specifically regulated by WRKY12, 18, 36, 45 together with AT-hook motif nuclear localized protein (AHL) transcription factors $A H L 12,20,25$. In contrast, Pep1 treatment induced cascades coordinated by WRKY12, 18, 36, 45 and No apical meristem (NAM), Arabidopsis transcription activation factor (ATAF), Cup-shaped cotyledon (CUC) (NAC) family transcription factors ANAC46, 55, 55_2, 58 in epidermal cells. Cortex cells had different signalling networks upon the same flg22 
treatment with response curated by WRKY12, 38, 45 and Arabidopsis thaliana homeobox (ATHB) transcription factors ATHB15 and 51 (Rich-Griffin et al., 2020).

Elicitation with MAMPs induces calcium signalling with the $\mathrm{Ca}^{2+}$ wave emerging from the elongation zone into other root zones, spreading the signal even to cells without PRRs themselves (Keinath et al., 2015; Emonet et al., 2021). This might aid roots in balancing crucial danger perception with overreactions in the omnipresent MAMP environment (Keinath et al., 2015; Emonet et al., 2021). Additionally, it has been shown that roots have another way of balancing responses instead of spatial distribution of PRRs and cell type specific responses. In accordance with earlier observations, Zhou et al. (2020) showed that responses to most MAMPs in seedlings were restricted to undifferentiated root zones. However, they observed highly localised defence responses in the differentiated zone where damage occurred, e.g. at sites of lateral root emergence. Upon localised laser ablation, cells in the differentiated zones became responsive to MAMPs. This coincided with the upregulation of PRR receptor transcription in damaged cells (Zhou et al., 2020). However, as triggering of differentiated root zones with a mix of DAMPs and MAMPs together did not lead to defence response induction, other so far unknown signals accompanying damage play a role in defence initiation. Challenging roots with the commensal bacterium Pseudomonas protegens $\mathrm{CHAO}$ did not cause defence reactions in the differentiated root zone, but challenge with the root damage causing pathogen Ralstonia solanacearum GMI1000 did lead to induction of defence responses in the differentiated root (Zhou et al., 2020). Hence, differentiated roots possess a regulation mechanism to balance tolerating non-harmful or beneficial microbes and defending against harmful pathogens. In line with this, the DAMP Pep1 elicits much stronger responses in roots compared to MAMPS, suggesting that DAMPs might be the more reliable danger signal for roots in an environment crowded with MAMPs (Poncini et al., 2017).

For many abiotic stresses and for nodulation regulation extensive root to shoot signalling has been demonstrated (Shabala et al., 2015; Ko and Helariutta, 2017). Signal propagation is achieved via $\mathrm{Ca}^{2+}$ and ROS waves, electrical signalling, and the transport of hormones, secondary metabolites, proteins, peptides and RNAs in the vasculature (Lucas et al., 2013; 
Shabala et al., 2015). Reports on defence-related root to shoot signalling are less plentiful. Wang et al. (2019) describe a synergistic system of ROS and electrical signalling between roots and shoots in Solanum lycopersicum in the jasmonic acid (JA)-mediated defence against the root knot nematode Meloidogyne incognita (for JA defences see 2.2 The JA/ET-mediated defence) (Wang et al., 2019). Infection of roots with M. incognita leads to accumulation of JA in roots and shoots. Grafting experiments showed that resistance to $M$. incognita was principally depending on JA synthesis from the shoot and not the root (Wang et al., 2019). After infection the root sends interdependent $\mathrm{H}_{2} \mathrm{O}_{2}$ and electrical signals up the stem which lead to the activation of MPK1 and MPK2 in leaves. Activation of MAPKs initiates the biosynthesis of $\mathrm{JA}$, which is then transported down to roots to mediate defences against $M$. incognita.

\section{Hormone-mediated signalling pathways}

Hormone-mediated defence responses are complex networks of signalling cascades. Each hormone induces a different signalling pathway leading to downstream transcriptional reprogramming. Different hormone pathways can synergistically or antagonistically modify the plant defence output (Tsuda et al., 2009; Mine et al., 2018; Aerts et al., 2021). Two major hormone-mediated defence pathways are the salicylic acid (SA)-mediated defence and the jasmonic acid (JA)-mediated defence response. In general, the SA defence pathway is effective against biotrophs; pathogens that derive nutrients from living hosts (Glazebrook, 2005). The JA defence pathway is generally launched in response to wounding and herbivory and also converges with the ethylene (ET) pathway to generate defence output against necrotrophic pathogens that kill host plants and feed off the dead tissue (Glazebrook, 2005). 


\subsection{The SA-mediated defence}

\subsubsection{SA biosynthesis}

Biosynthesis of SA can be achieved through two pathways; the Isochorismate Synthase (ICS) pathway and the Phenylalanine Ammonia-Lyase (PAL) pathway. Figure 1 presents an overview of both biosynthesis pathways. Differences in the importance of both pathways for SA production exist between different plant species. In Arabidopsis, the ICS pathway is crucial for biosynthesis of pathogen-triggered SA accumulation (Wildermuth et al., 2001). Chorismate is the starting point of both pathways which is a product of the shikimate pathway in plastids (Eberhard et al., 1993; Wildermuth et al., 2001).

The ICS pathways starts in plastids by conversion of chorismate to isochorismate (Strawn et al., 2007). Two enzymes can do this conversion, ISOCHORISMATE SYNTHASE 1 (ICS1) and ICS2, with ICS1 being of far greater importance for SA production in Arabidopsis (Wildermuth

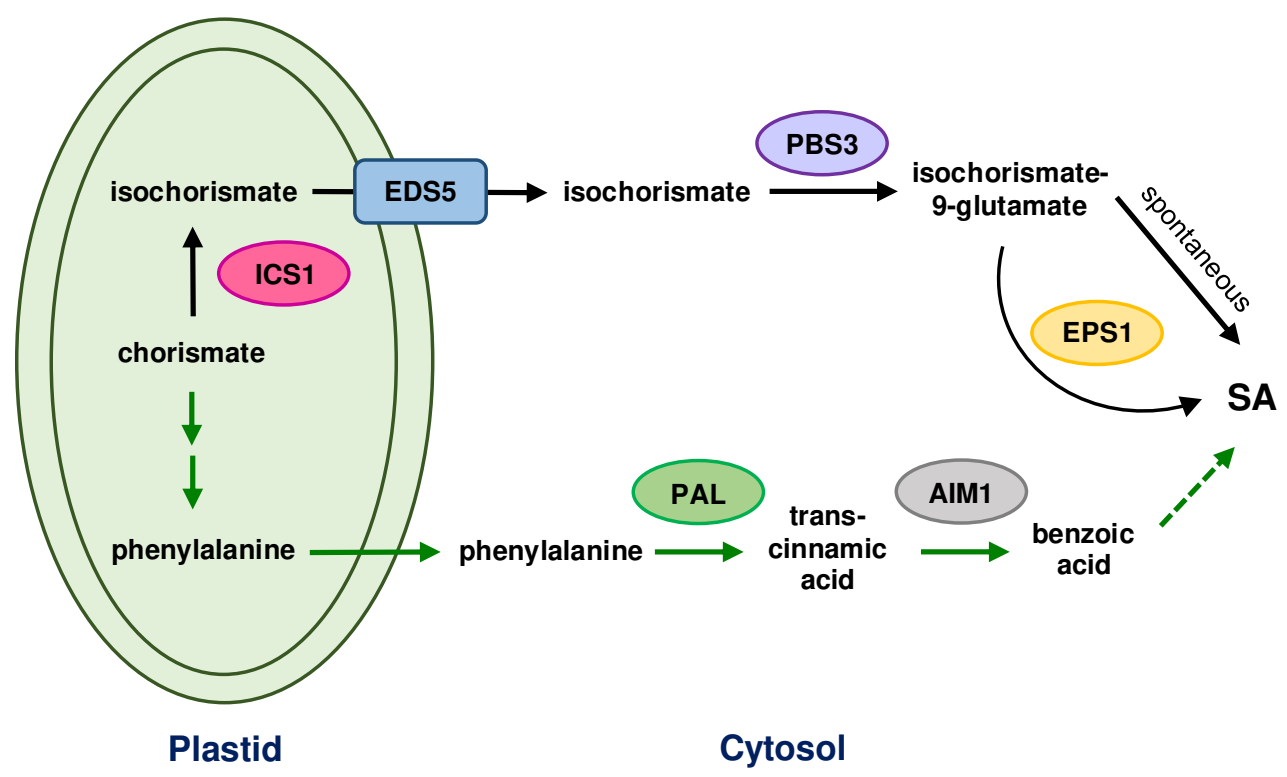

\section{Figure 1. Overview of SA biosynthesis through the ICS (top pathway) and the PAL pathway} (bottom pathway).

Both pathways start from chorismate in the plastid. The ICS pathway (black arrows) involves conversion of chorismate via ICS1 to isochorismate, which is exported from the plastid, most likely through EDS5. In the cytosol, isochorismate is further modified by PBS3. Spontaneous decomposition leads to the end product SA. EPS1 can additionally boost this final reaction. In the PAL pathway (green arrows), chorismate is converted to phenylalanine which is further processed in the cytosol by PAL and AIM1 to benzoic acid. Enzymes facilitating the final conversion to SA are unknown (dotted arrow). Modified from Huang et al. (2020). 
et al., 2001; Garcion et al., 2008; Macaulay et al., 2017; Yokoo et al., 2018). Isochorismate is then transported to the cytosol, most likely via the membrane transporter ENHANCED DISEASE SUSCEPTIBILITY 5 (EDS5) (Serrano et al., 2013; Yamasaki et al., 2013; Rekhter et al., 2019). In the cytosol, avrPphB SUSCEPTIBLE 3 (PBS3) converts isochorismate to isochorismate-9-glutamate, which is then converted to SA by the acetyltransferase ENHANCED PSEUDOMONAS SUSCEPTIBILITY 1 (EPS1) or by spontaneous decomposition (Rekhter et al., 2019; Torrens-Spence et al., 2019).

In Arabidopsis, the PAL pathway only plays a minor role in pathogen-triggered accumulation of SA. In plastids, chorismate is converted to phenylalanine, which is exported into the cytosol, where PAL converts it to trans-cinnamic acid (Mobley et al., 1999; Cho et al., 2007; Lefevere et al., 2020). Trans-cinnamic acid is further converted into benzoic acid by ABNORMAL INFLORESCENCE MERISTEM 1 (AIM1) and finally to SA via yet unknown enzymes (Ribnicky et al., 1998; Richmond and Bleecker, 1999; Zhang and Li, 2019).

\subsubsection{Transcriptional control of SA biosynthesis}

Since the SA pathway is a major defence response, SA biosynthesis has to be tightly controlled. Foremost, SYSTEMIC ACQUIRED RESISTANCE DEFICIENT 1 (SARD1) and CALMODULIN-BINDING PROTEIN 60G (CBP60g) are recognised as master regulators of ICS1 induction. In sard1 cbp60g double mutants pathogen-triggered SA accumulation is almost completely abolished (Wang et al., 2009; Zhang et al., 2010; Wang et al., 2011). ICS1 is not the only SA biosynthesis gene targeted by SARD1 and CBP60g. PBS3 and EDS5 are also positively regulated by these transcription factors (Sun et al., 2015). SARD1 and CBP60g, therefore, positively regulate all major enzymes of the SA biosynthesis pathway. In addition, a number of WRKY transcription factors, WRKY 8, 28, 46 and 48, and two transcription factors of the Teosinte branched1/ Cycloidea/ Proliferating cell factor (TCP) family, TCP8 and TCP9, have been shown to contribute to positive regulation of ICS1 expression (Gao et al., 2013) 
Negative regulators of SA biosynthesis include three transcription factors of the NAC family, ANAC019, ANAC055, and ANAC072, which target ICS1 during JA-crosstalk (see section 2.3 Crosstalk between the SA and the JA/ET defence pathway) (Zheng et al., 2012). CBP60a, WRKY18 and WRKY40 also negatively regulate the ICS1 promoter (Truman et al., 2013; Birkenbihl et al., 2017). Moreover, WRKY18 and WRK40 negatively regulate EDS5 and PBS3, therefore downregulating expression of all important enzymes of SA biosynthesis (Birkenbihl et al., 2017). Furthermore, DIMERIZATION PARTNER (DP)-E2F-LIKE 1 (DEL1) directly targets EDS5 under non-stressed conditions to repress SA production (Chandran et al., 2014).

Due to their role as master regulators of SA production, SARD1 and CBP60g are themselves tightly regulated. Two transcription factors of the TGACG-binding factor (TGA) family, TGA1 and TGA4 are redundantly involved in the induction of SARD1 and CBP60g in Psm-triggered responses (Sun et al., 2018). SARD1 and CBP60g are negatively regulated by three proteins of the Calmodulin-binding transcription factor (CAMTA) family, CAMTA1, CAMTA2, and CAMTA3 (Kim et al., 2013; Sun et al., 2019; Kim et al., 2020). CAMTA3 directly binds to the promoter of $C B P 60 \mathrm{~g}$ for repression, whereas the negative effect of CAMTA transcription factors on SARD1 is still to be mechanistically shown (Sun et al., 2019). In non-triggered conditions, WRKY70 binds to the SARD1 promoter as means of repression of the SA defence (Zhou et al., 2018).

\subsubsection{SA perception}

After pathogen-triggered SA production defence outputs are generated by massive transcriptional reprogramming. SA reception is still a topic extensively investigated with many open questions. NON-EXPRESSOR OF PATHOGENESIS-RELATED GENES 1 (NPR1), NPR1-LIKE PROTEIN 3 (NPR3) and NPR4 are to date discussed as the main players in SA defence responses, but many more SA binding proteins exist, highlighting the fact that our current knowledge of SA perception might be restricted (Pokotylo et al., 2019). 
In 1997, NPR1 was found to be the main signalling hub for downstream transcriptional reprogramming after SA accumulation (Cao et al., 1997). NPR1 has been shown to bind SA, however, the consequences of the assumed conformational alteration are not known $(\mathrm{Wu}$ et al., 2012; Ding et al., 2018). NPR1 is thought to be mainly present in oligomeric form in the cytosol in unstressed conditions, stabilised by disulphide bonds (Tada et al., 2008). SA accumulation leads to redox state changes in the cell and THIOREDOXIN H3 (TRX-h3) and TRX-h5 catalyse NPR1 monomerisation (Mou et al., 2003; Tada et al., 2008). Monomeric NPR1 then translocates to the nucleus where it interacts with TGAs to activate defence gene expression (Zhang et al., 1999).

Independently of NPR1, NPR3 and NPR4, which also bind SA, act as negative regulators of the SA defence response under basal conditions (Ding et al., 2018). For their repressive activity, NPR3 and NPR4 require TGAs, suggesting that they directly act as repressors on promoters of SA-responsive genes (Ding et al., 2018). Accumulation of SA abolishes the

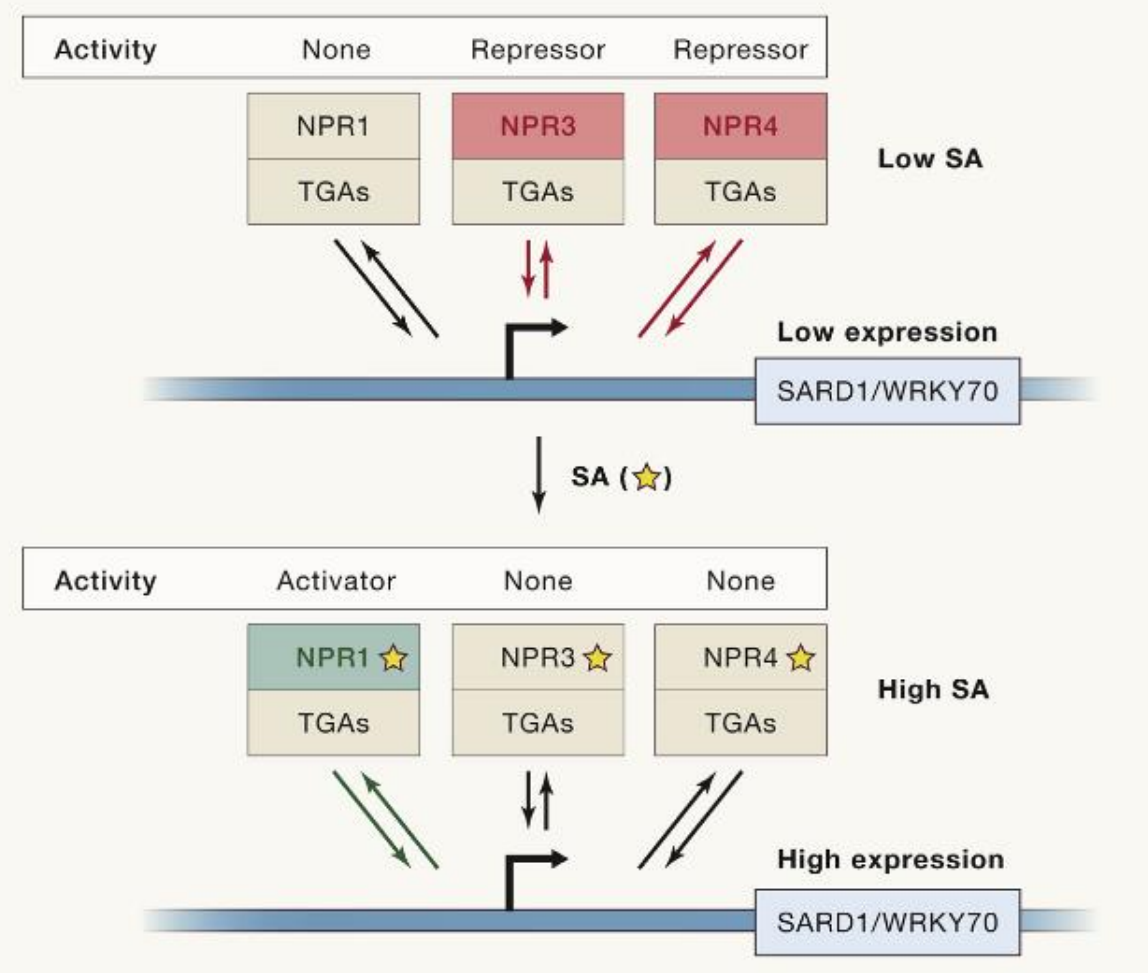

Figure 2. Current model of gene expression regulation in the SA defence response.

During low SA levels (upper panel) NPR3 and NPR4 repress promoters of SA-responsive genes via interaction with TGA factors. NPR1 cannot regulate gene expression in the absence of SA. SA accumulation (lower panel) activates NPR1 which induces defence gene expression. Binding of SA to NPR3 and NPR4 abolishes their repressor activity. Figure taken from Innes (2018). 
repressive effect of NPR3 and NPR4 on downstream gene expression (Ding et al., 2018). Figure 2 summarises the current model of transcriptional regulation of SA-responsive gene expression (Ding et al., 2018; Innes, 2018). In unstressed conditions NPR3 and NPR4 repress SA-responsive gene expression and NPR1 is inactive. Accumulation of SA simultaneously leads to defence gene induction by activating NPR1 and decreases repressive effects of NPR3 and NPR4.

\subsubsection{Systemic acquired resistance}

Despite inducing local defence gene expression, SA is also a crucial component of systemic acquired resistance (SAR). SAR describes a process in which local activation of immune responses by pathogen infection leads to defence priming of distal foliar tissues. If these systemic tissues then encounter pathogen attack, they mount defences more robustly. Plants unable to synthesise SA, as well as SA signalling mutants, show greatly attenuated SAR responses (Gaffney et al., 1993; Cao et al., 1997; Nawrath and Métraux, 1999; Bernsdorff et al., 2016). Nevertheless, grafting experiments of tobacco plants showed that SA, even though crucial for proper defence mounting in distal tissues, is not itself the mobile signal that travels to distal leaves to induce priming (Vernooij et al., 1994). Recent studies suggest that, instead, $\mathrm{N}$-hydroxypipecolic acid (NHP) is the mobile signal (Hartmann et al., 2018; Yildiz et al., 2021). Nevertheless, NHP and SA orchestrate SAR synergistically, with heavily intertwined gene regulation processes (Bernsdorff et al., 2016; Hartmann and Zeier, 2019; Yildiz et al., 2021).

\subsection{The JA/ET-mediated defence}

\subsubsection{JA-Ile biosynthesis}

In response to stimuli like wounding and pathogen attack JA-lle is rapidly synthesised. Figure 3 gives an overview of JA biosynthesis. In plastids, 18:3 and 16:3 fatty acids are converted into cis-12-oxo-phytodienoic acid (OPDA) and dinor-oxo-phytodienoic acid (dnOPDA) (Brash 
et al., 1988; Wasternack and Hause, 2013). This consecutively involves lipoxygenases (LOXs), ALLENE OXIDE SYNHASE (AOS) and ALLENE OXIDE CYCLASE (AOC). Subsequently, in peroxisomes, OPDA REDUCTASE 3 (OPR3) converts OPDA and dnOPDA to 3-oxo-2-(2'(Z)pentenyl)-cyclopentane-1-octanoic acid (OPC-8) and OPC-6, which are beta-oxidised to yield jasmonic acid (Breithaupt et al., 2001; Breithaupt et al., 2006; Wasternack and Hause, 2013). Jasmonic acid is exported from peroxisomes to the cytosol where JASMONOYL ISOLEUCINE CONJUGATE SYNTHASE 1 (JAR1) converts it into the bioactive form jasmonoyl-isoleucine (JA-lle) (Staswick and Tiryaki, 2004; Fonseca et al., 2009; Westfall et al., 2012). JA-Ile is translocated to the nucleus via JASMONATE TRANSPORTER 1 (JAT1) where its perception leads to massive transcriptional programming (Li et al., 2017). JA does not only play a major role in defence responses but is also required to coordinate developmental processes such as growth, fertility, senescence and responses to abiotic stress (Huang et al., 2017).

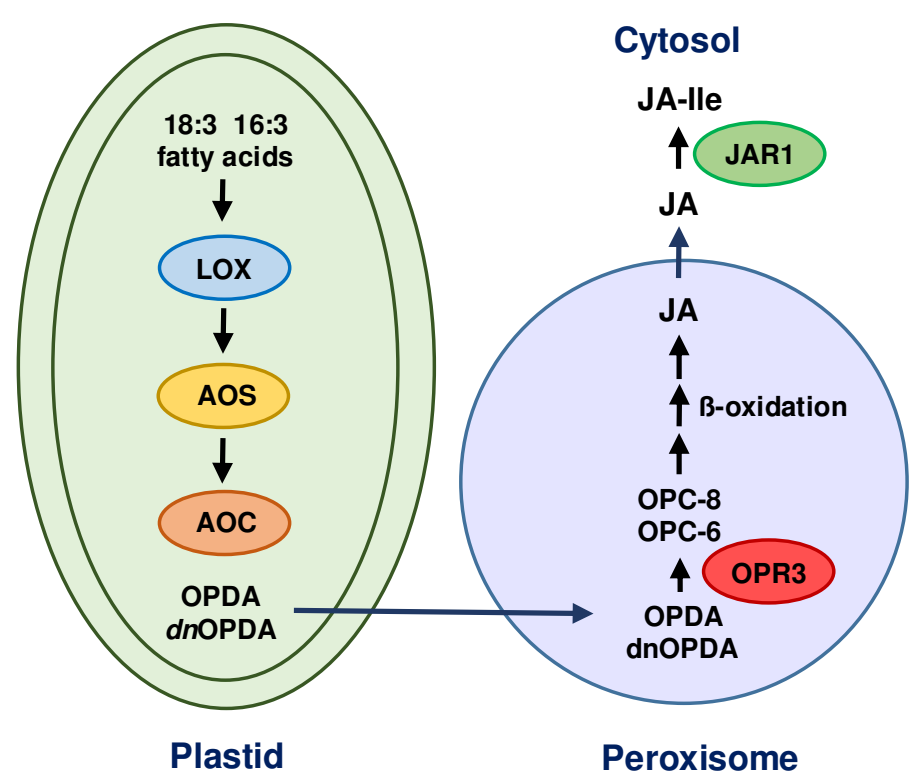

Figure 3. Biosynthesis of JA-lle in plastids, peroxisomes and the cytosol.

Tri unsaturated fatty acids are the starting point of synthesis and are converted to OPDA and InOPDA in plastids. In peroxisomes, OPDA and dnOPDA are converted to JA. The final step of conversion takes place in the cytosol where JA is modified to the bioactive form JA-lle. Modified from Dhakarey et al. (2016). 


\subsubsection{Perception of JA-lle by COl1 and JAZs}

JA-lle is perceived by CORONATINE INSENSITIVE 1 (COI1) together with co-receptors of the JASMONATE ZIM-domain (JAZ) family. JAZ proteins are repressors of transcription factors inducing JA-Ile-responsive genes, and their degradation upon JA-Ile perception leads to activation of the JA-defence pathway.

COI1 has two domains, a small N-terminal F-box domain and a large horseshoe shaped domain formed by 18 tandem LRRs (Sheard et al., 2010). The C-terminal LRR domain contains the surface binding pocket for JA-lle (Sheard et al., 2010). Additionally to JA-Ile binding, inositol pentakisphosphate is required for complex formation between COI1 and JAZs (Sheard et al., 2010). Through association with either ARABIDOPSIS SKP1-LIKE 1 (ASK1) or ASK2, CULLIN 1 (CUL1) and RING-BOX 1 (RBX1), COI1 forms a functional E3 ubiquitin ligase complex; SKP1/CULLIN/F-BOX (SCFCOI1) (Dai et al., 2002; Devoto et al., 2002; Xu et al., 2002). COI1 interacts with ASK1/2 via its F-box motif (Sheard et al., 2010). As a scaffolding protein, CUL1 links COl1 and ASK1/2 to RBX1, which binds the E2 ubiquitin-conjugating enzyme (Gray et al., 2002). Upon binding of JA-lle, COI1 and JAZ interaction is facilitated, leading to polyubiquitination of JAZ proteins and their degradation via the $26 \mathrm{~S}$ proteasome (Chini et al., 2007; Thines et al., 2007). Via its F-box motif, COl1 also interacts with MEDIATOR 25 (MED25), a protein of the multisubunit mediator complex crucial for transcriptional initiation, at JA-lle responsive promoters (An et al., 2017). The ability of JA-lle to promote the interaction of COI1 and JAZs is reduced in med25 mutants, suggesting that recruitment of COI1 to target promoters by MED25 is crucial for bringing together COI1 and JAZs (An et al., 2017). Activation of the JA pathway by perception of JA-lle is displayed in Figure 4.

The JAZ proteins are a family of 13 repressors. JAZs contain two important domains; the ZINCFINGER EXPRESSED IN INFLORESCENCE MERISTEM (ZIM) domain and the C-terminal JA-associated (Jas) domain. The Jas domain is important for JAZ degradation upon JA-lle sensing. It contains the highly conserved 20 amino-acid long Jas-degron consisting of an $\alpha$ helix for interaction with COI1 and a loop region important for trapping JA-Ile in its binding 


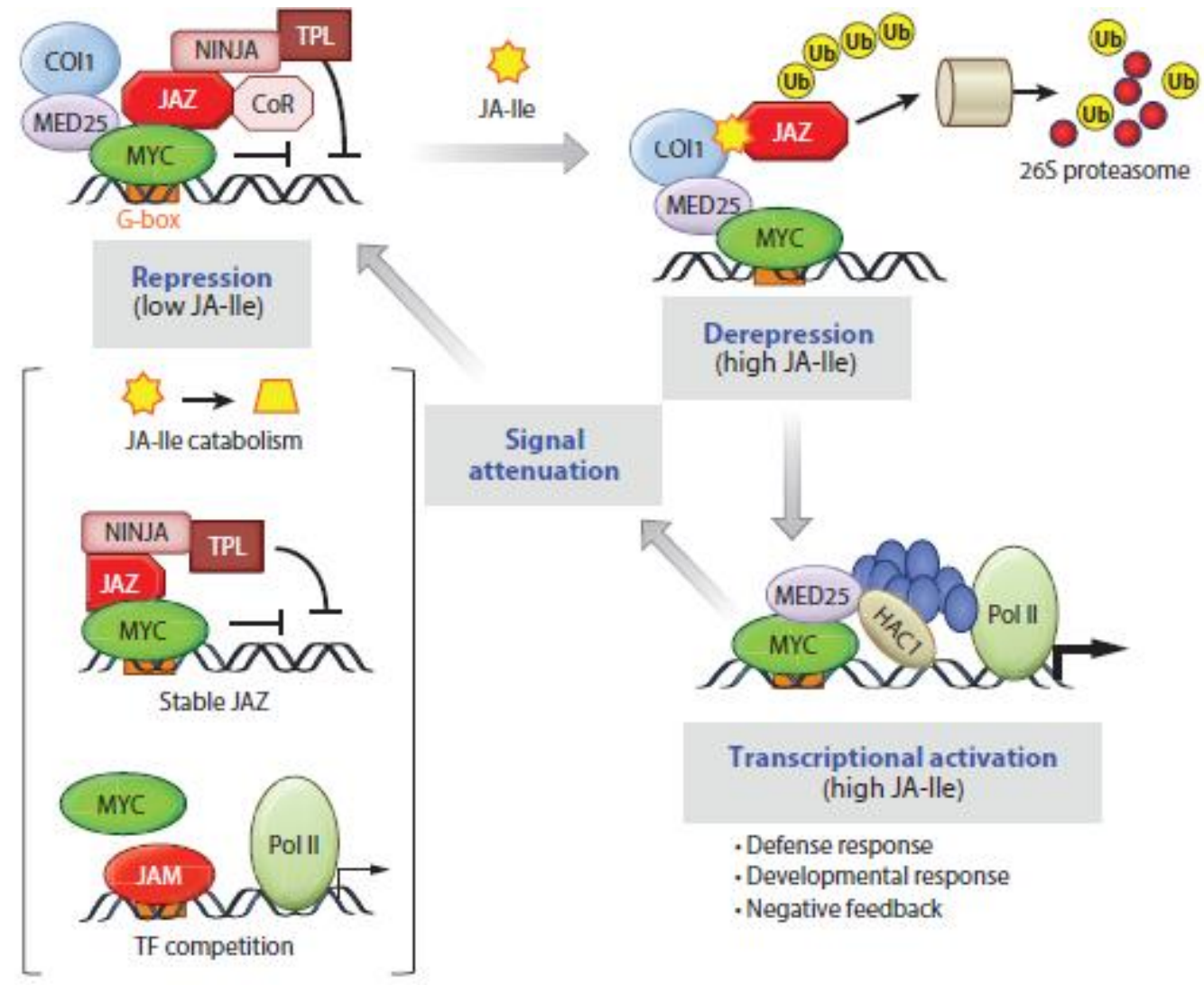

Figure 4. Induction of JA-signalling by JA-lle.

In basal conditions, transcription factors of JA-responsive genes, like MYCs, are repressed by JAZ proteins with their co-repressors TPLs and potentially other co-repressors (CoR). JA-lle facilitates binding of COI1 and JAZs which leads to JAZ polyubiquitination and degradation via the 26S proteasome. MYC and other transcription factors activate expression of target genes via the Polymerase II Transcription-Initiation Complex involving MED25 and HISTONE ACETYLTRANSFERASE OF THE CBP FAMILY 1 (HAC1). Activation of JA-lle catabolic genes, alternative spliced JAZs and negative competitors of MYCs reconstitutes negative feedback after JAdefence activation. Figure taken from Howe et al. (2018).

pocket (Sheard et al., 2010). Via their Jas domain JAZs also bind to and repress transcription factors of the JA response such as the basic helix-loop-helix (bHLH) transcription factors MYC2, 3 and 4 (Zhang et al., 2015).

Different mechanisms by which JAZs repress transcription factor activity have been demonstrated. In roots, JAZs recruit the adapter protein NOVEL INTERACTOR OF JAZ (NINJA) through their ZIM domain (Pauwels et al., 2010; Acosta et al., 2013). NINJA facilitates recruitment of the Groucho/Tup1-type co-repressor TOPLESS (TPL) and TPL-related proteins (Pauwels et al., 2010). Direct binding of TPL has also been observed for JAZ8 and is thought to occur with JAZ5, 6 and 7 as well (Kagale et al., 2010; Shyu et al., 2012). Moreover, JAZs 
have been shown to repress JA-signalling by blocking interaction of MYC3 and MED25, thereby hindering recruitment of the Polymerase II Transcription-Initiation Complex (Zhang et al., 2015; Zhang et al., 2017). JAZs have also been suggested to employ chromatin structure condensation for repression by interacting with histone modifying proteins HISTONE DEACETYLASE 6 (HDA6), LIKE HETEROCHOMATIN PROTEIN 1 (LHP1) and EMBRYONIC FLOWER 2 (EMF2) (Zhu et al., 2011; Li et al., 2021).

\subsubsection{JA defence branches}

JAZ proteins repress of a number of different transcription factors regulating various JA-Ilemediated plant processes (Song et al., 2011; Hu et al., 2013; Jiang et al., 2014). Two branches of JAZ-repressed JA-responses are specifically important in defence: the MYC branch and the ETHYENE RESPONSE FACTOR (ERF) branch.

Activation of the MYC2-, 3-, 4-regulated pathway leads to accumulation defence compounds such as glucosinolates and anthocyanins, launched in response to herbivores and wounding (Niu et al., 2011; Schweizer et al., 2013). A typical marker gene for activation of the MYCbranch is VEGETATIVE STORAGE PROTEIN 2 (VSP2).

The ERF branch of the JA-defence is co-regulated by JA and ethylene (ET) which are often induced simultaneously in response to necrotrophic pathogen attack (De Vos et al., 2007). The JA and ET pathway converge at the transcription factors ETHYLENE INSENSITIVE 3 (EIN3) and ETHYLENE INSENSITIVE 3-LIKE 1 (EIL1) which are repressed by JAZ proteins (Zhu et al., 2011). Downstream of their perception, both hormones synergistically activate EIN3/EIL1. Activation of EIN3/EIL1 in turn induces the transcription factor OCTADECANOIDRESPONSIVE ARABIDOPSIS AP2 59 (ORA59) that regulates downstream defences against necrotrophic pathogen attack (Pré et al., 2008). A marker gene for activation of the ERF-branch is PLANT DEFENSIN 1.2 (PDF1.2) (Penninckx et al., 1996; Zarei et al., 2011; Zhu et al., 2011). 


\subsubsection{Negative regulation of the JA defence}

The JA pathway is negatively regulated by the SA pathway, which will be discussed in 2.3 Crosstalk between the SA and the JA/ET defence pathway. Moreover, JA signalling is regulated via negative feedback loops. JA-lle-induced turnover of JAZ proteins induces genes involved in JA catabolism and negative regulators of JA signalling such as JA-associated MYC2-like (JAM) proteins and MYC2-Targeted BHLH (MTB) proteins that interfere with MYC activity (Koo et al., 2011; Nakata et al., 2013; Sasaki-Sekimoto et al., 2013; Song et al., 2013; Fonseca et al., 2014; Liu et al., 2019). JA-lle perception also leads to activation of JAZs themselves (Chung et al., 2010). Alternative splicing of these newly made JAZ transcripts can create JAZ proteins resistant to degradation via $\mathrm{SCF}^{\mathrm{CO} 11}$ desensitising the cell for JA-lle (Chung et al., 2010; Moreno et al., 2013; Zhang et al., 2017).

\subsection{Crosstalk between the SA and the JA/ET defence pathway}

The SA and JA pathway reciprocally antagonise each other. This is thought to occur to costeffectively launch the most efficient defence against the invading pathogen (Huot et al., 2014).

The JA pathway negatively regulates the SA pathway by both inhibiting SA biosynthesis and promoting inactivation of SA. Upon release from JAZ repression, MYC2 induces the transcription factors ANAC019, ANAC055 and ANAC072, which repress the ICS1 promoter (Zheng et al., 2012). Moreover, MYC2 induces BENZOIC ACID/SA CARBOXYL METHYLTRANSFERASE 1 (BSMT1) which converts SA to inactive methyl salicylate (MeSA) (Zheng et al., 2012).

The mechanisms by which the SA pathway negatively influences the JA pathway are less clear. The majority of SA-mediated crosstalk seems to happen downstream of JA biosynthesis (Leon-Reyes et al., 2010). Even though some biosynthetic genes of the JA pathway, such as AOS, AOC and OPR3, are reduced by SA treatment, PDF1.2 can still be repressed in the aos mutant, suggesting repressive effects downstream of JA biosynthesis (Leon-Reyes et al., 2010). Spoel et al. (2003) showed that NPR1 is required to repress JA-induced expression of 
PDF1.2 after SA treatment. This, however, does not require NPR1 in the nucleus so the mechanism of the repression is unclear (Spoel et al., 2003).

Moreover, SA-induced glutaredoxins (GRXs) have been shown to be able to repress PDF1.2 through suppression of ORA59, which requires TGA transcription factors (Ndamukong et al., 2007; Zander et al., 2012; Zander et al., 2014).

Li et al. (2004 and 2006) showed that SA-induced WRKY70 is involved in SA-JA crosstalk. Overexpression of WRKY70 has been shown to repress JA-induced PDF1.2 expression (Li et al., 2006). Nevertheless, SA is still able to repress PDF1.2 in wrky70 mutants, suggesting that WRKY70 might be competent but not necessary for SA-JA crosstalk or different WRKYs show redundancy for this role (Li et al., 2006).

\section{The vascular pathogen Verticillium longisporum}

\subsection{Infection cycle of $V$. longisporum}

The genus of the soil-borne ascomycete fungus Verticillium comprises ten species, several of which are plant pathogens (Inderbitzin, Davis, et al., 2011). Originally, V. Iongisporum was described as a subspecies of its close relative V. dahliae (Stark, 1961). However, in 1997, V. longisporum was recognised as a separate species (Karapapa et al., 1997). V. longisporum is unique within the Verticillium genus as it is the only allodiploid species among its haploid relatives (Ingram, 1968; Inderbitzin, Bostock, et al., 2011). For laboratory studies this makes $V$. longisporum harder to genetically manipulate and often the haploid $V$. dahliae is used to study plant-Verticillium interactions.

$V$. longisporum has a narrow host range, mostly restricted to Brassicaceae (Depotter et al., 2016). In particular, it is a threat to rapeseed (Brassica napus) production, especially in Europe (Depotter et al., 2016). In the field, an observable symptom of $V$. longisporum infection in $B$. napus is the appearance of dark unilateral stripes on the stems towards the end of the growing season (Depotter et al., 2016). A. thaliana is also a suitable host to study V. longisporum interactions with. Under laboratory conditions, $V$. longisporum causes similar symptoms in $B$. 
napus and $A$. thaliana, which include stunting, leaf chlorosis and premature senescence. Another observable symptom is vein clearing in infected foliage. Vein clearing is the result of trans-differentiation of chloroplast-rich bundle sheath cells into functional xylem vessels (Reusche et al., 2012).

$V$. longisporum is a vascular pathogen that enters hosts through the root and spreads through colonisation of the xylem. Figure 5 depicts the infection cycle of $V$. longisporum (Berlanger and Powelson, 2000). V. longisporum can produce melanised microsclerotia, thick-walled fungal cells that are long lasting resting structures (Stark, 1961). These can remain in the soil for many years (Depotter et al., 2016). Upon sensing root exudates of potential host plants these microsclerotia germinate and hyphae grow towards the host root (Berlanger and Powelson, 2000). Hyphae grow along root hairs towards the root surface, where the fungus penetrates preferentially lateral roots (Eynck et al., 2007). Before penetration, hyphae swell up and then form a thin penetration peg for breaching the plant cell wall (Eynck et al., 2007). Once hyphae have penetrated cell walls, they grow intercellularly and intracellularly towards the central cylinder and into the xylem (Eynck et al., 2007). In the xylem of B. napus, V. longisporum produces conidia that can be transported shootward by the transpiration stream (Depotter et al., 2016). Conidia can get trapped and germinate to colonise other xylem vessels throughout the plant (Depotter et al., 2016). Eynck et al. (2007) reported that V. longisporum never manages full colonisation of $B$. napus roots but remains within a few heavily colonised vessels. In the narrow roots of Arabidopsis, $V$. longisporum might grow in hyphal form though the root xylem to the shoot. At later stages of infection $V$. longisporum grows out of the xylem and starts feeding on the senescing plant matter (Eynck et al., 2007; Depotter et al., 2016). Therefore, $V$. longisporum is classed as a hemibiotrophic pathogen. At the vessel colonising phase $V$. longisporum employs a biotrophic lifestyle feeding from the relatively nutrient poor xylem sap, the later feeding of the foliage is the necrotrophic life stage (Depotter et al., 2016). During its necrotrophic phase $V$. longisporum produces microsclerotia that are released into the soil with the decomposed plant foliage (Heale and Karapapa, 1999). 


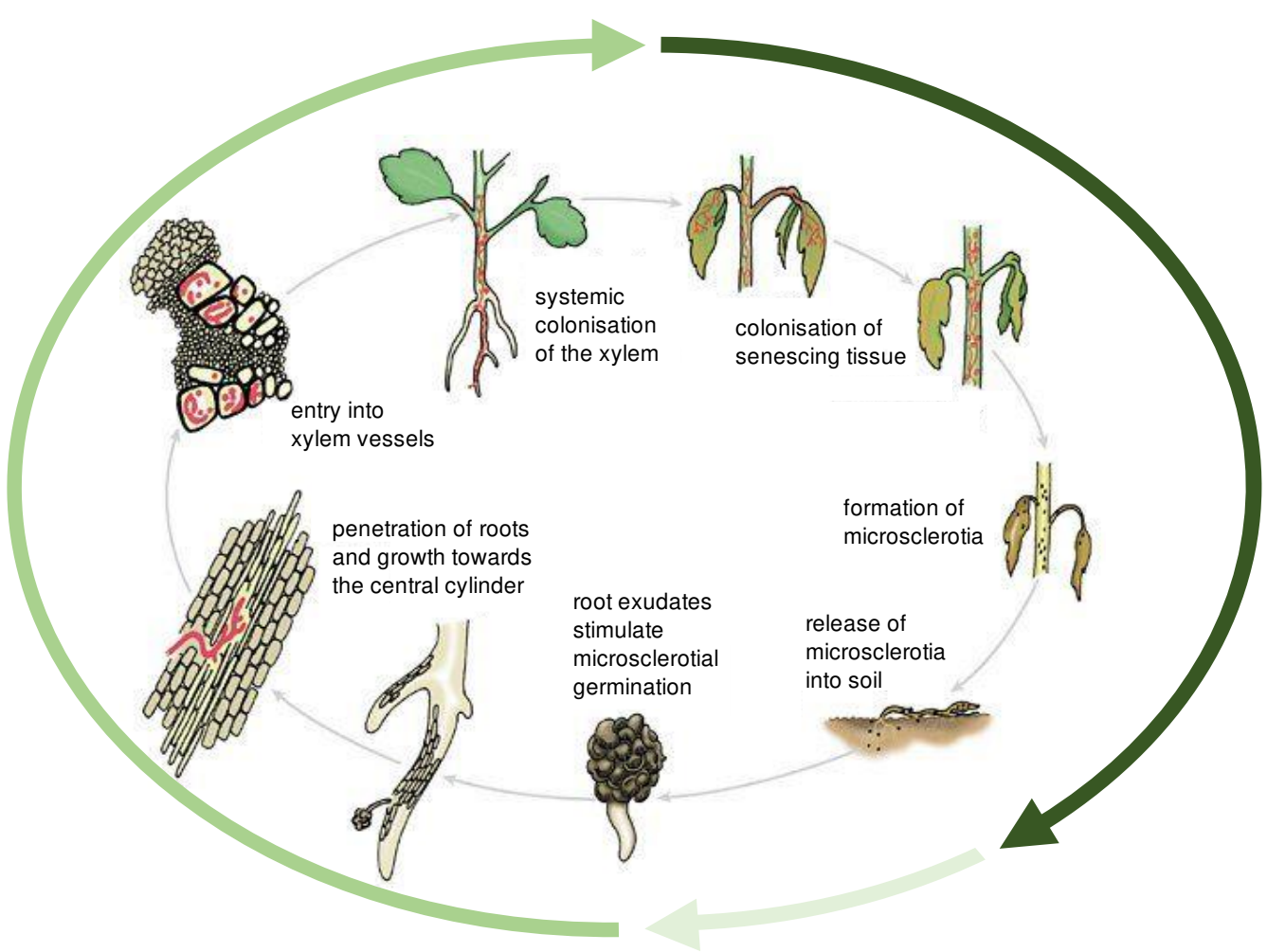

Figure 5. Infection cycle of $V$. longisporum.

Microsclerotia in the soil germinate upon sensing root exudates and penetrate host roots. Once hyphae have entered the root they grow towards the central cylinder and into the xylem. In the xylem, $V$. longisporum spreads though hyphal growth and production of conidia, reaching and colonising also upper parts of the host plant. During host senescence, $V$. longisporum leaves the xylem and starts feeding on plant material. Microsclerotia are formed and released in to the soil, where they rest until germination is stimulated. Pale green arrow: resting stage; light green arrow: biotrophic stage; dark green arrow: necrotrophic stage. Drawing by Vickie Brewster, coloured by Jesse Ewing. Figure modified from Berlanger and Powelson (2000).

\subsection{Disease control measures against $V$. longisporum are insufficient}

The vascular lifestyle of $V$. longisporum makes it inaccessible for fungicides during its residency in the plant. Hence, measures of reducing the primary inoculum in the soil have been concentrated on for disease control. The persistence of microsclerotia makes these measures difficult. As many fumigation techniques used to minimise the microsclerotia load in the soil have been banned for ecological reasons, crop rotations are one of the few effective measures to control infection events (Powelson and Carter, 1973; Depotter et al., 2016). The disease control of choice would be the use of resistant plants, however, to date these remain scarce. Moreover, monocultural farming promotes selection pressure for pathogens to overcome resistance, making the sustainability of this approach questionable (Lo Presti et al., 2015). 
Resistance genes have been identified for a few Verticillium species and the similar hemibiotrophic, soil-borne, vascular pathogen Fusarium oxysporum, not however, for $V$. longisporum.

In tomato, for example, $V e 1$ has been identified as a resistance gene against certain strains of V. dahliae, V. albo-atrum and F. oxysporum but not V. longisporum (Kawchuk et al., 2001; Fradin et al., 2009; De Jonge et al., 2012). Ve1 encodes a plasma membrane-localised RLP that detects Ave1, a fungal effector secreted during host colonisation (Kawchuk et al., 2001; Fradin et al., 2009; De Jonge et al., 2012).

Also, six RESISTANCE TO FUSARIUM OXYSPORUM (RFO1-6) genes have been identified against different races of F. oxysporum (Diener and Ausubel, 2005; Cole and Diener, 2013). Cole and Diener (2013) showed that induced expression of FMO3, which encodes an RLK, in roots upon infection hinders the spread of $F$. oxysporum in the vascular tissue.

Even though no resistance gene against $V$. longisporum has been identified, a few tolerant cultivars of $B$. napus exist. For example, the $B$. napus cultivar SEM 05-500256 produces higher constitutive and induced amounts of cell wall-bound and soluble phenolics as well as enhanced lignin deposition in roots and hypocotyl than susceptible cultivars (Eynck et al., 2009). These processes hinder the spread of $V$. longisporum to the shoot and convey tolerance (Eynck et al., 2009).

\subsection{Plant defences against $V$. longisporum}

Globally, rapeseed is the second largest oil seed crop, being important for production of oil for human nutrition, lubricants and biofuels (FAO Database, 2018). In 2017, rapeseed constituted $63 \%$ of all oil seed production in Europe, and demand is yet increasing (FAO Database, 2018). To improve the meagre disease management of $V$. longisporum infection on rapeseed, it is crucial to enhance our understanding of plant- $V$. longisporum interactions. 
A number of plant defences have been shown to be somewhat effective in combating infection by $V$. longisporum including barrier construction and production of antifungal compounds.

As described above, enhanced lignification to combat fungal spread can be effective (Eynck et al., 2009). In accordance, Fröschel et al. (2021) showed that V. longisporum targets endodermal barriers to get access into the central cylinder. Translating ribosome affinity purification (TRAP)-translatome analysis showed that genes involved in the formation of the endodermal barriers, casparian strip and suberin lamellae, were downregulated after infection with V. longisporum (Fröschel et al., 2021). Moreover, they claim that mutants impaired in proper casparian strip and suberin lamellae formation are more susceptible to $V$. longisporum (Fröschel et al., 2021). Hence, the structural barriers in differentiated plant roots constitute a basal defence to infection, that needs to be overcome by $V$. longisporum to reach the xylem.

The production of tryptophan-derived indole glucosinolates (IGs) against $V$. longisporum has been shown to be important in plant defence. Arabidopsis cyp79b2 cyp79b3 double mutants, carrying mutations in two CYTOCHROME P450 coding family members impaired in production of camalexin and IGs, showed enhanced susceptibility to $V$. longisporum infection (Iven et al., 2012). In accordance, Fröschel et al. (2019) showed that overexpression of different ERF transcription factors leads to decreased susceptibility against $V$. longisporum by induction of CYP81F2, another CYTOCHROME P450 family member involved in IG synthesis (Fröschel et al., 2019).

Analysis of apoplastic wash fluid from leaves of $A$. thaliana infected with $V$. longisporum at 25 dpi, showed enrichment of GERMIN-LIKE PROTEIN 3 (GLP3) (Floerl et al., 2012). Germins and Germin-like proteins (GLPs) are glycoproteins of the cupin superfamily, which have been shown to possess direct antifungal activities as well as defence signalling capabilities (Dunwell et al., 2008). Indeed, Germin-like-proteins from cotton (Gossypium hirsutum) have been shown to inhibit growth of V. dahliae and F. oxysporum (Pei et al., 2019; Pei et al., 2020). Overexpression of GLP1 from sugar beet (Beta vulgaris) in A. thaliana has been shown to 
reduce susceptibility to $V$. longisporum infection, by reducing fungal growth on the root surface and inside the root (Knecht et al., 2010).

The role of phytohormones in defences against $V$. longisporum is less clear. Ratzinger et al. (2009) reported accumulation of SA and SA-glucoside (SAG) in root and hypocotyl xylem sap as well as shoot extracts of $B$. napus at $14,21,28$ and 35 dpi with V. longisporum VI43. No differences in JA and abscisic acid (ABA) accumulation between mock-treated and infected plants at these time points were detected.

Zheng et al. (2019) showed that $B$. napus plants ectopically expressing the bacterial salicylate hydroxylase NahG, which degrades SA to catechol, accumulated higher fungal loads in the hypocotyl at 14 and 21 dpi with $V$. longisporum VI43. At 7 dpi, a resistant cultivar had higher levels of SA in the hypocotyl compared to a susceptible cultivar, however, no differences in SA levels were detected at 14 and 21 dpi anymore.

In contrast, Johansson et al. (2006) reported that the Arabidopsis JA and ET biosynthesis/signalling mutants jar1-1, coi1-16 and ein3-1 showed no difference in susceptibility to V. longisporum VD11 compared to wild-type (WT) plants. SA-biosynthesis deficient NahG and sid2-1 plants also showed no difference in susceptibility. In contrast, npr11 mutants were more susceptible to $V$. longisporum infection than WT plants. Moreover, SA treatment caused no phenotypic differences to infection, whereas pre-treatment with MeJA or the ethylene precursor 1-aminocyclopropane-1-carboxylic acid (ACC) increased tolerance to fungal infection. Similarly, Ralhan et al. (2012) showed no difference in susceptibility of JA and SA biosynthesis mutants aos and sid2-2, however, reported increased tolerance of coi1-t plants infected with $V$. longisporum VI43.

On the other hand, genes that promote susceptibility to $V$. longisporum have been found. Pröbsting et al. (2020) identified the susceptibility gene CALRETICULIN 1A (CRT1a) in B. napus and $A$. thaliana. CRT1a is induced after infection in $A$. thaliana and $B$. napus but when mutated, plants did not show severe loss of leaf area after infection anymore (Pröbsting et al., 2020). 


\section{Preliminary work}

Another susceptibility gene identified in $A$. thaliana for successful infection with $V$. longisporum is COI1 (Ralhan et al., 2012). After infection, the JA-Ile-receptor mutant coi1 shows greatly reduced infection symptoms compared to WT plants (Figure 6a) (Ralhan et al., 2012). The JA biosynthesis mutant aos shows WT-like symptoms (Figure 6a), hence, the tolerance of coi1 plants is not due to a disruption in the JA signalling pathway. Initial fungal colonisation of coi1 roots is not compromised, and the progression of infection is unaltered in coi1 plants compared to aos and WT until about 10 days past inoculation (dpi) (Ralhan et al., 2012). However, at later stages of infection (15-19 dpi) lower amounts of fungal biomass are found in shoots of coi1 as compared to WT and aos (Ralhan et al., 2012).

Some biotrophic pathogens like Pseudomonas syringae pv tomato (Pst) DC3000 produce coronatine which interacts with COI1 and activates the JA pathway to supress SA defences (Kloek et al., 2001). coi1 mutants, which are unable to perceive coronatine, are more tolerant to infection as they do not experience a suppression of the SA pathway (Kloek et al., 2001).

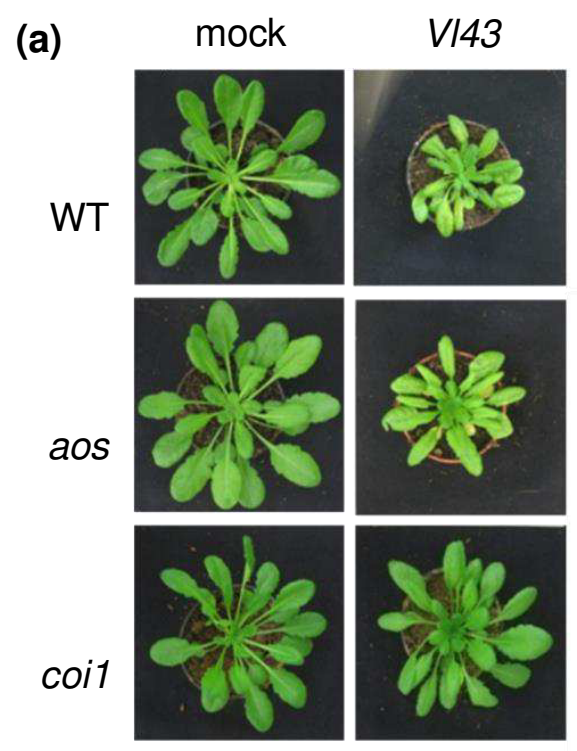

(b)
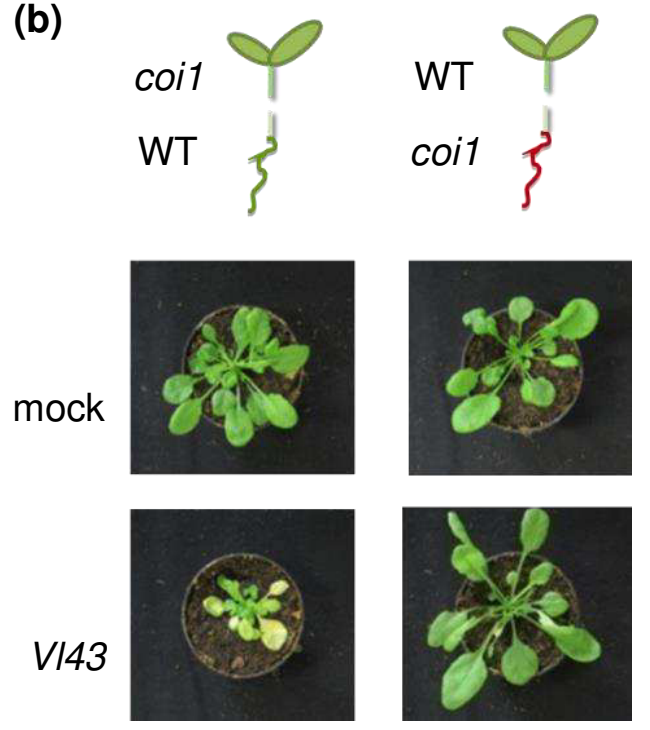

Figure 6. coi1 plants are tolerant against infection with $V$. longisporum.

(a) Disease symptoms in WT (Col-0), aos and coi1 shoots 15 days after mock treatment or inoculation with V. longisporum VI43. (b) Reciprocal grafts between WT (Col-0) and coi1 plants 21 days after mock treatment or inoculation with V. longisporum VI43. Figures modified from Ralhan et al. (2012). 
In the interaction with $V$. longisporum, the possibility of a fungal-derived JA-lle mimic activating COI1-mediated signal transduction in aos plants but not coi1 plants, was excluded by the observation that marker genes for neither the JA nor the JA/ET pathway were induced in aos plants after infection (Ralhan et al., 2012). In line with this, coi1 plants do not show high levels of PATHOGENESIS-RELATED PROTEIN 1 (PR1) after infection, which would hint at a hyperactivation of the SA pathway (Ralhan et al., 2012).

In grafting experiments, reciprocal grafts between Col-0 and coi1 plants were created (Ralhan et al., 2012). Infection of these chimeric plants showed that only plants which had coi1 roots would stay tolerant to infection (Figure 6b) (Ralhan et al., 2012).

To investigate gene expression patterns that could explain the observed tolerance mediated by coi1 roots, two RNA-Sequencing (RNA-seq) experiments were performed by a previous PhD student, Johanna Schmitz. The first RNA-seq data set was generated from axenically grown coi1, aos and WT roots at $4 \mathrm{dpi}$. The transcriptome data revealed basal de-repression of defence related genes specifically in mock-treated coi1 roots, however, no notable responses to fungal infection were observed on transcriptome level in any genotype. Therefore, to gain insight into the role of COI1 after infection with $V$. longisporum, a second RNA-seq analysis was performed in a more natural soil-based infection system at $10 \mathrm{dpi}$. Here, besides coi1, aos and WT roots, additionally the SA biosynthesis-impaired sid2 mutant was included to assess the contributions of both the JA and the SA defence to the root response triggered by $V$. longisporum. Again, mock-treated coi1 roots showed constitutive de-repression of defence-related genes. In contrast to the first RNA-seq data set, this time pronounced changes in the root transcriptomes were detectable after infection. 


\section{Thesis aims}

The de-repression of a large number of genes in mock-treated coi1 roots showed that COI1 has a role in gene repression. As the aos mutant shows WT-like expression of those genes, the new COl1 repressor function must be JA-lle-independent. The first aim of this thesis was to investigate if the novel COI1 repressor function works independently of components of the canonical JA-signalling pathway. Findings regarding this objective are described in Article 1. In the second RNA-seq dataset from the soil-based infection system, clear responses to the fungus were seen in root transcriptomes. The second aim of this thesis was to explore the role of COl1 in gene expression regulation upon infection with $V$. longisporum. Furthermore, potential reasons for the tolerance of coil plants were addressed. Article 2 presents the findings achieved in understanding these processes. 


\title{
III. Article 1
}

\section{The jasmonoyl-isoleucine receptor CORONATINE INSENSITIVE1} suppresses defense gene expression in Arabidopsis roots independently of its ligand

Louisa Ulrich ${ }^{1}$, Johanna Schmitz ${ }^{1}$, Corinna Thurow and Christiane Gatz ${ }^{2}$

Department of Plant Molecular Biology and Physiology, Albrecht-von-Haller Institute for Plant Sciences, University of Göttingen, Julia-Lermontowa-Weg 3, 37077 Göttingen, Germany

\author{
${ }^{1}$ Authors contributed equally to this work \\ ${ }^{2}$ Corresponding author: cgatz@gwdg.de
}

This article was published online on 18/06/21 in The Plant Journal and can be found at https://doi.org/10.1111/tpj.15372

\section{Detailed contributions by the PhD candidate:}

Louisa Ulrich prepared, performed and analysed the following experiments: gene expression analyses displayed in Figure 3a, Figure 5a and 5c, Figure 6, Figure S4 and Figure S9; western blot analyses displayed in Figure $5 \mathrm{~b}$ and Figure S12; yeast two hybrid analysis displayed in Figure S11 (right panel); the transient reporter assay displayed in Figure S12 and documentation of seed pod production displayed in Figure S13. Louisa Ulrich prepared clones

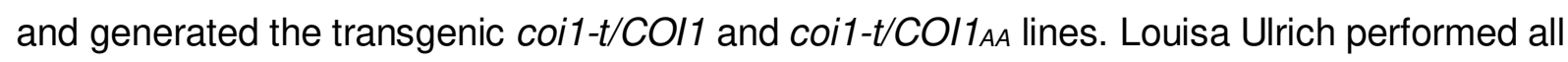
statistical analysis for the article with exception of the RNA-seq data analysis. Louisa Ulrich created all Figures with the exception of Figure 1a, Figure 2a, Figure S1 and Figure S10. 


\section{Graphical Abstract}

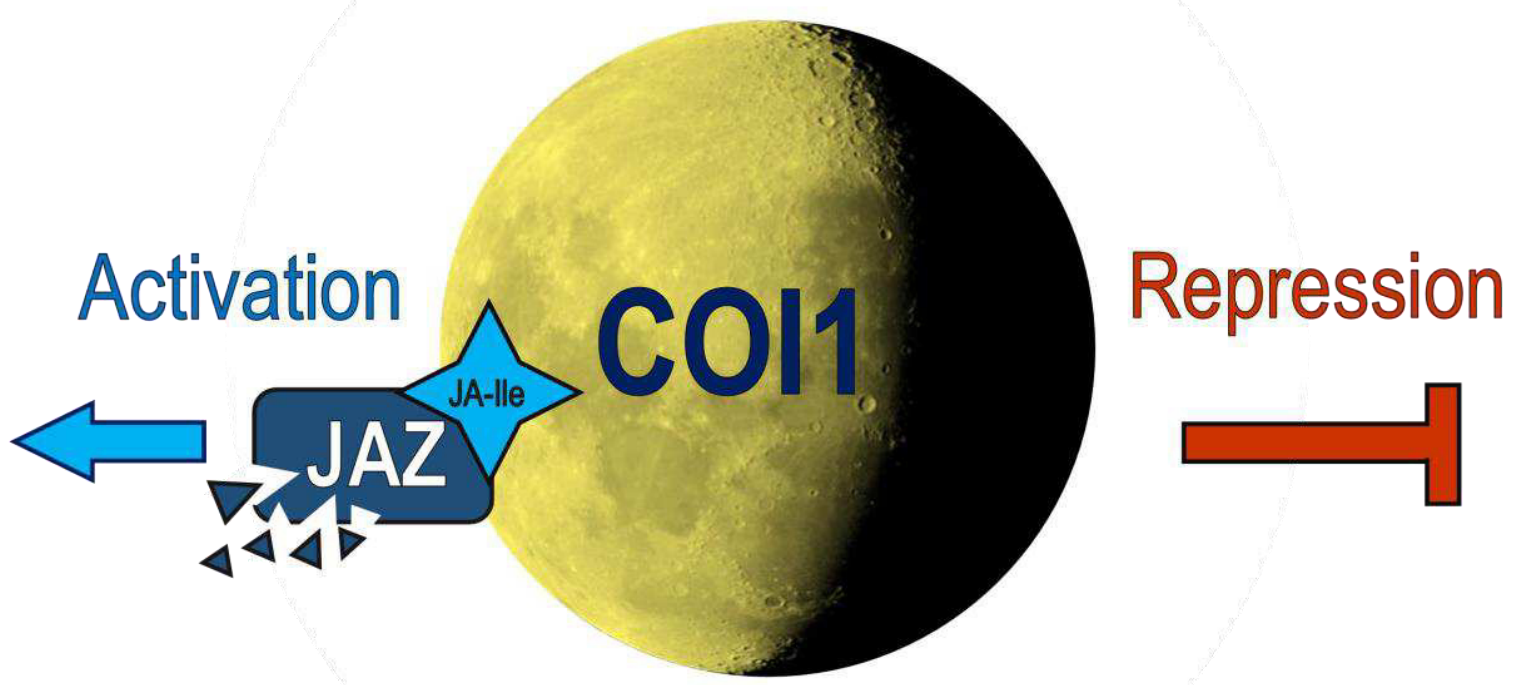

\section{Significance Statement}

Phenotypic differences of hormone receptor and corresponding hormone biosynthesis mutants are unexpected. Such an unusual scenario was discovered for COI1 which affects the root transcriptome even when disconnected from its signaling pathway. 


\title{
The jasmonoyl-isoleucine receptor CORONATINE INSENSITIVE1 suppresses defense gene expression in Arabidopsis roots independently of its ligand
}

\author{
Louisa Ulrich ${ }^{\dagger}$, Johanna Schmitz ${ }^{\dagger}$, Corinna Thurow and Christiane Gatz* \\ Department of Plant Molecular Biology and Physiology, Albrecht-von-Haller-Institute for Plant Sciences, University of \\ Göttingen, Göttingen, Germany
}

Received 7 February 2021; accepted 2 June 2021.

*For correspondence (e-mail cgatz@gwdg.de).

These authors contributed equally to this work

\begin{abstract}
SUMMARY
The F-box protein CORONANTINE INSENSITIVE1 (COI1) serves as the receptor for the plant hormone jasmonoyl-isoleucine (JA-lle). COI1, its co-receptors of the JASMONATE ZIM-domain (JAZ) protein family, and JA-Ile form a functional unit that regulates growth or defense mechanisms in response to various stress cues. Strikingly, COI1, but not JA-Ile, is required for susceptibility of Arabidopsis thaliana towards the soilborne vascular pathogen Verticillium longisporum. In order to obtain marker genes for further analysis of this JA-Ile-independent COI1 function, transcriptome analysis of roots of coi1 and allene oxide synthase (aos) plants (impaired in JA biosynthesis) was performed. Intriguingly, nearly all of the genes that are differentially expressed in coi1 versus aos and wild type are constitutively more highly expressed in coi1. To support our notion that COI1 acts independently of its known downstream signaling components, coi1 plants were complemented with a COI1 variant $\left(\mathrm{COI1}_{\mathrm{AA}}\right)$ that is compromised in its interaction with JAZs. As expected, these plants showed only weak induction of the expression of the JA-lle marker gene VEGETATIVE STORAGE PROTEIN2 after wounding and remained sterile. On the other hand, genes affected by COI1 but not by JA-lle were still strongly repressed by $\mathrm{COI1}_{\mathrm{AA}}$. We suggest that COI1 has a potential moonlighting function that serves to repress gene expression in a JA-Ile- and JAZ-independent manner.
\end{abstract}

Keywords: CORONATINE INSENSITIVE1, JASMONATE ZIM-domain, jasmonoyl-isoleucine, moonlighting, repression, root.

\section{INTRODUCTION}

Hormones serve as signaling molecules that are crucial for the regulation of development, growth, and anti-stress programs. It is generally accepted that internal or external cues lead to increased cellular hormone concentrations. Binding of hormones to their cognate receptors is crucial for the activation of signaling cascades resulting in cellular responses like transcriptional re-programming. Consistent with this concept, hormone receptor mutants usually have similar phenotypes as the corresponding hormone biosynthesis mutants.

The jasmonoyl-isoleucine (JA-lle) receptor CORONATINE INSENSITIVE1 (COI1) acts as an adaptor protein within the E3 ubiquitin ligase complex $\mathrm{SCF}^{\mathrm{CO} 11}$ and forms - upon hormone binding - a transient ternary complex with JASMONATE ZIM-domain (JAZ) proteins, resulting in their ubiquitination and subsequent degradation through the
26S proteasome (Chini et al., 2007; Thines et al., 2007) JAZs interfere with the activity of various transcription factors, including MYC2, MYC3, MYC4 (Fernandez-Calvo et al., 2011), ETHYLENE INSENSITIVE3 (EIN3), and EIN3 LIKE1 (EIL1) (Zhu et al., 2011). Reduced JAZ protein levels thus lead to the activation of promoters controlled by these factors. In Arabidopsis thaliana, the pathway is initiated during stamen development (Jewell and Browse, 2016) and after various stress cues like wounding and insect feeding (McConn et al., 1997), pathogen infection (Vijayan et al., 1998), and salt treatment (Geng et al., 2013). Al these processes are impaired in both the coi1 mutant and the JA-lle biosynthesis mutant allene oxide synthase (aos).

However, coil and aos do not always show the same phenotype. For instance, root growth of the JA biosynthesis mutant aos was as sensitive to $4 \mu \mathrm{m}$ of the ethylene (ET) precursor 1-aminocyclopropane-1-carboxylic acid (Adams and Turner, 2010) or phytoprostane PPA1 (Stotz et al., 2013) 


\section{Louisa Ulrich et al.}

as the wild type, while the coi1-16 mutant was less affected. More recently, COI1, but not JA-lle, was shown to be required for the extracellular adenosine $5^{\prime}$-triphosphate (eATP)-mediated reinforcement of plant defense against the necrotrophic fungus Botrytis cinerea. Here, eATP treatment of aos plants led to the degradation of a transgenic JAZ1: GUS fusion protein (Tripathi et al., 2018).

An unexpected difference between coi1 and aos plants has also been observed after infection with the soil-borne vascular pathogens Fusarium oxysporum (Thatcher et al., 2009) and Verticillium longisporum (Ralhan et al., 2012). In both pathosystems, coi1 was more tolerant than wild type and aos. It was hypothesized that $F$. oxysporum or $V$. longisporum might synthesize JA-lle or a JA-lle mimic to induce susceptibility through COI1, a strategy that has been demonstrated for the coronatine-producing bacterial pathogen Pseudomonas syringae pv tomato (Pst) DC3000 (Kloek et al., 2001). Like JA-lle, coronatine interacts with COI1, which results in the degradation of JAZ repressor proteins (Chini et al., 2007; Thines et al., 2007). Activation of the JA pathway antagonizes SA-dependent defense responses, which explains the observed higher resistance of coi1 (Brooks et al., 2005). Hence, infection of aos plants with coronatine-producing Pseudomonas syringae pv maculicola (Psm) ES4326 leads to the induction of the expression of JA-responsive genes (Wang et al., 2008). In contrast, after $V$. longisporum infection, expression of neither the JA marker gene VEGETATIVE STORAGE PROTEIN2 (VSP2) nor the JA/ET marker gene PLANT DEFENSIN1.2 (PDF1.2) was induced in the aos mutant. This indicates that fungal compounds that would activate the known COI1-dependent signal transduction chain are not produced by V. longisporum (Ralhan et al., 2012). Consistently, the tolerance observed in coi1 is not associated with hyper-activation of the SA-induced gene PATHOGENESISRELATED1 (PR1) (Ralhan et al., 2012), which was observed after infection with Pst DC3000 (Kloek et al., 2001). Together, our results have unraveled a COI1 activity which acts independently from JA-Ile or any JA-Ile mimic (Ralhan et al., 2012). Grafting experiments between coi1 rootstocks and wild-type scions (and vice versa) revealed that the JAlle-independent COl1-mediated susceptibility towards $F$. oxysporum and $V$. longisporum requires the wild-type COI1 allele in roots (Ralhan et al., 2012; Thatcher et al., 2009).

Here, we approached the question whether known components of the JA-lle signaling pathway are required for the JA-Ile-independent COI1 function. Since coi1-mediated tolerance is a complex phenotype, we aimed to find a simpler proxy for our analysis. Therefore, we performed transcriptome analysis of roots of coi1, aos, and wild-type plants. We found that the transcriptome of coi1 roots is characterized by a set of constitutively expressed genes. Using selected marker genes, we show that COI1 can function as a repressor even when the interaction between COI1 and JAZ proteins is severely impaired. Moreover, other known components of the COI1 signaling cascade, like JAZ-regulated transcription factors MYC2, MYC3, and MYC4 or EIN3 and EIL1, do not contribute to COI1mediated repression of the marker genes. It is concluded that either COI1 facilitates degradation of yet unknown substrates or, alternatively, it is a moonlighting protein.

\section{RESULTS}

\section{Segregating plants from heterozygous $\mathrm{COI} /$ coi1 and AOS/aos populations were used for RNA-seq analysis}

Previous grafting experiments have shown that the coi1 allele has to be present in roots to confer tolerance against either F. oxysporum or V. longisporum (Ralhan et al., 2012; Thatcher et al., 2009). Therefore, the transcriptomes of roots from $V$. longisporum-infected and uninfected wildtype, coi1-t, and aos plants were analyzed. Since defects in JA perception or synthesis lead to male sterility (von Malek et al., 2002; Park et al., 2002; Xie et al., 1998), plants with strong coi1 alleles can only be maintained as a heterozygous population. In contrast, the aos phenotype is rescued by methyl jasmonate (MeJA) treatment during flower development. To avoid differences in the history of the seed batches, we generated heterozygous AOS/aos plants by back-crossing the homozygous aos mutant with wildtype Col-0. Individual plantlets of the segregating AOS/aos and COI1/coi1-t (Mosblech et al., 2011) populations were infected with $V$. longisporum. After genotyping, RNA was extracted from 30 to 33 roots per segregating wild type and homozygous coil or aos mutants (mock and infected) and replicates from three independent experiments were used to construct libraries for Illumina sequencing.

\section{COI1 suppresses gene expression in the absence of jasmonoyl-isoleucine}

To obtain a first impression of the global structure of the transcriptome dataset, principal component analysis was performed (Figure 1a). Surprisingly, clusters representing mock-treated versus infected plants of one genotype showed overlapping datasets. This result indicates that the plantlets did not strongly respond to the fungal infection under our conditions. In contrast, the datasets of the four genotypes showed a clear separation, with those representing the transcriptomes of the two segregating wild types $\left(\mathrm{WT}_{\text {coi1-t }}\right.$ and $\mathrm{WT}_{\text {aos }}$ ) being most related, though still distinct. The transcriptome of the aos mutant was more related to that of its segregating $\mathrm{WT}_{\text {aos }}$ than the transcriptome of the coi1-t mutant to its segregating $\mathrm{WT}_{\text {coi1-t }}$. Moreover, the coi1-t transcriptome was clearly different from the aos transcriptome.

Since our main aim was to explore the JA-lleindependent function of COI1, we focused on those genes 
(a)

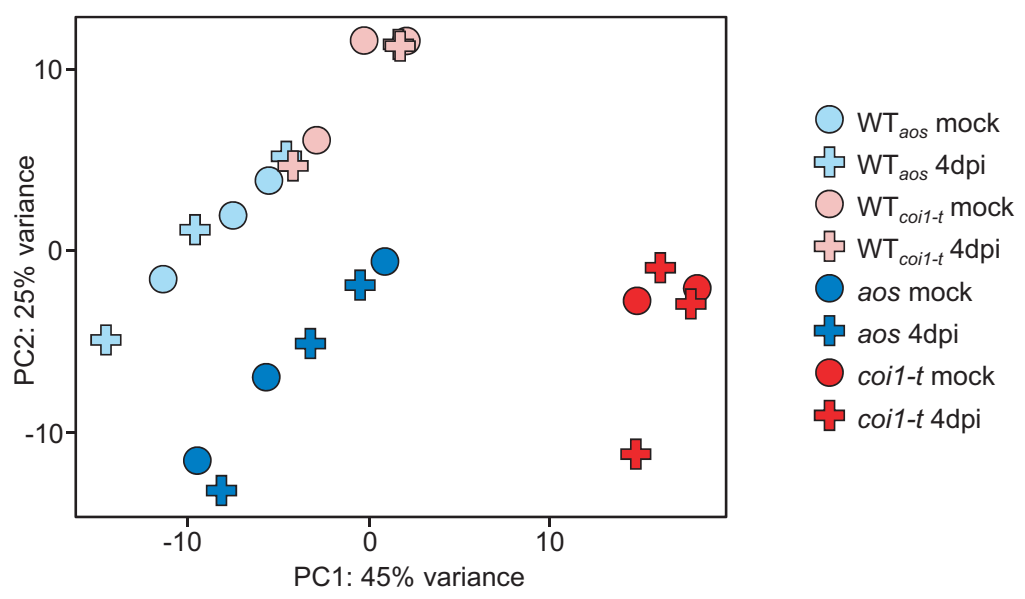

(b)
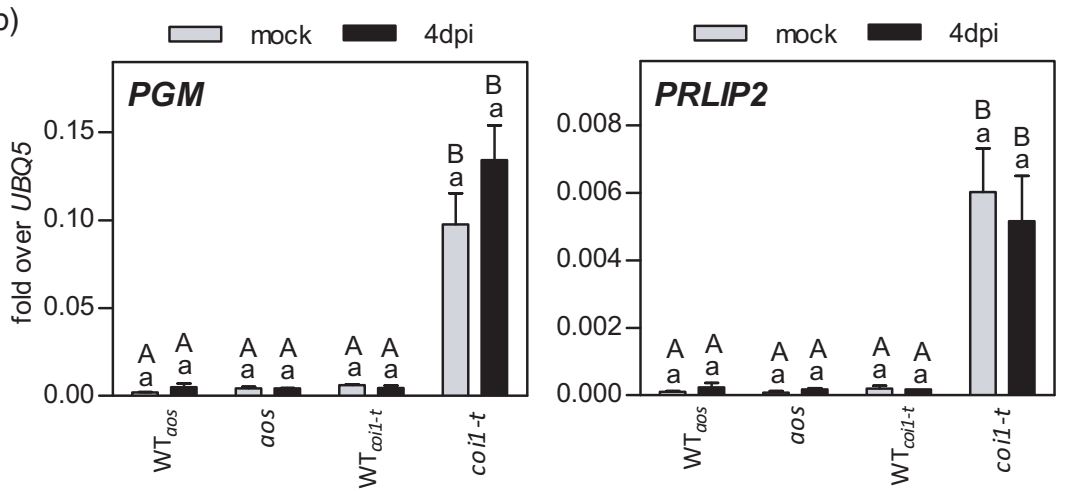

Figure 1. The coi1-t transcriptome differs from the transcriptomes of aos and the respective segregating wild types.

(a) Principal component analysis of the normalized transcriptome data obtained from RNA-seq analysis. Symbols represent biological replicates resulting from three independent experiments. Note that only two mock samples (coi1-1) were processed. Wild-type $\left(\mathrm{WT}_{\text {aos }}\right.$ and $\mathrm{WT}_{\text {coi1-t }}$ ) samples originated from the segregating offspring of the heterozygous aos and coi1-t populations.

(b) PHOSPHOGLYCERATE MUTASE (PGM) and PATHOGENESIS-RELATED LIPASE 2 (PRLIP2) expression, measured by qRT-PCR. The same RNA samples as in (a) were used. For statistical analysis, two-way ANOvA was performed followed by Bonferroni's multiple comparison test; lowercase letters denote significant differences within each genotype between mock and 4 days post-infection $(P<0.05)$, uppercase letters denote significant differences between genotypes subjected to the same treatment $(P<0.05)$.

that were differentially ( $>$ twofold; $P<0.05$ ) expressed in coi1-t as compared to both wild types and the aos mutant (Table S1 and sub-tables). In the mock-treated samples, only 12 genes were more lowly expressed in coi1-t than in the other genotypes, while 222 genes were more highly expressed. Analysis of the infected samples yielded the same pattern, with only nine genes being expressed at lower levels and 199 being de-repressed in coi1-t. In infected and mock-treated coi1-t plants, 167 genes were more highly expressed, indicating that increased expression of this set of genes is robust (Figure S1). Only two genes were expressed at lower levels in coi1-t irrespective of the treatment.

Figure $1 \mathrm{~b}$ displays the expression patterns of two representative genes from the group of 167 genes that were highly de-repressed in coi1-t. Quantitative reverse transcription PCR (qRT-PCR) analysis of the material subjected to RNA sequencing (RNA-seq) analysis indicated that these genes (AT3G60415 [PHOSPHOGLYCERATE MUTASE \{PGM\}] and AT5G24200 [PATHOGENESIS-RELATED LIPASE2 \{PRLIP2 \}]) (Jakab et al., 2003) were about 50-fold more highly expressed in coi1-t than in the two wild-type lines and the aos mutant (Figure 1b). In contrast, primary target genes of the canonical COI1-dependent pathway ( $J A Z 1, J A Z 9$, and $J A Z 10)$ are expressed at lower levels in both coi1-t and aos (Figure S2). As expected, the expression pattern was independent of whether plants were mock-treated or infected. Furthermore, increased expression of PGM and PRLIP2 was confirmed in coi1-1 (Xie et al., 1998) and the temperature-sensitive coi1-16 mutant (Ellis and Turner, 2002) (Figure S3). Higher transcript levels of $P G M$ and PRLIP2 were also observed in uninfected roots of soil-grown plants (Figure S4a). Under these conditions, one of the two genes with lower expression levels in roots 
of axenically grown seedlings was not affected by coi1 (AT2G05420) (Figure S4b). For the other (AT5G54450), expression was so low that no specific PCR product was detected. Hence, these genes are unlikely to act as repressors of the large number of de-repressed genes in coi1. These analyses indicate that COI1 can interfere with the expression of specific genes when acting independently of JA-Ile.

Functional enrichment analysis of the 167 genes with elevated expression levels in coi1-t demonstrated that Gene Ontology (GO) terms associated with immune responses were more than fivefold enriched (Figure 2a). In particular, processes connected to the defense hormone salicylic acid (SA) were overrepresented. The expression of the SA biosynthesis gene ISOCHORISMATE SYNTHASE1 (ICS1) (Wildermuth et al., 2001) was 2.8-fold higher in coi1-t than in aos (Figure 2b). Enhanced expression of ICS1 was also observed in coi1-1, but not in coi116 (Figure S3).

Next, we analyzed whether elevated expression of ICS1 was the primary reason for the increased transcript levels of SA-related genes. To this end, we crossed coi1-1 and the SA biosynthesis mutant sid2-2. Analysis of the resulting coi1-1 sid2-2 double mutant showed that enhanced expression of PGM and PRLIP2 in coi1-1 occurred in the absence of ICS1-derived SA (Figure 3a; Figure S5). This correlates with the tolerance phenotype after infection with $V$. longisporum which was observed in coi1-1 and coi1-1 sid2-2 but not in wild type, aos, and sid2-2 (Figure 3b).

We have shown previously that - similar to coi1-1 sid2-2 - the JA-lle-deficient coi1-t aos double mutant is as tolerant as coi1-t (Ralhan et al., 2013). Likewise, PGM and PRLIP2 expression was as high in coi1-t aos as in coi1-t, demonstrating that JA-lle does not induce the expression of these genes in the absence of its receptor (Figure S6).

\section{COI1-mediated repression is apparently independent of its interaction with JAZ proteins}

JA-lle-facilitated interaction of COI1 with JAZ repressor proteins leads to their degradation (Chini et al., 2007; Thines et al., 2007). In vitro, recombinant COI1 does not interact with the JAZ1 degron in the absence of the ligand (a)

$$
\begin{aligned}
& \text { Gene Ontology term (biological process) Percentage of genes }
\end{aligned}
$$

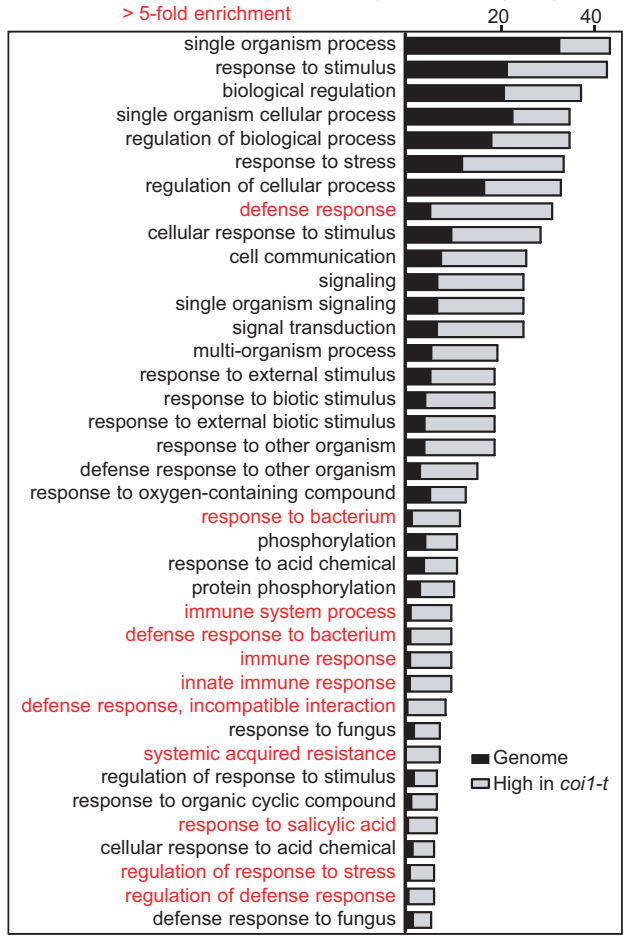

(b)

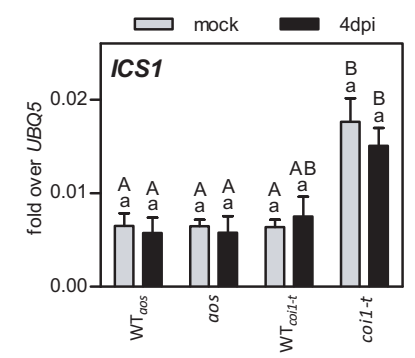

Figure 2. Genes related to salicylic acid-mediated immune responses are de-repressed in coi1-t roots.

(a) Gene Ontology (GO) overrepresentation analysis of 167 genes that were more highly expressed $(>$ twofold; $P<0.05$ ) in coi1-t as compared to aos and the respective segregating wild types. Black bars indicate the percentage of genes of each GO term found within the group of all annotated genes of the Arabidopsis genome. Gray bars indicate the percentage of genes of each GO term found within the group of 167 genes de-repressed in coi1-t.

(b) ISOCHORISMATE SYNTHASE 1 (ICS1) transcript levels, measured by qRT-PCR. The same RNA samples as for the RNA-seq experiment were used. For statistical analysis, two-way ANOVA was performed followed by Bonferroni's multiple comparison test; lowercase letters denote significant differences within each genotype between mock and 4 days post-infection $(P<0.05)$, uppercase letters denote significant differences between genotypes subjected to the same treatment $(P<0.05)$. WT $\mathrm{W}_{a s}$ and $\mathrm{WT}_{\text {coir-1 }}$ are the two wild-type lines obtained from the segregating offspring of heterozygous aos and coi1-t seeds. 
(a)

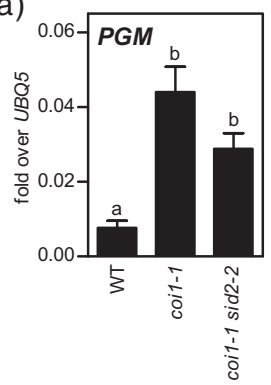

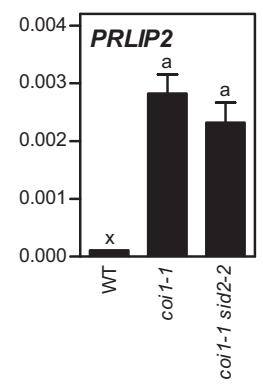

(b)

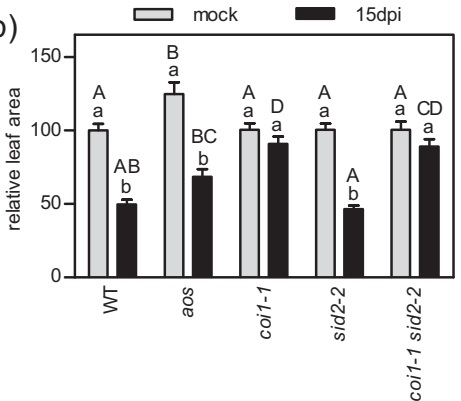

Figure 3. ICS1-derived SA is not responsible for de-repression of PGM and PRLIP2 or the tolerant disease phenotype of coi1-1. (a) PGM and PRLIP2 transcript levels, measured by qRT-PCR. RNA was extracted from roots of sand-soil-grown coi1-1, coi1-1 sid2-2, and Col-0 plants 10 days after mock treatment and subsequent transfer to soil. Bars show the mean \pm SEM of six roots per genotype. For statistical analysis, one-way ANovA was performed followed by Tukey's multiple comparison test; lowercase letters denote significant differences between samples $(P<0.05)$, $\mathrm{x}$ indicates that for expression of PRLIP2 in wild type only one value was obtained, while the other five fell below the detection threshold in our analysis. Thus, an unpaired two-tailed Student $t$-test was performed between coi1-1 and coi1-1 sid2-2 samples.

(b) Leaf area of mock-treated and V. longisporum-infected plants at 15 days post-infection (dpi). Plants were grown on sand-soil mixture and transferred to soil after treatment. Bars show the mean \pm SEM of 48 plants from three independent experiments. Values from mock-treated wild-type plants are set to 100 . For statistical analysis, two-way ANOVA was performed followed by Bonferroni's multiple comparison test; lowercase letters denote significant differences within each genotype between mock and $15 \mathrm{dpi}(P<0.05)$, uppercase letters denote significant differences between genotypes subjected to the same treatment $(P<0.05)$.

(Yan et al., 2018). Still, COI1 functions as a repressor of gene expression in roots of the aos mutant, suggesting that JAZ proteins are not involved in this process. Nevertheless, to explore potential ligand-independent degradation of specific JAZ proteins in vivo, we tested for COI1mediated activation of the JAZ1 promoter in transiently transformed protoplasts of the coi1-1 aos mutant. This experimental system allows to repress a $J A Z 1_{p}:$ luciferase reporter construct upon expression of specific JAZs as effector proteins. Upon additional expression of COI1 and incubation of protoplasts in the presence of coronatine, luciferase activity is induced (Li et al., 2019). In the absence of coronatine, none of the repressors were degraded as deduced from the absence of any positive effect of COI1 on luciferase activity (Figure S7). Thus, at least in protoplasts, no ligand-independent degradation of specific JAZ proteins by COI1 was observed.

To obtain further evidence for the JAZ-independent COI1 function, we analyzed transgenic plants constitutively expressing the non-degradable JAZ1 $\triangle 3 A-G U S$ fusion protein which mimic the coi1 phenotype in various aspects (male sterility, JA-insensitive root growth, severely compromised wound-induced expression of JA marker genes in leaves) (Thines et al., 2007). For reasons that have remained unknown, the coi1 phenotype was only partially mimicked with respect to reduced expression of JAZ10 in MeJA-treated roots (Figure S8). With this positive control not fully functioning, we did not further draw any conclusion from our result that PRLIP2 expression was not affected in 35S:JAZ1 $13 A$ :GUS plants.

Alternatively, we used the jaz decuple $(j a z D)$ mutant, which is defective in JAZ1-7, 9, 10, and 13, resulting in constitutive activation of both JA and ET responses (Guo et al., 2018). In this mutant, $P G M$ expression was not significantly reduced (Figure S9). Since PRLIP2 transcript levels are already low in wild-type roots grown in soil, we chose SYSTEMIC ACQUIRED RESISTANCE DEFICIENT1 (SARD1) as a second gene and again found no influence of the jazD genotype.

To obtain further evidence that might support our preliminary results that JAZ proteins are not required for the repressive action of COI1 on PGM and PRLIP2 expression, we designed an alternative strategy. The idea was to complement coi1-t with a mutant COI1 protein that would be hampered in its interaction with JAZ proteins. To this aim, we made use of the known crystal structure of the complex formed between COI1 and the 20-amino acid (aa) JAZ1 degron in the presence of JA-lle (Sheard et al., 2010). The JAZ degron, which is shared between all JAZ proteins, has a bipartite structure with a six-aa loop region trapping the hormone in its binding pocket and a short helix that serves as a low-affinity anchor for docking the JAZ degron on COI1. Since the data obtained with the aos mutant already showed that hormone-mediated stabilization of the interaction between COI1 and the loop region of JAZs is not required for the repressive COI1 function, we decided to mutate amino acids interacting with the docking helix. It is shown that mutation of Tyr302 results in reduced COI1JAZ interactions in yeast (Sheard et al., 2010). To disturb the interaction more efficiently, we additionally mutated Glu203, which forms a hydrogen bridge to Lys215 in the JAZ1 docking helix $\left(\mathrm{COI}_{\mathrm{AA}}\right.$; Figure S10 shows the wildtype situation). As expected, the coronatine-induced interaction of $\mathrm{COI}_{\mathrm{AA}}$ with JAZ1, 2, 3, 9, and 12 fell below the level of detection in a yeast two-hybrid system (Figure S11).

Next, we tested for COl1-mediated activation of the $J A Z 1$ promoter in transiently transformed coi1-t 
protoplasts, which are competent to produce JA-Ile (Li et al., 2019). As expected, expression of COI1 ${ }_{W T}$ led to the induction of luciferase activity. In contrast, $\mathrm{COI} 1_{\mathrm{AA}}$ was far less efficient. This supports the notion that the interaction between $\mathrm{COI}_{\mathrm{AA}}$ and JAZs is severely impaired (Figure S12).

Subsequently, we generated transgenic coi1-t plants constitutively expressing COI1 cDNA with an HA tag preceding the open reading frame. Unexpectedly, the wildtype HA-COI1 protein did not efficiently repress $P G M$ and $P R L I P 2$ expression. The expression of $P R L I P 2$, for instance, was still 26-fold higher in complementation line \#2 than in the wild type, while being only 2.6-fold lower as compared to the empty vector control. In contrast, basal JAZ10 expression was 22-fold higher in this line than in plants transformed with the empty vector (Figure 4). This result indicates that HA-COI1 can efficiently activate JAZ10 expression, but that it can only barely fulfill the repressive function of COI1. This finding already indicates that COI functions in a manner that is different from its known mechanism of action when operating as a JA-lleindependent repressor.

Taking into account that the N-terminal tag or expression from a cDNA sequence could compromise the JA-Ileindependent $\mathrm{COI} 1$ function, we generated genomic $\mathrm{COI}$ clones with $\mathrm{C}$-terminal tags. At least with regard to male fertility, the functionality of such a construct has been reported before (Jewell and Browse, 2016). COI1 $W T$ and $C O I 1_{A A}$ constructs were transformed into the coi1- $t$ mutant and transgenic lines were selected based on similar $\mathrm{COI}_{\mathrm{WT}}$ and $\mathrm{COI}_{\mathrm{AA}}$ protein levels. First, we tested woundinduced activation of VEGETATIVE STORAGE PROTEIN 2
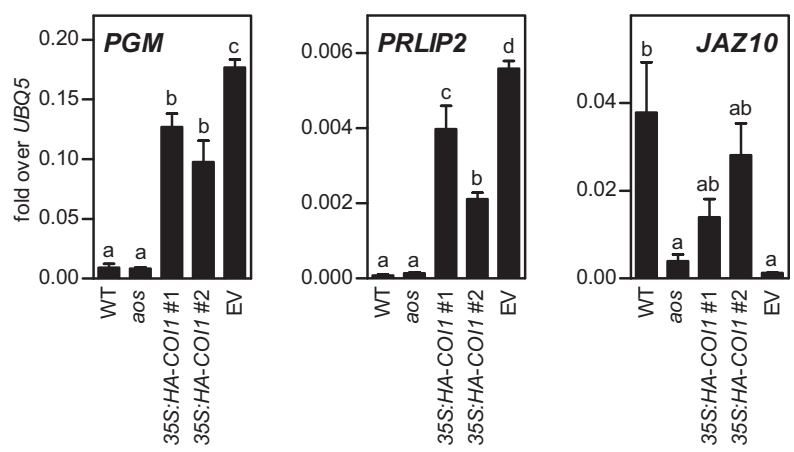

Figure 4. A 35S:HA-COI1 construct complements the canonical COI1 function more efficiently than the JA-lle-independent function.

$P G M, P R L I P 2$, and JAZ10 transcript levels, measured by qRT-PCR, in wildtype, aos, coi1-t/35S:HA-CO1, and coi1-t/EV (EV = empty vector) plants. RNA was extracted from untreated roots of seedlings grown on $1 / 2$ MS plates for 20 days with subsequent ( 5 days) cultivation on agarose in the absence of any added nutrients. Bars show the mean \pm SEM of three to four replicates with 40 (23 for EV) roots per replicate. For statistical analysis, one-way ANOVA was performed followed by Tukey's multiple comparison test; lowercase letters denote significant differences between samples $(P<0.05)$.
(VSP2), which is often used as a marker gene representing the response to JA-Ile- and COI1-dependent signaling processes. As expected, VSP2 expression was not induced in the segregating coi1- $t$ lines and a significant induction was observed in the two $\mathrm{COI}_{W T}$ complementation lines (Figure 5a). Plants harboring $\mathrm{COI}_{A A}$ barely responded to the wounding stimulus. $\mathrm{COI}_{A A}$ expression lines \#24 and \#55 had somewhat lower COI1 protein levels than the two control lines (Figure $5 \mathrm{~b}$ ), which might contribute to the weaker induction of VSP2 expression. Still, line \#44, which has similar or slightly higher COI1 levels as compared to the two control lines, showed lower VSP2 expression. We therefore conclude that $\mathrm{COI}_{\mathrm{AA}}$ complements the canonical COI1 functions less efficiently than COI1 ${ }_{\mathrm{WT}}$. This is supported by the observation that fertility is only restored in plants expressing $C O 11_{W T}$, while coi1-t/COI1 $A A$ plants resemble sterile coi1-t plants and do not produce seed pods (Figure S13).

In roots, differences between COI1 protein levels were less pronounced than in shoots (Figure $5 \mathrm{~b}$ ). In both types of complementation lines $\left(\mathrm{CO} / 1_{W T}\right.$ and $\left.\mathrm{COI} 1_{A A}\right)$, expression of PGM and PRLIP2 was as low as in the segregating wildtype plants, while expression was high in the segregating coi1-t plants (Figure 5c). Altogether, our results show that $\mathrm{COI}_{\mathrm{AA}}$ is able to repress the two marker genes in roots almost as efficiently as COI1 $1_{\mathrm{WT}}$, but that it is far less efficient in the activation of canonical COI1 functions as part of the JA signaling cascade.

Having established that the interaction between COI1 and JAZs is most likely not important for repression of $P G M$ and PRLIP2, we expected that the JAZ-regulated transcription factors MYC2, MYC3, and MYC4 (FernandezCalvo et al., 2011) would not be involved in the regulation of PGM and PRLIP2 expression. Indeed, transcript levels of these genes were not altered in the myc2 myc3 myc4 triple mutant (Figure S14). In contrast, the myc2 myc3 myc4 mutant phenocopied the coi1 mutant with respect to JAZ10 expression. Likewise, EIN3 and EIL1, which are repressed by at least JAZ1 (Zhu et al., 2011), did not influence expression of the marker genes that are de-repressed in coi1 (Figure S14).

\section{MED25 is required for $P G M$ and $P R L I P 2$ expression}

Recently, it has been shown that COI1 is recruited to target promoters through its interaction with subunit 25 of the mediator complex (MED25) (An et al., 2017). In a similar fashion, MED25 might be involved in the JA-lleindependent repressor function by recruiting COI1 to promoters of genes such as PGM and PRLIP2. To address this option, we assessed the expression of two marker genes in the med25 mutant and its outcrossed wild type. Due to very low expression levels in the wild type, we tested SARD1 rather than PRLIP2. The expression of PGM and $S A R D 1$ was even lower in med25, indicating that MED25 is 

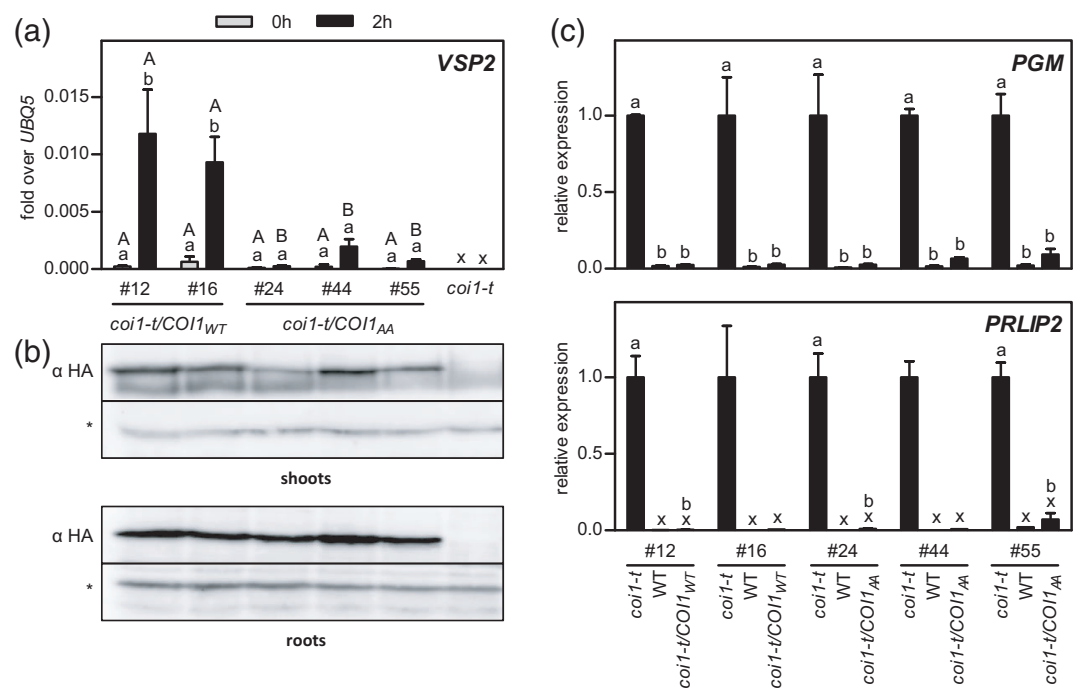

Figure 5. $C O I 1_{\mathrm{AA}}$ is less efficient than $\mathrm{COI} 1_{\mathrm{WT}}$ with respect to wound-induced $V S P 2$ expression but is similarly effective as a repressor of $P G M$ and $P R L I P 2$. (a) VEGETATIVE STORAGE PROTEIN 2 (VSP2) transcript levels were measured by qRT-PCR. RNA was extracted from untreated leaves at $0 \mathrm{~h}$ and at $2 \mathrm{~h}$ after wounding. Complementation lines are homozygous for the coi1-t allele and carry at least one copy of transgenic $\mathrm{COI}_{W T}$ or $\mathrm{COI}_{A A}$. Coi1- $t$ controls are a total of four plants with one plant segregated from each of the lines \#12,\#16,\#24, and \#55. Bars show the mean \pm SEM of two separately harvested leaves for each time point from three to four plants. For statistical analysis, two-way ANOva was performed followed by Bonferroni's multiple comparison test; lowercase letters denote significant differences within each line between $0 \mathrm{~h}$ and $2 \mathrm{~h}(P<0.05)$, uppercase letters denote significant differences between lines at the same time point $(P<0.05)$, and $x$ denotes that statistical analysis was not possible due to too many values falling below the detection threshold.

(b) Western blot of protein extracts obtained from shoots and roots from 39 to 402 -week-old seedlings of the segregating T2 generation of coi1-t/COI1 ${ }_{W T}$ or coi1-t/COI1 ${ }_{A A}$ complementation lines (extracts are loaded as indicated in (a)). C-terminally $3 \times \mathrm{HA}$-Strepll-tagged COI1 $\left(\mathrm{COI} 1_{\mathrm{WT}}\right.$ or $\left.\mathrm{COI} 1_{\mathrm{AA}}\right)$ protein levels were detected using an anti-HA antibody. The asterisk (*) depicts an unspecific band shown as loading control.

(c) PGM and PRLIP2 transcript levels, measured by qRT-PCR. RNA was extracted from roots of sand-soil-grown plants 10 days after mock treatment and subsequent transfer to soil. Three genotypes were obtained from the segregating offspring of each transgenic line: coi1-t mutants carrying the respective COI1 construct, wild type, and coi1- $t$ controls without the transgene. Values (normalized to reference gene UBQ5) from coi1- $t$ were set to 1.0. Bars show the mean \pm SEM of two to seven outcrossed coi1-t roots, two to four outcrossed WT roots, and seven to 13 coi1-t/COI1 $1_{W T}$ or $_{\text {coil-t/COI1 }}$ AA $_{\text {roots }}$ per transgenic line. For statistical analysis, one-way ANOVA was performed between the three genotypes segregated from one transgenic line each, followed by Tukey's multiple comparison test. In case of too many values falling below the detection limit as for PRLIP2 transcript levels (marked with $\mathrm{x}$ ), an unpaired two-tailed Student $t$-test was performed between coi1-t/COI1 $W T$ or coi1-t/COI1 $A A$ complementation lines and the respective outcrossed coi1- $t$ samples. Lowercase letters denote significant differences between samples $(P<0.05)$.

involved in their activation (Figure 6). Whether it also contributes to repression by recruiting COI1 can therefore not be concluded.

\section{DISCUSSION}

The plant hormone JA-lle controls both developmental and anti-stress programs (Wasternack and Hause, 2013). JA-lle facilitates the interaction of the receptor COI1 with transcriptional repressors (JAZs), which leads to JAZ degradation and activation of gene expression. Consistently, JA-lle-controlled processes like wound- or pathogen-induced gene expression, fertility, and growth are affected in the receptor mutant coi1 and the biosynthesis mutant aos. In contrast, tolerance of Arabidopsis against the vascular pathogens $V$. longisporum and $F$. oxysporum is observed in coi1 but not in aos plants (Ralhan et al., 2012; Thatcher et al., 2009). In this study we identified target genes of the JA-lle-independent COI1 function and we used these to demonstrate that COI1 can negatively affect a set of genes through a mechanism that does not seem to require known components of the JA-lle signaling pathway (Figure 7).
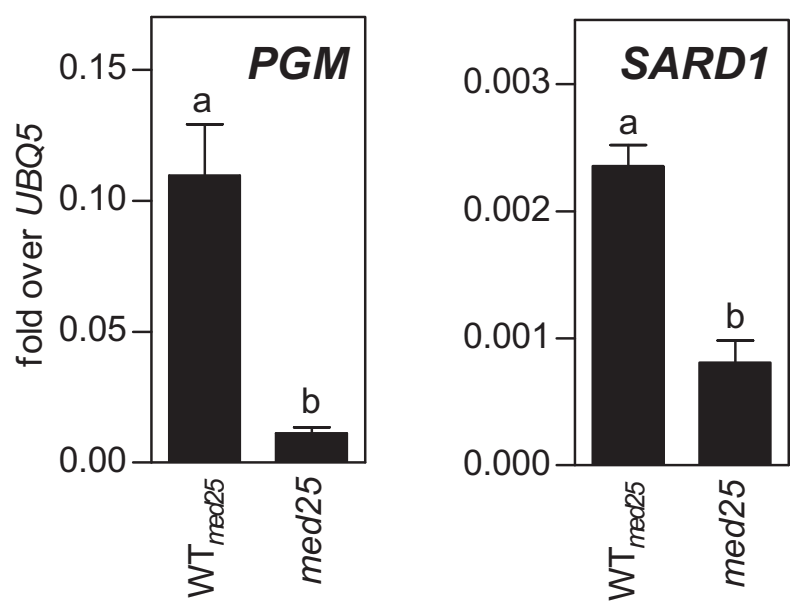

Figure 6. MED25 is required for expression of $P G M$ and SARD1.

$P G M$ and SARD1 transcript levels, measured by qRT-PCR. RNA was extracted from sand-soil-grown roots 21 days after mock treatment and subsequent transfer to soil. Bars show the mean \pm SEM of seven to eight roots per genotype. $\mathrm{WT}_{\text {med } 25}$ is the wild type obtained from the segregating offspring of heterozygous med 25 seeds. For statistical analysis, an unpaired two-tailed Student $t$-test was performed; lowercase letters denote significant differences between samples $(P<0.05)$. 

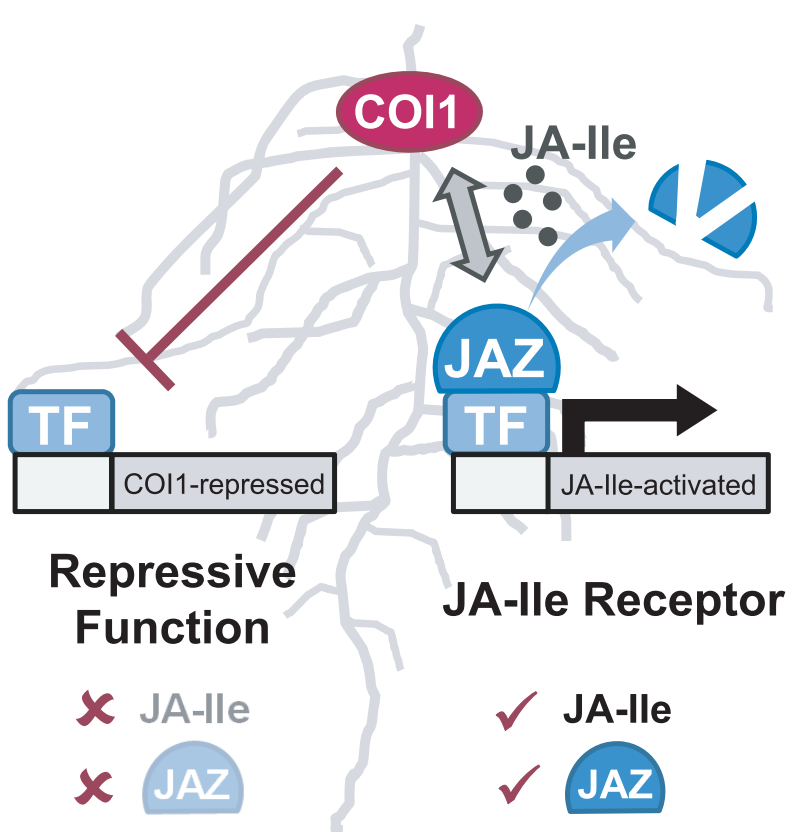

Figure 7. COI1 represses genes through a mechanism that does not involve JA-lle and most likely no JAZ repressor proteins.'

Constitutive de-repression of SA-related genes in coi1 roots is different from the JA-mediated repression of the SA pathway observed in leaves

The most conspicuous difference between the transcriptomes of coil versus aos or wild-type roots is the large number of SA-related genes that are de-repressed in coi1 (Table S1). From the two SA biosynthesis pathways known to operate in A. thaliana (Huang et al., 2010; Wildermuth et al., 2001), only genes of the isochorismate pathway (ICS1 and avrPphB SUSCEPTIBLE3 [PBS3]) (Rekhter et al., 2019; Wildermuth et al., 2001) are more highly expressed in coi1, leading to the hypothesis that increased SA synthesis through the activation of the isochorismate pathway is instrumental for increased expression of the whole group of SA-related genes. However, analysis of gene expression in the coi1 sid2 double mutant demonstrated that derepression of the two marker genes was detected even in the absence of elevated ICS1 transcript levels.

The negative effect of COI1 on SA-related genes in roots is different from the well-known inhibition of the SA pathway that occurs in leaves. In leaves, repression of the SA pathway is only observed when COI1 is activated by the bacterial JA-lle mimic coronatine (Kloek et al., 2001). Reduction of pathogen-induced SA levels is brought about by a mechanism that requires coronatine, COI1, and MYC2 (Zheng et al., 2012). Hence, in contrast to the situation in roots, the SA pathway in leaves is not constitutively activated, but it is hyper-activated after induction. Both activation of the SA pathway by Psm ES4326 and reduced growth of the pathogen were reverted to wild-type levels in coi1 expressing the SA-degrading enzyme NahG (Kloek et al., 2001). When we interfered with elevated SA synthesis in coi1 sid2 plants, increased expression of the COI1repressed marker genes and increased tolerance towards V. longisporum was still observed (Figure 3).

Nevertheless, it is notable that COI1 constitutively represses SA-related genes in roots. Many of these genes (e.g., ENHANCED DISEASE SUSCEPTIBILITY1 [EDS1], PHYTOALEXIN DEFICIENT4 [PAD4], SARD1, ICS1, PBS3, FLA VIN MONOOXYGENASE1 [FMO1], and AGD2-LIKE DEFENSE RESPONSE PROTEIN 1 [ALD1]) play crucial roles in the immune response systemic acquired resistance (Navarova et al., 2012). The transcription factor SARD1 is essential for the biosynthesis of the two signaling molecules $N$-hydroxy-pipecolic acid and SA (Sun et al., 2015), with ICS1/PBS3 and ALD1/FMO1 being important enzymes in the respective biosynthesis pathways (Hartmann and Zeier, 2018; Navarova et al., 2012; Rekhter et al., 2019; Wildermuth et al., 2001). Transcript profiling placed the SARD1-dependent section of genes downstream of the EDS1/PAD4 immune complex (Wagner et al., 2013; Wang et al., 2008). It can be speculated that inappropriate upregulation of this pathway might interfere with the composition of the microbiome in the rhizosphere, making an extra layer of repression necessary. It remains to be explored whether COI1 is a constitutive repressor or whether repression can be lifted on demand.

Since growth of $V$. longisporum or $F$. oxysporum is not inhibited in the root (Ralhan et al., 2013; Thatcher et al., 2009) and since the pathway is not constitutively activated in coil shoots (Kloek et al., 2001), a contribution to tolerance can only be assumed under the premises that extracellular defense compounds travel from the root to the shoot, where they might accumulate to interfere with fungal growth. It remains to be elucidated whether the tolerance phenotype can be reverted to susceptibility by suitable mutations of the above-mentioned regulators in the coi1 background.

\section{The repressive COI1 function is most likely independent of JAZ repressor proteins}

The high expression of 167 genes in coi1 roots might be explained by the accumulation of (certain) JAZs which would interfere with the action of a transcriptional repressor of this group of genes. As discussed below, the following pieces of evidence suggest that the repressive COI1 function is not due to the accumulation of JAZ proteins and thus acts through a different mechanism. (i) JA-lle, which is required for mediating the interaction between COI1 and JAZs, is not required for the repression (Figure 1). (ii) None of the JAZ proteins can be inactivated by COI1 in the aos background, indicating that ligandindependent degradation of specific JAZs is unlikely 
(Figure S7). (iii) The repression is mediated by a $\mathrm{COI}_{\mathrm{AA}}$ mutant protein that can only weakly interact with JAZs (Figure 5). (iv) An N-terminal HA-tag interferes more strongly with the repressive than with the canonical function (Figure 4).

Although JA-lle is required for the interaction between COI1 and JAZs, a JAZ1-GUS fusion protein can be degraded in the eATP-treated aos mutant. eATP treatment consequently activates known genes of the JA pathway (Tripathi et al., 2018). We consider this scenario to be unlikely in untreated roots since the affected target genes are different from those of the classical response. Furthermore, upon using a functional assay to assess COI1 activity in protoplasts, we did not get any evidence for ligandindependent degradation of specific JAZs (Figure S7).

$\mathrm{COI}_{\mathrm{AA}}$ has a weaker affinity to JAZs due to mutations in amino acids that stabilize the interaction between the docking helix of the JAZ degron and COI1 (Figures S10 and S11). Complementation of the coi1 mutant with this protein resulted in plants showing reduced VSP2 expression after wounding (Figure 5a). According to the accepted model of JA signaling through COI1, lower VSP2 expression is due to inefficient degradation of JAZs by SCF ${ }^{\text {CO1AA }}$. Since JA-lle levels are elevated upon wounding, residual $\mathrm{COI}_{\mathrm{AA}} / \mathrm{JAZ}$ interactions might occur. It is likely that at low JA-lle levels in non-wounded roots, complex formation between $\mathrm{COI}_{\mathrm{AA}}$ and JAZs is more affected. Hence, JAZ proteins might accumulate to similar or to only slightly lower levels in roots of coi1-t/COI1 ${ }_{A A}$ lines as compared to coi1-t. Still, transcription of PGM and PRLIP2 was strongly repressed despite the fact that JAZ proteins are stabilized. In combination with the data obtained with the aos mutant, we take this result as further evidence that JAZs do not take part in the regulation of COI1-repressed genes.

Plants expressing the non-degradable JAZ protein JAZ1 $\triangle 3 A$-GUS turned out to be not valuable for our research since even JAZ10 expression, which should be as low as in coi1, was not strongly affected (Figure S8). In jazD, which lacks 10 out of the 13 JAZs (Guo et al., 2018), strong repression would be expected if repressor activity was enhanced in the absence of JAZs (Figure S9). However, no significant repression was detected, leaving the only option that JAZ8, JAZ11, or JAZ12 might be JA-lleindependent substrates of COI1. However, no evidence for this was found in transient assays (Figure S7).

\section{Is COI1 a moonlighting protein?}

Moonlighting proteins perform multiple functions, which differ mechanistically (Huberts and van der Klei, 2010). Well-known examples are glycolytic enzymes. Arabidopsis glycerin aldehyde 3-phosphate dehydrogenase (GAPDH), for example, promotes transcriptional activation by interacting with the transcription factor nuclear factor $Y$ subunit C10 (NF-YC10) and enhancing binding to its target promoters (Kim et al., 2020). A decisive criterion for a moonlighting protein is the independency of both functions, meaning that inactivation of one of the functions should not affect the second function and vice versa. Adding an N-terminal tag to COI1 might have disturbed the potential moonlighting function (Figure 4) but not the JAIle receptor function, while mutating amino acids Glu203 and Tyr302 interfered with the receptor function but not with the potential moonlighting function (Figure 5). When acting as a JA-lle receptor, COI1 operates as a liganddependent F-box protein in an E3 ligase complex. The mechanism of action of its potential moonlighting activity remains to be elucidated. It can be envisioned that COI 1 is recruited to the chromatin where it might act as a scaffold for the assembly of a repressive complex. Alternatively, COI1 might act as a JA-lle-independent F-box protein that mediates the degradation of, e.g., a transcriptional activator. In this case, the label moonlighting would be debatable. Further studies are required to solve this question.

\section{EXPERIMENTAL PROCEDURES}

\section{Plant material}

All plants used in this study are in the $A$. thaliana Col-0 background. Mutant Arabidopsis lines were obtained from the following sources: aos (SALK_017756) and pft1-3 (med25, SALK_059316) (Kidd et al., 2009) from the Nottingham Arabidopsis Stock Centre (NASC); coi1-t (SALK 035548) (Mosblech et al., 2011) from I. Heilmann (Martin-Luther-University, Halle, Germany); coi1-1 (Xie et al., 1998) and coi1-16 (Ellis and Turner, 2002) from J. Turner (University of East Anglia, Norwich, UK); sid2-2 (Wildermuth et al., 2001) from F. M. Ausubel (Harvard University, Boston, USA); jazD (Guo et al., 2018) from G. Howe (Michigan State University, Michigan, USA); myc2 myc3 myc4 (Fernandez-Calvo et al., 2011) from R. Solano (National Centre for Biotechnology, Madrid, Spain); and eil1-1 ein3-1 (Alonso et al., 2003) from R. Vierstra (University of Wisconsin, Madison, USA). The coi1-t aos (Koster et al., 2012) and coi1-1 sid2-2 (this work) double mutants were generated by crossing the respective genotypes. Primers used for genotyping of the different alleles are given in Table S2. The identity of the eil1-1 ein3-1 mutant was verified by the lack of the triple response (Alonso et al., 2003) and the identities of coi1-16 and myc2 myc3 myc 4 by compromised JAZ10 expression.

\section{Plant growth conditions and treatments}

For RNA-seq analysis, each experiment started with 320 seeds obtained from heterozygous aos and coi1-t plants, respectively. Surface-sterilized seeds were sown onto vertical agar plates $(10 \times 10 \mathrm{~cm}, 20$ seeds per plate) containing half-strength Murashige-Skoog (MS) medium supplemented with $1 \%$ sucrose and kept at $4^{\circ} \mathrm{C}$ in darkness for $48-72 \mathrm{~h}$. Subsequently, plates were transferred to short day conditions (8-h day/16-h night cycle) at $22^{\circ} \mathrm{C}, 60 \%$ relative humidity, and a photon flux density of $80-100 \mu \mathrm{mol} \mathrm{m} \mathrm{m}^{-2} \mathrm{~s}^{-1}$. The lower parts of the plates were covered with aluminum foil to keep roots in semi-darkness. After 3 weeks, plantlets were transferred for $24 \mathrm{~h}$ onto vertical plates containing agarose ( $1 \%$ in water) in order to reduce saprophytic growth of the fungus. Roots were sprayed with $10^{5}$ spores $/ \mathrm{mL}$ tap water or only with water (mock). After 4 more days under the 
conditions mentioned above, roots and shoots were individually harvested with shoots yielding DNA for determination of the genotype and roots yielding RNA for transcriptome analysis. In detail, 33-36 roots of the two wild types, the homozygous aos, and the homozygous coi1-t plants, respectively, were combined for one RNA preparation. This experimental setup was repeated twice to obtain three independent biological replicates per genotype per treatment. RNAs from in total 24 samples (four genotypes, two treatments, three replicates) were used to construct libraries for Illumina sequencing. The same setup was used for qRT-PCR analysis (Figures 1, 2, and 4; Figures S3, S5, S6, S8, and S14). Here, between 20 and 50 roots were combined for one replicate.

For analysis of gene expression (Figures 3, 5, and 6; Figure S9) and for fungal infection (Figure 3 ), surface sterilized seeds were sown onto horizontal MS agar plates supplemented with $2 \%$ sucrose and grown in the same short day conditions described above. After 14 days, plantlets were transferred onto a 1:1 mixture of sand (white, 1-2 mm grain size, Rosnerski, Königslutter, Germany) and steamed soil (Fruhstorfer Erde, Spezial Substrat, Typ T, Str. 1 fein, HAWITA, Vechta, Germany) on a thin layer of Seramis (Westland Deutschland, Mogendorf, Germany) and grown for another 14 days under short day conditions at 120 $140 \mu \mathrm{mol}$ photons $\mathrm{m}^{-2} \mathrm{~s}^{-1}$. The sand-soil mixture was initially watered with $0.1 \%$ Wuxal Super (Manna, Ammerbuch-Pfäffingen, Germany) in $\mathrm{dH}_{2} \mathrm{O}$. For the first week, plants were kept under a transparent hood. If genotyping was required, a single leaf was clipped from each plant during the first week of growth on the sand-soil mixture. Plants were carefully uprooted from the sandsoil mixture and washed in tap water. Roots were then dipped in spore suspension $\left(1 \times 10^{6}\right.$ spores $/ \mathrm{mL}$ tap water $)$ or tap water (mock) for $45 \mathrm{~min}$, after which plants were planted into individual pots containing steamed soil (Fruhstorfer Erde, Spezial Substrat, Typ T, Str. 1 fein, HAWITA, Vechta, Germany) soaked with $0.2 \%$ Wuxal Super, where plants were kept for a final 10 to 21 days in short day conditions at $120-140 \mu \mathrm{mol}$ photons $\mathrm{m}^{-2} \mathrm{~s}^{-1}$ until harvest. During the first 2 days after transfer of plants to soil, they were kept under transparent hoods. A rootstock of one single plant was harvested for one biological replicate. For gene expression analysis in roots of untreated soilgrown plants (Figure S4), seeds were directly placed on soil, subjected for 2 days to cold treatment, and cultivated in a growth cabinet at $22^{\circ} \mathrm{C}$ in short day conditions at $120 \mu \mathrm{mol}$ photons $\mathrm{m}^{-2} \mathrm{~s}^{-1}$ and $60 \%$ relative humidity. After 5 weeks, plants were uprooted and roots were washed in tap water, after which they were frozen in liquid nitrogen. For wounding experiments, two leaves of 4.5-week-old plants grown under long day conditions (16-h day $/ 8$-h night cycle, $22^{\circ} \mathrm{C} / 18^{\circ} \mathrm{C}, 100 \mu \mathrm{mol}$ photons $\mathrm{m}^{-2} \mathrm{~s}^{-1}$ ) were cut at the petiole and immediately frozen in liquid nitrogen. Two further leaves of the same plant were wounded with forceps without damaging the mid rib. Leaves were collected separately after $2 \mathrm{~h}$. Subsequently, plants were further grown to assess their capacity to develop seed pods (Figure S8).

\section{Other methods}

For RNA-seq analysis (Methods S1), qRT-PCR (Methods S2), Western blot analysis (Methods S2), construction of recombinant plasmids (Methods S3), generation of transgenic plants (Methods S3), fungal culture (Methods S4), leaf area measurement (Methods S5), protoplast preparation/transfection (Methods S6), and yeast two-hybrid analysis (Methods S7), see detailed protocols in the Supporting Information. Appendix 1 displays the sequence of $\mathrm{pB}-\mathrm{GW}-\mathrm{HAS7}$ used to express $\mathrm{COI} 1$ and $\mathrm{COI}_{\mathrm{AA}}$ in transgenic plants.

\section{Statistical analyses}

Statistical analyses were performed with GraphPad Prism 5.0 (GraphPad Software, Inc., San Diego, CA). In order to not distort the statistical analysis for VSP2 expression in leaves (two-way analysis of variance [ANOVA]) by disregarding values that fell below the detection threshold, we corrected the values to ones orientated around the lowest value measured for that line and time point (two values for \#16 [0 h]; three values for \#24 [0 h]; three values for \#24 [2 h]; four values for \#44 [0 h]; and five values for \#55 $[0 \mathrm{~h}]$ ).

\section{Accession numbers}

Sequence data from this article can be found in The Arabidopsis Information Resource (http://www.arabidopsis.org/) under the following accession numbers: AOS (AT5G42650), COI1 (AT2G39940), ICS1 (AT1G74710), JAZ1 (AT1G19180), JAZ9 (AT1G70700), JAZ10 (AT5G13220), MED25 (AT1G25540), PGM (AT3G60415), PRLIP2 (AT5G24200), SARD1 (AT1G73805), UBO5 (AT3G62250), VSP2 (AT5G24770).

\section{ACKNOWLEDGMENTS}

We thank Ronald Scholz, Anna Hermann, and Kathi Dworak for excellent technical assistance, Dirk Paffenholz for help with characterization of transgenic plants, Chiara Trilling for performing protoplast experiments, and Natalie Leutert for help with the yeast two-hybrid experiments. We also thank the Transcriptome and Genome Analysis Laboratory (TAL) of the University Medical Center Göttingen (UMG) for performing the RNA-seq analysis. This work was funded by the Deutsche Forschungsgemeinschaft (GA330/24). Open Access funding enabled and organized by Projekt DEAL.

\section{CONFLICT OF INTEREST}

None of the authors has declared a conflict of interest.

\section{AUTHOR CONTRIBUTIONS}

LU and JS performed the experiments; CT designed and supervised the research and analyzed the RNA-seq data; CG designed the experiments and wrote the manuscript with input from all authors.

\section{DATA AVAILABILITY STATEMENT}

All relevant data can be found within the manuscript and its supporting materials.

\section{SUPPORTING INFORMATION}

Additional Supporting Information may be found in the online version of this article.

Figure S1. Venn diagrams showing the numbers and overlap of genes differentially expressed in mock- and $V$. longisporum-infected coi1-t roots ( $>$ twofold; $P<0.05$ ) as compared to aos and the two segregating wild-type lines.

Figure S2. Known genes regulated by the JA pathway are lowly expressed in aos and coi1-t.

Figure S3. PGM and PRLIP2 are de-repressed in coi1-1 and coi1-16 roots. 
Figure S4. $P G M$ and $P R L I P 2$ are de-repressed in coi1-t in untreated roots of soil-grown plants.

Figure S5. $P G M$ and $P R L I P 2$ are de-repressed in coi1 in the absence of ICS1-derived SA.

Figure S6. $P G M$ and $P R L I P 2$ are de-repressed in coi1 in the absence of AOS-derived JA-lle.

Figure S7. No COI1-dependent degradation of any JAZs is observed in the absence of JA-lle.

Figure S8. JAZ1 $\triangle 3 A$ :GUS plants only partially mimic the coi1-16 phenotype with respect to JAZ10 expression in roots.

Figure S9. PGM and SARD1 are not affected in jaz decuple plants.

Figure S10. Top view of the JAZ1 docking helix bound to COI1.

Figure S11. COI1 ${ }_{\mathrm{AA}}$ is impaired in mediating induction of the $J A Z 1$ promoter in protoplasts.

Figure S12. coi1- $t$ plants expressing $\mathrm{COI1}_{\mathrm{AA}}$ remain sterile.

Figure S13. COI1-mediated repression of PGM and PRLIP2 does not involve known JAZ-interacting transcription factors.

Figure S14. COI1-mediated repression of PGM and PRLIP2 does not involve known JAZ-interacting transcription factors.

Table S1. RNA-seq analysis.

Table S2. Primers for genotyping.

Table S3. Primers for qRT-PCR analysis.

Table S4. Primers for cloning.

Methods S1. RNA-seq analysis.

Methods S2. Quantitative reverse transcription PCR (qRT-PCR) and Western blot analysis.

Methods S3. Construction of recombinant plasmids and generation of transgenic plants.

Methods S4. Fungal culture.

Methods S5. Leaf area measurement

Methods S6. Assessment of COI1 activity in transiently transformed protoplasts.

Methods S7. Yeast two-hybrid analysis.

Appendix 1. Sequence of pB-GW-HAS7.

\section{REFERENCES}

Adams, E. \& Turner, J. (2010) COI1, a jasmonate receptor, is involved in ethylene-induced inhibition of Arabidopsis root growth in the light. Journal of Experimental Botany, 61, 4373-4386.

Alonso, J.M., Stepanova, A.N., Solano, R., Wisman, E., Ferrari, S., Ausubel F.M. et al. (2003) Five components of the ethylene-response pathway identified in a screen for weak ethylene-insensitive mutants in Arabidopsis Proceedings of the National Academy of Sciences USA, 100, 2992-2997.

An, C., Li, L., Zhai, O., You, Y., Deng, L., Wu, F. et al. (2017) Mediator subunit MED25 links the jasmonate receptor to transcriptionally active chromatin. Proceedings of the National Academy of Sciences USA, 114, E8930-E8939.

Brooks, D.M., Bender, C.L. \& Kunkel, B.N. (2005) The Pseudomonas syringae phytotoxin coronatine promotes virulence by overcoming salicylic acid-dependent defences in Arabidopsis thaliana. Molecular Plant Pathology, 6, 629-639.

Chini, A., Fonseca, S., Fernandez, G., Adie, B., Chico, J.M., Lorenzo, 0. et al. (2007) The JAZ family of repressors is the missing link in jasmonate signalling. Nature, 448, 666-671.

Ellis, C. \& Turner, J.G. (2002) A conditionally fertile coi1 allele indicates cross-talk between plant hormone signalling pathways in Arabidopsis thaliana seeds and young seedlings. Planta, 215, 549-556.

Fernandez-Calvo, P., Chini, A., Fernandez-Barbero, G., Chico, J.M. Gimenez-lbanez, S., Geerinck, J. et al. (2011) The Arabidopsis bHLH transcription factors MYC3 and MYC4 are targets of JAZ repressors and act additively with MYC2 in the activation of jasmonate responses. The Plant Cell, 23, 701-715.
Geng, Y., Wu, R., Wee, C.W., Xie, F., Wei, X., Chan, P.M. et al. (2013) A spatio-temporal understanding of growth regulation during the salt stress response in Arabidopsis. The Plant Cell, 25, 2132-2154.

Guo, Q., Yoshida, Y., Major, I.T., Wang, K., Sugimoto, K., Kapali, G. et al. (2018) JAZ repressors of metabolic defense promote growth and reproductive fitness in Arabidopsis. Proceedings of the National Academy of Sciences USA, 115, E10768-E10777.

Hartmann, M. \& Zeier, J. (2018) I-lysine metabolism to N-hydroxypipecolic acid: an integral immune-activating pathway in plants. The Plant Journal, 96, 5-21.

Huang, J., Gu, M., Lai, Z., Fan, B., Shi, K., Zhou, Y.H. et al. (2010) Functional analysis of the Arabidopsis PAL gene family in plant growth, development, and response to environmental stress. Plant Physiology, 153 1526-1538.

Huberts, D.H. \& van der Klei, I.J. (2010) Moonlighting proteins: an intriguing mode of multitasking. Biochimica et Biophysica Acta, 1803, 520-525.

Jakab, G., Manrique, A., Zimmerli, L., Metraux, J.P. \& Mauch-Mani, B. (2003) Molecular characterization of a novel lipase-like pathogeninducible gene family of Arabidopsis. Plant Physiology, 132, 2230-2239.

Jewell, J.B. \& Browse, J. (2016) Epidermal jasmonate perception is sufficient for all aspects of jasmonate-mediated male fertility in Arabidopsis. The Plant Journal, 85, 634-647.

Kidd, B.N., Edgar, C.I., Kumar, K.K., Aitken, E.A., Schenk, P.M., Manners, J.M. et al. (2009) The mediator complex subunit PFT1 is a key regulator of jasmonate-dependent defense in Arabidopsis. The Plant Cell, 21, 2237-2252.

Kim, S.C., Guo, L. \& Wang, X. (2020) Nuclear moonlighting of cytosolic glyceraldehyde-3-phosphate dehydrogenase regulates Arabidopsis response to heat stress. Nature Communications, 11, 3439.

Kloek, A.P., Verbsky, M.L., Sharma, S.B., Schoelz, J.E., Vogel, J., Klessig D.F. et al. (2001) Resistance to Pseudomonas syringae conferred by an Arabidopsis thaliana coronatine-insensitive (coi1) mutation occurs through two distinct mechanisms. The Plant Journal, 26, 509-522.

Koster, J., Thurow, C., Kruse, K., Meier, A., Iven, T., Feussner, I. et al. (2012) Xenobiotic- and jasmonic acid-inducible signal transduction pathways have become interdependent at the Arabidopsis CYP81D11 promoter. Plant Physiology, 159, 391-402.

Li, N., Uhrig, J.F., Thurow, C., Huang, L.J. \& Gatz, C. (2019) Reconstitution of the jasmonate signaling pathway in plant protoplasts. Cells, 8, 1532

McConn, M., Creelman, R.A., Bell, E., Mullet, J.E. \& Browse, J. (1997) Jasmonate is essential for insect defense in Arabidopsis. Proceedings of the National Academy of Sciences USA, 94, 5473-5477.

Mosblech, A., Thurow, C., Gatz, C., Feussner, I. \& Heilmann, I. (2011) Jasmonic acid perception by COI1 involves inositol polyphosphates in Arabidopsis thaliana. The Plant Journal, 65, 949-957.

Navarova, H., Bernsdorff, F., Doring, A.C. \& Zeier, J. (2012) Pipecolic acid an endogenous mediator of defense amplification and priming, is a critical regulator of inducible plant immunity. The Plant Cell, 24, 5123-5141.

Park, J.H., Halitschke, R., Kim, H.B., Baldwin, I.T., Feldmann, K.A. \& Feyereisen, R. (2002) A knock-out mutation in allene oxide synthase results in male sterility and defective wound signal transduction in Arabidopsis due to a block in jasmonic acid biosynthesis. The Plant Journal, 31 $1-12$.

Ralhan, A., Schottle, S., Thurow, C., Iven, T., Feussner, I., Polle, A. et al. (2012) The vascular pathogen Verticillium longisporum requires a jasmonic acid-independent COI1 function in roots to elicit disease symp toms in Arabidopsis shoots. Plant Physiology, 159, 1192-1203.

Ralhan, A., Thurow, C. \& Gatz, C. (2013) The tolerance of the Arabidopsis defense hormone receptor mutant coi1 against the vascular pathogen Verticillium longisporum is not due to increased levels of the active hormone jasmonoyl-isoleucine. Plant Signaling \& Behavior, 8, e27008.

Rekhter, D., Ludke, D., Ding, Y., Feussner, K., Zienkiewicz, K., Lipka, V. et al. (2019) Isochorismate-derived biosynthesis of the plant stress hormone salicylic acid. Science, 365, 498-502.

Sheard, L.B., Tan, X., Mao, H., Withers, J., Ben-Nissan, G., Hinds, T.R. et al. (2010) Jasmonate perception by inositol-phosphate-potentiated COI1JAZ co-receptor. Nature, 468, 400-405.

Stotz, H.U., Mueller, S., Zoeller, M., Mueller, M.J. \& Berger, S. (2013) TGA transcription factors and jasmonate-independent COI1 signalling regu late specific plant responses to reactive oxylipins. Journal of Experimental Botany, 64, 963-975. 
Sun, T., Zhang, Y., Li, Y., Zhang, Q., Ding, Y. \& Zhang, Y. (2015) ChIP-seq reveals broad roles of SARD1 and CBP60g in regulating plant immunity Nature Communications, 6, 10159.

Thatcher, L.F., Manners, J.M. \& Kazan, K. (2009) Fusarium oxysporum hijacks COI1-mediated jasmonate signaling to promote disease development in Arabidopsis. The Plant Journal, 58, 927-939.

Thines, B., Katsir, L., Melotto, M., Niu, Y., Mandaokar, A., Liu, G. et al. (2007) JAZ repressor proteins are targets of the SCF(COI1) complex during jasmonate signalling. Nature, 448, 661-665.

Tripathi, D., Zhang, T., Koo, A.J., Stacey, G. \& Tanaka, K. (2018) Extracellular ATP acts on jasmonate signaling to reinforce plant defense. Plant Physiology, 176, 511-523.

Vijayan, P., Shockey, J., Levesque, C.A., Cook, R.J. \& Browse, J. (1998) A role for jasmonate in pathogen defense of Arabidopsis. Proceedings of the National Academy of Sciences USA, 95, 7209-7214.

von Malek, B., van der Graaff, E., Schneitz, K. \& Keller, B. (2002) The Arabidopsis male-sterile mutant dde2-2 is defective in the ALLENE OXIDE SYNTHASE gene encoding one of the key enzymes of the jasmonic acid biosynthesis pathway. Planta, 216, 187-192.

Wagner, S., Stuttmann, J., Rietz, S., Guerois, R., Brunstein, E., Bautor, J. et al. (2013) Structural basis for signaling by exclusive EDS1 heteromeric complexes with SAG101 or PAD4 in plant innate immunity. Cell Host \& Microbe, 14, 619-630.

Wang, L., Mitra, R.M., Hasselmann, K.D., Sato, M., Lenarz-Wyatt, L., Cohen, J.D. et al. (2008) The genetic network controlling the Arabidopsis transcriptional response to Pseudomonas syringae pv. maculicola: roles of major regulators and the phytotoxin coronatine. Molecular PlantMicrobe Interactions, 21, 1408-1420.

Wasternack, C. \& Hause, B. (2013) Jasmonates: biosynthesis, perception, signal transduction and action in plant stress response, growth and development. An update to the 2007 review in Annals of Botany. Annals of Botany, 111, 1021-1058.

Wildermuth, M.C., Dewdney, J., Wu, G. \& Ausubel, F.M. (2001) Isochorismate synthase is required to synthesize salicylic acid for plant defence. Nature, 414, 562-565.

Xie, D.X., Feys, B.F., James, S., Nieto-Rostro, M. \& Turner, J.G. (1998) COI1 an Arabidopsis gene required for jasmonate-regulated defense and fertility. Science, 280, 1091-1094.

Yan, J., Yao, R., Chen, L., Li, S., Gu, M., Nan, F. et al. (2018) Dynamic perception of jasmonates by the F-box protein COI1. Molecular Plant, 11 1237-1247.

Zheng, X.Y., Spivey, N.W., Zeng, W., Liu, P.P., Fu, Z.Q., Klessig, D.F. et al. (2012) Coronatine promotes Pseudomonas syringae virulence in plants by activating a signaling cascade that inhibits salicylic acid accumulation. Cell Host \& Microbe, 11, 587-596.

Zhu, Z., An, F., Feng, Y., Li, P., Xue, L., A, M. et al. (2011) Derepression of ethylene-stabilized transcription factors (EIN3/EIL1) mediates jasmonate and ethylene signaling synergy in Arabidopsis. Proceedings of the National Academy of Sciences USA, 108, 12539-12544. 


\section{Figure S1}

Higher expressed in coi1-t

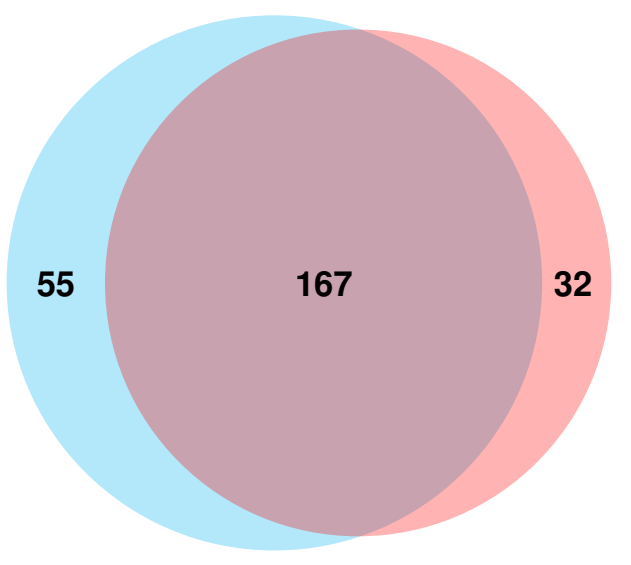

Lower expressed in coi1-t

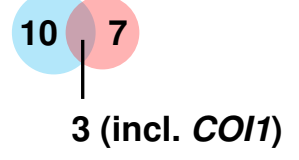

mock

4dpi

Figure S1. Venn diagrams showing the numbers and overlap of genes differentially expressed in mock- and $V$. longisporum-infected coi1-t roots $(>2$-fold; $p<0.05$ ) as compared to aos and the two segregating wild-type lines.

Expression data were obtained by RNAseq analysis of RNA extracted from roots of four genotypes (aos, coi1-t, and the two wild-types lines obtained from the segregating offspring of heterozygous aos and coi1- $t$ seeds) after mock treatment or infection with $V$. longisporum. Circles are drawn to scale with respect to the number of genes. 
Figure S2
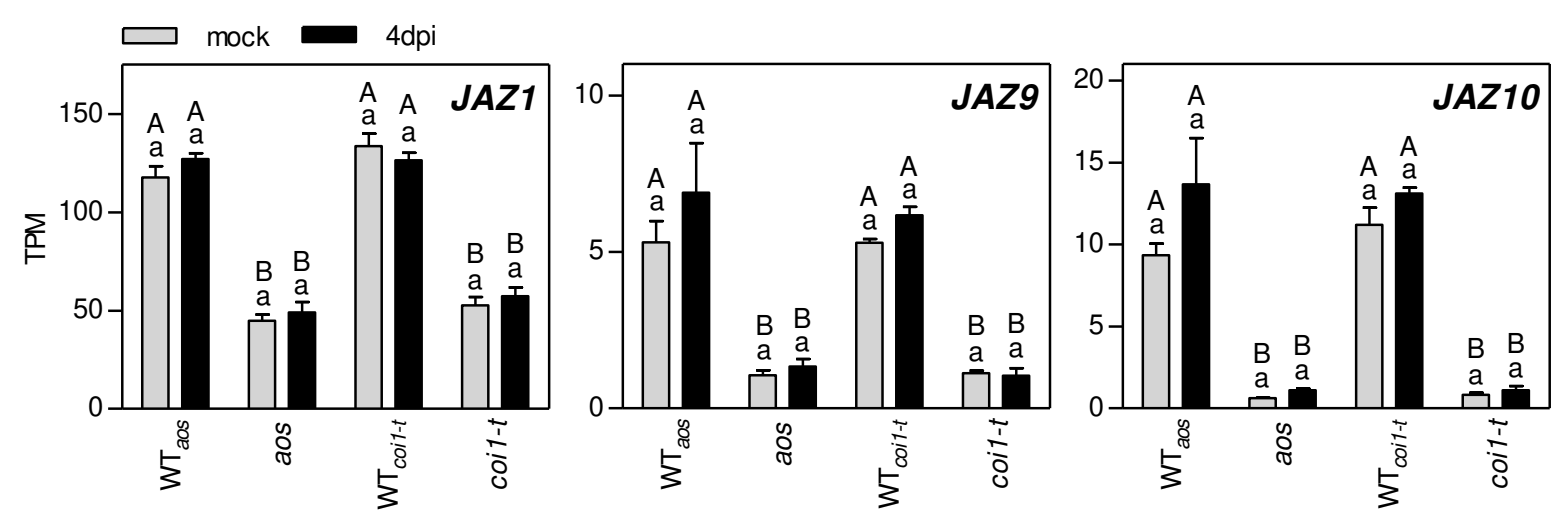

Figure S2. Known genes regulated by the JA pathway are less expressed in aos and coi1-t.

Relative expression of $J A Z 1, J A Z 9$ and $J A Z 10$ transcript levels as quantified by RNAseq analysis. Bars represent the average of Transcripts Per Million (TPM) \pm SEM of three biological replicates of each genotype, with each replicate representing 33 to 36 roots from one independent experiment. For statistical analysis, a two-way ANOVA was performed followed by Bonferroni's multiple comparison test; lowercase letters denote significant differences within each genotype between mock and $4 \mathrm{dpi}$ ( $p$ $<0.05$ ), uppercase letters denote significant differences between genotypes subjected to the same treatment $(p<0.05)$. $\mathrm{WT}_{\text {aos }}$ and $\mathrm{WT}_{\text {coit-t } t}$ are the two wild-types lines obtained from the segregating offspring of heterozygous aos and coir-t seeds. 
Figure S3
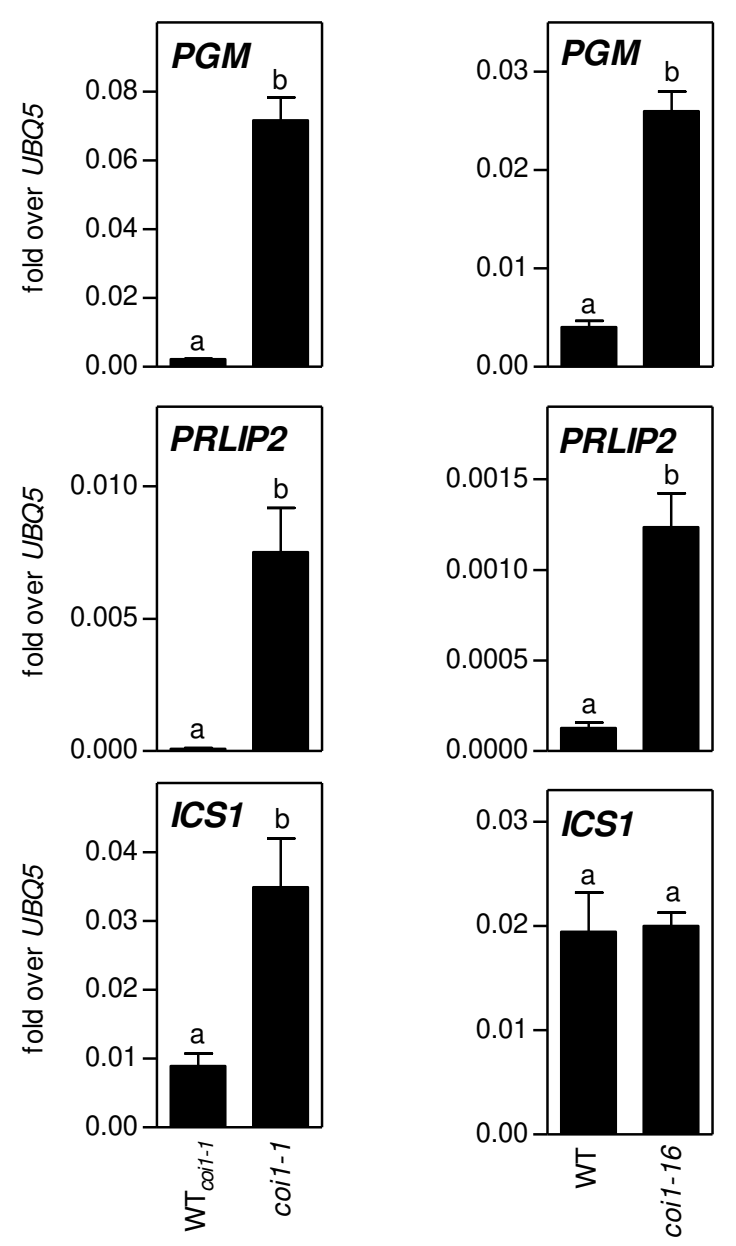

Figure S3. PGM and PRLIP2 are de-repressed in coi1-1 and coi1-16 roots.

PGM, PRLIP2 and ICS1 transcript levels, measured by qRT-PCR. RNA was extracted from mocktreated roots of seedlings grown on $1 / 2$ MS plates with subsequent cultivation on agarose in the absence of any added nutrients. Bars are means \pm SEM of three to four replicates with 20-23 roots per replicate. For statistical analysis, an unpaired Student's t-test (two-tailed) was performed between coi1 and the respective WT samples; lowercase letters denote significant differences between samples $(p<$ $0.05)$. $W T_{\text {coit-1 }}$ is the wild-type obtained from the segregating offspring of heterozygous coi1-1 seeds. 
Figure S4

(a)

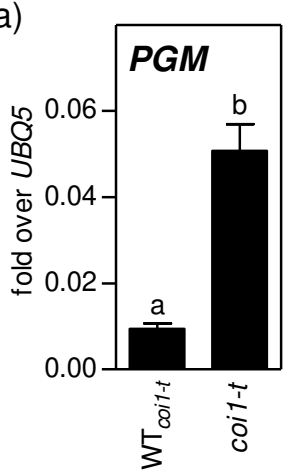

(b)

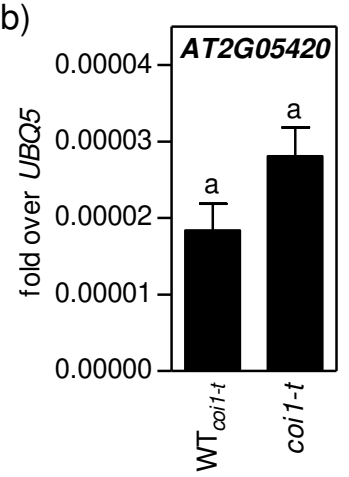

Figure S4. PGM and PRLIP2 are de-repressed in coi1-t in untreated roots of soil-grown plants.

(a) PGM and PRLIP2 transcript levels, measured by qRT-PCR. RNA was extracted from untreated roots of soil-grown 5-week old plants. Bars are means \pm SEM of twelve roots per genotype. $x$ indicates that for PRLIP2 expression in WT only three values are shown as the other nine fell below the detection threshold in our analysis. For statistical analysis, an unpaired Student's t-test (two-tailed) was performed between coi1-t and WT samples; lowercase letters denote significant differences between samples $(p<0.05)$. $W T_{\text {coit-t }}$ is the wild-type obtained from the segregating offspring of heterozygous coi1-t seeds. (b) AT2G05420 transcript levels, measured by qRT-PCR. RNA was extracted from untreated roots of soil-grown 5-week old plants. Bars are means \pm SEM of ten to twelve roots per genotype. For statistical analysis, an unpaired Student's t-test (two-tailed) was performed between coi1-t and WT samples; lowercase letters denote significant differences between samples $(p<0.05)$. $\mathrm{WT}_{\text {coit-t }}$ is the wild-type obtained from the segregating offspring of heterozygous coi1-t seeds. 
Figure S5
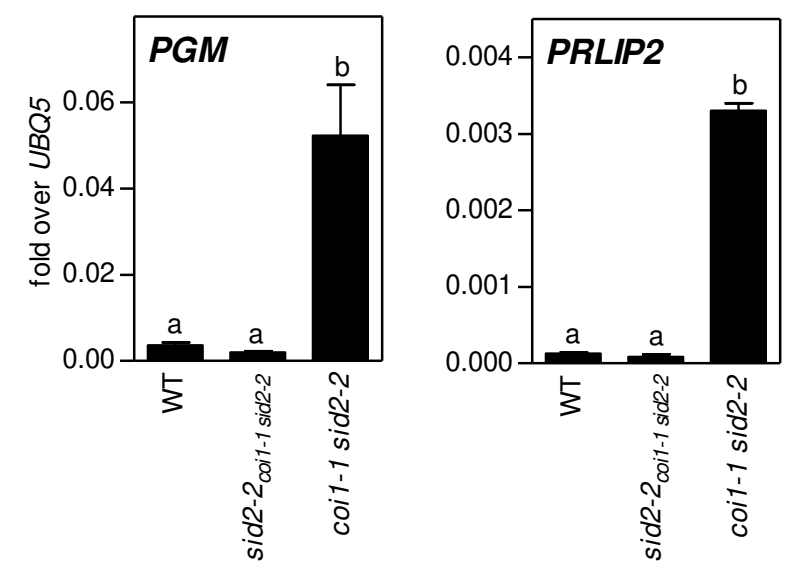

Figure S5. PGM and PRLIP2 are de-repressed in coi1 in the absence of ICS1-derived SA.

PGM and PRLIP2 transcript levels, measured by qRT-PCR. RNA was extracted from mock-treated roots of seedlings grown on $1 / 2$ MS plates with subsequent cultivation on agarose in the absence of any added nutrients. Bars are means \pm SEM of three to five replicates with 20-23 roots per replicate. For statistical analysis, a one-way ANOVA was performed followed by Tukey's multiple comparison test; lowercase letters denote significant differences between samples $(p<0.05)$. sid2-2 $2_{\text {coir- } 1 \text { sid2-2 }}$ are sid2-2 plants obtained from the segregating offspring of coi1-1 sid2-2 plants, which are heterozygous for the coi1-1 allele and homozygous for the sid2-2 allele. 
Figure S6
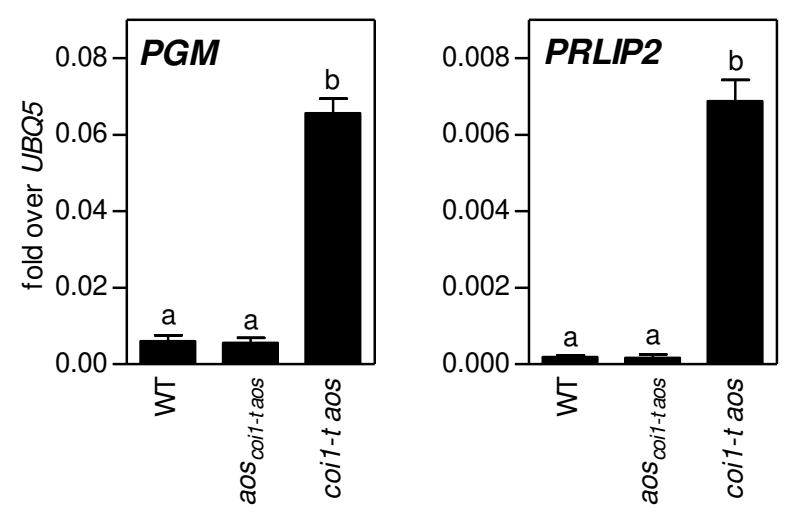

Figure S6. PGM and PRLIP2 are de-repressed in coi1 in the absence of AOS-derived JA-lle.

$P G M$ and PRLIP2 transcript levels, measured by qRT-PCR. RNA was extracted from mock-treated roots of seedlings grown on $1 / 2$ MS plates with subsequent cultivation on agarose in the absence of any added nutrients. Bars are means \pm SEM of three to four replicates with at least ten roots per replicate. For statistical analysis, a one-way ANOVA was performed followed by Tukey's multiple comparison test; lowercase letters denote significant differences between samples $(p<0.05)$. aos coirt $t$ aos are aos plants obtained from the segregating offspring of coi1-t aos plants, which are heterozygous for the coi1-t allele and homozygous for aos allele. 
Figure S7
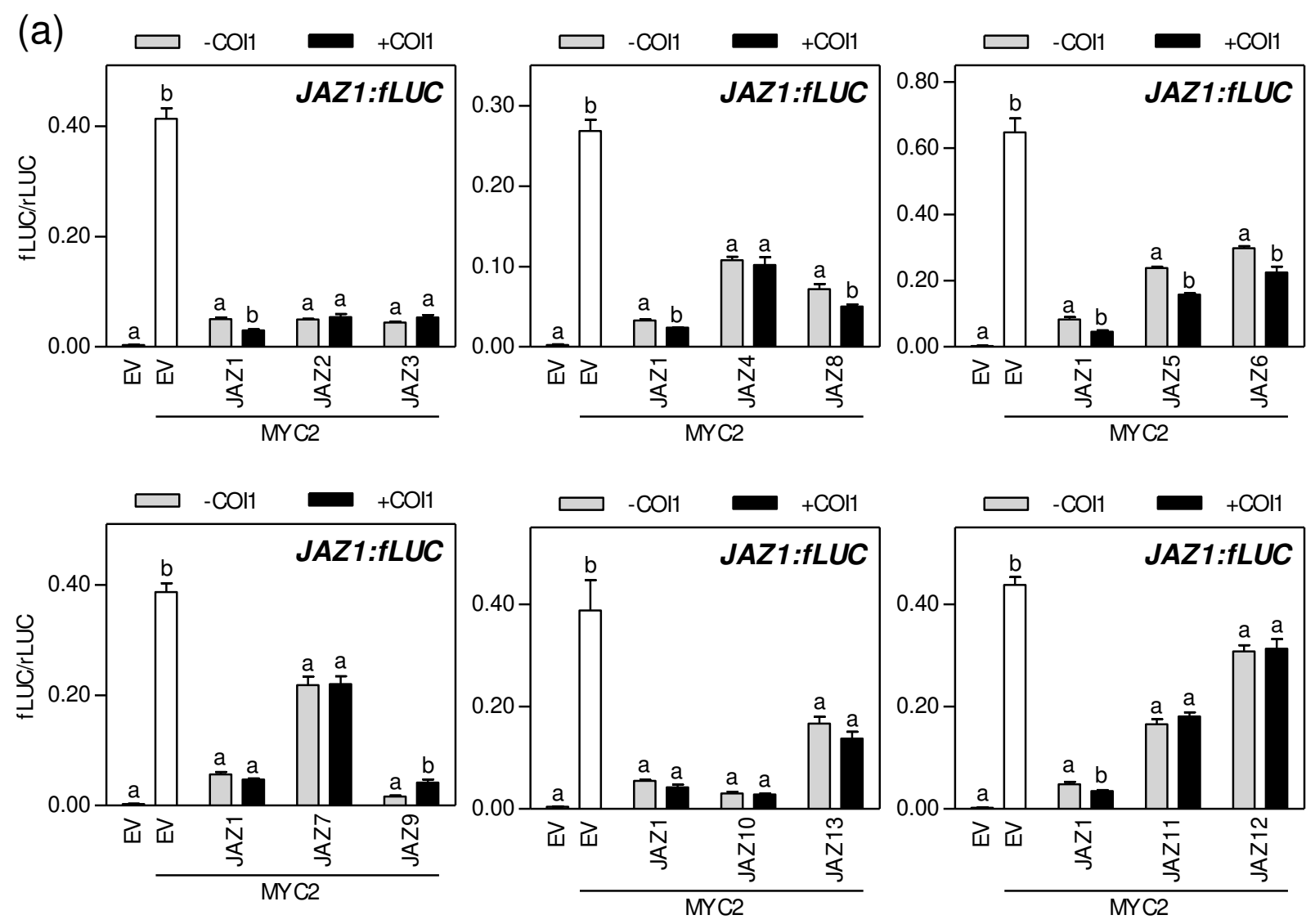

(b)

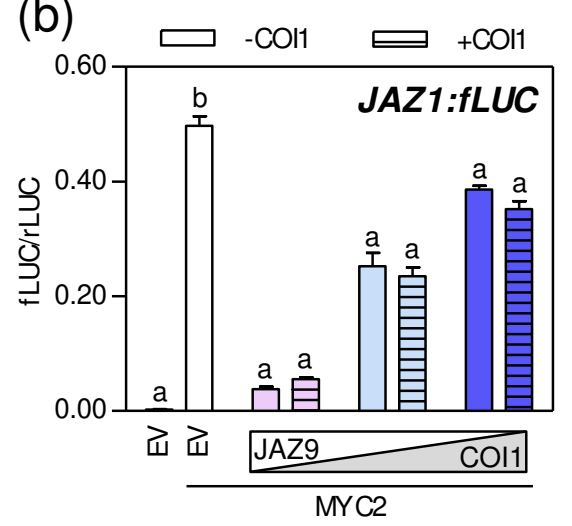

Figure S7. No COI1-dependent degradation of any JAZ is observed in the absence of Coronatine/JA-Ile.

Full Legend on next page. 
F igure S7. No COl1-dependent degradation of any JAZs is observed in the absence of JA-lle.

(a/b) Luciferase activities yielded by MYC2-activated JAZ1:fLuc in the presence or absence of cotransfected JAZs and COI1. Since JAZ9-mediated repression seemed to be relieved by COl1, the assay was repeated with different JAZ9/COI1 ratios in b.

Arabidopsis leaf protoplasts prepared from coi1-t aos mutant plants were cotransfected with the reporter plasmid $(3.5 \mu \mathrm{g})$ containing the firefly LUCIFERASE coding region ( $f L U C)$ driven by the JAZ1 promoter. Effector plasmids ( $3.5 \mu \mathrm{g}$ each per sample) contained the coding regions of MYC2, JAZ113 , and COI1 driven by the UBQ10 promoter. In b, different JAZ9/COI1 ratios were used (pink: $3.5 \mu \mathrm{g}$ $\mathrm{JAZ9}+3.5 \mu \mathrm{g} \mathrm{EV}$ or COl1; light blue: $1 \mu \mathrm{g} \mathrm{JAZ9} \mathrm{+} 6.1 \mu \mathrm{g} \mathrm{EV}$ or COl1; blue: $0.5 \mu \mathrm{g} \mathrm{JAZ9} \mathrm{+} 6.6 \mu \mathrm{g} \mathrm{EV}$ or COI1). Each sample contained $0.7 \mu \mathrm{g}$ of the plasmid pUBQ10-HA-rLUC encoding the Renilla LUCIFERASE ( $L L U C$ ) gene driven by the UBQ10 promoter The empty vector plasmid (EV) was added so that the amounts of transfected DNA was always $14.7 \mu \mathrm{g}$. Firefly luciferase (fLUC) activities were normalized to Renilla luciferase (rLUC) activities. Values represent means $( \pm S E)$ of four independently transformed batches of protoplasts. For statistical analysis, an unpaired Student's t-test (two-tailed) was performed between -COI1 and +COI1 values for each JAZ construct and between EV and EV + MYC2 controls; letters denote significant differences between samples $(p<0.05)$. 
Figure S8
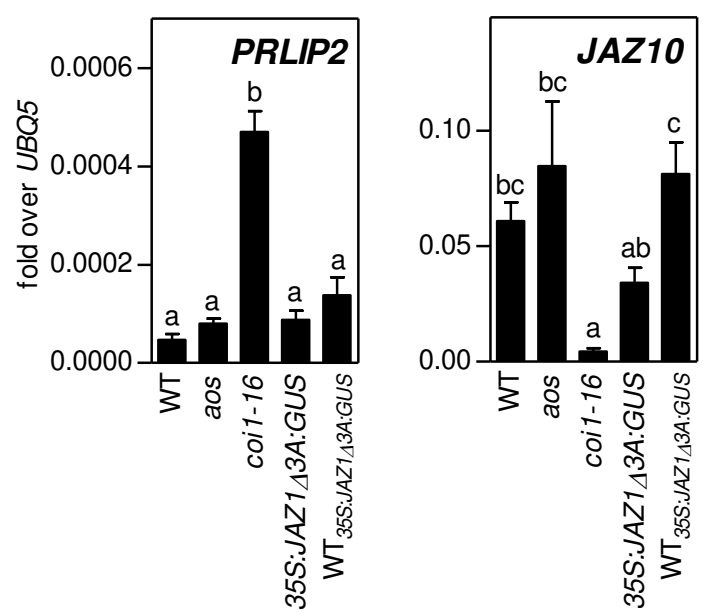

Figure S8. JAZ1 $\triangle 3 A$ :GUS plants only partially mimic the coi1-16 phenotype with respect to JAZ10 expression in roots.

PRLIP2 and JAZ10 transcript levels, measured by qRT-PCR. RNA was extracted from roots of seedlings grown vertically on $1 / 2$ MS plates for three weeks in short day conditions with subsequent cultivation on agarose for 5 days in the absence of any added nutrients. Seedlings were then sprayed with with $10 \mu \mathrm{M} \mathrm{MeJA}$ (in $\mathrm{H}_{2} \mathrm{O}$ with $0.0018 \% \mathrm{EtOH}$ ) and incubated for two hours. Bars are means \pm SEM of two to four replicates with 20-44 roots per replicate. WT $_{35 S: J A Z 1 \triangle 3 A: G U S}$ plants were obtained from the segregating population derived from the cross between the male sterile 35S:JAZ1 3 3A:GUS with pollen from wild-type plants. For statistical analysis, a one-way ANOVA was performed followed by Tukey's multiple comparison test; lowercase letters denote significant differences between samples $(p<0.05)$. 
Figure S9
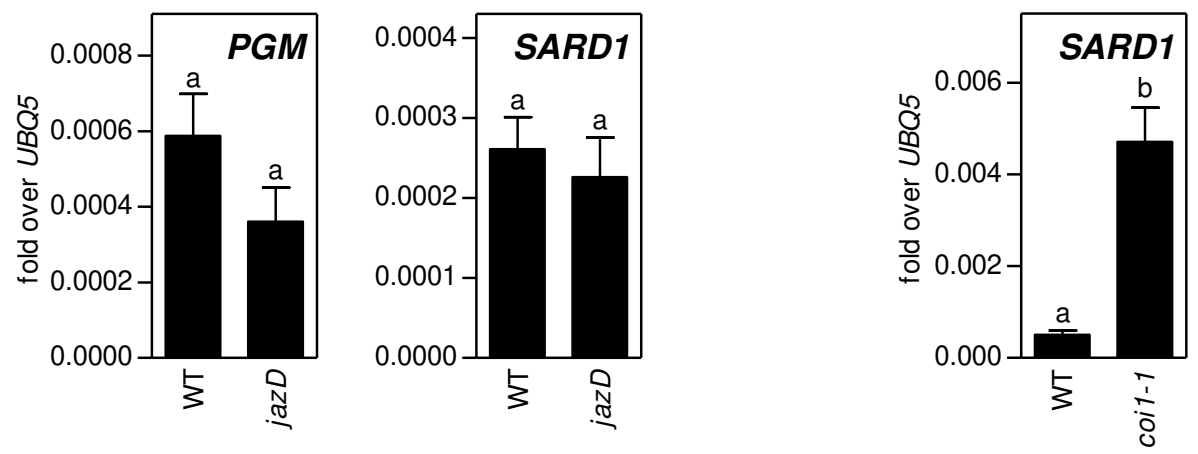

Figure S9. PGM and SARD1 are not affected in jaz decuple plants.

PGM and SARD1 transcript levels, measured by qRT-PCR. RNA was extracted from roots of sandsoil grown plants 10 days after mock treatment and subsequent transfer to soil. Bars are means \pm SEM of seven to eight replicates. The right panel demonstrates that SARD1 is de-repressed in coi1-1 under these experimental conditions. For statistical analysis, an unpaired Student's t-test (two-tailed) was performed between WT and mutant samples; lowercase letters denote significant differences between samples $(p<0.05)$. 
Figure S10

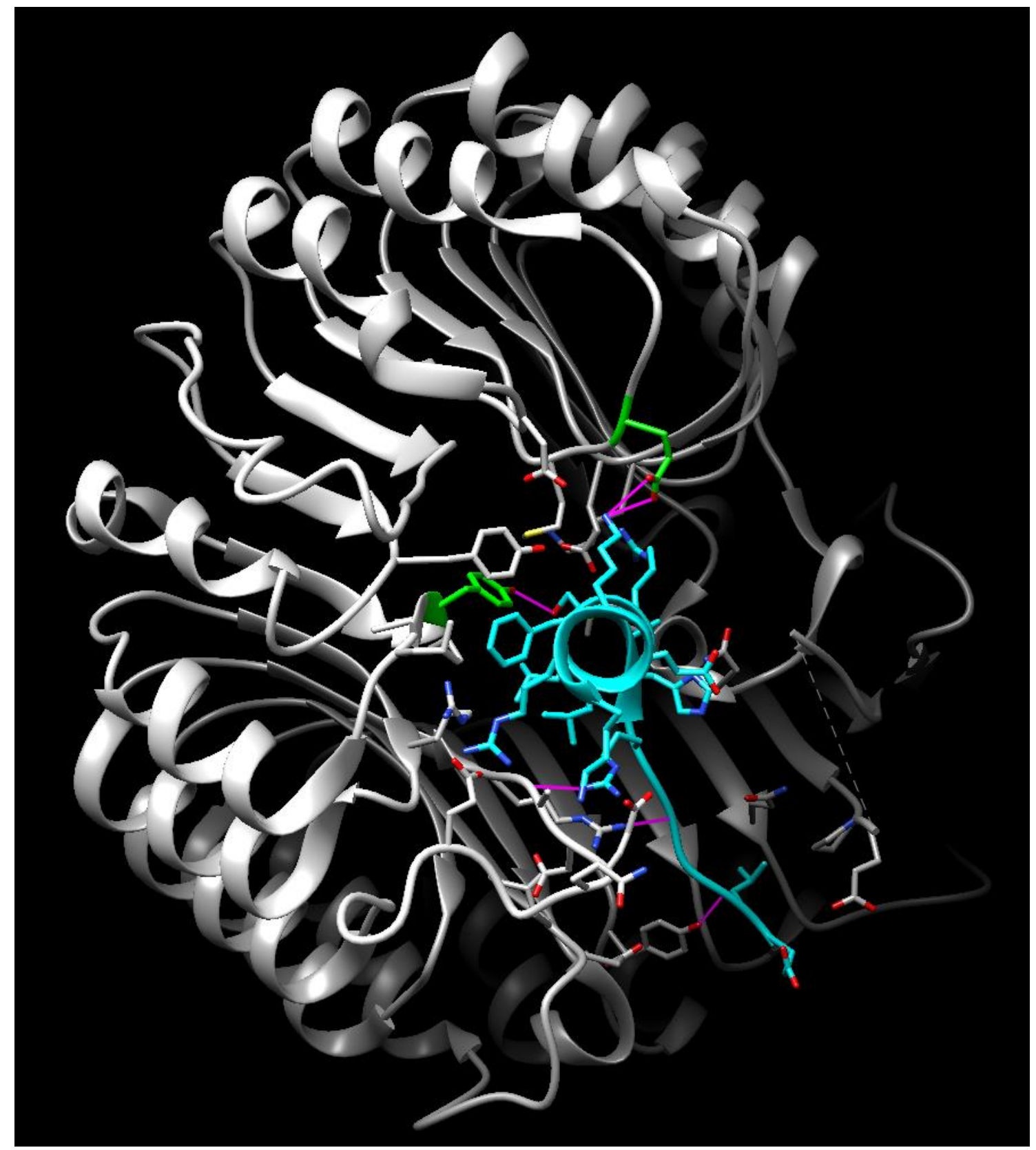

Figure S10. Top view of the JAZ1 docking helix bound to COI1.

Relevant amino acid residues of JAZ1 (cyan blue) and COI1 (grey) are shown in stick representation. Side chains of Glu203 and Tyr302 in COI1 are shown in green. Hydrogen bonds between the docking helix and COI1 are shown in magenta. 


\section{Figure S11}
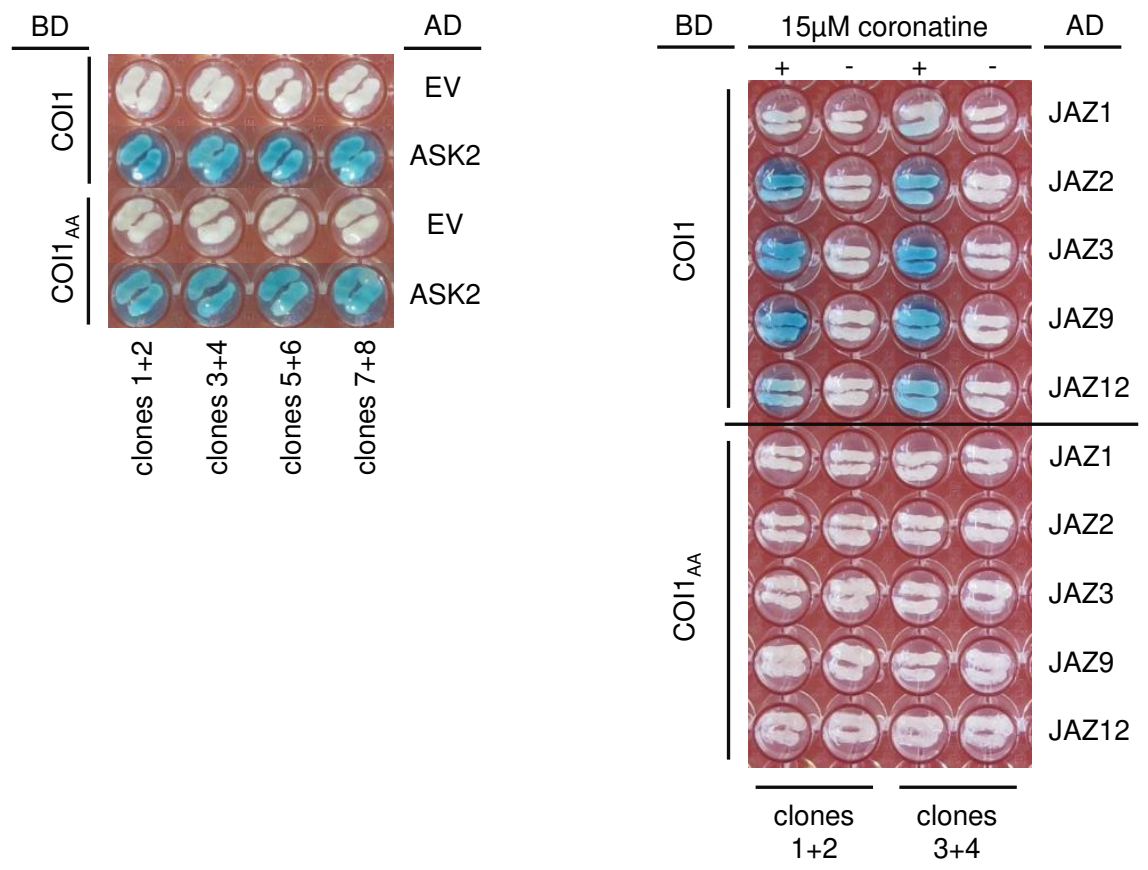

Figure S11. Coronatine-induced interaction of mutant $\mathrm{COI1}_{\mathrm{AA}}$ with JAZs is impaired.

Yeast strains co-expressing hybrid proteins composed of the LexA DNA binding domain (BD) fused to $\mathrm{COI1}_{\mathrm{WT}}$ and $\mathrm{CO}_{\mathrm{AA}}$ proteins, and the B42 activation domain (AD) fused to different JAZs or ASK2, or without fusion protein as empty vector control (EV), were streaked on media supplemented with X-Gal. Coronatine $(15 \mu \mathrm{M})$ was added to the media as indicated. As controls (-) the same volume of the solvent $\left(\mathrm{H}_{2} \mathrm{O}\right)$ was added. Blue color indicates protein interaction. 
Figure S12

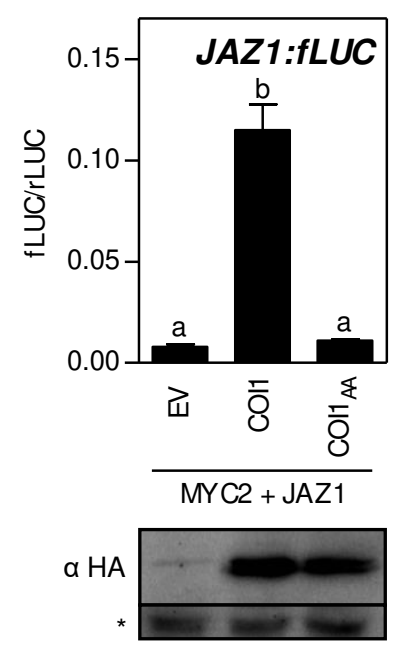

Figure S12. $\mathrm{COI1}_{\mathrm{AA}}$ is Impaired in mediating induction of the JAZ1 promoter in protoplasts.

Upper panel: Mesophyll protoplasts from the Arabidopsis thaliana coi1-t mutant were co-transfected with a reporter construct expressing firefly LUCIFERASE under control of the COI1-dependent Arabidopsis thaliana JAZ1 promoter (JAZ1:fLUC) $(5 \mu \mathrm{g})$ and plasmids enabling constitutive expression of MYC2 $(1.5 \mu \mathrm{g})$, JAZ1 $(5 \mu \mathrm{g})$ and HA-tagged COI1 or COI1 ${ }_{\mathrm{AA}}$ or empty vector (EV) $(5 \mu \mathrm{g})$. Firefly luciferase (fLUC) activities were normalized to Renilla luciferase (rLUC) activities. Values represent means \pm SEM of four independently transformed batches of protoplasts. For statistical analysis, a oneway ANOVA was performed followed by Tukey's multiple comparison test; lowercase letters denote significant differences between samples $(p<0.05)$. Lower panel: Expression of HA-COl1 proteins was assessed by Western blot analysis. ${ }^{*}$ depicts an unspecific band shown as loading control. 


\section{Figure $\mathrm{S} 13$}
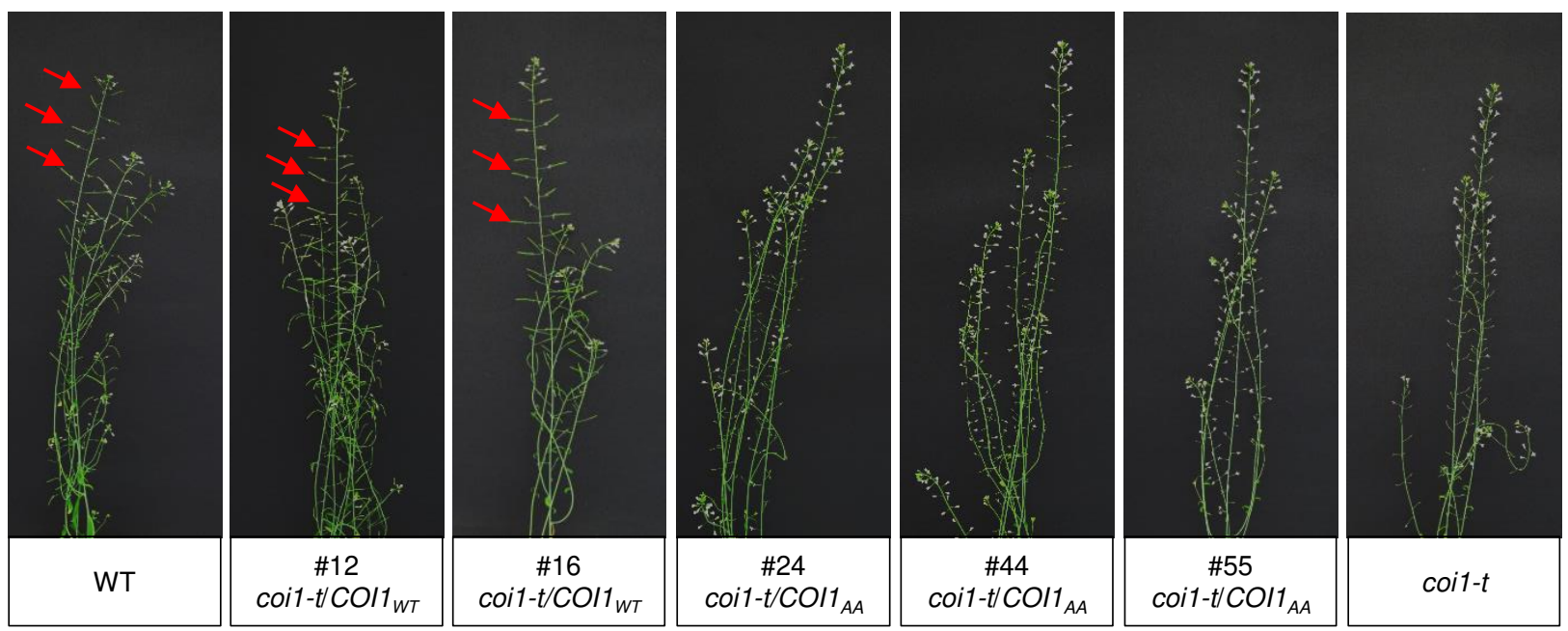

Figure S13. coi1-t plants expressing $\mathrm{COI}_{\mathrm{AA}}$ remain sterile.

Assessment of seed pod production in eight-week-old wild-type plants, two COl1 ${ }_{W T}$ complementation lines in the coi1- $t$ background, three $\mathrm{CO}{ }_{A A}$ complementation lines in the coi1- $t$ background and coi1- $t$ plants. Plants are from the T2 generation and are homozygous for the coi1-t allele and carry at least one copy of transgenic $\mathrm{COI1}_{W T}$ or $\mathrm{COI1}_{A A^{*}}$. Three to four plants from each line were monitored for seed pod production. Red arrows point at seed pods. 
Figure S14
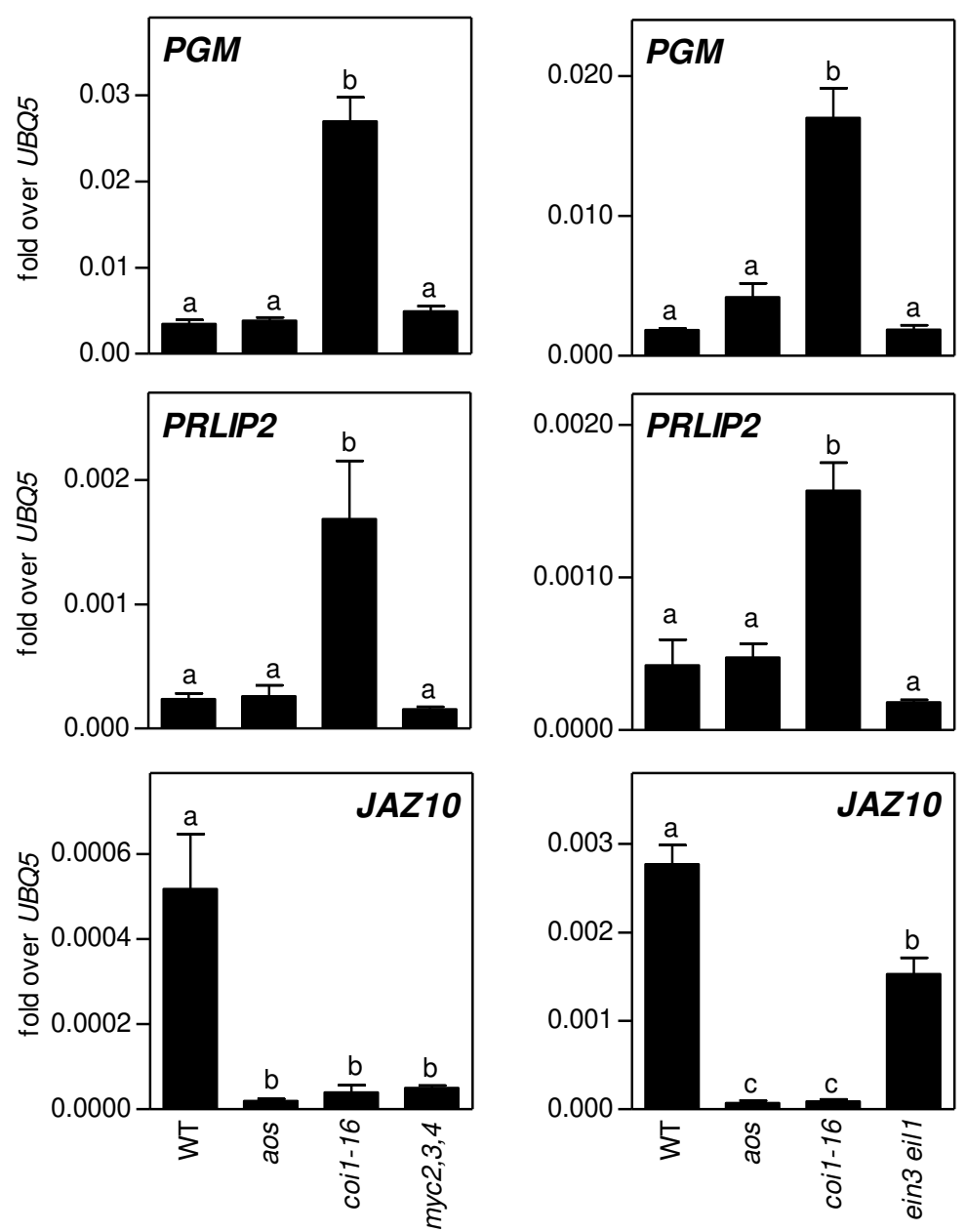

Figure S14. Col1-mediated repression of PGM and PRLIP2 does not involve known JAZinteracting transcription factors.

PGM, PRLIP2, and JAZ10 transcript levels, measured by qRT-PCR. RNA was extracted from mocktreated roots of wild-type, aos, coi1-16, myc 2,3,4 and ein3 eil1 seedlings grown on $1 / 2$ MS plates with subsequent cultivation on agarose in the absence of any added nutrients. Bars are means \pm SEM of three to five replicates with 30 roots per replicate. For statistical analysis, a one-way ANOVA was performed followed by Tukey's multiple comparison test; lowercase letters denote significant differences between samples $(p<0.05)$. 


\section{Supporting Tables}

Table S2: Primers for genotyping

\begin{tabular}{|c|c|c|}
\hline & Primer ID & Sequence 5'-3' \\
\hline \multirow[t]{2}{*}{ aos } & aos-fwd & AATCGTAGGACCAATCAAAGACCG \\
\hline & aos-rev & CAGATCCTTCTTCGCTCTACCGTA \\
\hline \multirow[t]{2}{*}{ bar } & BAR-fwd & GGTCTGCACCATCGTCAACCAC \\
\hline & BAR-rev & CAGCTGCCAGAAACCCACGTC \\
\hline \multirow[t]{3}{*}{ coi1-1 } & coi1-1 up & GTAATCGGAGATAGGGGTCTAGAGG \\
\hline & coi1-1 low & TGTACCCACAAGTATCTCAGTGAAGG \\
\hline & & Subsequent digestion with Mva1296I \\
\hline \multirow[t]{2}{*}{ coi1-16 } & coi1-16 fwd (Gutierrez et al., 2012) & AACTTCTACATGACGGAGTTTGC \\
\hline & coi1-16 rev (Gutierrez et al., 2012) & $\begin{array}{l}\text { GGAGCCACCACAAAATTCTTCIA (dCAPS primer } \\
\text { introducing an Xbal cleavage site into the } \\
\text { wildtype PCR product) }\end{array}$ \\
\hline \multirow[t]{3}{*}{ coi1-t } & COI1gen-1936fwd & CATCTTCTGGCTTTTCTGAAACAGCTG \\
\hline & COI1gen1115rev & CACCAATTTCATTAAGGACAAAAAGTATCCAC \\
\hline & LBb1 & GCGTGGACCGCTTGCTGCAACT \\
\hline \multirow{2}{*}{$\begin{array}{l}\mathrm{EV} \\
(H A-C O I 1)\end{array}$} & $\begin{array}{l}\text { pB2GW7-fwd (HA-COI1, empty } \\
\text { vector=pB2noHA, 35Sprom) }\end{array}$ & CACAATCCCACTATCCTTCGCA \\
\hline & $\begin{array}{l}\text { pB2GW7-rev (HA-COI1, empty } \\
\text { vector=pB2noHA, 35Sterm) }\end{array}$ & CATGAGCGAAACCCTATAAGAACC \\
\hline \multirow[t]{2}{*}{ med25 } & SALK_059316.56.00LP (pft1-3) & CATGGCGACGATCGAGTTGACCAAAGAAG \\
\hline & SALK_059316.56.00_RP (pft1-3) & CCTGACTTTGCATCAGGCAATATGTTGGC \\
\hline \multirow[t]{3}{*}{ sid2-2 } & sid2-2 fwd1 & TTCTTCATGCAGGGGAGGAG \\
\hline & sid2-2 fwd2 & CAACCACCTGGTGCACCAGC \\
\hline & sid2-2 rev & AAGCAAAATGTTTGAGTCAGCA \\
\hline \multirow{3}{*}{$\begin{array}{l}\text { COI1-HA- } \\
\text { Strep }\end{array}$} & ecoi-LPnew & TGGACCATATAAATTCATGCAGTCAACAAC \\
\hline & ecoi-RPnew & CTGCAGTGTGTAACGATGCTCAAAAGTC \\
\hline & LBb1.3 & ATTTTGCCGATTTCGGAAC \\
\hline
\end{tabular}


Table S3: Primers for qRT-PCR analysis

\begin{tabular}{|l|l|}
\hline Primer ID & Sequence 5'-3' $^{\prime}$ \\
\hline COI1-HA-Strep-RT fwd & AGTCCTGAAGGAGCCAATAGACCC \\
\hline COI1-HA-Strep-RT rev & TGAGACCAAGCGTAATCTGGAAC \\
\hline ICS1 & QuantiTect QT00893473 (Qiagen) \\
\hline JAZ10 & QuantiTect QT00828401 (Qiagen) \\
\hline PGM & QuantiTect QT00795879 (Qiagen) \\
\hline PRLIP2 & QuantiTect QT01833671 (Qiagen) \\
\hline SARD1 fwd RT & TCAAGGCGTTGTGGTTTGTG \\
\hline SARD1 rev RT & CGTCAACGACGGATAGTTTC \\
\hline UBQ5 fwd RT & GACGCTTCATCTCGTCC \\
\hline UBQ5 rev RT & GTAAACGTAGGTGAGTCCA \\
\hline VSP2 fwd RT & CAAACTAAACAATAAACCATACCATAA \\
\hline VSP2 rev RT & GCCAAGAGCAAGAGAAGTGA \\
\hline
\end{tabular}

Table S4: Primers for cloning

\begin{tabular}{|l|l|l|}
\hline & Primer ID & Sequence 5'-3' \\
\hline P1 & COI1GW-fwd & $\begin{array}{l}\text { GGGGACAAGTTTGTACAAAAAAGCAGGCTCCATGGAGGATCCTGAT } \\
\text { ATCAAGAGG }\end{array}$ \\
\hline P2 & COI1GW-rev & $\begin{array}{l}\text { GGGGACCACTTTGTACAAGAAAGCTGGGTCTCATATTGGCTCCTTCA } \\
\text { GGACTC }\end{array}$ \\
\hline P4 & COI1gGW-fwd & $\begin{array}{l}\text { GGGGACAAGTTTGTACAAAAAAGCAGGCTATTCCTCCTCGAGTGCAT } \\
\text { CATC }\end{array}$ \\
\hline P5 & coi1out-fwd & $\begin{array}{l}\text { GGGGACCACTTTGTACAAGAAAGCTGGGTCTATTGGCTCCTTCAGGA } \\
\text { CTCTAACAG }\end{array}$ \\
\hline
\end{tabular}


III. Article 1

\begin{tabular}{|l|l|l|}
\hline P6 & coi1E203-rev & ATTTTGGCAAAAGCCGTCATGTAGAAGTTTAAAACCTCAAG \\
\hline P7 & coi1E203-fwd & CATGACGGCTTTTGCCAAAATCAGTCCCAAAG \\
\hline P8 & coi1Y302-rev & GCAATGCAGCAAGCAAATCCAGCTTTCGG \\
\hline P9 & coi1Y302-fwd & GCTGGATTTGCTTGCTGCATTGCTAGAAACTGAAGACC \\
\hline P10 & coi1out-rev & GGATGCTCCATCTCTCTTATCTCTCC \\
\hline
\end{tabular}




\section{Supporting Experimental Procedures}

\section{Methods S1. RNAseq analysis}

For RNAseq analysis, 33 (coi1-t and $\mathrm{WT}_{\text {coit-t }}$ ) or 36 (aos and $\mathrm{WT}_{\text {aos }}$ ) single homozygous roots were combined for one replicate; replicates per genotype and treatment (two for coi1-t mock) were obtained from three independent infection experiments. RNA was extracted using the Trizol method (Chomczynski and Mackey, $1995)$ and RNA quality was controlled with an AGILENT BIOANALYZER 2100. Singleend 50-bp raw reads from mRNA sequencing were generated with the Illumina HiSeq 2000 platform and sequence images were transformed with the Illumina BaseCaller software to BCL files, which were subsequently demultiplexed to FASTQ files with CASAVA (v1.8.2). Using a Galaxy platform (Afgan et al., 2018), mapping of reads to the Arabidopsis thaliana genome reference sequence (TAIR10 release-39, $\mathrm{ftp}: / / \mathrm{ftp}$.ensemblgenomes.org/pub/plants/release-39) was carried out with RNA STAR (Galaxy version 2.5.2b-2 (Afgan et al., 2018)) and aligned reads were quantified using HTSeq-count (Galaxy version 0.9.1 (Anders et al., 2015)). Normalization and differential expression analysis was performed with DESeq2 (Galaxy version 2.11.40.6+galaxy1 (Love et al., 2014)) to obtain log2-fold changes and adjusted $p$ values (Benjamini-Hochberg-corrected). The agriGO v2.0 program was used for the functional classification of differentially expressed genes (Tian et al., 2017).

\section{Methods S2. Quantitative reverse transcription (qRT)-PCR and Western blot analysis}

Total RNA from frozen ground plant material was extracted with Trizol (Chomczynski and Mackey,1995). cDNA was synthesized from $1 \mu \mathrm{g}$ of total RNA. First, RNA was treated with $1 \cup$ DNase (Thermo Scientific, Vilnius, Lithuania) in $1 \times$ DNase I-Buffer with $\mathrm{MgCl}_{2}$ (Thermo Scientific, Vilnius, Lithuania) in a total volume of $10 \mu \mathrm{L}$. The mixture was incubated at $37^{\circ} \mathrm{C}$ for $30 \mathrm{~min}$ and the reaction was stopped by the addition of $1 \mu \mathrm{L}$ $25 \mathrm{mM}$ EDTA and further $10 \mathrm{~min}$ incubation at $65^{\circ} \mathrm{C}$. Next, 20 pmol of oligo(dT) (20mer and water were added to a total volume of $12.2 \mu \mathrm{L}$ and the mixture was incubated at $70^{\circ} \mathrm{C}$ for $10 \mathrm{~min}$. Finally, cDNA synthesis was completed by adding $20 \mathrm{pmol}$ deoxynucleotide triphosphate, $4 \mu \mathrm{L}$ of $5 \times \mathrm{RT}$ reaction buffer (Thermo Scientific, Vilnius, Lithuania), $60 \mathrm{U}$ of RevAid H-Minus Reverse Transcriptase (Thermo Scientific, Vilnius, 
Lithuania), topping up to $20 \mu \mathrm{L}$ total volume with water and incubating the mixture at $42^{\circ} \mathrm{C}$ for $70 \mathrm{~min}$. The reaction was stopped by incubation at $70^{\circ} \mathrm{C}$ for $10 \mathrm{~min}$. qRT-PCR analysis set up was as described (Fode et al., 2008) with SYBR Green from Lonza (Rockland, ME, USA). PCR consisted of a $90 \mathrm{~s}$ denaturation step at $95^{\circ} \mathrm{C}$ followed by 39 cycles of $20 \mathrm{~s}$ at $95^{\circ} \mathrm{C}, 20 \mathrm{~s}$ at $55^{\circ} \mathrm{C}$, and $40 \mathrm{~s}$ at $72^{\circ} \mathrm{C}$. Calculations were done according to the $2^{-\triangle C T}$ method (Livak and Schmittgen, 2001) using the UBQ5 (AT3G62250) transcript as a reference (Kesarwani et al., 2007). Primers serving to amplify and quantify transcript levels are listed in Table S3.

Expression of HA-tagged proteins in stably transformed plants was monitored by Western blot analysis. Protein extracts were prepared in $250 \mu \mathrm{l}$ extraction buffer (4 M urea, $16.6 \%$ glycerol, $5 \%$ SDS, $5 \% \beta$-mercaptoethanol) per $100 \mathrm{mg}$ plant material. Protein concentrations were determined using the Pierce $660 \mathrm{~nm}$ assay kit (Thermo Scientific, Rockford, IL USA). $50 \mu \mathrm{g}$ were loaded onto a $10 \%$ SDS gel. Proteins were detected using the $\alpha \mathrm{HA}$-antibody (Abcam, Cambridge, United Kingdom) and Super Signal $^{\mathrm{TM}}$ West Femto Maximum Sensitivity Substrate (Thermo Scientific, Rockford, IL USA).

\section{Methods S3. Construction of recombinant plasmids and generation of transgenic plants}

The GATEWAY technology (Invitrogen, Karlsruhe, Germany) was used to generate recombinant plasmids. The COl1 coding region was amplified from cDNA using primers that add GATEWAY recombination sites (P1-P2, Table S4) and inserted into pDONR201. The COI1 insert was subsequently recombined into pB2HAGW7. pB2HAGW7 originates from the binary vector pB2GW7.0 (http://www.psb.ugent.be/gateway/), but contains the expression cassette of $\mathrm{pE}-35 \mathrm{~S}$ HA-GW7 (Weiste et al., 2007). After confirming the sequence, the construct pB2-HACOI1 and the empty vector were first introduced into coi1-16 using Agrobacterium tumefaciens-mediated gene transfer (Clough and Bent, 1998). Plants expressing HACOI1 were identified by Western blot analysis using the $\alpha \mathrm{HA}$ antiserum. Later, the coi1-16 allele was replaced by the coi1- $t$ allele by fertilization of coi1- $t$ with pollen derived from 35S:HA-COI1 expressing coi1-16 plants. Plants homozygous for the transgene and the coi1- $t$ allele were used for further analysis. The plants transformed with the empty vector were maintained as a heterozygous population with respect to 
the coi1-t allele and had to be genotyped before analysis (see Table S2 for primers used for genotyping).

To create the C-terminally 3xHA-Strepll tagged genomic COl1 (CO/1g) constructs, the genomic COI1 region comprising 2287 bps upstream of the annotated transcriptional start site and the last amino acid of the coding region was amplified from Arabidopsis DNA (P3-P4, Table S4) and inserted into pDONR207. Generation of CO/1gAA was achieved by amplification of three fragments using primer pairs P5/P6, P7/P8 and P9/P10 with pDONR207/COl1g as a template. Primers P6 and P7 served to introduce the E203A mutation, while primers P8 and P9 served to introduce the $\mathrm{Y} 302 \mathrm{~A}$ mutation. The resulting three fragments served as templates for overlapping PCR with primers P5 and P10. The fragment was cut with Hindlll and EcoRI and ligated into the pDONR207-COl1g, also cut with HindllI and EcoRI. This step yielded pDONR207COl1gaA. Wild-type and mutant COl1g sequences were inserted into the destination vector pB-GW-HAS7 using the LR recombination reaction. pB-GW-HAS7 is a pB-GW derivative that carries an $3 \times \mathrm{HA}$ and a Strep tag downstream of the Gateway cassette. The sequence of the vector is given in Appendix 1. The resulting plasmids pB-COl1gHAS7 and pB-COl1gAA-HAS7 were introduced into heterozygous coi1-t plants which had been selected from the segregating population by pre-growth on MS medium containing $50 \mu \mathrm{M}$ MeJA (Reymond et al., 2000) to discard homozygous coi1-t plants and subsequent genotyping to discard wild-type plants. BASTA-selected plants were genotyped and plants heterozygous for coi1- $t$ were further characterized by Western blot analysis of leaf material using an $\alpha \mathrm{HA}$ antiserum. Plants expressing comparable amounts of wild-type and mutant COI1 were chosen for further analysis. Since plants were not homozygous with respect to the coi1-t allele and the transgene, they were genotyped directly before the experiment. After identifying homozygous coi1-t and $\mathrm{WT}_{\text {coit-t }}$ plants (Table S2), the selected plants underwent another round of genotyping. Homozygous coi1-t plants underwent PCR with ecoi-LPnew, ecoi-RPnew and LBb1.3 primers. A pattern of WT and homozygous bands together in this second PCR indicated at least one copy of transgenic $\mathrm{CO} 11_{\mathrm{AA}} / \mathrm{COI} 1 \mathrm{wT}$. A homozygous mutant band pattern alone meant an absence of transgenic $\mathrm{CO} 11_{\mathrm{AA}} / \mathrm{COI} 1_{\mathrm{WT}}$. The latter plants were

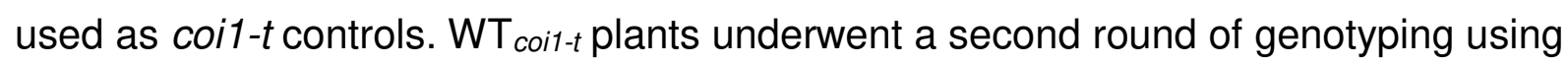
bar primers (BASTA resistance) to identify plants not carrying the COl1 AA or COI1WT construct, which were used as WT controls. Additionally, qRT-PCR using COI1-HA- 
Strep-RT fwd and COI1-HA-Strep-RT rev primers was used for all plants to confirm the presence or absence of COl1 $1_{\mathrm{AA}}$ or COl1wT.

\section{Methods S4. Fungal culture}

Verticillium longisporum VI43 was grown in Potato Dextrose Medium with $0.5 \mathrm{mg} / \mathrm{L}$ Cefotaxim for 14 days at $21^{\circ} \mathrm{C}, 90 \mathrm{rpm}$, in the dark. Spores were harvested by straining through a filter (Nucleo Bond Folded filters, Macherey-Nagel, Düren, Germany). Spores were washed in sterile tap water, the spore concentration determined with a hemocytometer and finally spores were diluted to $1 \times 10^{6}$ spores $/ \mathrm{mL}$ for sand-soil infections or $1 \times 10^{5}$ spores $/ \mathrm{mL}$ for plate infections.

\section{Methods S5. Leaf area measurement}

Photographs of individual plants were taken and the surface area of the whole rosette was determined using 'BlattFlaeche' Software (Datinf $\mathrm{GmbH}$, Tübingen, Germany) (Ralhan et al., 2012).

\section{Methods S6. Assessment of COl1 activity in transiently transformed protoplasts}

Construction of plasmids and transient assays were performed essentially as described ( $\mathrm{Li}$ et al., 2019b). JAZ sequences were amplified and inserted into pDONR207 and subsequently recombined into the destination vector UBQ10pro:HAGW.

\section{Methods S7. Yeast two-hybrid analysis}

COI1, ASK2 and JAZ sequences were transferred to the GATEWAY-compatible vectors for the LexA yeast two-hybrid system pGILDA-GW and pB42AD-GW described earlier (Li et al., 2019a). Yeast two-hybrid experiments were performed as described previously (Zhang et al., 2015). Plasmids were transformed into yeast strain Saccharomyces cerevisiae EGY48 (Estojak et al., 1995) harboring the LexA reporter plasmid p8opLacZ (pSH18-34, Invitrogen, Thermo Fisher Scientific, Dreieich, 
Germany) using the PEG-LiAc method (Gietz et al., 1992). Transformants were selected on Synthetic Defined (SD) medium supplemented with glucose and -Ura/His/-Trp drop-out solution. To assess the interaction, preselected yeast transformants were streaked onto SD medium supplemented with galactose, raffinose, -Ura/-His/Trp drop-out solution, containing $80 \mu \mathrm{g} / \mathrm{ml} \mathrm{X-Gal.} \mathrm{To} \mathrm{trigger} \mathrm{COI1-JAZ} \mathrm{interactions,} 15$ $\mu \mathrm{M}$ coronatine was added.

\section{REFERENCES for Experimental Procedures}

Afgan, E., Baker, D., Batut, B., van den Beek, M., Bouvier, D., Cech, M., Chilton, J., Clements, D., Coraor, N., Gruning, B.A., Guerler, A., Hillman-Jackson, J., Hiltemann, S., Jalili, V., Rasche, H., Soranzo, N., Goecks, J., Taylor, J., Nekrutenko, A. and Blankenberg, D. (2018) The Galaxy platform for accessible, reproducible and collaborative biomedical analyses: 2018 update. Nucleic Acids Res, 46, W537-W544.

Anders, S., Pyl, P.T. and Huber, W. (2015) HTSeq--a Python framework to work with high-throughput sequencing data. Bioinformatics, 31, 166-169.

Chomczynski, P. and Mackey, K. (1995) Short technical reports. Modification of the TRI reagent procedure for isolation of RNA from polysaccharide- and proteoglycan-rich sources. Biotechniques, 19, 942-945.

Clough, S.J. and Bent, A.F. (1998) Floral dip: a simplified method for Agrobacteriummediated transformation of Arabodopsis thaliana. Plant J, 16, 735-743.

Estojak, J., Brent, R. and Golemis, E.A. (1995) Correlation of two-hybrid affinity data with in vitro measurements. Mol Cell Biol, 15, 5820-5829.

Fode, B., Siemsen, T., Thurow, C., Weigel, R. and Gatz, C. (2008) The Arabidopsis GRAS Protein SCL14 Interacts with Class II TGA Transcription Factors and Is Essential for the Activation of Stress-Inducible Promoters. Plant Cell, 20, 31223135.

Gietz, D., St Jean, A., Woods, R.A. and Schiestl, R.H. (1992) Improved method for high efficiency transformation of intact yeast cells. Nucleic Acids Res, 20, 1425.

Gutierrez, L., Mongelard, G., Flokova, K., Pacurar, D.I., Novak, O., Staswick, P., Kowalczyk, M., Pacurar, M., Demailly, H., Geiss, G. and Bellini, C. (2012) Auxin controls Arabidopsis adventitious root initiation by regulating jasmonic acid homeostasis. Plant Cell, 24, 2515-2527. 
Li, N., Muthreich, M., Huang, L.J., Thurow, C., Sun, T., Zhang, Y. and Gatz, C. (2019a) TGACG-BINDING FACTORs (TGAs) and TGA-interacting CC-type glutaredoxins modulate hyponastic growth in Arabidopsis thaliana. New Phytolol, 221, 1906-1918.

Li, N., Uhrig, J.F., Thurow, C., Huang, L.J. and Gatz, C. (2019b) Reconstitution of the Jasmonate Signaling Pathway in Plant Protoplasts. Cells, 8, 1532.

Livak, K.J. and Schmittgen, T.D. (2001) Analysis of relative gene expression data using real-time quantitative PCR and the 2(-Delta Delta C(T)) Method. Methods (San Diego, Calif), 25, 402-408.

Love, M.I., Huber, W. and Anders, S. (2014) Moderated estimation of fold change and dispersion for RNA-seq data with DESeq2. Genome Biol, 15, 550.

Tian, T., Liu, Y., Yan, H., You, Q., Yi, X., Du, Z., Xu, W. and Su, Z. (2017) agriGO v2.0: a GO analysis toolkit for the agricultural community, 2017 update. Nucleic Acids Res, 45, W122-W129.

Weiste, C., Iven, T., Fischer, U., Onate-Sanchez, L. and Droge-Laser, W. (2007) In planta ORFeome analysis by large-scale over-expression of GATEWAYcompatible cDNA clones: screening of ERF transcription factors involved in abiotic stress defense. Plant J, 52, 382-390. 


\section{Appendix 1, Sequence of pB-GW-HAS7}

CGACGTCGCATGCTCCCGGCCGCCATGGCCGCGGGATATCACAAGTTTGTACAAAAAAGCTGAACGAGAAAC GTAAAATGATATAAATATCAATATATTAAATTAGATTTTGCATAAAAAACAGACTACATAATACTGTAAAACAC AACATATCCAGTCACTATGGCGGCCGCATTAGGCACCCCAGGCTTTACACTTTATGCTTCCGGCTCGTATAATG TGTGGATTTTGAGTTAGGATCCGTCGAGATTTTCAGGAGCTAAGGAAGCTAAAATGGAGAAAAAAATCACTG GATATACCACCGTTGATATATCCCAATGGCATCGTAAAGAACATTTTGAGGCATTTCAGTCAGTTGCTCAATGT ACCTATAACCAGACCGTTCAGCTGGATATTACGGCCTTTTTAAAGACCGTAAAGAAAAATAAGCACAAGTTTTA TCCGGCCTTTATTCACATTCTTGCCCGCCTGATGAATGCTCATCCGGAATTCCGTATGGCAATGAAAGACGGTG AGCTGGTGATATGGGATAGTGTTCACCCTTGTTACACCGTTTTCCATGAGCAAACTGAAACGTTTTCATCGCTCT GGAGTGAATACCACGACGATTTCCGGCAGTTTCTACACATATATTCGCAAGATGTGGCGTGTTACGGTGAAAA CCTGGCCTATTTCCCTAAAGGGTTTATTGAGAATATGTTTTTCGTCTCAGCCAATCCCTGGGTGAGTTTCACCAG TTTTGATTTAAACGTGGCCAATATGGACAACTTCTTCGCCCCCGTTTTCACCATGGGCAAATATTATACGCAAGG CGACAAGGTGCTGATGCCGCTGGCGATTCAGGTTCATCATGCCGTTTGTGATGGCTTCCATGTCGGCAGAATG CTTAATGAATTACAACAGTACTGCGATGAGTGGCAGGGCGGGGCGTAAAGATCTGGATCCGGCTTACTAAAA GCCAGATAACAGTATGCGTATTTGCGCGCTGATTTTTGCGGTATAAGAATATATACTGATATGTATACCCGAAG TATGTCAAAAAGAGGTATGCTATGAAGCAGCGTATTACAGTGACAGTTGACAGCGACAGCTATCAGTTGCTCA AGGCATATATGATGTCAATATCTCCGGTCTGGTAAGCACAACCATGCAGAATGAAGCCCGTCGTCTGCGTGCC GAACGCTGGAAAGCGGAAAATCAGGAAGGGATGGCTGAGGTCGCCCGGTTTATTGAAATGAACGGCTCTTTT GCTGACGAGAACAGGGGCTGGTGAAATGCAGTTTAAGGTTTACACCTATAAAAGAGAGAGCCGTTATCGTCT GTTTGTGGATGTACAGAGTGATATTATTGACACGCCCGGGCGACGGATGGTGATCCCCCTGGCCAGTGCACGT CTGCTGTCAGATAAAGTCTCCCGTGAACTTTACCCGGTGGTGCATATCGGGGATGAAAGCTGGCGCATGATGA CCACCGATATGGCCAGTGTGCCGGTCTCCGTTATCGGGGAAGAAGTGGCTGATCTCAGCCACCGCGAAAATGA CATCAAAAACGCCATTAACCTGATGTTCTGGGGAATATAAATGTCAGGCTCCCTTATACACAGCCAGTCTGCAG GTCGACCATAGTGACTGGATATGTTGTGTTTTACAGTATTATGTAGTCTGTTTTTTATGCAAAATCTAATTTAAT ATATTGATATTTATATCATTTTACGTTTCTCGTTCAGCTTTCTTGTACAAAGTGGTTGATGGGTACCCATACGAT GTTCCTGACTATGCGGGCTATCCCTATGACGTCCCGGACTATGCAGGATCCTATCCATATGACGTTCCAGATTA CGCTTGGTCTCATCCTCAATTTGAAAAATAATCTAGAGTCCGCAAAAATCACCAGTCTCTCTCTACAAATCTATC TCTCTCTATTTTTCTCCAGAATAATGTGTGAGTAGTTCCCAGATAAGGGAATTAGGGTTCTTATAGGGTTTCGCT CATGTGTTGAGCATATAAGAAACCCTTAGTATGTATTTGTATTTGTAAAATACTTCTATCAATAAAATTTCTAATT CCTAAAACCAAAATCCAGTGACCGGGCGGCCGCCACCGCGGTGGAGGGGGATCAGATTGTCGTTTCCCGCCTT CAGTTTAAACTATCAGTGTTTGACAGGATATATTGGCGGGTAAACCTAAGAGAAAAGAGCGTTTATTAGAATA ATCGGATATTTAAAAGGGCGTGAAAAGGTTTATCCGTTCGTCCATTTGTATGTGCATGCCAACCACAGGGTTCC CCTCGGGATCAAAGTACTTTAAAGTACTTTAAAGTACTTTAAAGTACTTTGATCCAACCCCTCCGCTGCTATAGT GCAGTCGGCTTCTGACGTTCAGTGCAGCCGTCTTCTGAAAACGACATGTCGCACAAGTCCTAAGTTACGCGAC AGGCTGCCGCCCTGCCCTTTTCCTGGCGTTTTCTTGTCGCGTGTTTTAGTCGCATAAAGTAGAATACTTGCGACT AGAACCGGAGACATTACGCCATGAACAAGAGCGCCGCCGCTGGCCTGCTGGGCTATGCCCGCGTCAGCACCG ACGACCAGGACTTGACCAACCAACGGGCCGAACTGCACGCGGCCGGCTGCACCAAGCTGTTTTCCGAGAAGA TCACCGGCACCAGGCGCGACCGCCCGGAGCTGGCCAGGATGCTTGACCACCTACGCCCTGGCGACGTTGTGA CAGTGACCAGGCTAGACCGCCTGGCCCGCAGCACCCGCGACCTACTGGACATTGCCGAGCGCATCCAGGAGG CCGGCGCGGGCCTGCGTAGCCTGGCAGAGCCGTGGGCCGACACCACCACGCCGGCCGGCCGCATGGTGTTGA CCGTGTTCGCCGGCATTGCCGAGTTCGAGCGTTCCCTAATCATCGACCGCACCCGGAGCGGGCGCGAGGCCGC CAAGGCCCGAGGCGTGAAGTTTGGCCCCCGCCCTACCCTCACCCCGGCACAGATCGCGCACGCCCGCGAGCTG ATCGACCAGGAAGGCCGCACCGTGAAAGAGGCGGCTGCACTGCTTGGCGTGCATCGCTCGACCCTGTACCGC GCACTTGAGCGCAGCGAGGAAGTGACGCCCACCGAGGCCAGGCGGCGCGGTGCCTTCCGTGAGGACGCATT GACCGAGGCCGACGCCCTGGCGGCCGCCGAGAATGAACGCCAAGAGGAACAAGCATGAAACCGCACCAGGA 
CGGCCAGGACGAACCGTTTTTCATTACCGAAGAGATCGAGGCGGAGATGATCGCGGCCGGGTACGTGTTCGA GCCGCCCGCGCACGTCTCAACCGTGCGGCTGCATGAAATCCTGGCCGGTTTGTCTGATGCCAAGCTGGCGGCC TGGCCGGCCAGCTTGGCCGCTGAAGAAACCGAGCGCCGCCGTCTAAAAAGGTGATGTGTATTTGAGTAAAAC AGCTTGCGTCATGCGGTCGCTGCGTATATGATGCGATGAGTAAATAAACAAATACGCAAGGGGAACGCATGA AGGTTATCGCTGTACTTAACCAGAAAGGCGGGTCAGGCAAGACGACCATCGCAACCCATCTAGCCCGCGCCCT GCAACTCGCCGGGGCCGATGTTCTGTTAGTCGATTCCGATCCCCAGGGCAGTGCCCGCGATTGGGCGGCCGTG CGGGAAGATCAACCGCTAACCGTTGTCGGCATCGACCGCCCGACGATTGACCGCGACGTGAAGGCCATCGGC CGGCGCGACTTCGTAGTGATCGACGGAGCGCCCCAGGCGGCGGACTTGGCTGTGTCCGCGATCAAGGCAGCC GACTTCGTGCTGATTCCGGTGCAGCCAAGCCCTTACGACATATGGGCCACCGCCGACCTGGTGGAGCTGGTTA AGCAGCGCATTGAGGTCACGGATGGAAGGCTACAAGCGGCCTTTGTCGTGTCGCGGGCGATCAAAGGCACGC GCATCGGCGGTGAGGTTGCCGAGGCGCTGGCCGGGTACGAGCTGCCCATTCTTGAGTCCCGTATCACGCAGC GCGTGAGCTACCCAGGCACTGCCGCCGCCGGCACAACCGTTCTTGAATCAGAACCCGAGGGCGACGCTGCCC GCGAGGTCCAGGCGCTGGCCGCTGAAATTAAATCAAAACTCATTTGAGTTAATGAGGTAAAGAGAAAATGAG CAAAAGCACAAACACGCTAAGTGCCGGCCGTCCGAGCGCACGCAGCAGCAAGGCTGCAACGTTGGCCAGCCT GGCAGACACGCCAGCCATGAAGCGGGTCAACTTTCAGTTGCCGGCGGAGGATCACACCAAGCTGAAGATGTA CGCGGTACGCCAAGGCAAGACCATTACCGAGCTGCTATCTGAATACATCGCGCAGCTACCAGAGTAAATGAGC AAATGAATAAATGAGTAGATGAATTTTAGCGGCTAAAGGAGGCGGCATGGAAAATCAAGAACAACCAGGCAC CGACGCCGTGGAATGCCCCATGTGTGGAGGAACGGGCGGTTGGCCAGGCGTAAGCGGCTGGGTTGTCTGCC GGCCCTGCAATGGCACTGGAACCCCCAAGCCCGAGGAATCGGCGTGACGGTCGCAAACCATCCGGCCCGGTA CAAATCGGCGCGGCGCTGGGTGATGACCTGGTGGAGAAGTTGAAGGCCGCGCAGGCCGCCCAGCGGCAACG CATCGAGGCAGAAGCACGCCCCGGTGAATCGTGGCAAGCGGCCGCTGATCGAATCCGCAAAGAATCCCGGCA ACCGCCGGCAGCCGGTGCGCCGTCGATTAGGAAGCCGCCCAAGGGCGACGAGCAACCAGATTTTTTCGTTCC GATGCTCTATGACGTGGGCACCCGCGATAGTCGCAGCATCATGGACGTGGCCGTTTTCCGTCTGTCGAAGCGT GACCGACGAGCTGGCGAGGTGATCCGCTACGAGCTTCCAGACGGGCACGTAGAGGTTTCCGCAGGGCCGGCC GGCATGGCCAGTGTGTGGGATTACGACCTGGTACTGATGGCGGTTTCCCATCTAACCGAATCCATGAACCGAT ACCGGGAAGGGAAGGGAGACAAGCCCGGCCGCGTGTTCCGTCCACACGTTGCGGACGTACTCAAGTTCTGCC GGCGAGCCGATGGCGGAAAGCAGAAAGACGACCTGGTAGAAACCTGCATTCGGTTAAACACCACGCACGTTG CCATGCAGCGTACGAAGAAGGCCAAGAACGGCCGCCTGGTGACGGTATCCGAGGGTGAAGCCTTGATTAGCC GCTACAAGATCGTAAAGAGCGAAACCGGGCGGCCGGAGTACATCGAGATCGAGCTAGCTGATTGGATGTACC GCGAGATCACAGAAGGCAAGAACCCGGACGTGCTGACGGTTCACCCCGATTACTTTTTGATCGATCCCGGCAT CGGCCGTTTTCTCTACCGCCTGGCACGCCGCGCCGCAGGCAAGGCAGAAGCCAGATGGTTGTTCAAGACGATC TACGAACGCAGTGGCAGCGCCGGAGAGTTCAAGAAGTTCTGTTTCACCGTGCGCAAGCTGATCGGGTCAAAT GACCTGCCGGAGTACGATTTGAAGGAGGAGGCGGGGCAGGCTGGCCCGATCCTAGTCATGCGCTACCGCAAC CTGATCGAGGGCGAAGCATCCGCCGGTTCCTAATGTACGGAGCAGATGCTAGGGCAAATTGCCCTAGCAGGG GAAAAAGGTCGAAAAGGTCTCTTTCCTGTGGATAGCACGTACATTGGGAACCCAAAGCCGTACATTGGGAACC GGAACCCGTACATTGGGAACCCAAAGCCGTACATTGGGAACCGGTCACACATGTAAGTGACTGATATAAAAG AGAAAAAAGGCGATTTTTCCGCCTAAAACTCTTTAAAACTTATTAAAACTCTTAAAACCCGCCTGGCCTGTGCAT AACTGTCTGGCCAGCGCACAGCCGAAGAGCTGCAAAAAGCGCCTACCCTTCGGTCGCTGCGCTCCCTACGCCC CGCCGCTTCGCGTCGGCCTATCGCGGCCGCTGGCCGCTCAAAAATGGCTGGCCTACGGCCAGGCAATCTACCA GGGCGCGGACAAGCCGCGCCGTCGCCACTCGACCGCCGGCGCCCACATCAAGGCACCCTGCCTCGCGCGTTTC GGTGATGACGGTGAAAACCTCTGACACATGCAGCTCCCGGAGACGGTCACAGCTTGTCTGTAAGCGGATGCC GGGAGCAGACAAGCCCGTCAGGGCGCGTCAGCGGGTGTTGGCGGGTGTCGGGGCGCAGCCATGACCCAGTC ACGTAGCGATAGCGGAGTGTATACTGGCTTAACTATGCGGCATCAGAGCAGATTGTACTGAGAGTGCACCATA TGCGGTGTGAAATACCGCACAGATGCGTAAGGAGAAAATACCGCATCAGGCGCTCTTCCGCTTCCTCGCTCAC TGACTCGCTGCGCTCGGTCGTTCGGCTGCGGCGAGCGGTATCAGCTCACTCAAAGGCGGTAATACGGTTATCC ACAGAATCAGGGGATAACGCAGGAAAGAACATGTGAGCAAAAGGCCAGCAAAAGGCCAGGAACCGTAAAAA 
GGCCGCGTTGCTGGCGTTTTTCCATAGGCTCCGCCCCCCTGACGAGCATCACAAAAATCGACGCTCAAGTCAG AGGTGGCGAAACCCGACAGGACTATAAAGATACCAGGCGTTTCCCCCTGGAAGCTCCCTCGTGCGCTCTCCTG TTCCGACCCTGCCGCTTACCGGATACCTGTCCGCCTTTCTCCCTTCGGGAAGCGTGGCGCTTTCTCATAGCTCAC GCTGTAGGTATCTCAGTTCGGTGTAGGTCGTTCGCTCCAAGCTGGGCTGTGTGCACGAACCCCCCGTTCAGCCC GACCGCTGCGCCTTATCCGGTAACTATCGTCTTGAGTCCAACCCGGTAAGACACGACTTATCGCCACTGGCAGC AGCCACTGGTAACAGGATTAGCAGAGCGAGGTATGTAGGCGGTGCTACAGAGTTCTTGAAGTGGTGGCCTAA CTACGGCTACACTAGAAGGACAGTATTTGGTATCTGCGCTCTGCTGAAGCCAGTTACCTTCGGAAAAAGAGTT GGTAGCTCTTGATCCGGCAAACAAACCACCGCTGGTAGCGGTGGTTTTTTTGTTTGCAAGCAGCAGATTACGC GCAGAAAAAAAGGATCTCAAGAAGATCCTTTGATCTTTTCTACGGGGTCTGACGCTCAGTGGAACGAAAACTC ACGTTAAGGGATTTTGGTCATGCATGATATATCTCCCAATTTGTGTAGGGCTTATTATGCACGCTTAAAAATAAT AAAAGCAGACTTGACCTGATAGTTTGGCTGTGAGCAATTATGTGCTTAGTGCATCTAATCGCTTGAGTTAACGC CGGCGAAGCGGCGTCGGCTTGAACGAATTTCTAGCTAGACATTATTTGCCGACTACCTTGGTGATCTCGCCTTT CACGTAGTGGACAAATTCTTCCAACTGATCTGCGCGCGAGGCCAAGCGATCTTCTTCTTGTCCAAGATAAGCCT GTCTAGCTTCAAGTATGACGGGCTGATACTGGGCCGGCAGGCGCTCCATTGCCCAGTCGGCAGCGACATCCTT CGGCGCGATTTTGCCGGTTACTGCGCTGTACCAAATGCGGGACAACGTAAGCACTACATTTCGCTCATCGCCA GCCCAGTCGGGCGGCGAGTTCCATAGCGTTAAGGTTTCATTTAGCGCCTCAAATAGATCCTGTTCAGGAACCG GATCAAAGAGTTCCTCCGCCGCTGGACCTACCAAGGCAACGCTATGTTCTCTTGCTTTTGTCAGCAAGATAGCC AGATCAATGTCGATCGTGGCTGGCTCGAAGATACCTGCAAGAATGTCATTGCGCTGCCATTCTCCAAATTGCAG TTCGCGCTTAGCTGGATAACGCCACGGAATGATGTCGTCGTGCACAACAATGGTGACTTCTACAGCGCGGAGA ATCTCGCTCTCTCCAGGGGAAGCCGAAGTTTCCAAAAGGTCGTTGATCAAAGCTCGCCGCGTTGTTTCATCAAG CCTTACGGTCACCGTAACCAGCAAATCAATATCACTGTGTGGCTTCAGGCCGCCATCCACTGCGGAGCCGTACA AATGTACGGCCAGCAACGTCGGTTCGAGATGGCGCTCGATGACGCCAACTACCTCTGATAGTTGAGTCGATAC TTCGGCGATCACCGCTTCCCCCATGATGTTTAACTTTGTTTTAGGGCGACTGCCCTGCTGCGTAACATCGTTGCT GCTCCATAACATCAAACATCGACCCACGGCGTAACGCGCTTGCTGCTTGGATGCCCGAGGCATAGACTGTACC CCAAAAAAACATGTCATAACAAGAAGCCATGAAAACCGCCACTGCGCCGTTACCACCGCTGCGTTCGGTCAAG GTTCTGGACCAGTTGCGTGACGGCAGTTACGCTACTTGCATTACAGCTTACGAACCGAACGAGGCTTATGTCCA CTGGGTTCGTGCCCGAATTGATCACAGGCAGCAACGCTCTGTCATCGTTACAATCAACATGCTACCCTCCGCGA GATCATCCGTGTTTCAAACCCGGCAGCTTAGTTGCCGTTCTTCCGAATAGCATCGGTAACATGAGCAAAGTCTG CCGCCTTACAACGGCTCTCCCGCTGACGCCGTCCCGGACTGATGGGCTGCCTGTATCGAGTGGTGATTTTGTGC CGAGCTGCCGGTCGGGGAGCTGTTGGCTGGCTGGTGGCAGGATATATTGTGGTGTAAACAAATTGACGCTTA GACAACTTAATAACACATTGCGGACGTTTTTAATGTACTGAATTAACGCCGAATTGAATTATCAGCTTGCATGC CGGTCGATCTAGTAACATAGTAGATGACACCGCGCGCGATAATTTATCCTAGTTTGCGCGCTATATTTTGTTTTC TATCGCGTATTAAATGTATAATTGCGGGACTCTAATCATAAAAACCCATCTCATAAATAACGTCATGCATTACAT GTTAATTATTACATGCTTAACGTAATTCAACAGAAATTATATGATAATCATCGCAAGACCGGCAACAGGATTCA ATCTTAAGAAACTTTATTGCCAAATGTTTGAACGATCTGCTTGACTCTAGGGGTCATCAGATTTCGGTGACGGG CAGGACCGGACGGGGCGGCACCGGCAGGCTGAAGTCCAGCTGCCAGAAACCCACGTCATGCCAGTTCCCGTG CTTGAAGCCGGCCGCCCGCAGCATGCCGCGGGGGGCATATCCGAGCGCCTCGTGCATGCGCACGCTCGGGTC GTTGGGCAGCCCGATGACAGCGACCACGCTCTTGAAGCCCTGTGCCTCCAGGGACTTCAGCAGGTGGGTGTA GAGCGTGGAGCCCAGTCCCGTCCGCTGGTGGCGGGGGGAGACGTACACGGTCGACTCGGCCGTCCAGTCGTA GGCGTTGCGTGCCTTCCAGGGACCCGCGTAGGCGATGCCGGCGACCTCGCCGTCCACCTCGGCGACGAGCCA GGGATAGCGCTCCCGCAGACGGACGAGGTCGTCCGTCCACTCCTGCGGTTCCTGCGGCTCGGTACGGAAGTT GACCGTGCTTGTCTCGATGTAGTGGTTGACGATGGTGCAGACCGCCGGCATGTCCGCCTCGGTGGCACGGCG GATGTCGGCCGGGCGTCGTTCTGGGCTCATGGTAGATCCCCTCGATCGAGTTGAGAGTGAATATGAGACTCTA ATTGGATACCGAGGGGAATTTATGGAACGTCAGTGGAGCATTTTTGACAAGAAATATTTGCTAGCTGATAGTG ACCTTAGGCGACTTTTGAACGCGCAATAATGGTTTCTGACGTATGTGCTTAGCTCATTAAACTCCAGAAACCCG 
III. Article 1

CGGCTCAGTGGCTCCTTCAACGTTGCGGTTCTGTCAGTTCCAAACGTAAAACGGCTTGTCCCGCGTCATCGGCG GGGGTCATAACGTGACTCCCTTAATTCTCATGTATGATAATTCGAGGGTACCCGGGGATCCTCTAGAGGGCC 


\title{
IV. Article 2
}

Induction of salicylic acid-related defence genes in Arabidopsis roots upon infection with Verticillium longisporum requires transcription factor SARD1 and the inactivation of COl1-mediated repression

\author{
Louisa Ulrich, Johanna Schmitz, Corinna Thurow and Christiane Gatz ${ }^{1}$
}

Department of Plant Molecular Biology and Physiology, Albrecht-von-Haller Institute for Plant Sciences, University of Göttingen, Julia-Lermontowa-Weg 3, 37077 Göttingen, Germany

1'Corresponding author: cgatz@gwdg.de

This manuscript is ready for submission.

\section{Detailed contributions by the PhD candidate:}

Louisa Ulrich prepared, performed and analysed all experiments presented in this manuscript with exception of the RNA-seq experiment, the Motif Mapper analysis and the SA measurements. Louisa Ulrich generated the coi1-t sard1-1 cbp60g-1 lines and characterised the SARD1 overexpression lines. Louisa Ulrich created all Figures with the exception of Figure $1 \mathrm{a}$ and wrote a complete first draft of the manuscript.

Table 1 (RNA-seq data) will be deposited at the department of Plant Molecular Biology and Physiology, Georg-August University Göttingen, until the manuscript is published. For access, please contact Prof. C. Gatz (cgatz@gwdg.de). 


\title{
Induction of salicylic acid-related defence genes in Arabidopsis roots upon infection with Verticillium longisporum requires transcription factor SARD1 and the inactivation of COl1-mediated repression
}

Louisa Ulrich, Johanna Schmitz, Corinna Thurow and Christiane Gatz ${ }^{1}$

Department of Plant Molecular Biology and Physiology, Albrecht-von-Haller Institute for Plant Sciences, University of Göttingen, Julia-Lermontowa-Weg 3, 37077 Göttingen, Germany

1Corresponding author: cgatz@gwdg.de

Running title: The repressive effect of COI1 on SA-related defence genes in roots is overcome by $V$. longisporum infections in the absence of JA.

\begin{abstract}
Verticillium longisporum is a soil-borne fungal pathogen causing vascular disease predominantly in oilseed rape. The pathogen enters hosts through the root and subsequently entertains a parasitic life stage in the xylem before invading other tissues late in the infection cycle. Using Arabidopsis thaliana wild-type and mutants in major defence pathways, we have analysed the root transcriptomes at 10 days after inoculation (dpi). At this time point, nearly all of the 661 induced genes were expressed independently of the defence hormones jasmonic acid (JA) and salicylic acid (SA). Intriguingly, over $25 \%$ of these genes were constitutively expressed in mock-treated coronatine insensitivie1 (coi1) plants, which are deficient in JA perception. Since constitutive expression levels in coi1 were in a similar range as in $V$. longisporum-infected plants, we postulate that induction of these genes is mediated by the
\end{abstract}


systemic inactivation of COI1. V. longisporum-induced/COI1-repressed genes were related to SA-dependent defence responses and included the master regulator of SA signalling, SYSTEMIC ACQUIRED RESISTANCE DEFICIENT 1 (SARD1). Mutating SARD1 and its homolog CALMODULIN-BINDING PROTEIN 60-LIKE G interfered with Verticillium-induced expression and with constitutive expression of these genes in the coi1 background. In contrast, overexpression of $S A R D 1$ did not lead to enhanced expression of target genes, most likely because the negative effect of COl1 was not overcome.

\section{Introduction}

Plant roots are in close contact with a plethora of commensal, mutualistic and pathogenic microorganisms densely populating soil environments. Interactions with commensals and mutualists are beneficial for plant health, whereas pathogenic microorganisms can cause severe damage to plants (Raaijmakers et al., 2009; Berendsen et al., 2012; Mauchline and Malone, 2017).

All microorganisms carry some form of microbe-associated molecular pattern (MAMP). MAMPs are essential conserved molecules like flagellin, chitin or NLPs (Necrosis and ethylene-inducing peptide 1 (Nep1)-like proteins) that are perceived by pattern recognition receptors (PRRs) on the plant's plasma membrane (Bittel and Robatzek, 2007; Boller and Felix, 2009; Newman et al., 2013; Oome et al., 2014). Upon detection of these MAMPs, plant defences are activated including the synthesis of plant hormones. The corresponding hormone-mediated signalling pathways lead to massive transcriptional reprogramming to generate appropriate defence outputs against pathogen attack. The two main defence pathways are the salicylic acid (SA)-mediated defence and the jasmonic acid (JA)/ethylene (ET)-mediated defence.

Crucial in activating the SA pathway are the two transcription factors SYSTEMIC ACQUIRED RESISTANCE DEFICIENT 1 (SARD1) and CALMODULIN-BINDING PROTEIN 60-LIKE G (CBP60g), which start SA synthesis by inducing expression of the enzymes ISOCHORISMATE 
SYNTHASE 1 (ICS1) and avrPphB SUSCEPTIBLE 3 (PBS3) (Strawn et al., 2007; Wang et al., 2009; Zhang et al., 2010; Sun et al., 2015). Downstream of SA biosynthesis, an indispensable component of the SA signalling cascade is NONEXPRESSER OF PATHOGENESISRELATED GENES 1 (NPR1), which interacts with transcription factors of the TGACG-motif binding (TGA) family to coordinate massive transcriptional reprogramming (Cao et al., 1994; Cao et al., 1997; Zhang et al., 2003; Rochon et al., 2007). Transcriptional reprogramming in the JA defence pathway is initiated by degradation of repressors of the JASMONATE ZIMdomain (JAZ) family, which block transcription factors like MYC2, 3 and 4, or ETHYLENEINSENSITIVE3 (EIN3) on promoters of JA- and JA/ET-responsive genes (Chini et al., 2007; Thines et al., 2007; Fernández-Calvo et al., 2011). The bioactive JA conjugate jasmonoylisoleucine (JA-lle) facilitates binding of the F-box protein CORONATINE INSENSITIVE 1 (COI1) as part of the $\mathrm{SCF}^{\mathrm{COI1}}$ complex to the JAZ repressors (Sheard et al., 2010). The SCF ${ }^{\mathrm{CO} 11}$ complex constitutes a functional E3 ligase (Xu et al., 2002). Upon co-reception of JA-lle by COI1 and JAZs, JAZs are polyubiquitinated and degraded via the 26S proteasome.

Initiation of the SA or JA/ET pathway is based on the type of pathogen invading. Pathogens can be divided into different classes depending on their lifestyle; biotrophic pathogens feed off living hosts, whereas necrotrophic pathogens kill hosts to feed on the dead plant matter (Glazebrook, 2005). Hemibiotrophs employ a transitional lifestyle; they start off as biotrophs, often spreading widely in the host plant during this initial phase, and then turn necrotrophic feeding off dead host tissue (Horbach et al., 2011). Generalised, SA-mediated defence responses are deployed against biotrophic pathogens and the JA/ET defence pathway is launched against necrotrophic pathogens (Glazebrook, 2005).

The ascomycete fungus Verticillium longisporum is a hemibiotrophic soil-borne pathogen with a host range largely restricted to Brassicaceae (Depotter et al., 2016). Mainly Brassica napus is an economically important host crop in Europe, to whose production $V$. longisporum poses an increasing threat (Depotter et al., 2016). V. longisporum penetrates roots and uses xylem vessels to spread systemically in its host. Infection with $V$. Iongisporum causes stunted growth, 
vein clearing, leaf chlorosis and premature senescence (Reusche et al., 2012; Depotter et al., 2016).

V. longisporum, as well as another soil-born vascular ascomycete fungus, Fusarium oxysporum, have been shown to require the JA-Ile receptor COI1 for successful infection of Arabidopsis plants (Thatcher et al., 2009; Ralhan et al., 2012). Infected coi1 mutants show less severe disease symptoms in shoots where lower fungal amounts were detected at late stages of infection. In contrast, the JA biosynthesis mutant allene oxide synthase (aos) shows wild-type (WT)-like symptoms (Ralhan et al., 2012). Reciprocal grafts between scions and roots of coi1 and WT revealed that COI1 is required in roots to cause susceptibility to $F$. oxyposum and V. longisporum (Thatcher et al., 2009; Ralhan et al., 2012).

Prompted by these findings, we have recently shown that in Arabidopsis roots, COI1 acts as repressor of defence gene expression (Ulrich et al., 2021). RNA-seq analysis revealed that in coi1 roots a number of SA defence-related genes are basally de-repressed. Again, aos mutant roots behave like WT roots and show no such de-repression. This repressor function of COI1 is uncoupled from its role in the JA pathway as it does not require JA-Ile and most likely no interaction with JAZ proteins (Ulrich et al., 2021). It remains to be elucidated how this COl1mediated repression is mechanistically achieved. In the setup used for our previous RNA-seq analysis, however, we could not gain any information on how coi1 and susceptible plant roots react to infection with $V$. longisporum as axenically grown plantlets did not show significant responses to infection on the transcriptional level.

Here we present data from a new RNA-seq analysis of $V$. longisporum-infected coi1, aos, WT and SA biosynthesis-impaired sid2 (salicylic acid induction-deficient 2) roots. This time, plants were grown in soil during the infection and a number of genes were induced in all genotypes. Intriguingly, roughly $25 \%$ of these genes were pre-induced in coi1. We furthermore show that transcription factor SARD1 is the master regulator of this group of genes, facilitating their increased expression in both coi1 roots and after infection. However, overexpression of SARD1 in WT roots could not induce gene expression on its own. Hence, we conclude that, besides SARD1 binding, induction of these genes in response to $V$. longisporum infection 
additionally requires a second yet unknown mechanism, potentially the inactivation of COI1 as a repressor. This inactivation of $\mathrm{COI} 1$ does not seem to be required in shoot tissue, correlating with the fact that in shoots COl1 does not act a repressor on the same set of genes. It is tempting to speculate that $\mathrm{COI} 1$ might have a role in attenuating SA responses in roots until a strong defence response becomes indispensable.

\section{Results}

\section{A subgroup of COl1-repressed genes is induced in roots after infection with $V$. longisporum}

As the coi1 mutant is tolerant against infection with $V$. longisporum, we were interested to see how the root transcriptome of coi1 plants differs from that of susceptible plants. Since the phenotypes of the JA biosynthesis mutant aos and the JA receptor mutant coi1-t differ after infection, we also included aos plants in the analysis. We also analysed the SA biosynthesis mutant sid2-2 in order to identify potential effects resulting from interactions between the defence pathways. As coi1-t and aos plants are male sterile, we genotyped the plantlets resulting from heterozygous seed batches before experiments and also included the respective outcrossed WT plants from each population in the RNA-seq analysis. RNA was derived from four experiments, each comprising combined roots from twelve plants per genotype and treatment. Tissue was harvested at $10 \mathrm{dpi}$ or 10 days after mock treatment. We chose this time point as we expected that at $10 \mathrm{dpi}$ the root was sufficiently colonised by the fungus to observe robust responses. At this time point no differences in fungal load in the shoot can be detected between susceptible WT and aos plants and tolerant coi1 plants (Ralhan et al., 2012). Thus, differences in infection-specific transcript levels in the different genotypes are unlikely to be due to different fungal loads.

We obtained a first impression of the root transcriptomes by principal component analysis (Figure 1a). Mock-treated samples of aos, $\mathrm{WT}_{\text {aos }}, \mathrm{WT}_{\text {coit-t }}$ and sid2-2 are closely grouped with clear separation from their infected counterparts, which also group together. Mock-treated 
coi1-t roots are clearly different from the other four mock-treated genotypes. This difference between coi1-t and the other genotypes is less pronounced at $10 \mathrm{dpi}$.

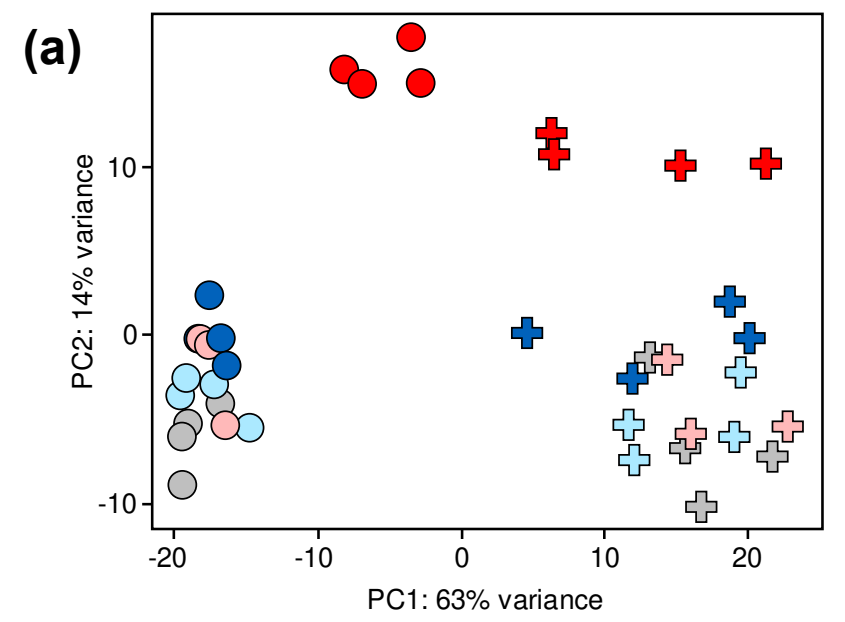

$$
\begin{aligned}
& \mathrm{WT}_{\text {aos }} \text { mock } \\
& \text { 乌 } \mathrm{WT}_{\text {aos }} 10 \mathrm{dpi} \\
& \mathrm{WT}_{\text {coit-t }} \text { mock } \\
& \text { 乌 WT } T_{\text {coit-t }} 10 \mathrm{dp} \\
& \text { aos mock } \\
& \text { aos } 10 \mathrm{dpi}
\end{aligned}
$$

\section{(b)}

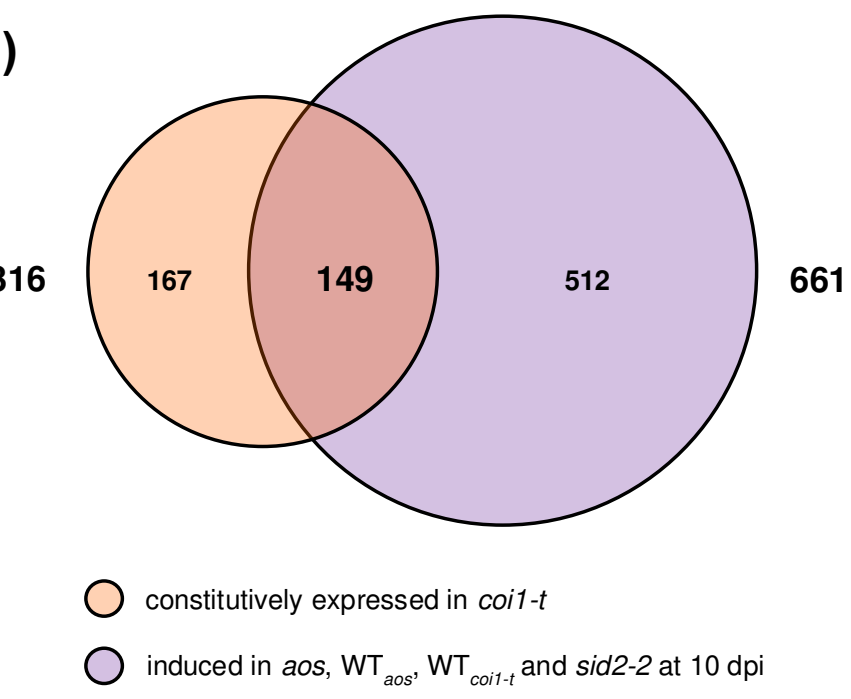

Figure 1. A group of 149 genes is de-repressed in coi1-t roots and induced in aos, WT $\mathrm{WT}_{\text {coi1-t }}$, and sid2-2 at $10 \mathrm{dpi}$.

(a) Principal component analysis of the normalised root transcriptome data acquired by RNA-seq analysis 10 days after mock treatment or inoculation with $1 \times 10^{6}$ spores $/ \mathrm{mL}$ sGFP-expressing $V$. longisporum. Biological replicates from four independent experiments are symbolised by circles (mock) or plus signs (10 dpi). For $W_{\text {coir-t } t}$ only three replicates were analysed for both mock and $10 \mathrm{dpi}$ treatments. WTaos and $W T_{\text {coilt-t }}$ are the wild-types obtained from the segregating offspring of heterozygous aos and coi1-t seeds. (b) Venn diagram showing the overlap between 316 genes constitutively upregulated in mock-treated coi1-t roots vs mock-treated aos, $\mathrm{WT}_{\text {aos, }}, \mathrm{WT}_{\text {coil-t }}$ and sid2-2 (> 2-fold, $\mathrm{p}<0.05$ ) and 661 genes induced in aos, WTaos, WT coir-t and sid2-2 at 10 dpi (> 2-fold, $\mathrm{p}<$ 0.05). Expression data was obtained by RNA-seq analysis from root material 10 days after mock treatment or inoculation with $1 \times 10^{6}$ spores $/ \mathrm{mL}$ sGFP-expressing $\mathrm{V}$. longisporum. Circles are drawn to scale with respect to the number of genes represented in each group. 
After infection, gene induction is similar in both WTs $(>2$-fold, $p<0.05)$ (Figure S1a, Table 1 and subtables). When intersecting gene induction responses in both WTs there is $12.4 \%$ and $18.6 \%$ drop out on either side, mostly of genes that closely miss the threshold (Figure S1a). Generally, the responses induced in WT plants are related to cell wall biogenesis, xylem development and SA defence responses (Figure S2). Gene induction after infection in WT aos shows $84.8 \%$ overlap with gene induction in the aos mutant (Figure S1b). Moreover, both WTs show largely overlapping gene induction patterns with the sid2-2 mutant after infection (Figure S1c and S1d). This indicates that there are no major differences between the WTs and either of the JA or SA biosynthesis mutants, as suggested by the PCA analysis (Figure 1a). Hence, we continued our analysis with the genes most robustly differentially regulated, i.e. those that are induced in all four genotypes $\mathrm{WT}_{\text {aos, }} \mathrm{WT}_{\text {coir-t, }}$ aos and sid2-2. After infection a total of 661 genes are upregulated in these genotypes (Table 1 and subtables). Together in aos, sid2-2 and the WTs, 91 genes are downregulated after infection ( $>2$-fold, $p<0.05$ ) (Table 1 and subtables).

At $10 \mathrm{dpi}$, eleven genes were lower expressed in coi1-t roots compared to all other genotypes (> 2-fold, $p<0.05$ ) (Table 1 and subtables). Of these, only three genes are induced after infection in aos, both WTs and sid2-2. At $10 \mathrm{dpi}, 71$ genes are higher expressed in coi1-t compared to the other four genotypes, of which seven genes are inducible in $\mathrm{WT}_{\text {aos, }}, \mathrm{WT}_{\text {coir-t, }}$ aos and sid2-2 (Table 1 and subtables).

The greatest difference observed between coi1-t roots and the other genotypes is a set of 316 genes higher expressed in mock-treated coi1-t roots (>2-fold, $p<0.05$ ) (Table 1 and subtables). As these genes are not de-repressed in aos, this expression pattern seems to be specific for coi1 roots and not due to disruptions in the JA signalling pathway. Only eight genes are lower expressed in mock-treated coi1-t roots compared to aos, both outcrossed WTs and sid2-2 ( $>2$-fold, $p<0.05)$.

The 661 genes induced after infection in aos, WT aos, WT coit-t and sid2-2 share an overlapping 149 genes with the group of 316 genes de-repressed in coi1-t roots (Figure $1 \mathrm{~b}$ and Table 1 
and subtables). Of these 149 genes, only 21 genes are higher expressed in coi1-t at $10 \mathrm{dpi}$ compared to mock treated coi1-t roots (Table 1 and subtables).

The genes found to be de-repressed here are largely overlapping with the set of genes we have previously reported to be suppressed by COI1 in roots (Ulrich et al., 2021). This interesting group of 149 genes identified here raises the question whether activation of gene expression after infection with $V$. longisporum is achieved via the inactivation of the COl1mediated repression.

The subgroup of COl1-suppressed and $V$. longisporum-responsive genes is related to SA-mediated defence responses and is significantly enriched with the 'GAAATTT' motif GO term analysis showed that the 149 genes de-repressed in coi1-t and induced in aos, both WTs and in sid2-2 roots after infection are associated with immune defence responses, in particular SA-mediated defence responses (Figure 2). Similarly, the 167 genes under negative control of COI1 and not induced after infection, are also associated with SA defence responses (Figure S3a). In contrast, the 512 genes induced after infection in aos, both WTs and sid2-2 but not under control of COI1 are not associated with SA-mediated defences but with various processes in cell wall production (Figure S3b).

Using motif enrichment analysis, we aimed to determine if a certain transcription factor binding motif was overrepresented in the regulatory regions of the different clusters of genes we had identified in the RNA-seq, specifically in the 149 genes de-repressed in coil and induced after infection. The Motif Mapper cis-element analysis tool scans 1-kb sequences upstream of predicted transcriptional start sites (Berendzen et al., 2012). The average number of detected binding motifs in a specific gene set is compared to the average number of found binding motifs randomly obtained 1000 times from a chosen control set. We screened for enriched motifs in the five clusters of genes defined from the RNA-seq data (shown in Figure 1b): The two main groups of 316 genes de-repressed in coi1 roots and 661 genes induced after infection in susceptible genotypes, along with the subgroups identified; the 149 genes de-repressed in 


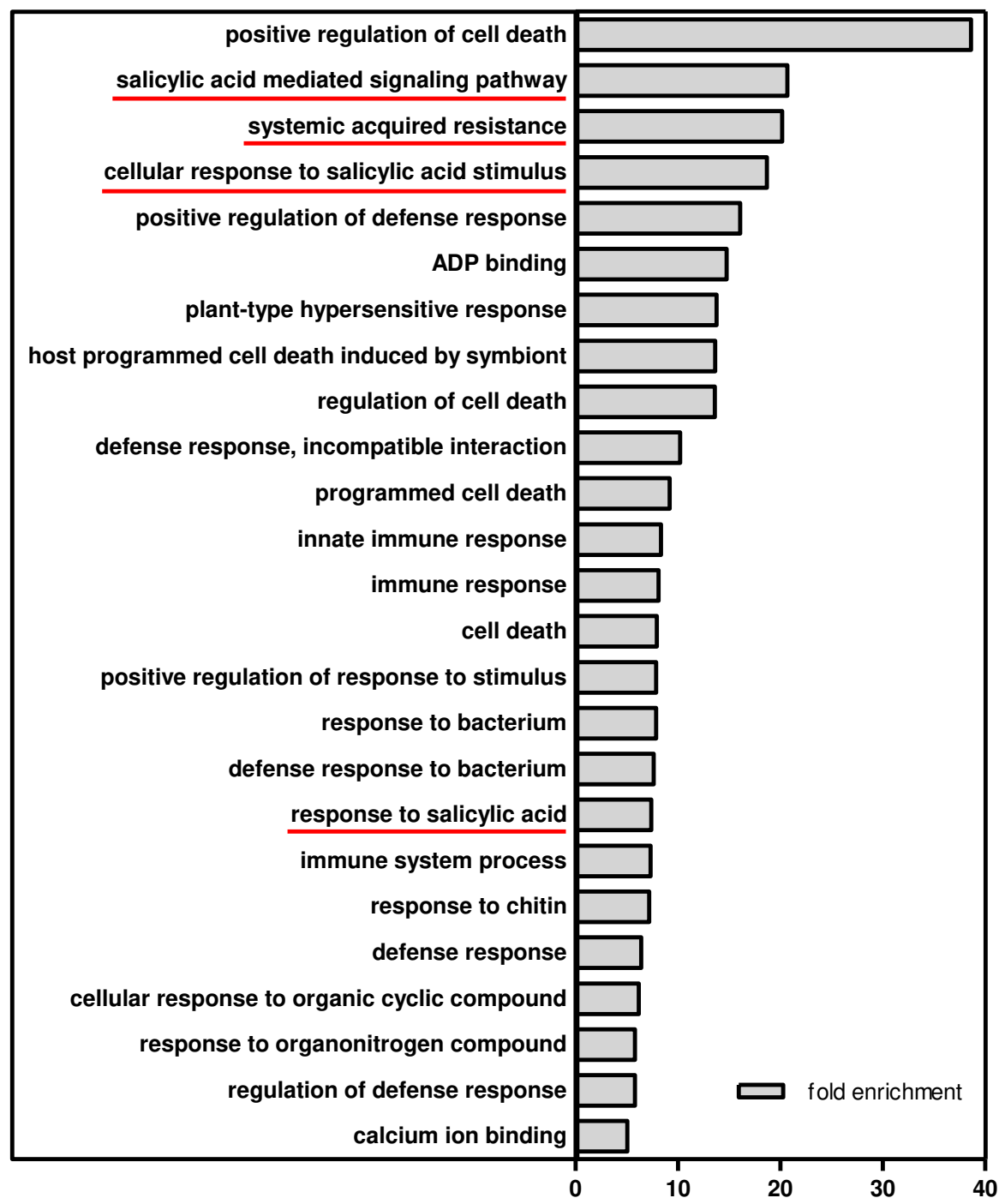

Figure 2. The 149 genes de-repressed in coi1 and induced after infection are related to defence responses.

Gene Ontology (GO) term enrichment analysis of the 149 genes basally upregulated in coi1- $t$ compared to aos, $\mathrm{WT}_{\text {aos }}, \mathrm{WT}_{\text {coit-t }}$ and sid2-2 (> 2-fold; $p<0.05$ ) and induced in aos, $\mathrm{WT}_{\text {aos }}, \mathrm{WT}_{\text {coit-t } t}$ and sid2-2 at 10 dpi (> 2-fold, $p<0.05$ ). Bars represent the fold enrichment of the number of genes found per GO term in the group of 149 genes against the number of genes found within the Arabidopsis genome associated with that GO term. Only GO terms with > 5-fold enrichment against the genome are shown. $S A$ defence related GO terms are underlined in red.

coi1 and induced after infection, the 167 genes de-repressed in coi1 but not induced after infection and the 512 genes induced after infection but not under control of COI1.

Analysis of the regulatory regions showed that the 'GAAATTT' motif was significantly enriched in the promoters of the 149 genes de-repressed in coi1 and induced after infection compared to promoter sequences randomly drawn from the entire genome (Figure 3a). 'GAAATTT' is the 
binding motif for SARD1 and CBP60g. This binding motif was not enriched in any of the other groups of genes.

(a)

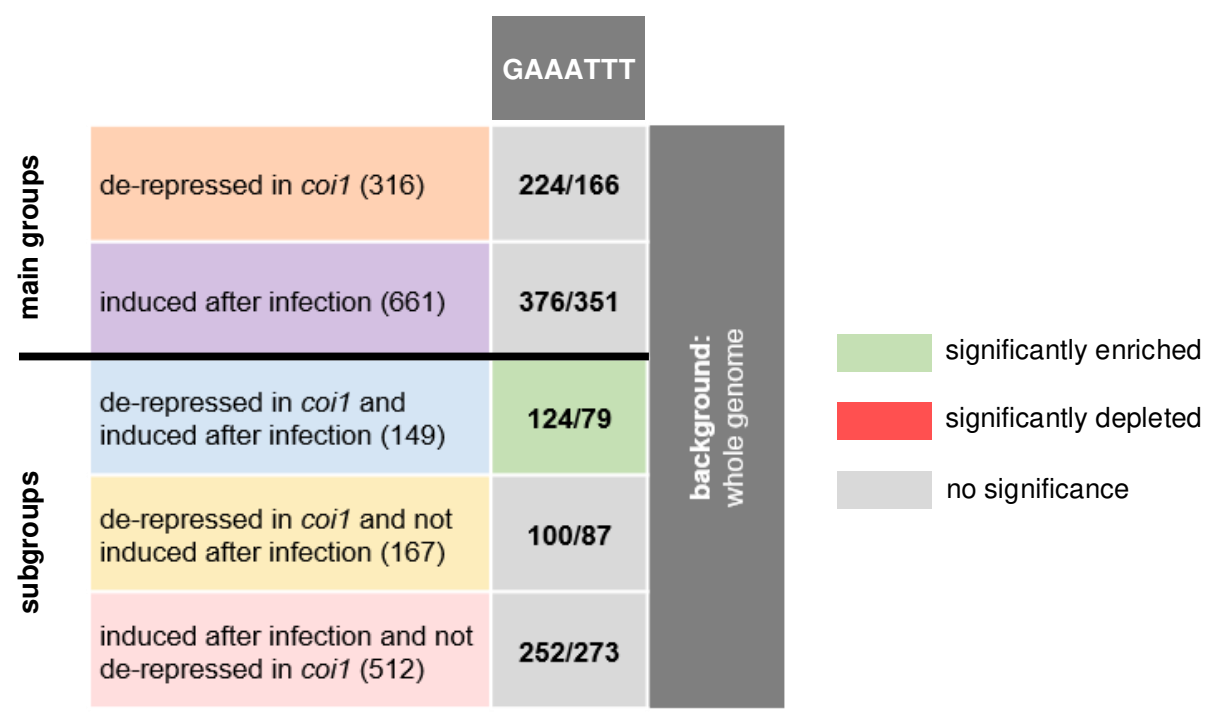

(b)

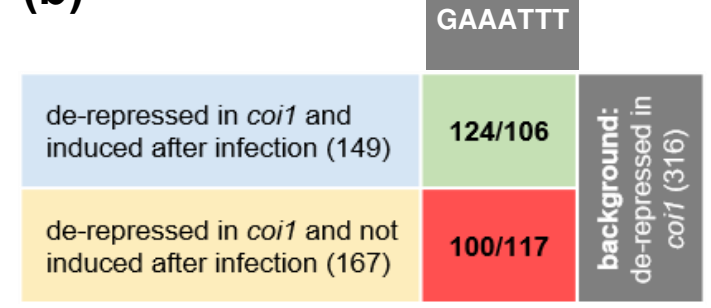

(c)

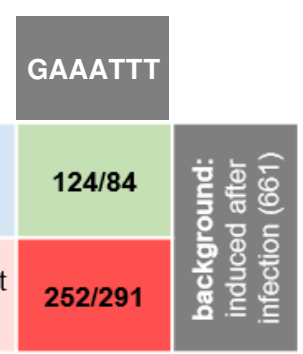

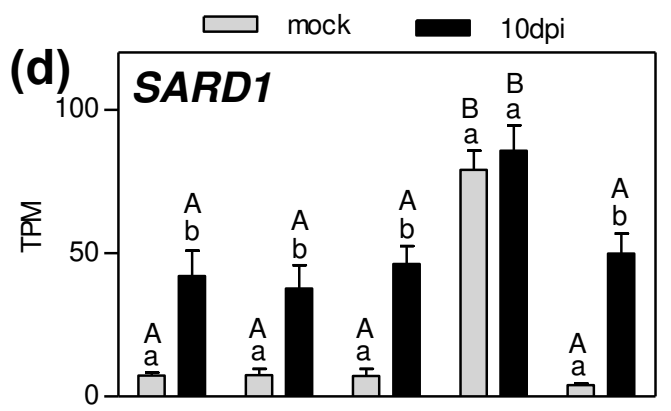
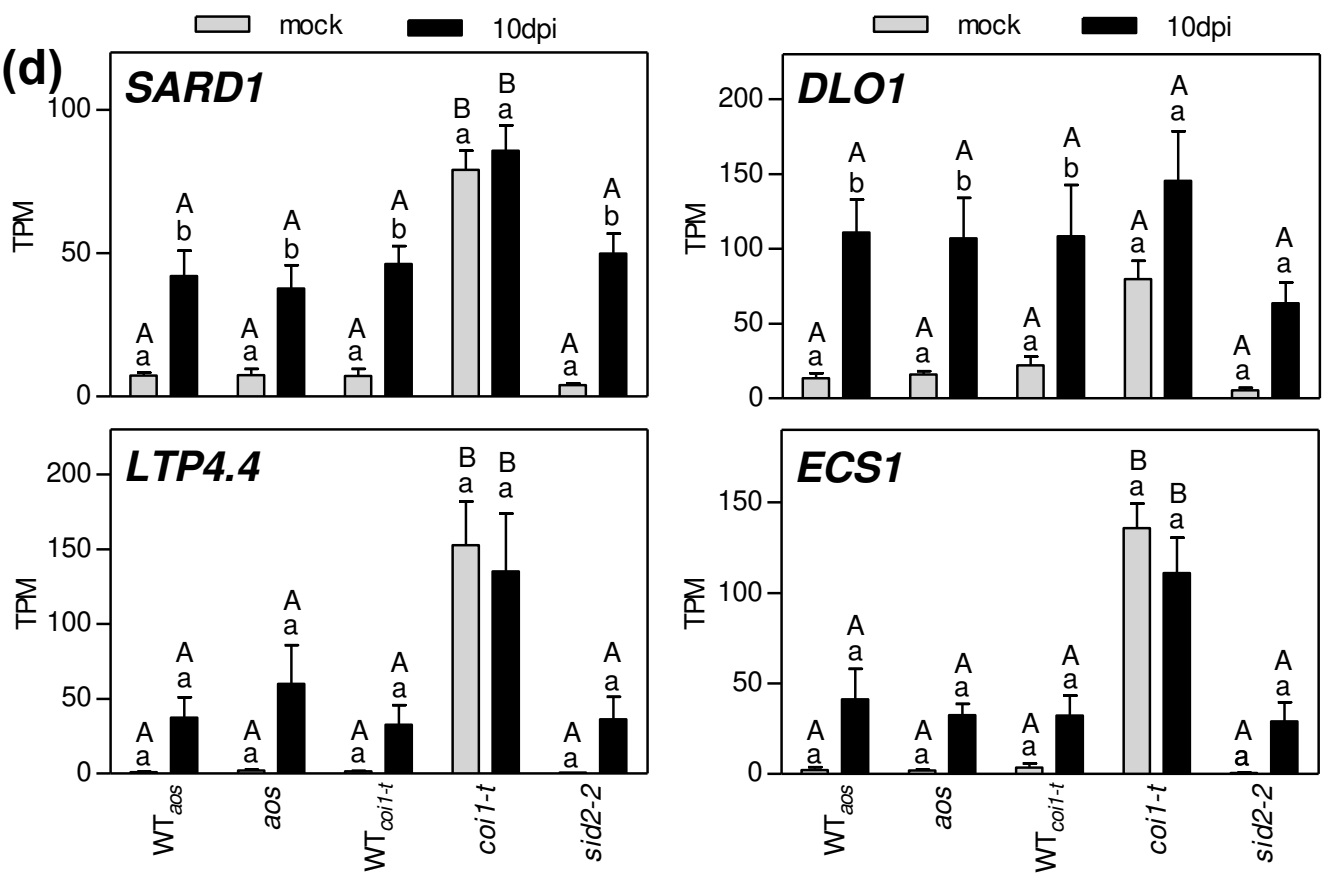

Figure 3. The binding motif for SARD1 and CBP60g 'GAAATTT' is significantly enriched in the 149 defence genes of interest.

Full legend on next page. 
Figure 3. The binding motif for SARD1 and CBP60g 'GAAATTT' is significantly enriched in the 149 defence genes of interest.

Motif Mapper cis-element analysis of (a) the 316 genes de-repressed in coi1-t roots (coi1-t mock > aos, WTaos, WT coir-t, sid2-2 mock; 2-fold; $\mathrm{p}<0.05$ ), the 661 genes induced after infection (aos, WTaos,

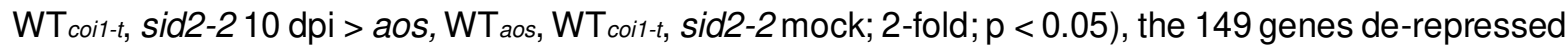
in coi1- $t$ and induced after infection, the 167 genes de-repressed in coi1-t and not induced after infection and the 512 genes induced after infection but not de-repressed in coi1-t (b) the 149 genes de-repressed in coi1-t and induced after infection and the 167 genes de-repressed in coi1-t and not induced after infection against the background of the set of all 316 genes de-repressed in coi1-t roots (c) the 149 genes de-repressed in coi1-t and induced after infection and the 512 genes induced after infection and not de-repressed in coi1-t roots against the background of the set of all 661 genes induced after infection. Numbers before slashes show the total number of detected motifs in the set of interest. Numbers behind the slashes show the number of expected motif counts in a set of randomly chosen promoters within the indicated background set. Significant enrichment/depletion is defined at $p<0.05$. (d) Relative expression of SARD1, DLO1, LTP4.4 and ECS1 transcript levels as quantified by RNA-seq analysis 10 days after mock treatment or inoculation with $1 \times 10^{6}$ spores $/ \mathrm{mL}$ sGFPexpressing $V$. longisporum. Bars are means of Transcripts Per Million (TPM) \pm SEM of three to four biological replicates of each genotype, with each replicate representing twelve roots from one independent experiment. For statistical analysis, a two-way ANOVA was performed followed by Bonferroni's multiple comparison test; lowercase letters denote significant differences within each genotype between mock and $10 \mathrm{dpi}(p<0.05)$, uppercase letters denote significant differences between genotypes subjected to the same treatment $(p<0.05)$. WTaos and WT coir-t are the two wildtypes lines obtained from the segregating offspring of heterozygous aos and coi1-tseeds.

When testing the groups of the 149 inducible and 167 non-inducible genes under COI1-control against the total 316 genes de-repressed in coi1 rather than the whole genome, the 149 inducible genes were enriched with the 'GAAATTT' motif (Figure 3b). The other 167 genes, however, were actually depleted of the motif.

Similarly, we analysed all 661 genes inducible after infection split into two groups of those 149 under negative control of $\mathrm{COI} 1$ and those 512 not suppressed by COl1. Only the group of the 149 genes showed enrichment of the 'GAAATTT' motif. The 512 genes not under COI 1 control were significantly depleted of the motif (Figure 3c).

Overall, this analysis shows that out of all groups analysed, the 149 genes induced after infection and de-repressed in coi1 are explicitly enriched with the SARD1/CBP60g binding motif. 
Indeed, SARD1 might be a promising candidate for regulating this set of genes as it is found within the group of the 149 genes. Figure 3d shows expression patterns of SARD1 and three representative marker genes chosen from the 149 genes in this group: LIPID TRANSFER PROTEIN 4.4 (LTP4.4) (AT5G55450), a gene involved in SA catabolism DMR6-LIKE OXYGENASE 1 (DLO1) (AT4G10500) and the cell wall protein ECS1 (formerly CXC750) (AT1G31580).

\section{COl1 does not suppress target gene expression in shoots}

Seeing that COI1 represses basal expression of 316 genes in roots, we analysed gene expression in whole shoots from the same plants whose roots underwent the RNA-seq analysis (Figure 4). In contrast to roots, our marker genes were not de-repressed in coi1 shoots. Hence, the observed suppressive action of COI1 on gene expression is exclusive to roots.
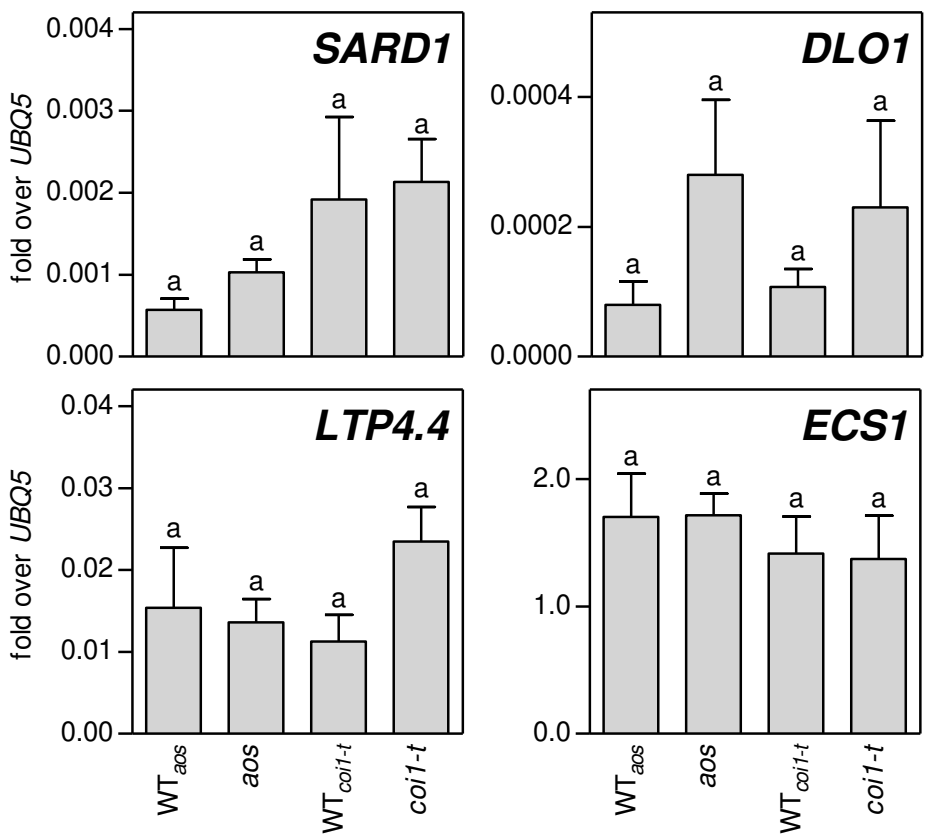

Figure 4. Unlike in roots, COI1 is not a constitutive repressor of target genes in shoots.

SARD1, LTP4.4, DLO1 and ECS1 transcript levels, measured by qRT-PCR. RNA was extracted from shoots 10 days after mock treatment from the same plants whose roots were subjected to the RNA-seq. Bars are means \pm SEM of four replicates, each made up twelve shoots per genotype. For statistical analysis, a one-way ANOVA was performed followed by Tukey's multiple comparison test; lowercase letters denote significant differences between samples $(p<0.05)$. WT $\mathrm{Wos}_{\text {aos }}$ and $\mathrm{WT}_{\text {coil-t }}$ are the two wildtypes lines obtained from the segregating offspring of heterozygous aos and coi1-t seeds. 


\section{Gene induction after $V$. longisporum infection does not require increased SA levels}

GO term analysis linked the genes under negative control of COI1 and induced after infection to defence responses, especially SA-mediated defences. In contrast to this, we see our genes of interest induced after infection in sid2 roots in the RNA-seq data. We repeated experiments not only in sid2 roots but also included npr1 mutants to see if the observed gene induction requires the main hub of SA-mediated transcriptional reprogramming. Again, induction of SARD1, LTP4.4 and DLO1 was similar in WT and sid2 roots, confirming observations from the RNA-seq (Figure 5a). ECS1 showed slightly lower expression in infected sid2 mutants compared to WT but was still almost 6-fold induced over mock plants. Induction of all genes was still observed in infected npr1 mutants, albeit to slightly smaller extent in some cases. We measured SA levels in WT and sid2 mutants and could not detect an increase in SA levels in either of the two genotypes after infection (Figure S4).

This seemingly contradictory fact that SA-defence related genes are upregulated without increased SA levels is not exclusive to $V$. longisporum-infected roots. Local infiltration of leaves with nlp14, a 14 amino acid-long immunogenic peptide found in NLPs, also induced expression of SARD1 and LTP4.4 after 24 hours in sid2 and npr1 mutant leaves (Figure 5b). DLO1 and ECS1 expression, however, was ICS1- and NPR1-dependent under these conditions.

In coi1 roots, ICS1 is de-repressed and SA levels elevated (Figures S5a and S5b). Still, basal upregulation of SARD1, LTP4.4 and ECS1 was still elevated in coi1 sid2 double mutant roots, which have WT-like SA levels (Figures S5b and S5c). Elevated DLO1 expression was dependent on upregulated SA levels though.

Our genes of interest can be regulated independently from increased SA levels after $V$. longisporum infection, and mostly so in locally nlp14-triggered leaves and coi1 roots. Nevertheless, we could establish a connection to SA signalling. Treatment of roots with SA showed that our marker genes - with the exception of LTP4.4 - are responsive to SA accumulation (Figure S6). Induction after 24 hours of SA treatment requires NPR1 and hence 
(a)
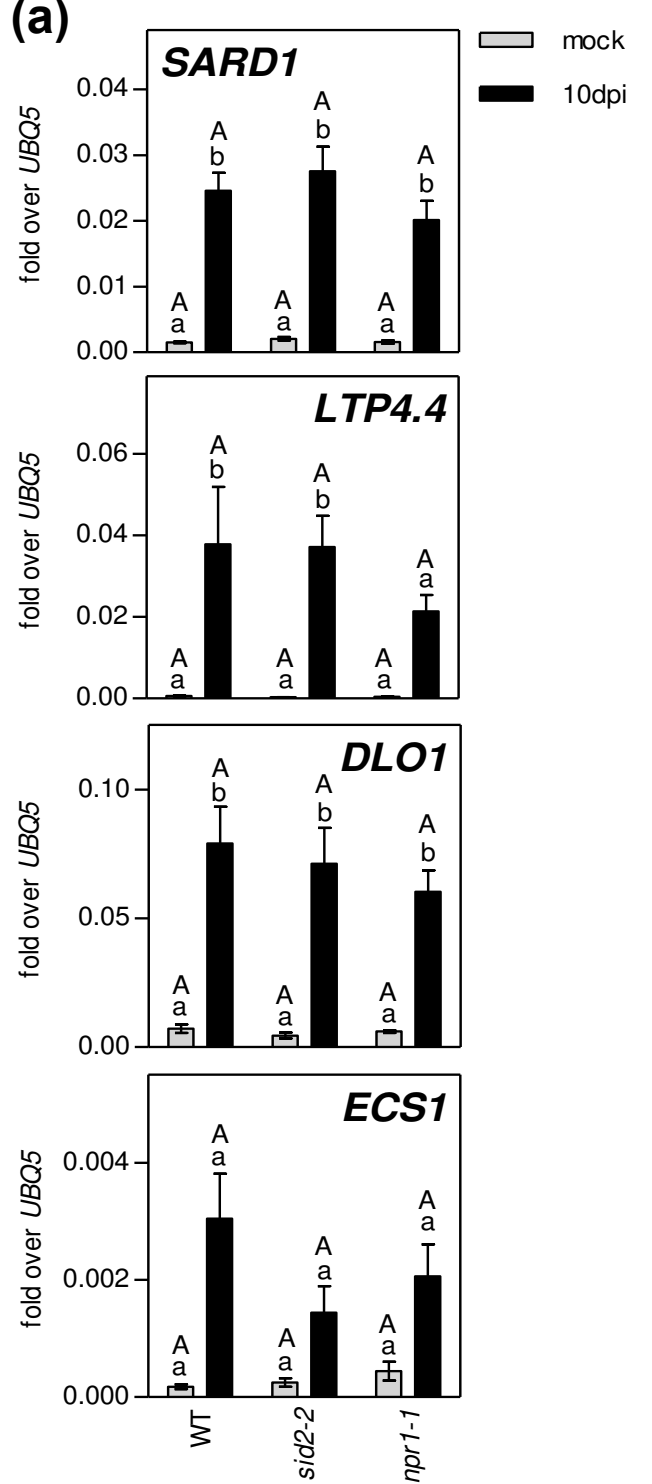

(b)
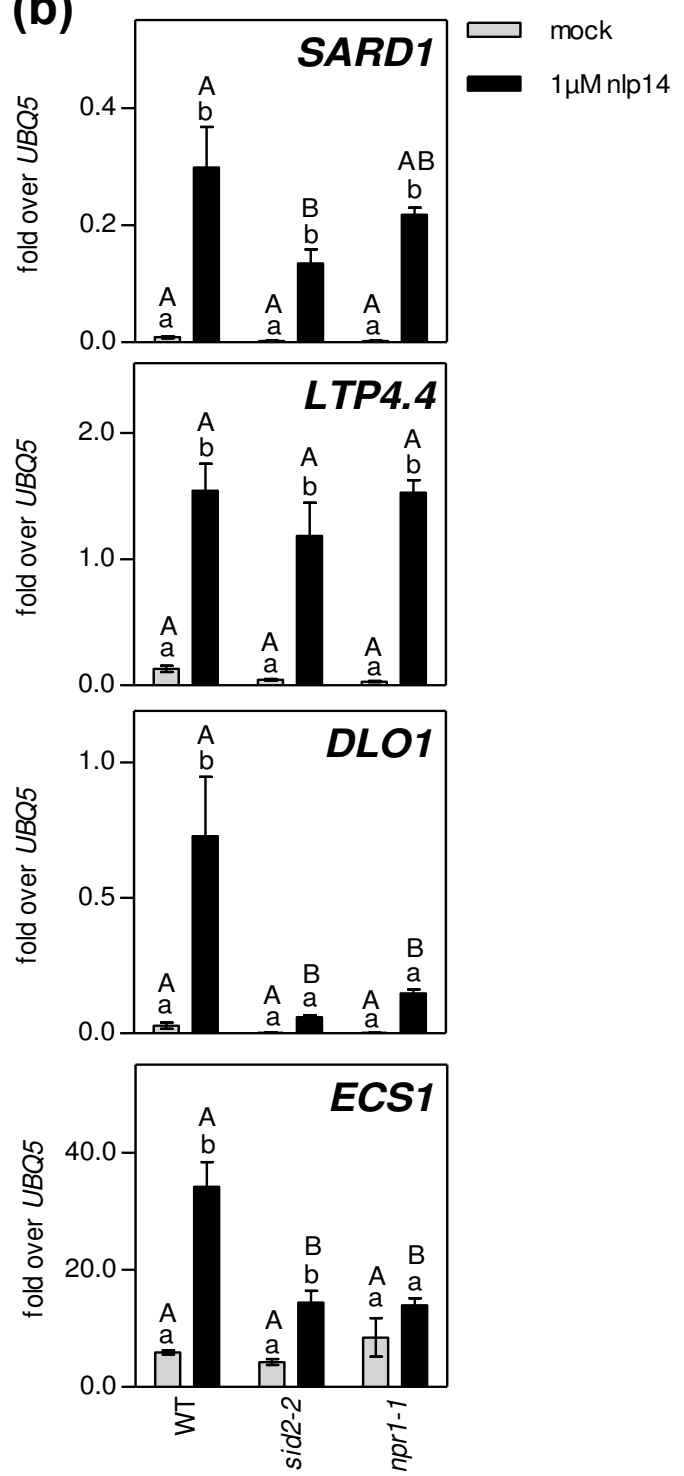

Figure 5. Genes of interest are induced independently of increased SA levels and NPR1 in roots.

(a) SARD1, LTP4.4, DLO1 and ECS1 transcript levels, measured by qRT-PCR. RNA was extracted from roots 10 days after mock treatment or infection with $1 \times 10^{6}$ spores $/ \mathrm{mL} \mathrm{V}$. longisporum. Bars are means \pm SEM of thirteen to sixteen roots per genotype. For ECS1 transcript levels in npr1-1 only six replicates are shown. For statistical analysis, a two-way ANOVA was performed followed by Bonferroni's multiple comparison test; lowercase letters denote significant differences within each genotype between mock and $10 \mathrm{dpi}(p<0.05)$, uppercase letters denote significant differences between genotypes subjected to the same treatment $(p<0.05)$. (b) SARD1, LTP4.4, DLO1 and ECS1 transcript levels, measured by qRT-PCR. RNA was extracted from leaves 24 hours after infiltration with $1 \mu \mathrm{M}$ nlp14. Bars are means $\pm \mathrm{SEM}$ of four to six replicates per genotype. For statistical analysis, a two-way ANOVA was performed followed by Bonferroni's multiple comparison test; lowercase letters denote significant differences within each genotype between mock and $1 \mu \mathrm{M}$ nlp14 $(p<0.05)$, uppercase letters denote significant differences between genotypes subjected to the same treatment $(p<0.05)$. 
classical SA defence signalling. Exogenous treatment with SA even hyperinduced gene expression levels compared to the expression level in coi1 roots for DLO1 and ECS1.

In summary, our genes of interest are connected to classical SA signalling but under the conditions we investigate here, they are regulated independently of increased SA levels.

\section{SARD1/CBP60g are required for gene induction after $V$. longisporum infection and for basal gene upregulation in coi1 roots}

We next turned our attention to the role of SARD1 in regulating our genes of interest, as motif mapping analysis had shown significant enrichment of the SARD1/CBP60g binding motif in their promoters (Figure 3a, 3b and 3c). SARD1 and CBP60g are close homologues that bind to the same motif. Both transcription factors are important for local and systemic pathogen defence responses by regulating key players in SA and N-hydroxy-pipecolic acid synthesis like ICS1, avrPphB SUSCEPTIBLE3 (PBS3), FLAVIN-DEPENDENT MONOOXYGENASE1 (FMO1) and AGD2-LIKE DEFENSE RESPONSE PROTEIN1 (ALD1) (Wang et al., 2009, 2011; Zhang et al., 2010a; Sun et al., 2015). Moreover, they bind to promoters of a plethora of other defence response genes, such as key players in ETI and PTI, e.g. ENHANCED DISEASE SUSCEPTIBILITY1 (EDS1), PHYTOALEXIN DEFICIENT4 (PAD4), BRASSINOSTEROID INSENSITIVE 1 (BAK1) and BOTRYTIS-INDUCED KINASE1 (BIK1) (Sun et al., 2015). Even though CBP60g is not induced after infection (Figure S7), we decided to work with sard1 cbp60g double mutants to exclude any possible compensatory effects in gene activation by CBP60g in the absence of SARD1.

To determine if SARD1/CBP60g are required for gene induction after infection with $V$. longisporum, we infected sard1 cbp60g mutants and determined marker gene expression at $10 \mathrm{dpi}$. As a well-established target gene of SARD1 and CBP60g we also included ICS1 in our analysis (Zhang et al., 2010b; Sun et al., 2015). As shown above (Figure S5a), ICS1 is under negative control of COI1 and even though it was not significantly induced after infection in the RNA-seq data, we always observed minor ICS1 induction in all subsequent infections 
experiments. The observed induction of ICS1 was usually lower than 2-fold, therefore, it is not as strongly responsive to $V$. longisporum infection as our other marker genes (Figure 6a). Overall, in sard1 cbp60g roots all four marker genes, LTP4.4, DLO1, ECS1 and ICS1 showed greatly impaired gene induction compared to WT roots at 10 dpi (Figure 6a).

Having established that SARD1/CBP60g are crucial for induction of marker genes in response to infection, we were interested if they are also involved in enhanced gene expression in coi1 roots. To determine if this was the case, we crossed the sard1 cbp60g mutant into the coi1 background. In the coi1 sard1 cbp60g triple mutant expression of our marker genes was reverted back to WT levels (Figure 6b). Hence, SARD1/CBP60g are also responsible for the upregulated expression of LTP4.4, DLO1, ECS1 and ICS1 in coi1 roots.

Motif mapping had shown that the 'GAAATTT' motif was depleted in the groups of the 167 genes de-repressed in coi1 and not significantly induced after infection as well as in the 512 genes induced after infection but not under control of COI1. We randomly selected a few genes from each of these two groups and assessed if they are regulated independently of SARD1/CBP60g.

Testing expression of genes found in the 167 COl1-repressed but non-inducible genes, we saw that a RmIC-like cupins superfamily protein (AT5G39120) was still de-repressed in coi1 sard 1 cbp60g roots. WRKY54 and WRKY46 expression was fully and largely SARD1/CBP60gdependent, respectively (Figure S8a). However, upregulation of WRKY54 in coi1 is ICS1dependent (Figure S8b), hence the downregulation in coi1 sard1 cbp60g is likely due to the reduction of ICS1-derived SA levels (Figure 6b). WRKY46 is still upregulated in coi1 sid2 mutants confirming that it is mostly SARD1/CBP60g-dependent (Figures S8a and S8b). Two genes picked from the group of the 561 genes induced after infection but not suppressed by COI1, ANAC076 and ERF54, were still inducible in sard1 cbp60g mutants after infection (Figure S8c).

Taken together, these observations show that SARD1/CBP60g are necessary for activation of our defence genes of interest in $V$. longisporum-infected and coi 1 roots 

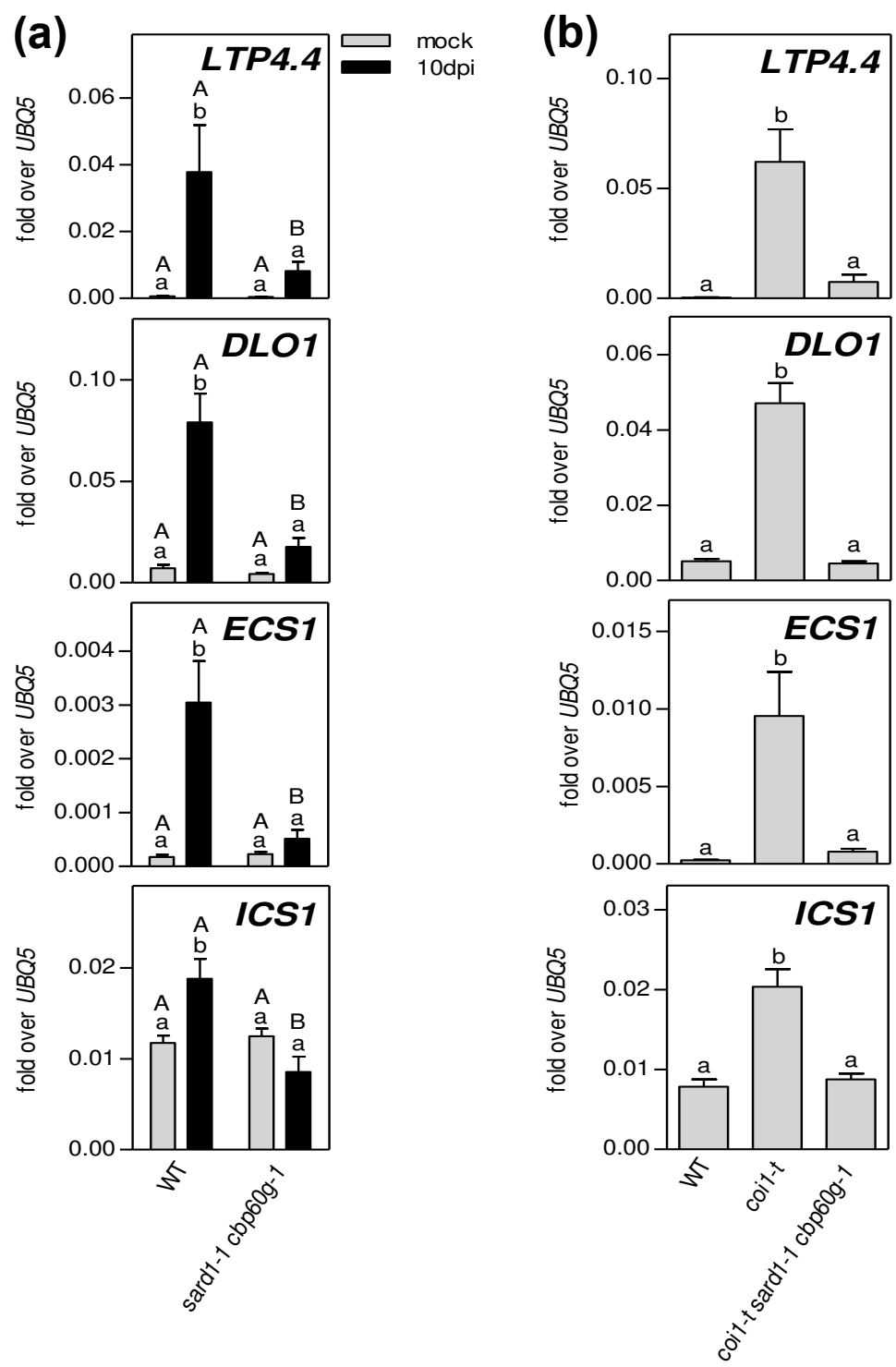

Figure 6. SARD1/CBP60g are responsible for induction of genes of interest after infection with $V$. longisporum and for basally upregulated gene expression in coi1.

(a) LTP4.4, DLO1, ECS1 and ICS1 transcript levels, measured by qRT-PCR. RNA was extracted from roots 10 days after mock treatment or infection with $1 \times 10^{6}$ spores $/ \mathrm{mL} \mathrm{V}$. longisporum. Bars are means \pm SEM of thirteen to sixteen roots per genotype. For statistical analysis, a two-way ANOVA was performed followed by Bonferroni's multiple comparison test; lowercase letters denote significant differences within each genotype between mock and 10 dpi $(p<0.05)$, uppercase letters denote significant differences between genotypes subjected to the same treatment $(p<0.05)$. (b) LTP4.4, DLO1, ECS1 and ICS1 transcript levels, measured by qRT-PCR. RNA was extracted from roots 10 days after mock treatment. Bars are means \pm SEM of eight roots per genotype. For statistical analysis, a oneway ANOVA was performed followed by Tukey's multiple comparison test; lowercase letters denote significant differences between samples $(p<0.05)$. 


\section{SARD1 is required but not sufficient for induction of genes of interest}

Having identified SARD1/CBP60g as main regulators of our genes of interest, we aimed to create SARD1 overexpression lines to see if we could induce expression of our target genes in such plants. Plants overexpressing SARD1 in roots should mimic enhanced gene expression levels that we see after infection with $V$. longisporum and basally in coi1. To this end, we constructed a SARD1 clone using the genomic sequence from the transcriptional start site with a C-terminal three times HA and Strep-II tag under control of the UBIQUITIN10 (UBQ10) promoter. Zhang et al. (2010) reported increased SA levels and a dwarfed phenotype of their SARD1 overexpression lines (SARD1 OXS) under control of the endogenous SARD1 promoter. The underlying reason for this is likely the activation of ICS1 by SARD1, activating SA defence signalling. As we wanted to avoid growth defects, we not only transformed our SARD1 construct into Col-0 plants but also into sid2 mutants. Overexpressing SARD1 in the sid2 background avoids triggering ICS1-derived SA production and its possible negative effects on plant growth. SARD1 overexpression in Col-0 lead to about 2-fold higher induction of SARD1 compared to $V$. longisporum-infected WT plants (Figure S9a) and thus is approximately comparable to $S A R D 1$ transcript levels in coi1 roots (Figure $3 \mathrm{~d}$ ). In the end, our overexpression line in the Col-0 background only showed mild defects in rosette size compared to the empty vector control (Figure S9b). As expected, overexpression of SARD1 had no effect on rosette size in the sid2 background.

To our surprise, we found that overexpressing SARD1 in roots in either Col-0 or sid2 led to weak to no induction of target genes (Figure 7a). LTP4.4 showed no induction in Col-0 or sid2 background. DLO1 and ECS1 showed approximately 2-fold induction in SARD1 OXs in Col-0 but no induction in the lines in sid2 background. ICS1 was not at all induced in the SARD1 overexpression line in Col-0.

In contrast, expression of our target genes in shoots of the exact same plants showed much stronger gene induction (Figure 7b). LTP4.4 was 25-fold and 11-fold induced in Col-0 and sid2, 
(a)

ROOTS
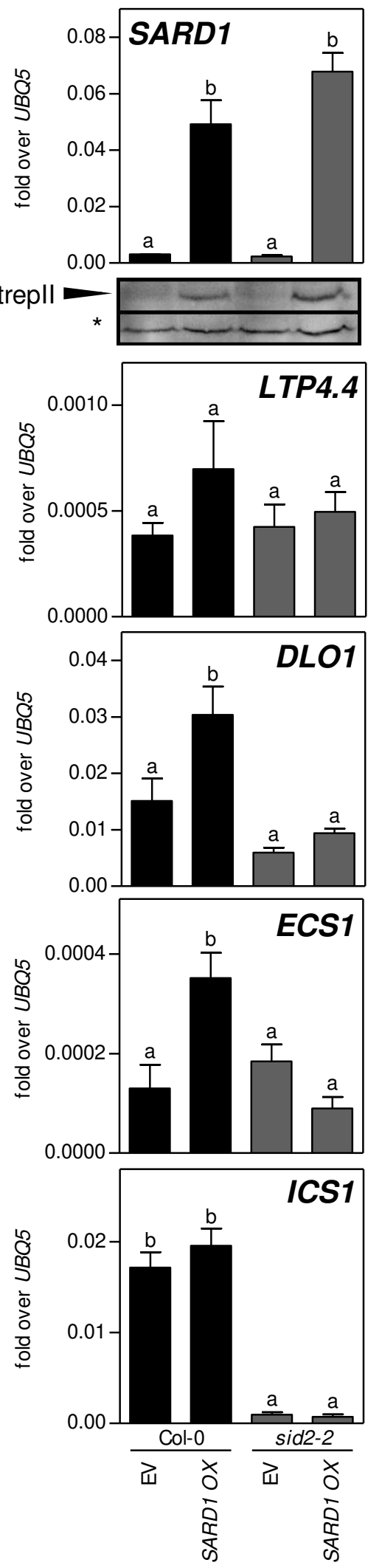

(b) SHOOTS
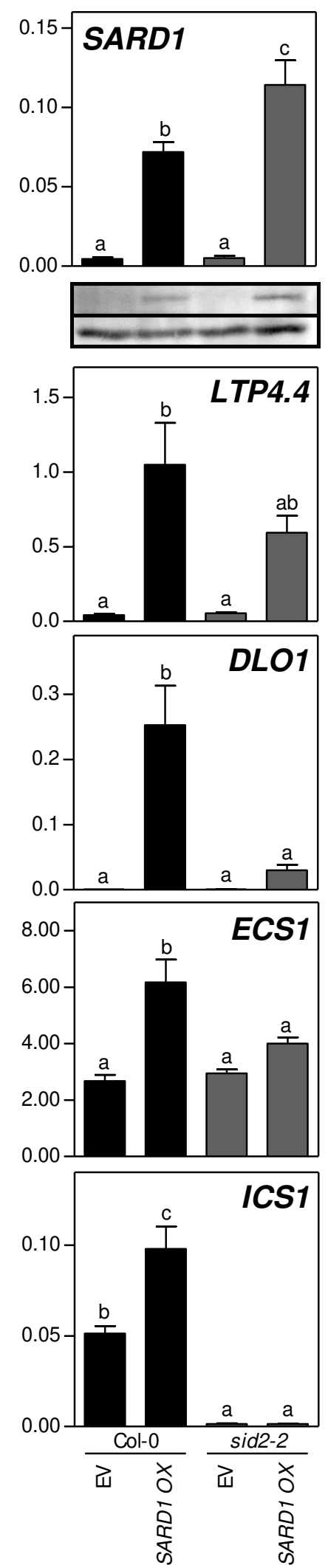

Figure 7. Overexpression of SARD1 in roots does not lead to strong target gene activation. Full legend on next page 
Figure 7. Overexpression of SARD1 in roots does not lead to strong target gene activation.

SARD1, LTP4.4, DLO1, ECS1 and ICS1 transcript levels, measured by qRT-PCR. RNA was extracted from (a) roots or (b) shoots 10 days after mock treatment of SARD1 overexpression lines (SARD1 OX) and empty vector (EV) controls in both Col-0 (black bars) and sid2-2 (gray bars) background. Bars are means \pm SEM of three to six roots or shoots per line. For statistical analysis, a one-way ANOVA was performed followed by Tukey's multiple comparison test; lowercase letters denote significant differences between samples $(p<0.05)$.

Insert: Western blot of protein extracts obtained from roots and shoots of SARD1 overexpression lines (SARD1 OX) and empty vector (EV) controls in Col-0 and sid2-2 background. Per lane, six roots or three shoots were pooled from each line. C-terminally 3xHA-Strepll tagged SARD1 protein levels were detected using an anti-HA antibody. * depicts an unspecific band shown as loading control.

respectively. DLO1 was 372 -fold induced in Col-0 plants, when it was only 2 -fold induced in roots in the same genotype. In sid2, DLO1 was 33-fold induced, albeit non-significantly in statistical analysis. ECS1 showed no stronger induction in shoots as compared to roots, only stronger expression overall. In shoots, ICS1 was also induced twofold.

We also tested expression of WRKY46 and WRKY54 in the SARD1 OX lines (Figures S10a and S10b). WRKY46 showed a similar induction pattern to LTP4.4, with SARD1 OX lines only inducing gene expression in shoots. For WRKY54, lower expression in sid2 compared to Col0 can be seen in both EV and SARD1 OX plants. WRKY54 shows weak induction in the Col0 SARD1 OX line in roots and slightly stronger induction in shoots, again with strong differences in Col-0 and sid2.

Overall, in roots, SARD1 alone is not sufficient to induce gene expression to a scale that was observed in infected roots or in coi1 roots. In contrast to roots, SARD1 can induce expression of target genes strongly in shoots, where COl1 does not act as a repressor of these genes (Figure 4). The only exception is ECS1 which is similarly expressed in SARD1 OXs in roots and shoots. Possibly, SARD1 OXs do not show strong target gene induction in roots because SARD1 is unable to overcome the COl1-mediated repression of these genes. This evidence is in favour of our hypothesis, that COl1 mediated repression is overcome by infection with $V$. longisporum. Only after the negative effect of COI1 on these promoters is lifted, SARD1 is able to activate gene expression. 


\section{Basal upregulation of SARD1-controlled defence gene expression in roots is not responsible for the tolerance of coi1 plants against $V$. longisporum}

Our main interest is the regulation of the 149 genes de-repressed in coi1 roots and induced after infection with $V$. longisporum. We anticipated that understanding their regulation may provide insights into the mechanism through which COI1-mediated repression works and how it may be inactivated. Nevertheless, having identified SARD1/CBP60g as the master regulators of this set of genes, we were now also able to address the question whether the upregulation of these genes in coi1 roots is the underlying reason for the tolerance of coi1 plants against $V$. longisporum.

Grafting experiments had shown that plants with coi1 root stock and WT shoots were tolerant against fungal infection (Ralhan et al., 2012). Hence, coi1 roots and not shoots are important for tolerance. This seemingly contradicts the fact that fungal entry into coi1 roots is not hindered and that infection is only contained by reduced proliferation of $V$. longisporum in shoots of coi1 plants (Ralhan et al., 2012). The genes we are investigating here are induced after infection and GO term analysis identified them to be defence-related (Figure 2). Some of these genes, like LTP4.4, are secreted to the apoplast (McLaughlin et al., 2015), others have a mobile mRNA, like the call wall protein ECS1 (Thieme et al., 2015). It is possible that products of these genes, constitutively made in the root, are transported up into the shoot where they accumulate. Once the fungus reaches the shoot, its proliferation is impaired by the defence compounds that are deposited there.

With this idea in mind, we again used mutations in SARD1 and CBP60g to examine the phenotype of infected plants unable to activate our genes of interest. Initially, we compared the phenotype of sard1 cbp60g plants against WT plants to see if the mutants would be hypersusceptible to infection with $V$. longisporum. Even at $21 \mathrm{dpi}$ we could not determine any difference between sard1 cbp60g and WT plants (Figure S11). To better address the question if the constant activation of these genes in coi1 roots is the reason for the tolerance, we infected coi1 sard1 cbp60g plants. We have already shown that in these roots target gene activity is reduced to WT levels again in the coi1 background (Figure $6 \mathrm{~b}$ ). coi1 plants only showed $11 \%$ 
loss of leaf area at $15 \mathrm{dpi}$, whereas WT plants showed much greater susceptibility to $V$. longisporum with $46 \%$ loss of leaf area (Figure 8). With $21 \%$, the coi1 sard1 cbp60g plants only showed a slightly increased and non-significant loss of leaf area compared to coi1 plants. Hence, the upregulation of the group of 149 SARD1/CBP60g-regulated defence genes in coi1 roots has only a very slight effect towards tolerance and is not the overall reason for the tolerance against $V$. longisporum.

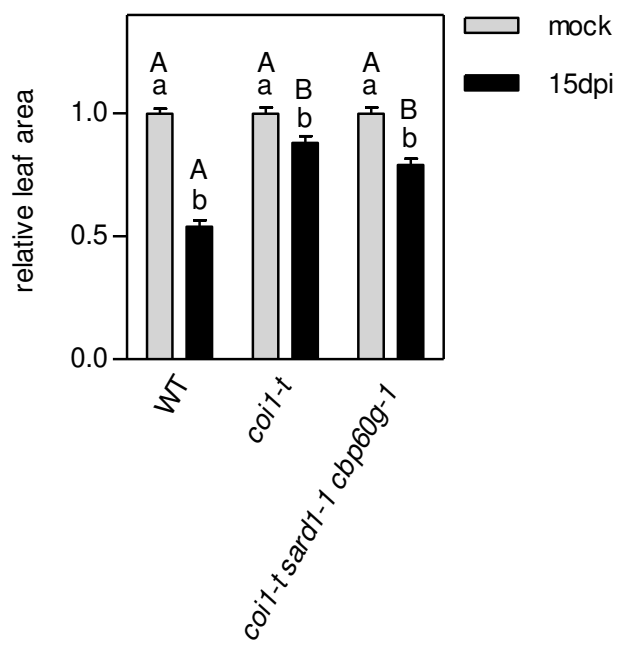

Figure 8. The SARD1/CBP60g-controlled genes upregulated in coi1 roots are not responsible for the tolerance of coi1 plants against infection with V. longisporum.

Relative leaf area of plants 15 days after mock treatment or infection with $5 \times 10^{5}$ spores $/ \mathrm{mL}$ sGFPexpressing $V$. longisporum. Bars are means \pm SEM of a total of 47-48 plants per treatment from three independent experiments with 15-16 plants per treatment each. For statistical analysis, a two-way ANOVA was performed followed by Bonferroni's multiple comparison test; lowercase letters denote significant differences within each genotype between mock and $15 \mathrm{dpi}(p<0.05)$, uppercase letters denote significant differences between genotypes subjected to the same treatment $(p<0.05)$.

\section{Discussion}

In this manuscript, we report the identification of genes that are differentially expressed in roots of $V$. longisporum-infected Arabidopsis plants at $10 \mathrm{dpi}$. Induction of the large majority of genes did not require biosynthesis of the major defence hormones SA and JA. Apart from genes associated with cell wall remodelling, a set of genes associated with the SA-dependent shoot 
defence response systemic acquired resistance were induced even in the SA biosynthesis mutant sid2. These genes were already up-regulated in mock-treated coi1 plants, suggesting that inactivation of COl1 by a yet unknown signal might be responsible for their SAindependent induction.

\section{Induction of the majority of Verticillium longisporum-induced genes is independent of de novo synthesis of jasmonic acid or salicylic acid}

Transcriptome analysis of Verticillium longisporum-infected Arabidopsis roots has been performed before with axenically grown seedlings (Iven et al., 2012; Ulrich et al., 2021). In our hands, $V$. longisporum only entered the xylem when seedlings were cultured for one day without any nutrients. Under these conditions, we did not observe any changes at the transcriptional level at 4 dpi. In contrast, Verticillium-induced gene expression was observed when seedlings continued to be cultivated on MS plates (Iven et al., 2012). Under these conditions, the fungus was able to colonize the cortex, but entry into the xylem was not documented. Here, we harvested tissue from plants that had been cultivated on soil for $10 \mathrm{dpi}$. At this stage, microscopical data is hampered by strong autofluorescence of the root system. We detected GFP-tagged fungal hyphae only occasionally in a few roots (Ralhan et al., 2012). In contrast to the infection system on MS plates, the root surface was not covered with fungal mycelium. In WT plants segregating from the sterile aos and coi1 plants, we observed that 881 and 948 genes were induced, respectively. Although both wild-types should react in the same manner to $V$. longisporum, only 772 genes were induced in both lines. Most of the genes that do not fall into this group barely missed the threshold in one of the wild-types. GO term enrichment analysis unravelled preferential up-regulation of cell wall remodelling genes, with VND7 coding for a master transcriptional regulator of de novo xylem formation being strongly induced. In contrast, Iven et al. (2012) had observed enrichment of genes involved in tryptophane biosynthesis and tryptophane-derived secondary metabolism. Marker genes of this pathway like CYP79b2 and CYP79b3 were not induced under our conditions. Since these genes were induced even at 8 dpi in the axenic infection system (Iven et al., 2012), we do not 
think that the difference in the time point of analysis is responsible for distinct gene expression patterns. We rather imagine that growth conditions and/or localisation of the fungus within or around the root affect the outcome of the transcriptional response. The response of aos and sid2 were to the same degree different as the two wild-types indicating that increased levels of SA and JA do not play a major role for the induction. This notion was already evident from the principal component analysis and is supported by the fact, that key biosynthesis enzymes of the pathways like ICS1, AOS or OPDA REDUCTASE 3 (OPR3) were not induced.

\section{A portion of Verticillium-inducible genes is constitutively de-repressed in coi1}

Principal component analysis revealed that the coi1 transcriptome showed the largest difference to the transcriptomes of the other genotypes, both in the mock-treated and the fungal-infected samples. This difference is mainly due to the constitutive up-regulation of 316 genes in coi1 roots. This repressive effect of COI1, which is only observed in roots and not in shoots, has been detected before under axenic growth conditions (Ulrich et al., 2021).

Roughly half of these genes are induced upon infection of soil-grown plants with $V$. longisporum. Notably, constitutive expression of these genes in coi1 is not further enhanced by $V$. longisporum. This raises the hypothesis that - with regard to transcriptional activation of these genes - infection cannot be sensed without COl1. A possible scenario is that the repressive function of COl1 is inactivated upon infection. This postulated inactivation is almost as efficient as the genetic inactivation, suggesting that it occurs systemically and not only in a few locally infected cells. We currently have no information on the spatial distribution of root gene activation after $V$. longisporum infection, but it is unlikely that all COl1-expressing cells are in direct contact with the fungus. Therefore, we favour the idea that $V$. longisporum infection leads to systemic inactivation of at least those genes that are suppressed by COl1.

To explain systemic effects in the root system, we have to postulate a signal generated in locally infected roots that travels to the shoot, where a second signal moving back to the root system is generated (Figure 9). Primary signals travelling from the root to the shoot might either 
be synthesized by the fungus or the plant. It is known that plant-derived small peptides can be transported to the shoot where they can diffuse into the phloem. Their perception leads to the generation of other signalling molecules that are transported down to all parts of the root via the phloem. Examples for this mechanism have been described before in the context of nitrogen starvation, where a small peptide (CEP) is generated in N-starved roots (Tabata et al., 2014). This peptide is recognized by a receptor (CEPR) in the shoot, leading to the synthesis of a glutaredoxin-like small protein (CEPD) which promotes gene expression leading to increased nitrate uptake (Tabata et al., 2014; Ohkubo et al., 2017). Indeed, expression of the 79 amino acid-long peptide CLE1 is highly induced upon infection in all five genotypes. Alternatively, root volatiles or small RNAs might serve as mobile signals (Godard et al., 2008; Tsikou et al., 2018; Okuma et al., 2020).

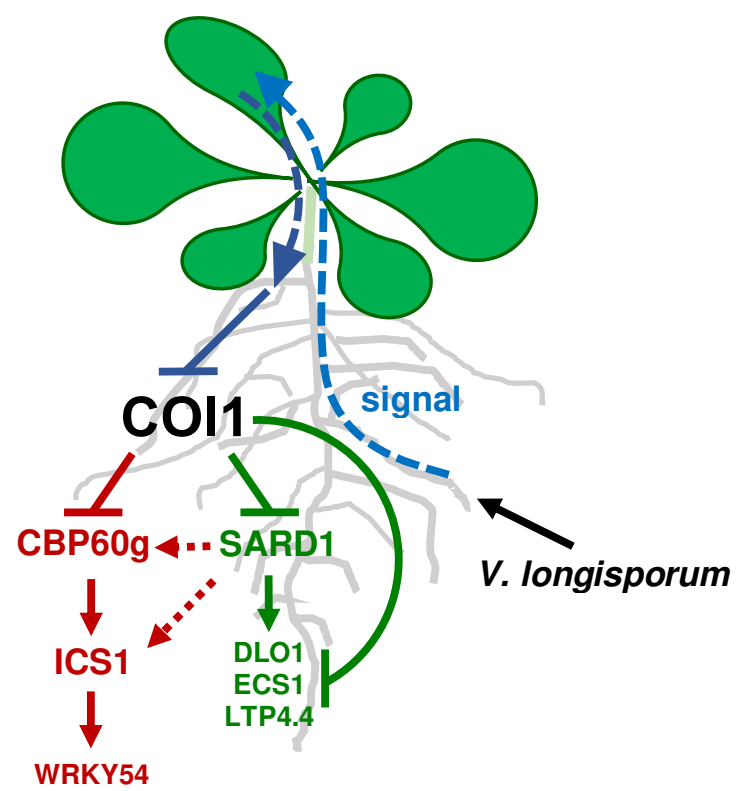

Figure 9. After V. longisporum infection, inactivation of COl1 leads to activation of the SARD1branch but not the CBP60g-branch of downstream defence gene activation.

Model where $V$. longisporum infection generates a root to shoot signal (light blue) that leads to the systemic inactivation of COI1's repressive function in roots (dark blue). Inactivation of COI1 leads to activation of SARD1 and its downstream targets (green pathway) but inactivation of COl1 after $V$. longisporum infection is not sufficient to induce the CBP60g-regulated ICS1-branch (red pathway). COI1 also has a direct repressive effect on SARD1-target genes like DLO1, ECS1 and LTP4.4 which is lifted after infection (green pathway). 


\section{SARD1/CBP60g are the master regulators of COl1-supressed genes}

Motif mapper analysis identified the 'GAAATTT' motif as being enriched particularly in the group of promoters that is repressed by COI1 and induced by $V$. longisporum. The 'GAAATTT' motif is recognised by the partially redundant transcription factors CBP60g and SARD1. In shoots, these proteins are required for activation of SA production after pathogen infection, for the activation of SA-dependent signalling and for the activation of SA-independent immune responses (Wang et al., 2009, 2011; Zhang et al., 2010; Sun et al., 2015). Consistent with the GO term-based findings that our COl1-repressed genes are functionally connected to SArelated defence responses and consistent with repression of $S A R D 1 / C B P 60 g$ transcription by COI1, we hypothesise that SARD1/CBP60g might play an important role for the expression of COI1-repressed genes. By analysis of the coi1 sard1 cbp60g triple mutant, we found that genes upregulated in coi1 (LTP4.4, ECS1, DLO1, ICS1, WRKY46 and WRKY54) indeed require SARD1 (Figures $6 a$ and S8a). At least in shoots, direct in vivo binding of SARD1 to the promoter regions of LTP4.4, ECS1 and ICS1 has been demonstrated by chromatin immunoprecipitation experiments (Sun et al., 2015). LTP4.4 and ECS1 are de-repressed in the coi1 sid2 mutant, supporting the idea that they are directly regulated by SARD1 and not indirectly through SARD1-mediated activation of SA synthesis (Figure S5c). The expression pattern of WRKY46 was similar to LTP4.4 and ECS1 in coi1 sid2 and coi1 sard1 cbp60g, even though it was not in the group of 149 genes but in the 167 genes not induced after infection (Figures S8a and S8b). Expression in coi1 sard1 cbp60g roots shows that it is not as strongly dependent on SARD1 as the others. As the group of the 167 genes contains all those that are not significantly induced at 10 dpi under control of COI1, it might also contain more SARD1dependent genes that have missed the set threshold for significant induction in our data analysis. The upstream regions of DLO1 and WRKY54 are not bound by SARD1 in shoots. The expression of these genes is reduced in coil sid2, indicating that they are predominantly regulated by SARD1-activated SA synthesis. However, there is also at least one exception, namely AT5G39120, which is activated through a different mechanism. 
Our loss of function analysis suggests that enhanced expression of SARD1 in coi1 might be the reason for enhanced expression of downstream genes. However, constitutive expression of SARD1 in Col-0 background did not activate downstream genes to the same extent as enhanced expression of $S A R D 1$ in coi1. This is different from the situation in shoots, where SARD1 can have a clear activating effect even in the absence of ICS1-derived SA. It can be envisioned that COI1 interferes with SARD1 activity at downstream genes or that - in roots SARD1 action requires additional proteins that are de-repressed in coi1.

\section{ICS1 is not efficiently induced after Verticillium longisporum infection}

In shoots, SARD1 binds to the promoters of the two SA biosynthesis enzymes ICS1 and PBS3, resulting in their transcriptional activation and subsequent SA synthesis (Sun et al., 2015). In coi1 roots, ICS1 and PBS3 expression is also enhanced in a manner that requires SARD1 (shown for ICS1) leading to the activation of SA-inducible genes like WKRY54. However, upon V. longisporum infection, only PBS3, but not ICS1, is induced (Table 1 and subtables). Our first idea was that $V$. longisporum might encode a specific effector that interferes with ICS1 expression. However, since we consider systemic induction as being likely, we propose that other mechanisms might be responsible for the divergent regulation of ICS1 and PBS3. In shoots, ICS1, PBS3, SARD1 and CBP60g are highly co-expressed. In roots, we observe a divergent expression pattern with $P B S 3$ and $S A R D 1$ being up-regulated in coi1 and being

induced by $V$. longisporum. In contrast, CBP60g and ICS1 are up-regulated in coi1 but not induced by $V$. longisporum. Transcription of CBP60g is less than 2-fold enhanced in coi1. Assuming a more prominent role for CBP60g in inducing ICS1, it can be envisioned that inactivation of COI1 by $V$. longisporum might not yield sufficient amounts of CBP60g to support expression of ICS1 (Figure 9). Another possible scenario would be that COI1 is only inactivated at specific promoters or that is universally inactivated but other repressors take over on specific promoters as a second line of regulation.

When first reporting the repressive function of COI1 on SA-related genes in roots, we speculated that inappropriate up-regulation of this pathway might interfere with the composition 
of the microbiome in the rhizosphere, making an extra layer of repression necessary. Indeed, it has been shown that beneficials colonise roots faster and better when root SA-defences are blocked or impaired (Herrera Medina et al., 2003; Martínez-Medina et al., 2017). Likewise, elevated SA levels delay and reduce colonisation by beneficials (Martínez-Abarca et al., 1998; Herrera Medina et al., 2003; Martínez-Medina et al., 2017). COl1-mediated gene repression might serve as a safety stop to allow the onset of a strong defence response only when necessary. To lift this repression after colonisation of the root with a pathogen, a novel induction mechanism had to be developed. Our findings that this is not relevant for the interaction with $V$. longisporum does not rule out the option that this programme might be efficient against other biotrophic root pathogens.

\section{Materials and Methods}

\section{Plant Material}

All plants used in this article are Arabidopsis thaliana Col-0 background. Genotypes used in the study, corresponding references and sources are: aos (SALK_017756) from Nottingham Arabidopsis Stock Centre (NASC); coi1-1 (Xie et al., 1998) from John Turner (University of East Anglia, Norwich, UK); coi1-t (SALK_035548) (Mosblech et al., 2011) from Ingo Heilmann (Martin-Luther-University, Halle, Germany); coi1-1 sid2-2 (Ulrich et al., 2021); sard1-1 cbp60g1 (Zhang et al., 2010) from Yuelin Zhang (UBC Vancouver, Canada); sid2-2 (Wildermuth et al., 2001) from Frederick M. Ausubel (Harvard University, Boston, USA). The coi1-t sard1-1 cbp60g-1 triple mutant was generated through crossing of the respective above-mentioned genotypes. Primers for genotyping are listed in Table S1.

\section{Plant Growth Conditions and Treatments}

Surface sterilised seeds were sown onto Murashige-Skoog-medium (MS) supplemented with $2 \%$ Sucrose and kept at $4^{\circ} \mathrm{C}$ for $24-72$ hours in darkness. Plates were placed horizontally into 
growth chambers with short day conditions (8-h-day/16-h-night cycle, $22^{\circ} \mathrm{C} / 22^{\circ} \mathrm{C}, 60 \%$ humidity) with a photon flux density of $80-100 \mu \mathrm{mol} \mathrm{m} \mathrm{m}^{-2} \mathrm{~s}^{-1}$. After 14 days plants were carefully transferred onto a 1:1 mix of sand (white, 1-2 mm grain size, Rosnerski, Königslutter, Germany) and twice steamed soil (Fruhstorfer Erde, Spezial Substrat, Typ T, Str. 1 fein, HAWITA, Vechta, Germany) on a thin layer of Seramis (Westland Deutschland, Mogendorf, Germany). The mixture was initially watered with $0.1 \%$ Wuxal Super (Manna, AmmerbuchPfäffingen, Germany) in $\mathrm{dH}_{2} \mathrm{O}$. Plants were grown on the sand-soil mixture for another 14 days under above-mentioned short-day conditions with increased photon flux density of 120-140 $\mu \mathrm{mol} \mathrm{m}^{-2} \mathrm{~s}^{-1}$. For the first seven days plants were covered with a transparent hood, on day seven the hood was opened and on day nine it was fully removed. If genotyping was required, a single leaf was clipped from each plant during the first week of growth on the sand-soil mixture. Subsequently, plants were carefully uprooted from the sand-soil mixture and their roots washed in tap water. Roots were then dipped in tap water as mock treatment or $V$. longisporum spore suspension for 45 minutes. Afterwards plants were planted into individual pots containing twice steamed soil (Fruhstorfer Erde, Spezial Substrat, Typ T, Str. 1 fein, HAWITA, Vechta, Germany) soaked with $0.2 \%$ Wuxal Super, where plants were kept for a final 10 to 21 days in short day conditions at $120-140 \mu \mathrm{mol}$ photons $\mathrm{m}^{-2} \mathrm{~s}^{-1}$. During the first two days on soil pots, plants were kept under transparent hoods. A rootstock or shoot of one single plant was harvested for one biological replicate if not otherwise specified.

For nlp14 infiltration in Figure 5b plants were grown in single pots for four and a half weeks in 12-h-day/12-h-night cycle, $22^{\circ} \mathrm{C} / 22^{\circ} \mathrm{C}, 60 \%$ humidity. nlp14 (GVYAIMYSWYFPKD; GenScript, Leiden, Netherlands) was solved in $100 \%$ DMSO and aliquots were stored at $10 \mathrm{mM}$ in $-70^{\circ} \mathrm{C}$. Using a needleless syringe, three leaves of similar age from each plant were infiltrated with $1 \mu \mathrm{M}$ nlp14 in Millipore $\mathrm{H}_{2} \mathrm{O}$ with $0.01 \%$ DMSO or just with Millipore $\mathrm{H}_{2} \mathrm{O}$ with $0.01 \%$ DMSO as mock treatment and harvested after $24 \mathrm{~h}$.

For root treatment with $1 \mathrm{mM} \mathrm{SA}$ (Figure S6), plants were grown as specified above. After growth on sand-soil mixture, plants were carefully uprooted and their roots dipped into tap 
water for mock treatment or $1 \mathrm{mM}$ sodium salicylate solution (Sigma-Aldrich/Merck KGaA, Darmstadt, Germany) for 24 hours.

For phenotypic analysis of plants in Figure 8, heterozygous seed batches of coi1-t and coi1-t sard1-1 cbp60g-1 (homozygous for sard1-1 cbp60g-1 but heterozygous for coi1-t) were initially placed on MS medium supplemented with $2 \%$ Sucrose and $50 \mu \mathrm{M}$ methyl jasmonate to identify plants homozygous for the coi1-t mutation (Feys et al., 1994). Consecutive treatment was the same as described above.

\section{Fugal culture and inoculation}

Verticillium longisporum isolate VI43 (Zeise and Von Tiedemann, 2002) provided by Daguang Cai (Christian-Albrechts-University, Kiel, Germany) and Verticillium longisporum VI43 sGFP (Eynck et al., 2007) provided by Andreas von Tiedemann (Georg-August University, Goettingen, Germany) conidia stocks were stored in $21.5 \%$ glycerol at $-70^{\circ} \mathrm{C}$. For preparation of conidia batches for plant inoculation, stock conidia from glycerol were cultivated in liquid simulated xylem medium (SXM) (Hollensteiner et al., 2017), supplemented with $275 \mathrm{mg} / \mathrm{L}$ Cefotaxim, for 7 days in a rotary shaker at $23^{\circ} \mathrm{C}$ and $90 \mathrm{rpm}$. Conidia were harvested by filtering through a fluted filter (Nucleo Bond folded filters, Macherey-Nagel, Düren, Germany), washed in sterile tap water and their concentration determined with a hemocytometer. Glycerol was added to a final concentration of $21.5 \%$. The conidia infection stocks were initially stored in $20^{\circ} \mathrm{C}$ for 5 days and subsequently stored at $-70^{\circ} \mathrm{C}$ until the day of inoculation. On inoculation day conidia stocks were thawed, centrifuged for 8 mins at $8000 \mathrm{rpm}$ and resuspended in tap water to a final concentration of $5 \times 10^{5}$ or $1 \times 10^{6} \mathrm{spores} / \mathrm{mL}$ for plant inoculation.

\section{Leaf Area Measurement}


For disease phenotype analysis, photographs of individual plants were taken at 15 or $21 \mathrm{dpi}$. The surface area of the whole rosette was determined with the 'BlattFlaeche' Software (Datinf GmbH, Tübingen, Germany) (Ralhan et al., 2012).

\section{RNA-seq Analysis}

coi1-t plants are male sterile whereas fertility of the aos mutant can be rescued by methyl jasmonate (MeJA) treatment. As we wanted to avoid differences in the history of the seed batches by rescuing aos mutants with MeJA treatment, we again used heterozygous populations of coi1-t and aos plants that were genotyped with primers specified in Table S1 (Ulrich et al., 2021). For RNA-Seq analysis, twelve single homozygous roots of either aos, $\mathrm{WT}_{\text {aos, }}$ coi1-t, $\mathrm{WT}_{\text {coit-t }}$ or sid2-2 were combined for one replicate; replicates per genotype and treatment were obtained from four independent infection experiments. RNA was extracted using the Trizol method (Chomczynski and Mackey, 1995) and RNA quality was controlled with an AGILENT BIOANALYZER 2100. Single-end 50-bp raw reads from mRNA sequencing were generated with the Illumina HiSeq 2000 platform and sequence images were transformed with the Illumina BaseCaller software to BCL files, which were subsequently demultiplexed to FASTQ files with CASAVA (v1.8.2). Using a Galaxy platform (Afgan et al., 2018), mapping of reads to the Arabidopsis thaliana genome reference sequence (TAIR10 release-39, ftp://ftp.ensemblgenomes.org/pub/plants/release-39) was carried out with RNA STAR (Galaxy version 2.5.2b-2 (Afgan et al., 2018) and aligned reads were quantified using HTSeq-count (Galaxy version 0.9.1 (Afgan et al., 2018)). Normalization and differential expression analysis was performed with DESeq2 (Galaxy version 2.11.40.6+galaxy1 (Love et al., 2014)) to obtain log2-fold changes and adjusted $p$ values (Benjamini-Hochberg-corrected).

For Gene Ontology (GO) term enrichment analysis, the agriGO v2.0 program was used (Tian et al., 2017). Categories > 5-fold enrichment against the Arabidopsis genome are shown in Figures 2, S2 and S3. 


\section{Quantitative Reverse Transcription (qRT)-PCR}

RNA extraction, cDNA synthesis and qRT-PCR were performed as previously described (Ulrich et al., 2021). Calculations were done according to the $2^{-\Delta C T}$ method (Livak and Schmittgen, 2001) using the UBQ5 (AT3G62250) or PP2A (At1G13320) transcripts as a reference. Primers used for qRT-PCR are listed in Table S2.

\section{Statistical Analysis}

GraphPad Prism 5.0 (GraphPad Software, Inc., San Diego, CA) was used to conduct statistical analysis.

\section{Motif Mapper Analysis}

Analysis of cis element enrichment was done using the Cluster Analysis Real Randomization algorithm incorporated into the Motif Mapper Version 5.2.4.0 (Berendzen et al., 2012). By comparison to 1000 randomly composed, equally sized, reference promoter datasets, significant distribution alterations were defined as described in (Zander et al., 2014).

\section{Generation of Transgenic Plants and Western Blot Analysis}

Recombinant SARD1 plasmids were created via GATEWAY cloning (Invitrogen, Karlsruhe, Germany). The genomic sequence of SARD1 was amplified from the annotated transcriptional start site to the last amino acid of the coding region, using primers SARD1GWfwd and SARD1noStopGWrev (Table S3). The primers added GATEWAY recombination sites to the PCR product, which were used to introduce it into pDONR207. From there, SARD1 was further introduced into pUBQ10GW3HAstrepll7 (Budimir et al., 2021) adding a three times HA and Strepll C-terminal tag. The final plasmid pUBQ10-SARD1-3HAstrepll7 was introduced into Col-0 and sid2-2 plants via Agrobacterium tumefaciens-mediated gene transfer (Clough and Bent, 1998). As empty vector (EV) controls the original plasmid pUBQ10GW3HAstrepll7 was 
transformed into plants. Transgenic plants were characterised via BASTA (Bayer CropScience AG, Monheim, Germany) selection and Western Blot analysis was used to assess SARD13xHA-Strepll protein levels in homozygous plants.

For Western Blot analysis, $250 \mu \mathrm{L}$ extraction buffer (4M urea, $16.6 \%$ glycerol, $5 \%$ SDS, $5 \% \beta$ mercaptoethanol) was added per $100 \mathrm{mg}$ ground root or shoot material. The Pierce $660 \mathrm{~nm}$ assay kit (Thermo Scientific, Rockford, IL USA) was used to determine protein concentrations of extracts. $50 \mu \mathrm{g}$ of root or shoot protein were loaded and separated on a $10 \%$ SDS gel. Transfer of proteins to a polyvinylidene difluoride membrane was done via semi-dry electroblotting. Proteins were detected using an aHA-antibody (Abcam, Cambridge, United Kingdom) and Super SignalTM West Femto Maximum Sensitivity Substrate (Thermo Scientific, Rockford, IL 1606, USA).

\section{SA Measurements}

Measurements of salicylic acid in root material were kindly carried out by Krzysztof Zienkiewicz at the Department of Biochemistry, Albrecht-von-Haller Institute for Plant Sciences, GeorgAugust University, Göttingen, Germany. Nanoelectrospray (nanoESI) analysis was carried out as previously described (Kusch et al., 2019). After reversed phase separation of constituents by an ACQUITY UPLC® system (Waters Corp., Milford, MA, USA) equipped with an ACQUITY

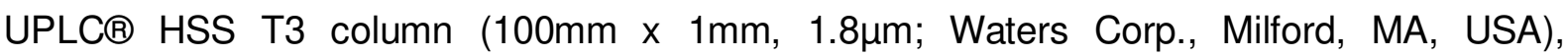
phytohormones were ionized in a negative mode and determined in a scheduled multiple reaction monitoring mode with an $A B$ Sciex $4000 Q T R A P \circledast$ tandem mass spectrometer $(A B$ Sciex, Framingham, MA, USA). Mass transitions were as described previously (Iven et al., 2012).

\section{Accession numbers}


Sequence data from this article can be found in The Arabidopsis Information Resource (http://www.arabidopsis.org/) under the following accession numbers: ANAC076 (AT4G36160), AOS (AT5G42650), CBP60G (AT5G26920), COI1 (AT2G39940), DLO1 (AT4G10500), ECS1 (AT1G31580), ERF54 (AT4G28140), ICS1 (AT1G74710), LTP4.4 (AT5G55450), PP2A (At1g13320), RmIC-like cupins superfamily protein (AT5G39120), SARD1 (AT1G73805), UBQ5 (AT3G62250), UBQ10 (AT4G05320), WRKY46 (AT2G46400), WRKY54 (AT2G40750).

\section{Funding}

Work for this article was funded by the Deutsche Forschungsgemeinschaft (GA330/24).

\section{Author contributions}

L.U. and C.G. wrote the manuscript; L.U. designed the experiments, acquired and analysed most of the data; C.G. designed and supervised the research; J.S. performed experiments for the RNA-seq; C.T. designed and supervised the research and analysed the RNA-seq data.

\section{Acknowledgements}

We thank Anna Hermann, Katharina Dworak and Ronald Scholz for excellent technical assistance and Natalie Leutert for help with characterisation of transgenic SARD1 OX lines. We also thank the Transcriptome and Genome Analysis Laboratory (TAL) at the University Medical Center Göttingen (UMG) for performing the RNA-seq analysis.

\section{Supplementary Information}

Table S1. Primers for Genotyping

Table S2. Primers for qRT-PCR

Table S3. Primers for Cloning 
Figure S1. Gene induction patterns are largely overlapping in aos, $\mathrm{WT}_{\text {aos }}, \mathrm{WT}_{\text {coit- } t}$, and sid22 at $10 \mathrm{dpi}$.

Figure S2. Gene Ontology (GO) term enrichment analysis of the 772 genes significantly induced in $\mathrm{WT}_{\text {aos }}$ and $\mathrm{WT}_{\text {coit-t }}$ at $10 \mathrm{dpi}(>2$-fold; $p<0.05)$.

Figure S3. Gene Ontology (GO) term enrichment analysis of (a) the 167 genes upregulated in mock-treated coi1-t compared to mock-treated aos, $\mathrm{WT}_{\text {aos }}, \mathrm{WT}_{\text {coit-t }}$ and sid2-2 (> 2-fold; $p<$ 0.05) and not significantly induced in aos, $\mathrm{WT}_{\text {aos }}, \mathrm{WT}_{\text {coit-t }}$ and sid2-2 at $10 \mathrm{dpi}$ and (b) the 512 genes induced in aos, $\mathrm{WT}_{\text {aos }}, \mathrm{WT}_{\text {coit-t }}$ and sid2-2 at $10 \mathrm{dpi}(>2$-fold; $p<0.05)$ but not upregulated in mock-treated coi1-t compared to mock-treated aos, $\mathrm{WT}_{\text {aos }}, \mathrm{WT}_{\text {coi1-t }}$ and sid2-2. Figure S4. Infection with $V$. longisporum does not lead to accumulation of SA in roots.

Figure S5. In coi1 roots, basally elevated marker gene expression is independent of elevated SA levels in most cases.

Figure S6. Genes of interest can mostly be induced by SA in roots.

Figure S7. CBP60g is weakly de-repressed in coi1 roots and not induced after infection in $\mathrm{WT}_{\text {aos }}, \mathrm{WT}_{\text {coit-t }}$ and aos.

Figure S8. SARD1/CBP60g-dependency of gene expression in the two groups of COl1suppressed but not significantly induced genes at $10 \mathrm{dpi}$ and the genes induced after infection but not under control of COI1.

Figure S9. SARD1 overexpression leads to mild growth defects in Col-0 plants.

Figure S10. WRKY54 and WRKY46 induction is stronger in shoots overexpressing SARD1 compared to roots.

Figure S11. Mutations in SARD1 and CBP60g do not affect the disease phenotype after $V$. longisporum infection. 


\section{References}

Afgan, E. et al. (2018) 'The Galaxy platform for accessible, reproducible and collaborative biomedical analyses: 2018 update', Nucleic Acids Research, 46(W1), pp. W537-W544. doi: 10.1093/nar/gky379.

Berendsen, R. L., Pieterse, C. M. J. and Bakker, P. A. H. M. (2012) 'The rhizosphere microbiome and plant health', Trends in Plant Science, 17, pp. 478-486. doi: 10.1016/j.tplants.2012.04.001.

Berendzen, K. W., Weiste, C., Wanke, D., Kilian, J., Harter, K. and Dröge-Laser, W. (2012) 'Bioinformatic cis-element analyses performed in Arabidopsis and rice disclose bZIP- and MYB-related binding sites as potential AuxRE-coupling elements in auxin-mediated transcription.', BMC plant biology, 12, p. 125. doi: 10.1186/1471-2229-12-125.

Bittel, P. and Robatzek, S. (2007) 'Microbe-associated molecular patterns (MAMPs) probe plant immunity', Current Opinion in Plant Biology, pp. 335-341. doi: 10.1016/j.pbi.2007.04.021.

Boller, T. and Felix, G. (2009) 'A renaissance of elicitors: Perception of microbe-associated molecular patterns and danger signals by pattern-recognition receptors', Annual Review of Plant Biology, 60, pp. 379-407. doi: 10.1146/annurev.arplant.57.032905.105346.

Budimir, J., Treffon, K., Nair, A., Thurow, C. and Gatz, C. (2021) 'Redox-active cysteines in TGACG-BINDING FACTOR 1 (TGA1) do not play a role in salicylic acid or pathogen-induced expression of TGA1-regulated target genes in Arabidopsis thaliana', New Phytologist, 230(6), pp. 2420-2432. doi: 10.1111/nph.16614.

Cao, H., Bowling, S. A., Gordon, A. S. and Dong, X. (1994) 'Characterization of an Arabidopsis Mutant That Is Nonresponsive to Inducers of Systemic Acquired Resistance.', The Plant Cell, 6(11), pp. 1583-1592. doi: 10.1105/TPC.6.11.1583.

Chini, A. et al. (2007) 'The JAZ family of repressors is the missing link in jasmonate signalling', Nature, 448(7154), pp. 666-671. doi: 10.1038/nature06006.

Chomczynski, P. and Mackey, K. (1995) 'Short technical reports. Modification of the TRI reagent procedure for isolation of RNA from polysaccharide- and proteoglycan-rich sources.', Biotechniques, 19(6), pp. 942-945. Available at: https://europepmc.org/article/med/8747660 (Accessed: 19 July 2021).

Clough, S. J. and Bent, A. F. (1998) 'Floral dip: A simplified method for Agrobacteriummediated transformation of Arabidopsis thaliana', Plant Journal, 16(6), pp. 735-743. doi: 10.1046/j.1365-313X.1998.00343.x.

Depotter, J. R. L., Deketelaere, S., Inderbitzin, P., Tiedemann, A. Von, Höfte, M., Subbarao, K. V., Wood, T. A. and Thomma, B. P. H. J. (2016) 'Verticillium longisporum, the invisible threat to oilseed rape and other brassicaceous plant hosts', Molecular Plant Pathology, 17(7), pp. 1004-1016. doi: 10.1111/mpp.12350.

Eynck, C., Koopmann, B., Grunewaldt-Stoecker, G., Karlovsky, P. and von Tiedemann, A. (2007) 'Differential interactions of Verticillium longisporum and V. dahliae with Brassica napus detected with molecular and histological techniques', European Journal of Plant Pathology, 118(3), pp. 259-274. doi: 10.1007/s10658-007-9144-6.

Fernández-Calvo, P. et al. (2011) 'The Arabidopsis bHLH transcription factors MYC3 and MYC4 are targets of JAZ repressors and act additively with MYC2 in the activation of jasmonate responses', Plant Cell, 23(2), pp. 701-715. doi: 10.1105/tpc.110.080788. 
Feys, B. J. F., Benedetti, C. E., Penfold, C. N. and Turner2, J. G. (1994) Arabidopsis Mutants Selected for Resistance to the Phytotoxin Coronatine Are Male Sterile, Insensitive to Methyl Jasmonate, and Resistant to a Bacterial Pathogen, The Plant Cell.

Glazebrook, J. (2005) 'Contrasting mechanisms of defense against biotrophic and necrotrophic pathogens', Annual Review of Phytopathology. Annu Rev Phytopathol, pp. 205227. doi: 10.1146/annurev.phyto.43.040204.135923.

Godard, K. A., White, R. and Bohlmann, J. (2008) 'Monoterpene-induced molecular responses in Arabidopsis thaliana', Phytochemistry, 69(9), pp. 1838-1849. doi: 10.1016/J.PHYTOCHEM.2008.02.011.

Herrera Medina, M. J., Gagnon, H., Piché, Y., Ocampo, J. A., Garcı\&\#x0301;a Garrido, J. M. and Vierheilig, H. (2003) 'Root colonization by arbuscular mycorrhizal fungi is affected by the salicylic acid content of the plant', Plant Science, 164(6), pp. 993-998. doi: 10.1016/S01689452(03)00083-9.

Hollensteiner, J. et al. (2017) 'Bacillus thuringiensis and Bacillus weihenstephanensis inhibit the growth of phytopathogenic Verticillium species', Frontiers in Microbiology, 7(JAN). doi: 10.3389/fmicb.2016.02171.

Horbach, R., Navarro-Quesada, A. R., Knogge, W. and Deising, H. B. (2011) 'When and how to kill a plant cell: Infection strategies of plant pathogenic fungi', Journal of Plant Physiology, pp. 51-62. doi: 10.1016/j.jplph.2010.06.014.

Iven, T., König, S., Singh, S., Braus-Stromeyer, S. A., Bischoff, M., Tietze, L. F., Braus, G. H., Lipka, V., Feussner, I. and Dröge-Laser, W. (2012) 'Transcriptional activation and production of tryptophan-derived secondary metabolites in arabidopsis roots contributes to the defense against the fungal vascular pathogen verticillium longisporum', Molecular Plant, 5(6), pp. 1389-1402. doi: 10.1093/mp/sss044.

Kusch, S., Thiery, S., Reinstädler, A., Gruner, K., Zienkiewicz, K., Feussner, I. and Panstruga, R. (2019) 'Arabidopsis mlo3 mutant plants exhibit spontaneous callose deposition and signs of early leaf senescence', Plant Molecular Biology, 101(1-2), pp. 21-40. doi: 10.1007/s11103-019-00877-z.

Livak, K. J. and Schmittgen, T. D. (2001) 'Analysis of relative gene expression data using real-time quantitative PCR and the 2- $\triangle \triangle C T$ method', Methods, 25(4), pp. 402-408. doi: 10.1006/meth.2001.1262.

Love, M. I., Huber, W. and Anders, S. (2014) 'Moderated estimation of fold change and dispersion for RNA-seq data with DESeq2', Genome Biology 2014 15:12, 15(12), pp. 1-21. doi: 10.1186/S13059-014-0550-8.

Martínez-Abarca, F., Herrera-Cervera, J. A., Bueno, P., Sanjuan, J., Bisseling, T. and Olivares, J. (1998) 'Involvement of Salicylic Acid in the Establishment of the Rhizobium meliloti -Alfalfa Symbiosis', Molecular Plant-Microbe Interactions $\AA$, 11(2), pp. 153-155. doi: 10.1094/MPMI.1998.11.2.153.

Martínez-Medina, A., Appels, F. V. W. and van Wees, S. C. M. (2017) 'Impact of salicylic acid- and jasmonic acid-regulated defences on root colonization by Trichoderma harzianum T78.', Plant signaling \& behavior, 12(8), p. e1345404. doi: 10.1080/15592324.2017.1345404.

Mauchline, T. H. and Malone, J. G. (2017) 'Life in earth - the root microbiome to the rescue?', Current Opinion in Microbiology, 37, pp. 23-28. doi: 10.1016/J.MIB.2017.03.005. 
McLaughlin, J. E., Bin-Umer, M. A., Widiez, T., Finn, D., McCormick, S. and Tumer, N. E. (2015) 'A lipid transfer protein increases the glutathione content and enhances Arabidopsis resistance to a trichothecene mycotoxin', PLOS ONE, 10(6). doi: 10.1371/journal.pone.0130204.

Mosblech, A., Thurow, C., Gatz, C., Feussner, I. and Heilmann, I. (2011) 'Jasmonic acid perception by COl1 involves inositol polyphosphates in Arabidopsis thaliana', Plant Journal, 65(6), pp. 949-957. doi: 10.1111/j.1365-313X.2011.04480.x.

Newman, M. A., Sundelin, T., Nielsen, J. T. and Erbs, G. (2013) 'MAMP (microbe-associated molecular pattern) triggered immunity in plants', Frontiers in Plant Science. Frontiers Research Foundation, p. 139. doi: 10.3389/fpls.2013.00139.

Ohkubo, Y., Tanaka, M., Tabata, R., Ogawa-Ohnishi, M. and Matsubayashi, Y. (2017) 'Shoot-to-root mobile polypeptides involved in systemic regulation of nitrogen acquisition', Nature Plants 2017 3:4, 3(4), pp. 1-6. doi: 10.1038/nplants.2017.29.

Okuma, N., Soyano, T., Suzaki, T. and Kawaguchi, M. (2020) 'MIR2111-5 locus and shootaccumulated mature miR2111 systemically enhance nodulation depending on HAR1 in Lotus japonicus', Nature Communications 2020 11:1, 11(1), pp. 1-13. doi: 10.1038/s41467-02019037-9.

Oome, S., Raaymakers, T. M., Cabral, A., Samwel, S., Böhm, H., Albert, I., Nürnberger, T. and Van Den Ackerveken, G. (2014) 'Nep1-like proteins from three kingdoms of life act as a microbe-associated molecular pattern in Arabidopsis', Proceedings of the National Academy of Sciences of the United States of America, 111(47), pp. 16955-16960. doi: 10.1073/pnas.1410031111.

Raaijmakers, J. M., Paulitz, T. C., Steinberg, C., Alabouvette, C. and Moënne-Loccoz, Y. (2009) 'The rhizosphere: A playground and battlefield for soilborne pathogens and beneficial microorganisms', Plant and Soil, 321(1-2), pp. 341-361. doi: 10.1007/s11104-008-9568-6.

Ralhan, A., Schöttle, S., Thurow, C., Iven, T., Feussner, I., Polle, A. and Gatz, C. (2012) 'The vascular pathogen Verticillium longisporum requires a jasmonic acid-independent COI1 function in roots to elicit disease symptoms in Arabidopsis shoots', Plant Physiology, 159(3), pp. 1192-1203. doi: 10.1104/pp.112.198598.

Reusche, M., Thole, K., Janz, D., Truskina, J., Rindfleisch, S., Drübert, C., Polle, A., Lipka, V. and Teichmanna, T. (2012) Verticillium infection triggers VASCULAR-RELATED NAC DOMAIN7-dependent de novo xylem formation and enhances drought tolerance in Arabidopsis', Plant Cell, 24(9), pp. 3823-3837. doi: 10.1105/tpc.112.103374.

Rochon, A., Boyle, P., Wignes, T., Fobert, P. R. and Després, C. (2007) 'The Coactivator Function of Arabidopsis NPR1 Requires the Core of Its BTB/POZ Domain and the Oxidation of C-Terminal Cysteines', The Plant Cell, 18(12), pp. 3670-3685. doi: 10.1105/tpc.106.046953.

Sheard, L. B. et al. (2010) 'Jasmonate perception by inositol-phosphate-potentiated COl1JAZ co-receptor', Nature, 468(7322), pp. 400-407. doi: 10.1038/nature09430.

Strawn, M. A., Marr, S. K., Inoue, K., Inada, N., Zubieta, C. and Wildermuth, M. C. (2007) 'Arabidopsis Isochorismate Synthase Functional in Pathogen-induced Salicylate Biosynthesis Exhibits Properties Consistent with a Role in Diverse Stress Responses', Journal of Biological Chemistry, 282(8), pp. 5919-5933. doi: 10.1074/JBC.M605193200.

Sun, T., Li, Y., Zhang, Q., Ding, Y., Zhang, Yuelin and Zhang, Yaxi (2015) 'ChIP-seq reveals 
broad roles of SARD1 and CBP60g in regulating plant immunity', Nature Communications, 6 . doi: 10.1038/ncomms10159.

Tabata, R., Sumida, K., Yoshii, T., Ohyama, K., Shinohara, H. and Matsubayashi, Y. (2014) 'Perception of root-derived peptides by shoot LRR-RKs mediates systemic N-demand signaling', Science, 346(6207), pp. 343-346. doi: 10.1126/SCIENCE.1257800.

Thatcher, L. F., Manners, J. M. and Kazan, K. (2009) 'Fusarium oxysporum hijacks COI1mediated jasmonate signaling to promote disease development in Arabidopsis', Plant Journal. doi: 10.1111/j.1365-313X.2009.03831.x.

Thieme, C. J. et al. (2015) 'Endogenous Arabidopsis messenger RNAs transported to distant tissues', Nature Plants, 1(4), p. 15025. doi: 10.1038/nplants.2015.25.

Thines, B., Katsir, L., Melotto, M., Niu, Y., Mandaokar, A., Liu, G., Nomura, K., He, S. Y., Howe, G. A. and Browse, J. (2007) 'JAZ repressor proteins are targets of the SCFCOI1 complex during jasmonate signalling', Nature, 448(7154), pp. 661-665. doi: 10.1038/nature05960.

Tian, T., Liu, Y., Yan, H., You, Q., Yi, X., Du, Z., Xu, W. and Su, Z. (2017) 'AgriGO v2.0: A GO analysis toolkit for the agricultural community, 2017 update', Nucleic Acids Research, 45(W1), pp. W122-W129. doi: 10.1093/nar/gkx382.

Tsikou, D., Yan, Z., Holt, D. B., Abel, N. B., Reid, D. E., Madsen, L. H., Bhasin, H., Sexauer, M., Stougaard, J. and Markmann, K. (2018) 'Systemic control of legume susceptibility to rhizobial infection by a mobile microRNA', Science, 362(6411), pp. 233-236. doi: 10.1126/SCIENCE.AAT6907.

Ulrich, L., Schmitz, J., Thurow, C. and Gatz, C. (2021) 'The jasmonoyl-isoleucine receptor CORONATINE INSENSITIVE1 suppresses defence gene expression in Arabidopsis roots independently of its ligand', The Plant Journal. doi: 10.1111/TPJ.15372.

Wang, L., Tsuda, K., Sato, M., Cohen, J. D., Katagiri, F. and Glazebrook, J. (2009) 'Arabidopsis CaM binding protein CBP60g contributes to MAMP-induced SA accumulation and is involved in disease resistance against Pseudomonas syringae', PLoS Pathogens, 5(2). doi: 10.1371/journal.ppat.1000301.

Wang, L., Tsuda, K., Truman, W., Sato, M., Nguyen, L. V., Katagiri, F. and Glazebrook, J. (2011) 'CBP60g and SARD1 play partially redundant critical roles in salicylic acid signaling', Plant Journal, 67(6), pp. 1029-1041. doi: 10.1111/j.1365-313X.2011.04655.x.

Wildermuth, M. C., Dewdney, J., Wu, G. and Ausubel, F. M. (2001) 'Erratum: Isochorismate synthase is required to synthesize salicylic acid for plant defence', Nature, 414(6863), pp. 562565. doi: $10.1038 / 35107108$.

Xie, D.-X., Feyes, B. F., James, S., Nieto-Rostro, M. and Tuner, J. (1998) 'COI1- An Arabidopsis Gene Required for Jasmonate-Regulated Defense and Fertility', Science, 280(5366), pp. 2091-2094.

Xu, L., Liu, F., Lechner, E., Genschik, P., Crosby, W. L., Ma, H., Peng, W., Huang, D. and Xie, D. (2002) 'The SCFCOI1 ubiquitin-ligase complexes are required for jasmonate response in Arabidopsis', Plant Cell, 14(8), pp. 1919-1935. doi: 10.1105/tpc.003368.

Zander, M., Thurow, C. and Gatz, C. (2014) 'TGA transcription factors activate the salicylic acid-suppressible branch of the ethylene-induced defense program by regulating ORA59 expression', Plant Physiology, 165(4), pp. 1671-1683. doi: 10.1104/pp.114.243360. 
Zeise, K. and Von Tiedemann, A. (2002) 'Host specialization among vegetative compatibility groups of Verticillium dahliae in relation to Verticillium longisporum', Journal of Phytopathology, 150(3), pp. 112-119. doi: 10.1046/j.1439-0434.2002.00730.x.

Zhang, Y., Tessaro, M. J., Lassner, M. and Li, X. (2003) 'Knockout Analysis of Arabidopsis Transcription Factors TGA2, TGA5, and TGA6 Reveals Their Redundant and Essential Roles in Systemic Acquired Resistance', The Plant Cell, 15(11), pp. 2647-2653. doi: 10.1105/tpc.014894.

Zhang, Yaxi et al. (2010a) 'Control of salicylic acid synthesis and systemic acquired resistance by two members of a plant-specific family of transcription factors.', Proceedings of the National Academy of Sciences of the United States of America, 107(42), pp. 18220-5. doi: 10.1073/pnas.1005225107. 


\section{Figure Legends}

Table 1. RNA-seq gene expression data from coi1-t, aos, $\mathrm{WT}_{\text {aos }}, \mathrm{WT}_{\text {coit-t }}$ and sid2-2 roots 10 days after mock treatment or incoculation with $1 \times 10^{6}$ spores/mL sGFP-expressing $V$. longisporum.

Table 1 will be desposited at the department of Plant Molecular Biology and Physiology, Georg-August University Göttingen, until the manuscript is published. For access, please contact Prof. C. Gatz (cgatz@gwdg.de).

Figure 1. A group of 149 genes is de-repressed in coi1-t roots and induced in aos, $\mathrm{WT}_{\text {aos }}, \mathrm{WT}_{\text {coi1-t }}$, and sid2-2 at $10 \mathrm{dpi}$.

(a) Principal component analysis of the normalised root transcriptome data acquired by RNAseq analysis 10 days after mock treatment or inoculation with $1 \times 10^{6}$ spores/mL sGFPexpressing $V$. longisporum. Biological replicates from four independent experiments are symbolised by circles (mock) or plus signs (10 dpi). For $\mathrm{WT}_{\text {coi1-t }}$ only three replicates were analysed for both mock and 10 dpi treatments. WT aos and $\mathrm{WT}_{\text {coir-t } t}$ are the wild-types obtained from the segregating offspring of heterozygous aos and coi1-t seeds. (b) Venn diagram showing the overlap between 316 genes constitutively upregulated in mock-treated coi1-t roots vs mock-treated aos, WT aos, $\mathrm{WT}_{\text {coi1-t } t}$ and sid2-2 (> 2-fold, $\left.\mathrm{p}<0.05\right)$ and 661 genes induced in aos, $\mathrm{WT}_{\text {aos }}, \mathrm{WT}_{\text {coi1-t }}$ and sid2-2 at $10 \mathrm{dpi}(>2$-fold, $\mathrm{p}<0.05)$. Expression data was obtained by RNA-seq analysis from root material 10 days after mock treatment or inoculation with $1 \times 10^{6}$ spores/mL sGFP-expressing $V$. longisporum. Circles are drawn to scale with respect to the number of genes represented in each group.

Figure 2. The 149 genes de-repressed in coil and induced after infection are related to defence responses.

Gene Ontology (GO) term enrichment analysis of the 149 genes basally upregulated in coi1-t compared to aos, $\mathrm{WT}_{\text {aos }}, \mathrm{WT}_{\text {coit-t }}$ and sid2-2 (> 2-fold; $\left.p<0.05\right)$ and induced in aos, $\mathrm{WT}_{\text {aos }}$, 
$\mathrm{WT}_{\text {coit-t }}$ and sid2-2 at $10 \mathrm{dpi}(>2$-fold, $p<0.05)$. Bars represent the fold enrichment of the number of genes found per GO term in the group of 149 genes against the number of genes found within the Arabidopsis genome associated with that GO term. Only GO terms with > 5fold enrichment against the genome are shown. SA defence related GO terms are underlined in red.

Figure 3. The binding motif for SARD1 and CBP60g 'GAAATTT' is significantly enriched in the 149 defence genes of interest.

Motif Mapper cis-element analysis of (a) the 316 genes de-repressed in coi1-t roots (coi1-t

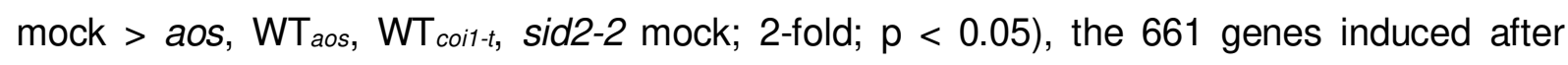

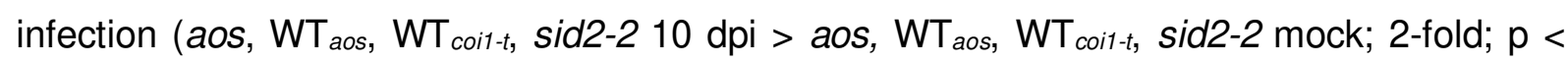
0.05), the 149 genes de-repressed in coi1-t and induced after infection, the 167 genes derepressed in coi1-t and not induced after infection and the 512 genes induced after infection but not de-repressed in coi1-t (b) the 149 genes de-repressed in coi1-t and induced after infection and the 167 genes de-repressed in coi1-t and not induced after infection against the background of the set of all 316 genes de-repressed in coi1-t roots (c) the 149 genes derepressed in coi1-t and induced after infection and the 512 genes induced after infection and not de-repressed in coi1-t roots against the background of the set of all 661 genes induced after infection. Numbers before slashes show the total number of detected motifs in the set of interest. Numbers behind the slashes show the number of expected motif counts in a set of randomly chosen promoters within the indicated background set. Significant enrichment/depletion is defined at $p<0.05$. (d) Relative expression of SARD1, DLO1, LTP4.4 and ECS1 transcript levels as quantified by RNA-seq analysis 10 days after mock treatment or inoculation with $1 \times 10^{6}$ spores/mL sGFP-expressing $V$. longisporum. Bars are means of Transcripts Per Million (TPM) \pm SEM of three to four biological replicates of each genotype, with each replicate representing twelve roots from one independent experiment. For statistical analysis, a two-way ANOVA was performed followed by Bonferroni's multiple comparison test; lowercase letters denote significant differences within each genotype between mock and 10 
dpi $(p<0.05)$, uppercase letters denote significant differences between genotypes subjected to the same treatment $(p<0.05)$. WT $T_{a s}$ and $\mathrm{WT}_{\text {coit-t }}$ are the two wild-types lines obtained from the segregating offspring of heterozygous aos and coi1-t seeds.

\section{Figure 4. Unlike in roots, COI1 is not a constitutive repressor of target genes in}

\section{shoots.}

SARD1, LTP4.4, DLO1 and ECS1 transcript levels, measured by qRT-PCR. RNA was extracted from shoots 10 days after mock treatment from the same plants whose roots were subjected to the RNA-seq. Bars are means \pm SEM of four replicates, each made up twelve shoots per genotype. For statistical analysis, a one-way ANOVA was performed followed by Tukey's multiple comparison test; lowercase letters denote significant differences between samples $(p<0.05)$. $\mathrm{WT}_{\text {aos }}$ and $\mathrm{WT}_{\text {coit-t }}$ are the two wild-types lines obtained from the segregating offspring of heterozygous aos and coi1-t seeds.

Figure 5. Genes of interest are induced independently of increased SA levels and NPR1 in roots.

(a) SARD1, LTP4.4, DLO1 and ECS1 transcript levels, measured by qRT-PCR. RNA was extracted from roots 10 days after mock treatment or infection with $1 \times 10^{6}$ spores $/ \mathrm{mL} V$. longisporum. Bars are means \pm SEM of thirteen to sixteen roots per genotype. For ECS1 transcript levels in npr1-1 only six replicates are shown. For statistical analysis, a two-way ANOVA was performed followed by Bonferroni's multiple comparison test; lowercase letters denote significant differences within each genotype between mock and 10 dpi $(p<0.05)$, uppercase letters denote significant differences between genotypes subjected to the same treatment $(p<0.05)$. (b) SARD1, LTP4.4, DLO1 and ECS1 transcript levels, measured by qRTPCR. RNA was extracted from leaves 24 hours after infiltration with $1 \mu \mathrm{M}$ nlp14. Bars are means \pm SEM of four to six replicates per genotype. For statistical analysis, a two-way ANOVA was performed followed by Bonferroni's multiple comparison test; lowercase letters denote significant differences within each genotype between mock and $1 \mu \mathrm{M}$ nlp14 $(p<0.05)$, 
uppercase letters denote significant differences between genotypes subjected to the same treatment $(p<0.05)$.

Figure 6. SARD1/CBP60g are responsible for induction of genes of interest after infection with $V$. longisporum and for basally upregulated gene expression in coi1. (a) LTP4.4, DLO1, ECS1 and ICS1 transcript levels, measured by qRT-PCR. RNA was extracted from roots 10 days after mock treatment or infection with $1 \times 10^{6}$ spores $/ \mathrm{mL} \mathrm{V}$. longisporum. Bars are means \pm SEM of thirteen to sixteen roots per genotype. For statistical analysis, a two-way ANOVA was performed followed by Bonferroni's multiple comparison test; lowercase letters denote significant differences within each genotype between mock and 10 dpi $(p<0.05)$, uppercase letters denote significant differences between genotypes subjected to the same treatment $(p<0.05)$. (b) LTP4.4, DLO1, ECS1 and ICS1 transcript levels, measured by qRT-PCR. RNA was extracted from roots 10 days after mock treatment. Bars are means \pm SEM of eight roots per genotype. For statistical analysis, a one-way ANOVA was performed followed by Tukey's multiple comparison test; lowercase letters denote significant differences between samples $(p<0.05)$.

\section{Figure 7. Overexpression of SARD1 in roots does not lead to strong target gene} activation.

SARD1, LTP4.4, DLO1, ECS1 and ICS1 transcript levels, measured by qRT-PCR. RNA was extracted from (a) roots or (b) shoots 10 days after mock treatment of SARD1 overexpression lines (SARD1 OX) and empty vector (EV) controls in both Col-0 (black bars) and sid2-2 (gray bars) background. Bars are means \pm SEM of three to six roots or shoots per line. For statistical analysis, a one-way ANOVA was performed followed by Tukey's multiple comparison test; lowercase letters denote significant differences between samples $(p<0.05)$.

Insert: Western blot of protein extracts obtained from roots and shoots of SARD1 overexpression lines (SARD1 OX) and empty vector (EV) controls in Col-0 and sid2-2 background. Per lane, six roots or three shoots were pooled from each line. C-terminally 3xHA- 
Strepll tagged SARD1 protein levels were detected using an anti-HA antibody. * depicts an unspecific band shown as loading control.

Figure 8. The SARD1/CBP60g-controlled genes upregulated in coi1 roots are not responsible for the tolerance of coi1 plants against infection with $\mathrm{V}$. Iongisporum.

Relative leaf area of plants 15 days after mock treatment or infection with $5 \times 10^{5} \mathrm{spores} / \mathrm{mL}$ sGFP-expressing $V$. longisporum. Bars are means \pm SEM of a total of $47-48$ plants per treatment from three independent experiments with 15-16 plants per treatment each. For statistical analysis, a two-way ANOVA was performed followed by Bonferroni's multiple comparison test; lowercase letters denote significant differences within each genotype between mock and 15 dpi $(p<0.05)$, uppercase letters denote significant differences between genotypes subjected to the same treatment $(p<0.05)$.

Figure 9. After V. Iongisporum infection, inactivation of COl1 leads to activation of the SARD1-branch but not the CBP60g-branch of downstream defence gene activation.

Model where $V$. longisporum infection generates a root to shoot signal (light blue) that leads to the systemic inactivation of COI1's repressive function in roots (dark blue). Inactivation of COI1 leads to activation of SARD1 and its downstream targets (green pathway) but inactivation of COI1 after $V$. longisporum infection is not sufficient to induce the CBP60g-regulated ICS1branch (red pathway). COl1 also has a direct repressive effect on SARD1-target genes like DLO1, ECS1 and LTP4.4 which is lifted after infection (green pathway). 
Table S1. Primers for Genotyping.

\begin{tabular}{|c|c|c|}
\hline & Primer ID & Sequence 5'-3' \\
\hline \multirow[t]{2}{*}{ aos } & aos-fwd & AATCGTAGGACCAATCAAAGACCG \\
\hline & aos-rev & CAGATCCTTCTTCGCTCTACCGTA \\
\hline \multirow[t]{3}{*}{ cbp60g-1 } & SALK_023199_LP & TGGTTACAGTGTCTTTAGAGCTCG \\
\hline & SALK_023199_RP & ATTCTCCTCGTTGGTCTCTACATC \\
\hline & LBb1.3 & ATTTTGCCGATTTCGGAAC \\
\hline \multirow[t]{3}{*}{ coi1-1 } & coi1-1 up & GTAATCGGAGATAGGGGTCTAGAGG \\
\hline & coi1-1 low & TGTACCCACAAGTATCTCAGTGAAGG \\
\hline & & Subsequent digestion with Mva1296I \\
\hline \multirow[t]{3}{*}{ coi1-t } & COl1gen-1936fwd & CATCTTCTGGCTTTTCTGAAACAGCTG \\
\hline & COl1gen1115rev & CACCAATTTCATTAAGGACAAAAAGTATCCAC \\
\hline & LBb1 & GCGTGGACCGCTTGCTGCAACT \\
\hline \multirow[t]{3}{*}{ sard1-1 } & SALK_138476.15.35.x_LP & GAGCATTGATCTCAGAAAACACC \\
\hline & SALK 138476.15.35.x RP & ACACTTACTTCTCCGGCAAGTAAC \\
\hline & LBb1.3 & ATTTTGCCGATTTCGGAAC \\
\hline \multirow[t]{3}{*}{ sid2-2 } & sid2-2 fwd1 & TTCTTCATGCAGGGGAGGAG \\
\hline & sid2-2 fwd2 & CAACCACCTGGTGCACCAGC \\
\hline & sid2-2 rev & AAGCAAAATGTTTGAGTCAGCA \\
\hline
\end{tabular}

Table S2. Primers for qRT-PCR.

\begin{tabular}{|l|l|}
\hline Primer ID & Sequence 5'-3' \\
\hline ANAC076 & QuantiTect QT00727076 (Qiagen) \\
\hline ERF54 & QuantiTect QT00816893 (Qiagen) \\
\hline DL01 RT fwd & AATATCGGCGACCAATGC \\
\hline DL01 RT rev & CGCTCGTTCTCGGTGTTTAC \\
\hline ECS1 & QuantiTect QT00871619 (Qiagen) \\
\hline ICS1 & QuantiTect QT00893473 (Qiagen) \\
\hline LTP4.4 & QuantiTect QT00842660 (Qiagen) \\
\hline PP2A RT fwd & AAGCAGCGTAATCGGTAGG \\
\hline PP2A RT fev & GCACAGCAATCGGGTATAAAG \\
\hline SARD1 RT fwd & TCAAGGCGTTGTGGTTTGTG \\
\hline SARD1 RT rev & CGTCAACGACGGATAGTTTC \\
\hline UBQ5 fwd RT & GACGCTTCATCTCGTCC \\
\hline UBQ5 rev RT & GTAAACGTAGGTGAGTCCA \\
\hline WRKY46 RT fwd & ACCTGCTGCTGTTGAGAATTCCG \\
\hline WRKY46 RT rev & ACGACCACAACCAATCCTGTCC \\
\hline WRKY54 & QuantiTect QT00720846 (Qiagen) \\
\hline AT5G39120 & QuantiTect QT00734748 (Qiagen) \\
\hline
\end{tabular}

Table S3. Primers for Cloning.

\begin{tabular}{|l|l|}
\hline Primer ID & Sequence 5'-3' \\
\hline SARD1GWfwd & $\begin{array}{l}\text { GGGGACAAGTTTGTACAAAAAAGCAGGCTCAACAATGGCA } \\
\text { GGGAAGAGGTTATTTCAAG }\end{array}$ \\
\hline SARD1noStopGWrev & $\begin{array}{l}\text { GGGGACCACTTTGTACAAGAAAGCTGGGTCGAAAGGGTTT } \\
\text { ATATGATTTTGAGACGAAG }\end{array}$ \\
\hline
\end{tabular}


Figure S1 (a)

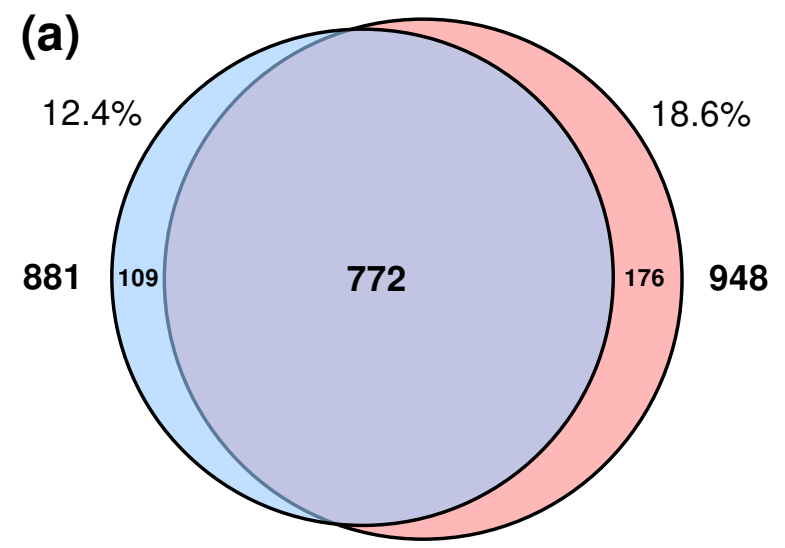

induced in $\mathrm{WT}_{\text {aos }}$ at $10 \mathrm{dpi}$

$O$ induced in $\mathrm{WT}_{\text {coit-t }}$ at $10 \mathrm{dpi}$

(b)

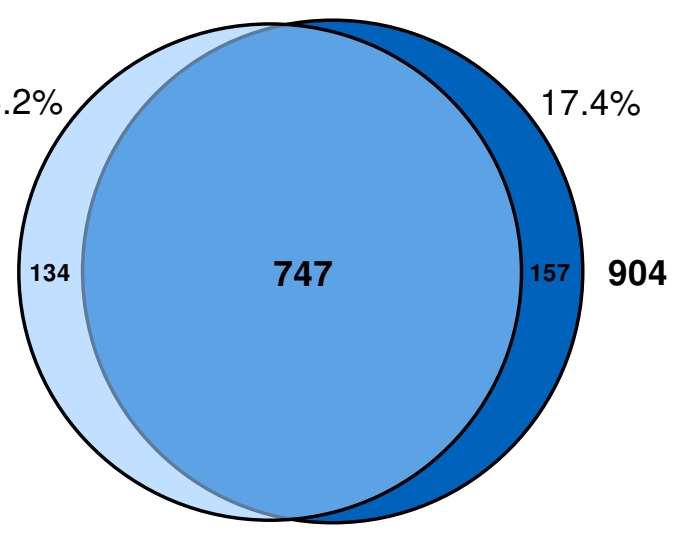

$\mathrm{O}$ induced in $\mathrm{WT}_{\text {aos }}$ at $10 \mathrm{dpi}$

induced in aos at $10 \mathrm{dpi}$

(c)

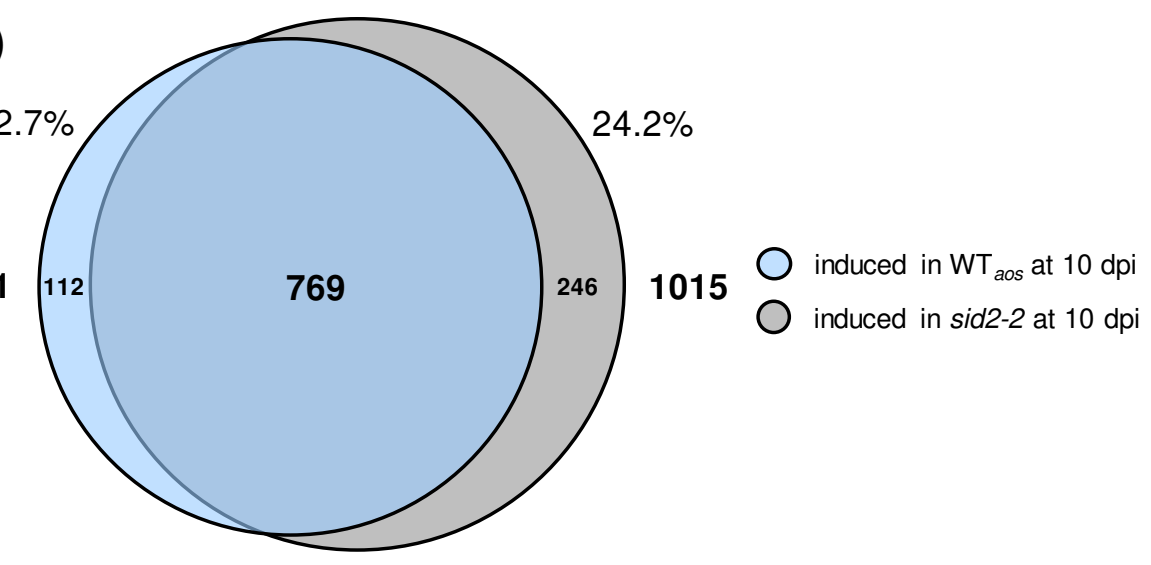

(d)

d)

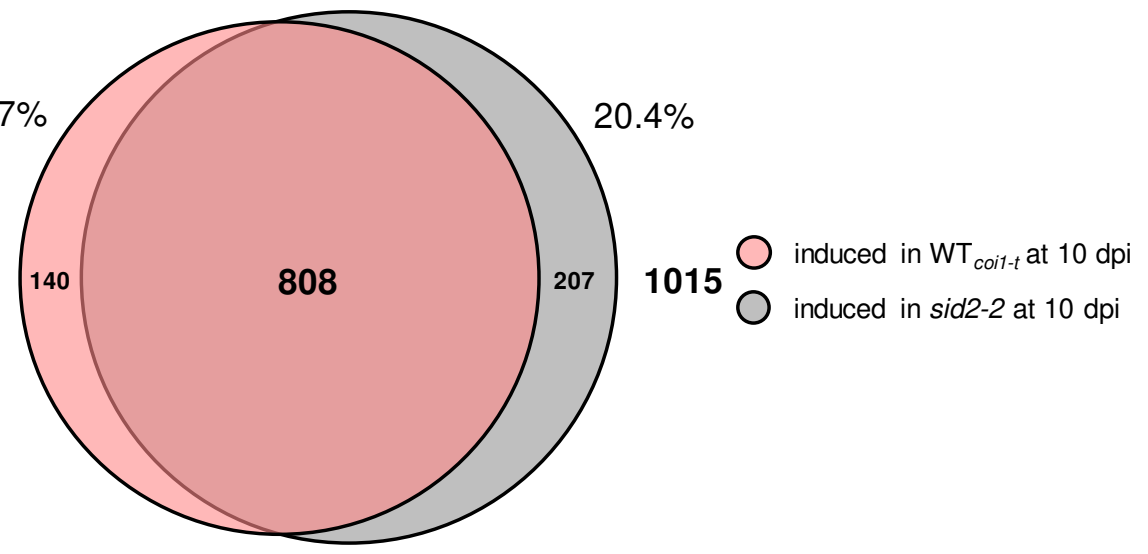

Figure S1. Gene induction patterns are largely overlapping in aos, $\mathrm{WT}_{\text {aos }}, \mathrm{WT}_{\text {coi1-t }}$, and sid2-2 at 10dpi.

Full legend on next page. 
Figure S1. Gene induction patterns are largely overlapping in aos, $\mathrm{WT}_{\text {aos }}, \mathrm{WT}_{\text {coi1-t }}$, and sid2-2 at $10 \mathrm{dpi}$.

Venn diagrams showing (a) the overlap between genes induced in $\mathrm{WT}_{\text {aos }}$ and $\mathrm{WT}_{\text {coi1-t }}$ at $10 \mathrm{dpi}(>2$ fold, $p<0.05$ ), (b) the overlap between genes induced in $\mathrm{WT}_{\text {aos }}$ and aos at $10 \mathrm{dpi}$ ( $>2$-fold, $p<0.05$ ), (c) the overlap between genes induced in $\mathrm{WT}_{\text {aos }}$ and sid2-2 at $10 \mathrm{dpi}(>2$-fold, $p<0.05$ ), (d) the overlap between genes induced in $\mathrm{WT}_{\text {coi1-t }}$ and sid2-2 at $10 \mathrm{dpi}(>2$-fold, $p<0.05)$. Expression data was obtained by RNA-seq analysis from root material 10 days after mock treatment or inoculation with $1 \times 10^{6}$ spores/mL sGFP-expressing $V$. longisporum. Circles are drawn to scale with respect to the number of genes represented in each group. $\mathrm{WT}_{\text {aos }}$ and $\mathrm{W}_{\text {coit-t }}$ are the wild-types obtained from the segregating offspring of heterozygous aos and coi1-tseeds. 
Figure S2

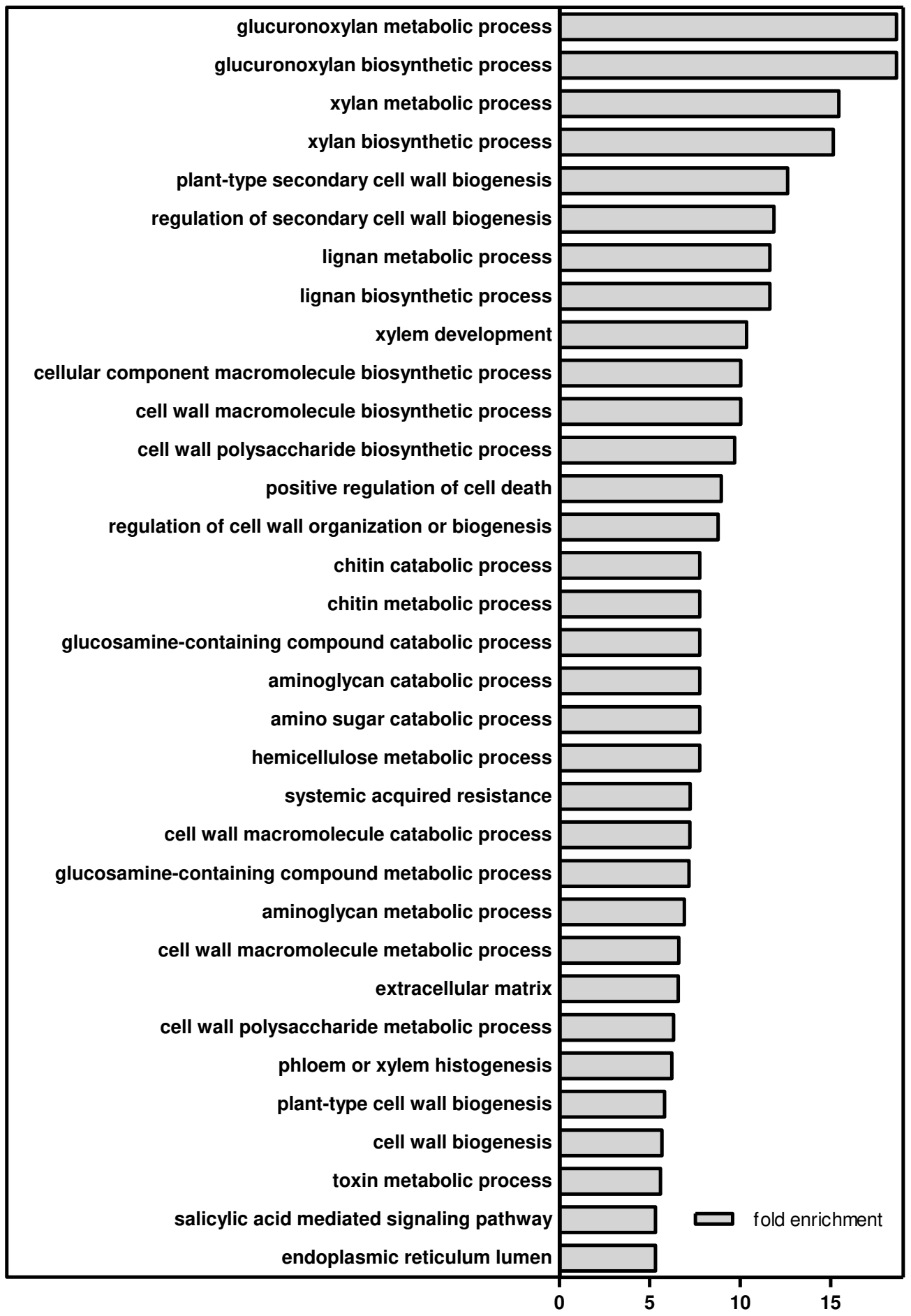

Figure S2. Gene Ontology (GO) term enrichment analysis of the $\mathbf{7 7 2}$ genes significantly induced in $\mathrm{WT}_{\text {aos }}$ and $\mathrm{WT}_{\text {coir-t }}$ at $10 \mathrm{dpi}$ (> 2-fold; $p<0.05$ ).

Bars represent fold enrichment of number of genes found per GO term in the group of 772 genes against the number of genes found within the Arabidopsis genome associated with that GO term. Only GO terms with > 5-fold enrichment against the genome are shown. 
Figure S3

(b)
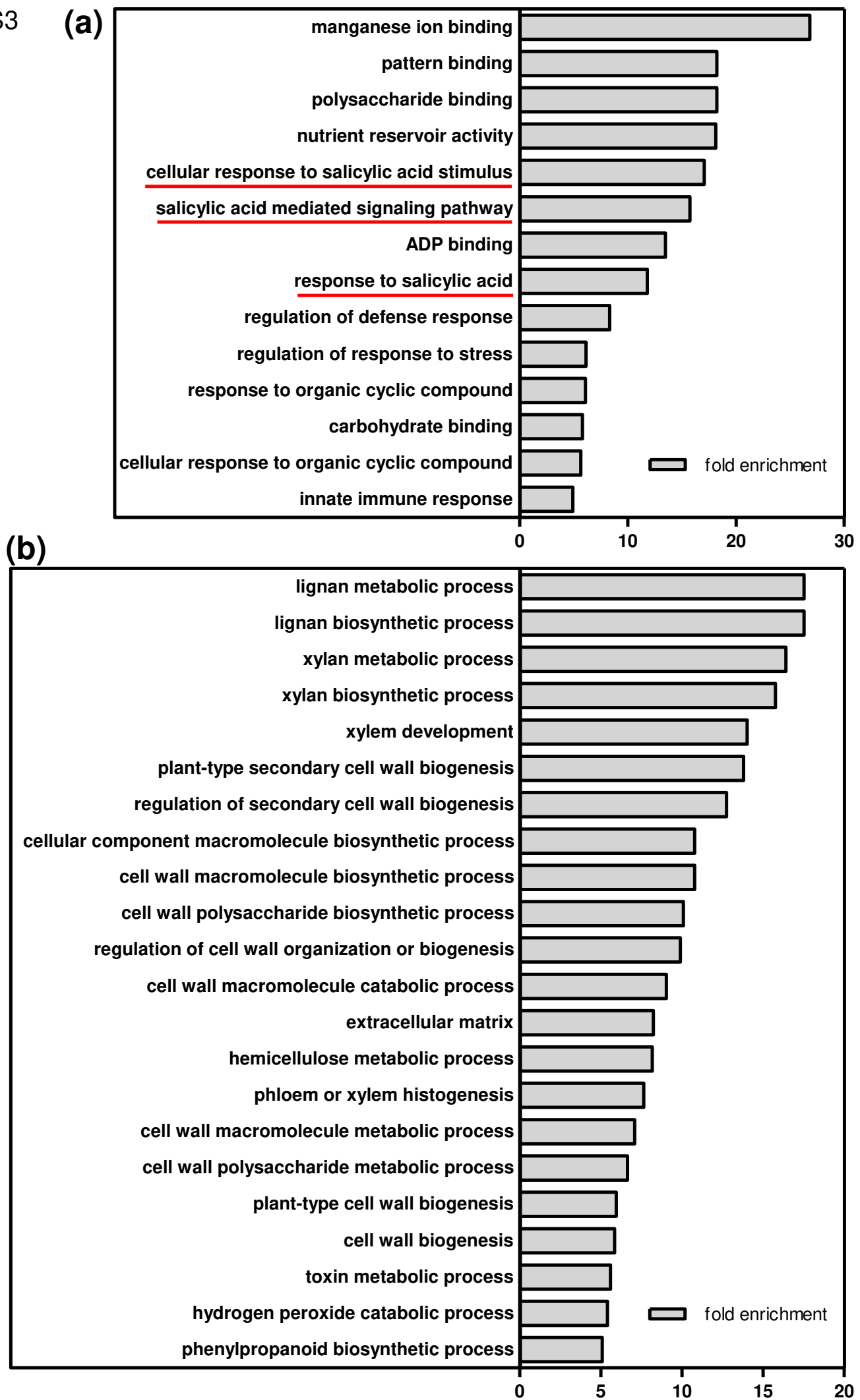

Figure S3. Gene Ontology (GO) term enrichment analysis of (a) the 167 genes basally upregulated in coi1-t compared to aos, $\mathrm{WT}_{\text {aos }}, \mathrm{WT}_{\text {coi1-t } t}$ and sid2-2 (> 2-fold; $p<0.05$ ) and not significantly induced in in aos, $\mathrm{WT}_{a o s}, \mathrm{WT}_{\text {coi1-t }}$ and sid2-2 at $10 \mathrm{dpi}$ and (b) the 512 genes induced in aos, $\mathrm{WT}_{\text {aos }}, \mathrm{WT}_{\text {coi1-t }}$ and sid2-2 at $10 \mathrm{dpi}(>2$-fold; $p<0.05$ ) but not basally upregulated in coi1-t compared to aos, $\mathrm{WT}_{\text {aos }}, \mathrm{WT}_{\text {coi1-t }}$ and sid2-2 ( $>2$-fold; $p<0.05$ ).

Full legend on next page. 
Figure S3. Gene Ontology (GO) term enrichment analysis of (a) the 167 genes upregulated in mock-treated coi1-t compared to mock-treated aos, $\mathrm{WT}_{\text {aos }}, \mathrm{WT}_{\text {coi1-t }}$ and sid2-2 (> 2-fold; $p<$ 0.05) and not significantly induced in aos, $\mathrm{WT}_{\text {aos, }} \mathrm{WT}_{\text {coi1-t }}$ and sid2-2 at $10 \mathrm{dpi}$ and (b) the 512 genes induced in aos, $\mathrm{WT}_{\text {aos, }}, \mathrm{WT}_{\text {coi1-t }}$ and sid2-2 at $10 \mathrm{dpi}(>2$-fold; $p<0.05)$ but not upregulated in mock-treated coi1-t compared to mock-treated aos, $\mathrm{WT}_{\text {aos, }}, \mathrm{WT}_{\text {coi1-t }}$ and sid2-2.

Bars represent fold enrichment of the number of genes found per GO term in the group of 167 or 512 genes against the number of genes found within the Arabidopsis genome associated with that GO term. Only GO terms with > 5-fold enrichment against the genome are shown. SA defence related GO term are underlined in red. 
Figure S4

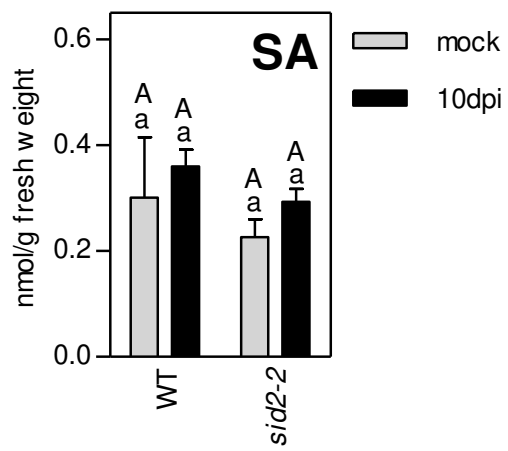

Figure S4. Infection with $\mathbf{V}$. longisporum does not lead to accumulation of SA in roots.

SA levels in roots at 10 days after mock treatment or infection with $1 \times 10^{6}$ spores $/ \mathrm{mL} V$. longisporum. Per sample eight to ten roots were pooled. Bars are means \pm SEM of two samples per genotype. For statistical analysis, a two-way ANOVA was performed followed by Bonferroni's multiple comparison test; lowercase letters denote significant differences within each genotype between mock and $10 \mathrm{dpi}$ ( $p$ $<0.05)$, uppercase letters denote significant differences between genotypes subjected to the same treatment $(p<0.05)$. 
Figure S5

(a)

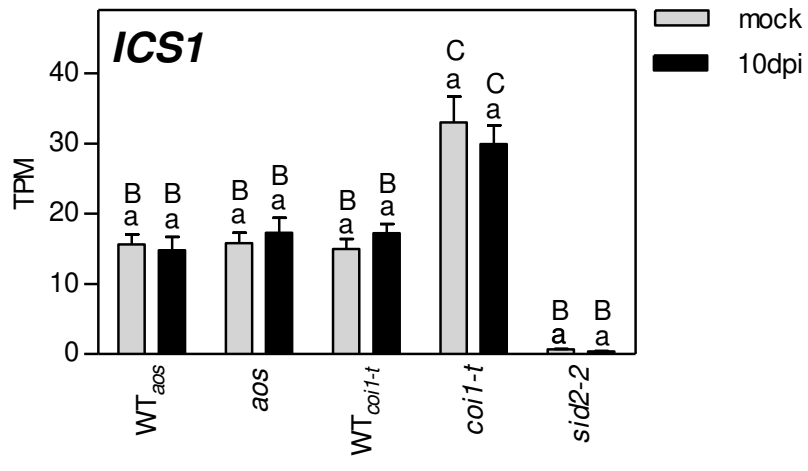

(b)

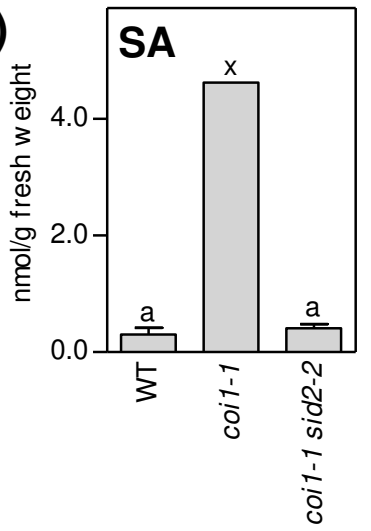

(c)

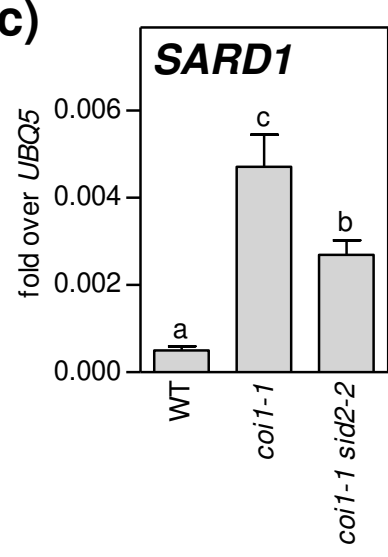

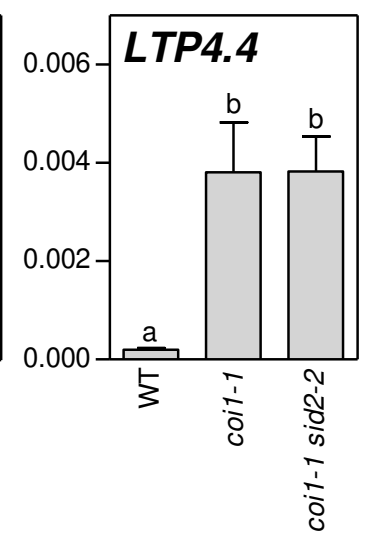

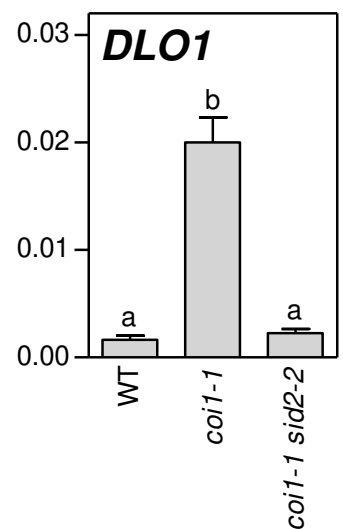

Figure S5. In coi1 roots, basally elevated marker gene expression is independent of elevated SA levels in most cases.

Full legend on next page. 
Figure S5. In coi1 roots, basally elevated marker gene expression is independent of elevated SA levels in most cases.

(a) Relative expression of ICS1 transcript levels as quantified by RNA-seq analysis 10 days after mock treatment or inoculation with $1 \times 10^{6}$ spores/mL sGFP-expressing $V$. longisporum. Bars are means of Transcripts Per Million (TPM) \pm SEM of three to four biological replicates of each genotype, with each replicate representing twelve roots from one independent experiment. For statistical analysis, a twoway ANOVA was performed followed by Bonferroni's multiple comparison test; lowercase letters denote significant differences within each genotype between mock and $10 \mathrm{dpi}(p<0.05)$, uppercase letters denote significant differences between genotypes subjected to the same treatment $(p<0.05)$. $\mathrm{WT}_{\text {aos }}$ and $\mathrm{WT}_{\text {coit- } t}$ are the two wild-types lines obtained from the segregating offspring of heterozygous aos and coi1-t seeds. (b) SA levels in roots at 10 days after mock treatment. Per sample eight to ten roots were pooled. Bars are means \pm SEM of two samples per genotype. For coi1-1 only one sample made up of eight pooled roots is shown. For statistical analysis, an unpaired Student's t-test (twotailed) was performed between WT and coi1-1 sid2-2; lowercase letters denote significant differences between samples $(p<0.05)$. $x$ denotes that coi1-1 was excluded from statistical analysis as only one replicate is shown. (c) SARD1, LTP4.4, ECS1 and DLO1 transcript levels, measured by qRT-PCR. RNA was extracted from roots 10 days after mock treatment. Bars are means \pm SEM of five to six roots per genotype. For statistical analysis, a one-way ANOVA was performed followed by Tukey's multiple comparison test; lowercase letters denote significant differences between samples $(p<0.05)$. $x$ denotes that for ECS1 levels in WT only one sample is shown as the others fell below the detection threshold in our analysis. Therefore, WT ECS1 levels had to be excluded from statistical analysis and an unpaired Student's t-test (two-tailed) was performed between WT and coi1-1 sid2-2; lowercase letters denote significant differences between samples $(p<0.05)$. 
Figure S6
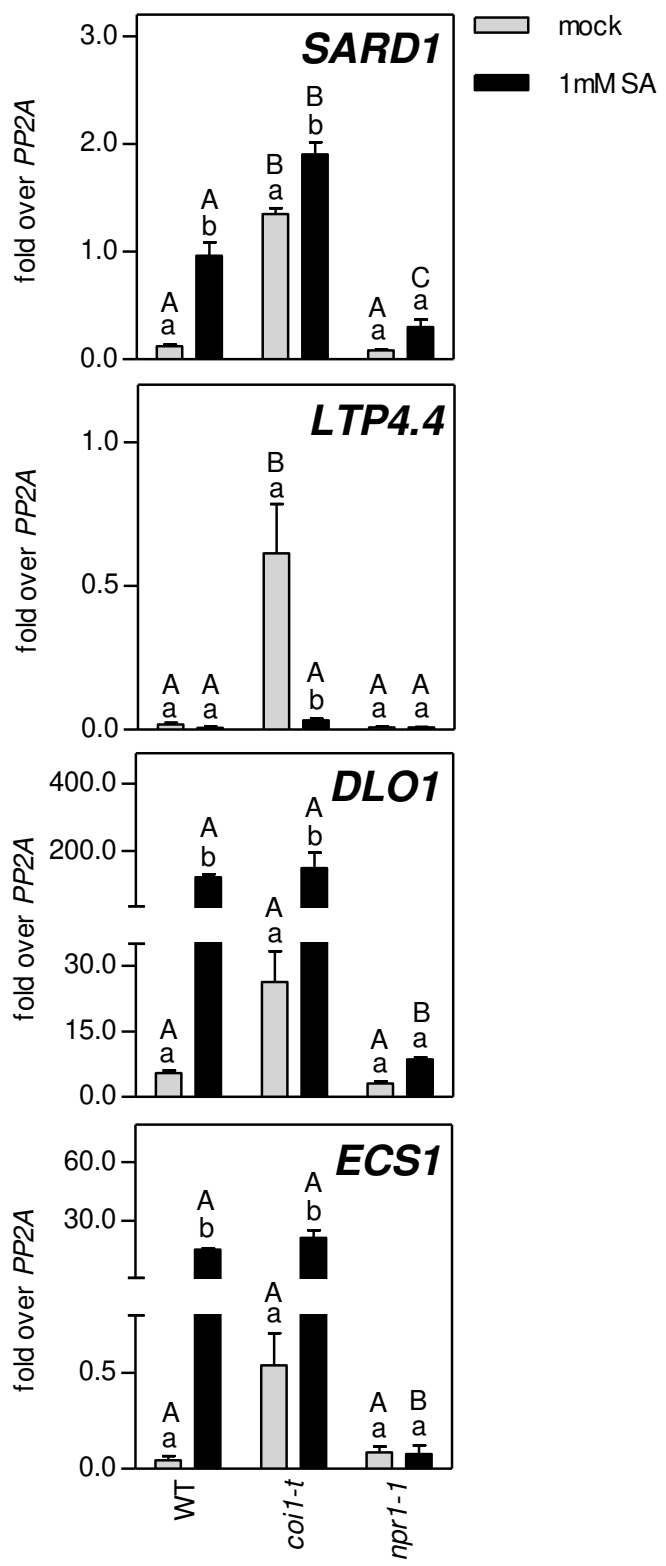

Figure S6. Genes of interest can mostly be induced by SA in roots.

SARD1, LTP4.4, DLO1 and ECS1 transcript levels, measured by qRT-PCR. RNA was extracted from roots treated with $1 \mathrm{mM} \mathrm{SA}$ for 24 hours. Per sample five to six roots were pooled. Bars are means \pm SEM of three to five replicates per genotype. For LTP4.4 transcript levels in WT roots treated with $1 \mathrm{mM}$ SA only two replicates are shown. For statistical analysis, a two-way ANOVA was performed followed by Bonferroni's multiple comparison test; lowercase letters denote significant differences within each genotype between mock and 1mM SA treatment $(p<0.05)$, uppercase letters denote significant differences between genotypes subjected to the same treatment $(p<0.05)$. 
Figure $S 7$

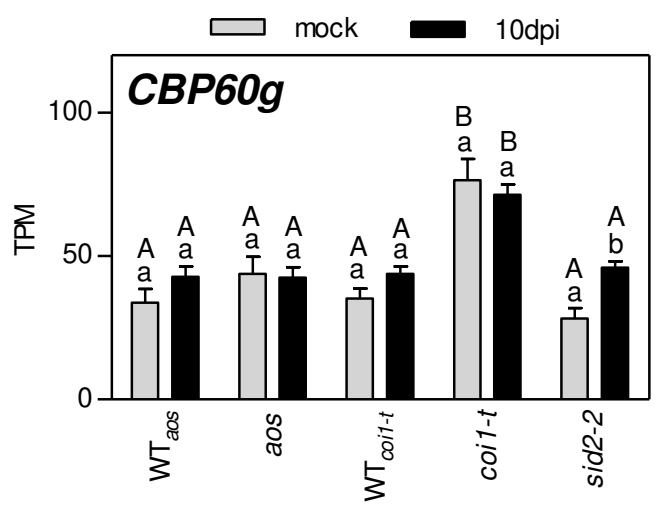

Figure S7. CBP60g is weakly de-repressed in coi1 roots and not induced after infection in $\mathrm{WT}_{\text {aos }}, \mathrm{WT}_{\text {coi1-t }}$ and aos.

Relative expression of CBP60g transcript levels as quantified by RNA-seq analysis 10 days after mock treatment or inoculation with $1 \times 10^{6}$ spores/mL sGFP-expressing $V$. longisporum. Bars are means of Transcripts Per Million (TPM) \pm SEM of three to four biological replicates of each genotype, with each replicate representing twelve roots from one independent experiment. For statistical analysis, a twoway ANOVA was performed followed by Bonferroni's multiple comparison test; lowercase letters denote significant differences within each genotype between mock and $10 \mathrm{dpi}(p<0.05)$, uppercase letters denote significant differences between genotypes subjected to the same treatment $(p<0.05)$. $\mathrm{WT}_{\text {aos }}$ and $\mathrm{WT}_{\text {coit- } t}$ are the two wild-types lines obtained from the segregating offspring of heterozygous aos and coi1-t seeds. 
Figure S8 (a)
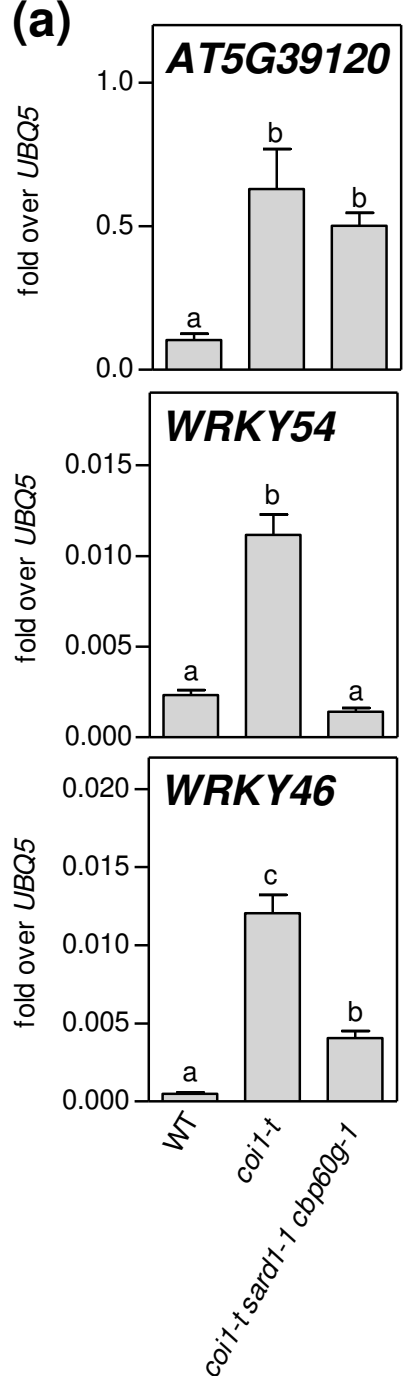

(b)

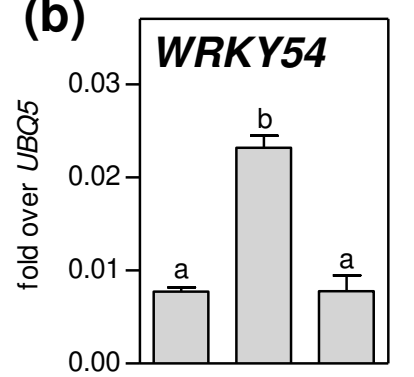

(c)
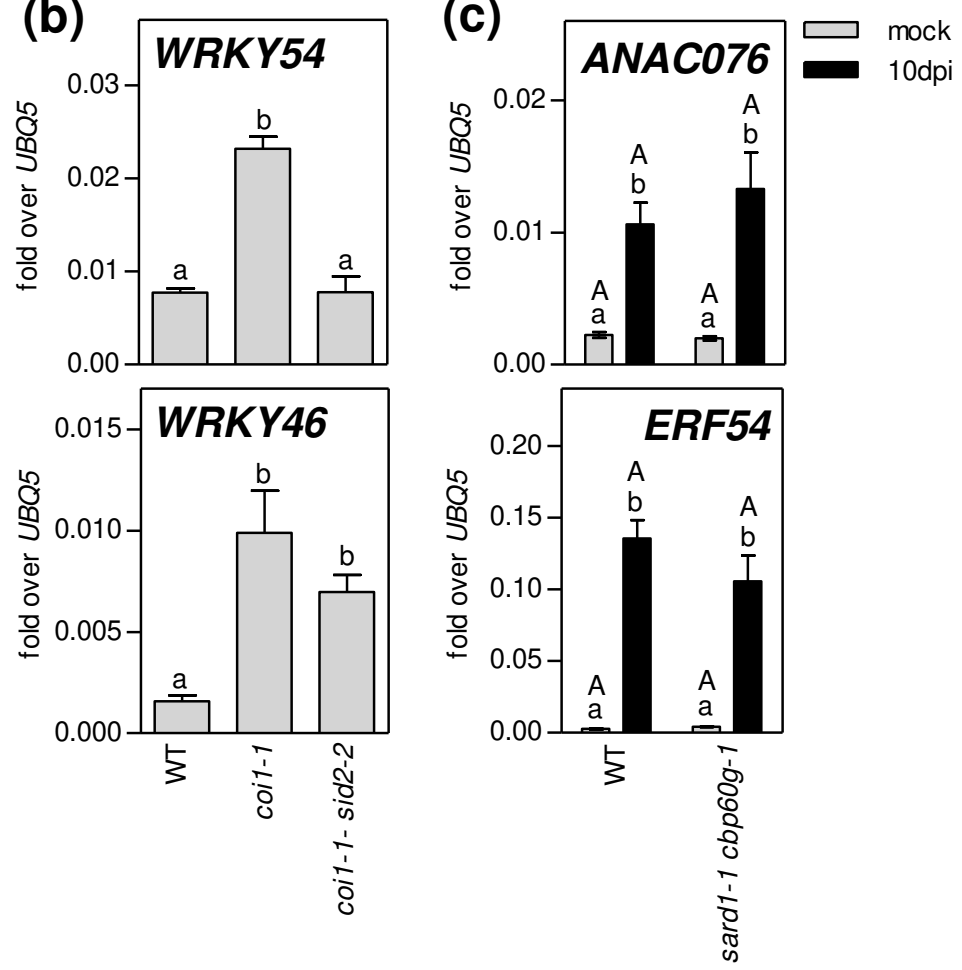

Figure S8. SARD1/CBP60g-dependency of gene expression in the two groups of COl1-

suppressed but not significantly induced genes at $10 \mathrm{dpi}$ and the genes induced after infection but not under control of COl1.

(a) AT5G39120 (a RmIC-like cupins superfamily protein), WRKY54 and WRKY46 transcript levels measured by qRT-PCR. RNA was extracted from roots 10 days after mock treatment. Bars are means \pm SEM of six to eight roots per genotype. For statistical analysis, a one-way ANOVA was performed followed by Tukey's multiple comparison test; lowercase letters denote significant differences between samples $(p<0.05)$. (b) WRKY54 and WRKY46 transcript levels measured by qRT-PCR. RNA was extracted from roots 10 days after mock treatment. Bars are means \pm SEM of six roots per genotype. For statistical analysis, a one-way ANOVA was performed followed by Tukey's multiple comparison test; lowercase letters denote significant differences between samples $(p<0.05)$. (c) ANAC076 and ERF54 transcript levels, measured by qRT-PCR. RNA was extracted from roots 10 days after mock treatment or infection with $1 \times 10^{6}$ spores $/ \mathrm{mL} V$. longisporum. Bars are means $\pm \mathrm{SEM}$ of ten to eleven roots per genotype. For statistical analysis, a two-way ANOVA was performed followed by Bonferroni's multiple comparison test; lowercase letters denote significant differences within each genotype between mock and $10 \mathrm{dpi}(p<0.05)$, uppercase letters denote significant differences between genotypes subjected to the same treatment $(p<0.05)$. 
Figure S9 (a)

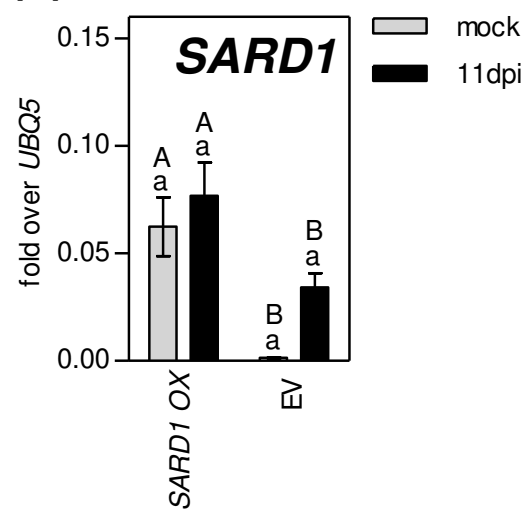

(b)

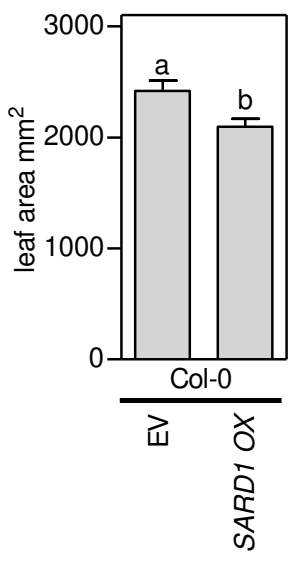

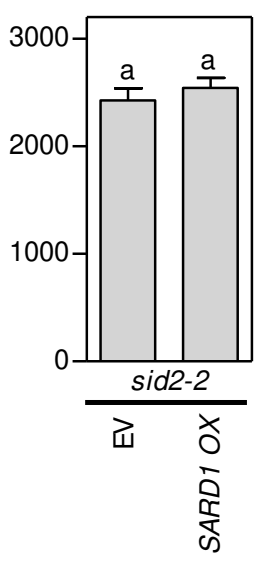

Figure S9. SARD1 overexpression leads to mild growth defects in Col-0 plants.

(a) SARD1 transcript levels, measured by qRT-PCR. RNA was extracted from roots 10 days after mock treatment or infection with $1 \times 10^{6}$ spores $/ \mathrm{mL} V$. longisporum of the $S A R D 1$ overexpression line (SARD1 OX) and empty vector (EV) control line in Col-0 background. The primers used do not differentiate between endogenous and transgenic transcript in the SARD1 OX line. Bars are means \pm SEM of five to six roots per genotype. For statistical analysis, a two-way ANOVA was performed followed by Bonferroni's multiple comparison test; lowercase letters denote significant differences within each genotype between mock and $10 \mathrm{dpi}(p<0.05)$, uppercase letters denote significant differences between genotypes subjected to the same treatment $(p<0.05)$. (b) Leaf area of SARD1 overexpression (SARD1 OX) and empty vector (EV) lines in Col-0 and sid2-2 background 15 days after mock treatment. Bars are means \pm SEM of 16 plants. For statistical analysis, an unpaired Student's ttest (two-tailed) was performed between SARD1 OX and EV; lowercase letters denote significant differences between samples $(p<0.05)$. 
Figure S10
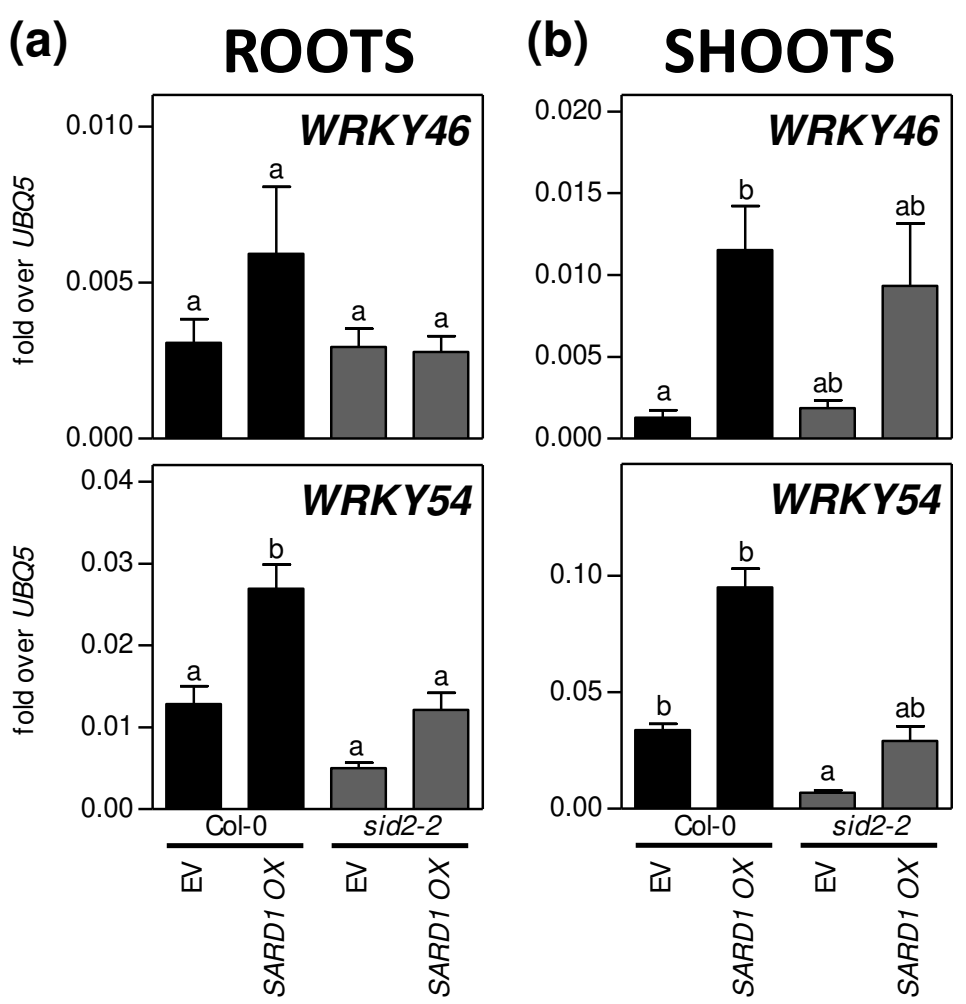

Figure S10. WRKY54 and WRKY46 induction is stronger in shoots overexpressing SARD1 compared to roots.

WRKY54 and WRKY46 transcript levels, measured by qRT-PCR. RNA was extracted from (a) roots or (b) shoots 10 days after mock treatment of SARD1 overexpression lines (SARD1 OX) and empty vector (EV) controls in both Col-0 (black bars) and sid2-2 (gray bars) background. Bars are means \pm SEM of five to six roots or shoots per line. For statistical analysis, a one-way ANOVA was performed followed by Tukey's multiple comparison test; lowercase letters denote significant differences between samples $(p<0.05)$. 
Figure S11

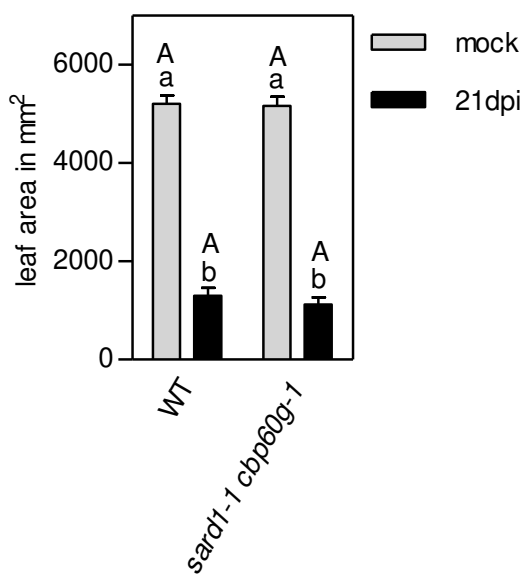

Figure S11. Mutations in SARD1 and CBP60g do not affect the disease phenotype after $V$. longisporum infection.

Leaf area of WT and sard1-1 cbp60g-1 mutants 21 days after mock treatment or infection with $1 \times 10^{6}$ spores $/ \mathrm{mL} V$. longisporum. Bars are means \pm SEM of 16 plants. For statistical analysis, a two-way ANOVA was performed followed by Bonferroni's multiple comparison test; lowercase letters denote significant differences within each genotype between mock and $21 \mathrm{dpi}(p<0.05)$, uppercase letters denote significant differences between genotypes subjected to the same treatment $(p<0.05)$. 


\section{Additional Data to Articles}

\section{Additional Data}

\subsection{The role of $A T 3 G 05770$ in facilitating susceptibility to $V$. longisporum could not be determined}

In the sand-soil root transcriptome analysis described in Article 2, we found 11 genes that are lower expressed in coi1-t after infection compared to aos, WT aos, $\mathrm{WT}_{\text {coil-t }}$ and sid2-2 roots (<2fold, $p>0.05$ ) (Article 2, Table 1 and subtables). Especially genes induced after infection in susceptible plants but not induced in the tolerant coi1-t plants would be candidates for factors that confer susceptibility to $V$. longisporum. Three genes were highly induced after infection in susceptible plants but not in coi1-t (Article 2, Table 1 and subtables). One of them is AT3G05770, a hypothetical protein (Figure AD1). Even though it showed good induction in the

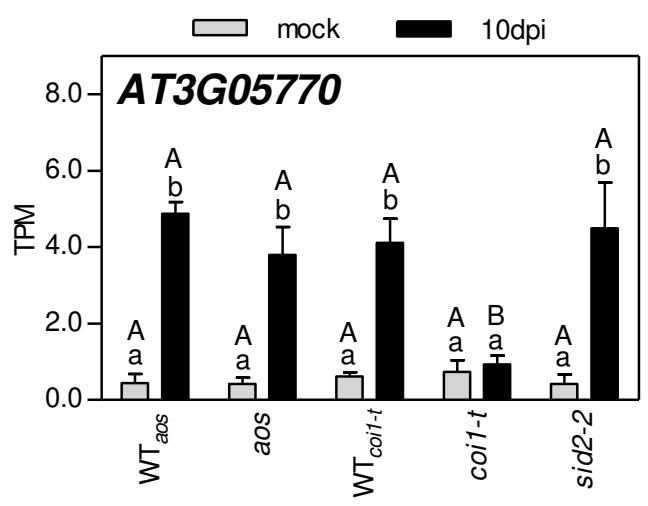

Figure AD1. AT3G05770 is induced in susceptible genotypes after infection with V. longisporum but not in the tolerant coi1-t plants.

Relative expression of AT3G05770 transcript levels as quantified by RNA-seq analysis 10 days after mock treatment or inoculation with $1 \times 10^{6}$ spores/mL sGFP-expressing $V$. longisporum (RNA-seq described in Article 2). Bars represent the average of Transcripts Per Million (TPM) \pm SEM of three to four biological replicates of each genotype, with each replicate representing twelve roots from one independent experiment. For statistical analysis, a two-way ANOVA was performed followed by Bonferroni's multiple comparison test; lowercase letters denote significant differences within each genotype between mock and $10 \mathrm{dpi}(p<0.05)$, uppercase letters denote significant differences between genotypes subjected to the same treatment $(p<0.05)$. WT $T_{\text {aos }}$ and $\mathrm{WT}_{\text {coit-t }}$ are the two wild-types lines obtained from the segregating offspring of heterozygous aos and coi1-t seeds. 
transcriptome analysis, we could not re-created induction at $10 \mathrm{dpi}$ in roots via qRT-PCR. Nevertheless, we tried to obtain knockout mutants for this gene but did not get viable homozygous offspring from heterozygous mutants we obtained from the Nottingham Arabidopsis Stock Centre (GABIseq_064G08.2, containing an insertion in the coding region). Hence, we were unable to examine whether mutants of this gene would be tolerant to infection with $V$. longisporum.

\section{$1.2 W R K Y 49$ is not a susceptibility gene enabling effective infection by $V$. longisporum}

Another gene that we found intriguing was WRKY49. It is not induced after infection, so it is not a classical susceptibility candidate gene, however, it is lower expressed in coi1-t after infection compared to the susceptible genotypes (Figure AD2a). Possibly some basal levels of WRKY49 need to be present in roots to facilitate favourable conditions for $V$. longisporum. We obtained two different wrky49 mutant lines; GABIseq_428F12.2 and SALK_091556C (both containing a T-DNA insertion in the coding region) and assessed their susceptibility to $V$. longisporum. Both wrky49 mutant lines showed similar leaf area loss compared to WT plants (Figure AD2b).

\subsection{Neither HDA6 nor SARD1 interacts with COI1 in yeast}

COI1 has been shown to interact with HISTONE DE-ACETYLASE 6 (HDA6) in a yeast two hybrid assay, in transiently transformed $A$. thaliana cell cultures but not in planta (Devoto et al., 2002). De-acetylation of histones leads to a more closed chromatin structure and reduced gene expression. We hypothesise that recruiting HDA6 to de-acetylate histones could be the mechanism by which COl1 achieves repression of genes in roots. To address this idea, we first of all aimed to reproduce the interaction between COI1 and HDA6 in the exact same yeast two hybrid system as reported by Devoto et al. (2002). Interaction experiments were performed in Saccharomyces cerevisiae using the LexA system in which COI1 was fused to the DNA 
(a)

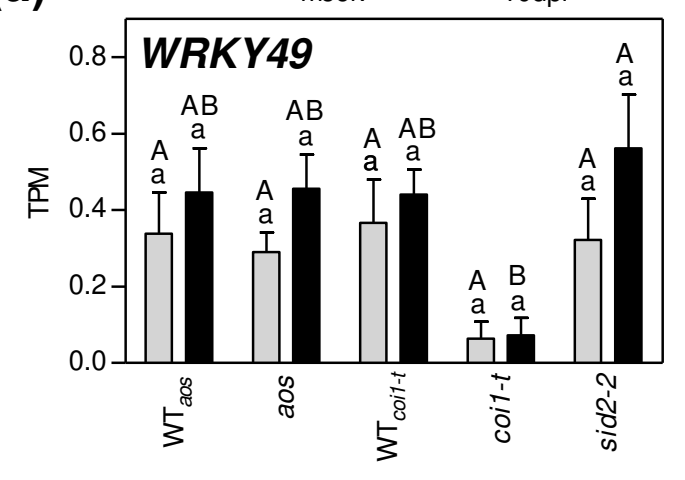

(b)

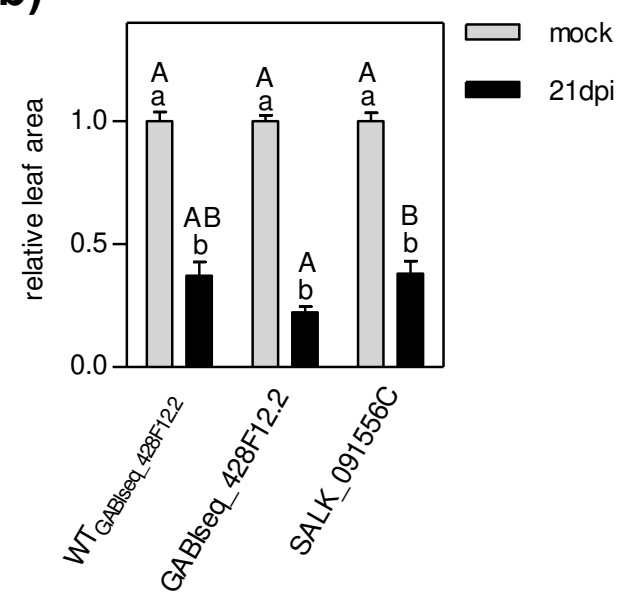

Figure AD2. WRKY49 is not a susceptibility gene facilitating effective infection by

\section{V. longisporum.}

(a) Relative expression of WRKY49 transcript levels as quantified by RNA-seq analysis $10 \mathrm{~d}$ after mock treatment or inoculation with $1 \times 10^{6}$ spores/mL sGFP-expressing $V$. longisporum. Bars represent the average of Transcripts Per Million (TPM) \pm SEM of three to four biological replicates of each genotype, with each replicate representing four roots from one independent experiment. For statistical analysis, a two-way ANOVA was performed followed by Bonferroni's multiple comparison test; lowercase letters denote significant differences within each genotype between mock and $10 \mathrm{dpi}(p<0.05)$, uppercase letters denote significant differences between genotypes subjected to the same treatment $(p<0.05)$. $W T_{\text {aos }}$ and $\mathrm{WT}_{\text {coil-t }}$ are the two wild-types lines obtained from the segregating offspring of heterozygous aos and coi1-t seeds. (b) Leaf area of WT and two wrky49 mutant lines (GABlseq_428F12.2 and SALK_091556C both containing a T-DNA insertion in the coding region) 21 days after mock treatment or infection with $1 \times 10^{6}$ spores $/ \mathrm{mL} V$. longisporum. Bars are means \pm SEM of 16 plants. For statistical analysis, a two-way ANOVA was performed followed by Bonferroni's multiple comparison test; lowercase letters denote significant differences within each genotype between mock and $10 \mathrm{dpi}(p<$ $0.05)$, uppercase letters denote significant differences between genotypes subjected to the same treatment $(p<0.05)$. $\mathrm{WT}_{\text {GABlseq_428F12.2 }}$ is the wild-type line obtained from the segregating offspring of heterozygous GABIseq_428F12.2 seeds.

binding domain (BD) and HDA6 to the activation domain (AD) (Brent and Ptashne, 1985; Van Criekinge and Beyaert, 1999). If the proteins interact, close contact between BD and AD drives expression of the LacZ gene, coding for B-galactosidase. B-Galactosidase hydrolyses the XGal added to the yeast growth medium, leading to blue staining of the yeast colonies. No interaction was observed between COI1 and HDA6 (Figure AD3a). However, as we are investigating COI1 as a repressor protein, we wondered if COI1 and HDA6 were interacting 
but were forming a repressor complex that overcame the intended activation of LacZ. To test this idea, we generated a COI1 construct that contained a DNA binding domain together with an activation domain (VP). This construct should alone be sufficient to drive activation of Lac. If COI1 and HDA6 interacted and indeed acted as a repressor together, co-transformation of COI1 in the VP construct with HDA6 should abolish activation of LacZ. However, no such repression of the active $L a c Z$ promoter was observed (Figure AD3b).

Nevertheless, as the interaction had been reported before (Devoto et al., 2002), we tested if hda6 mutant plants would show the same tolerant phenotype as coi1 plants after infection with

(a)

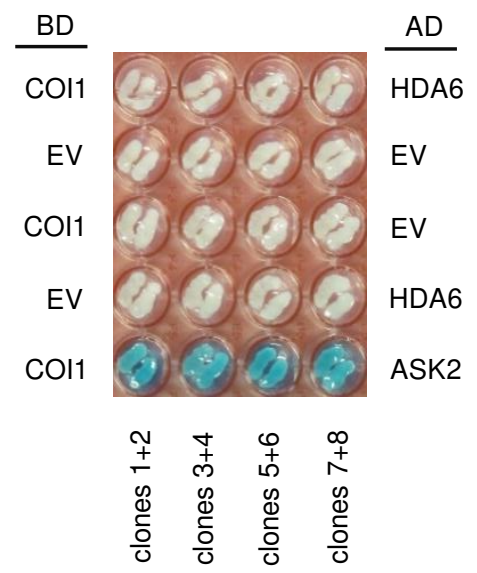

(b)

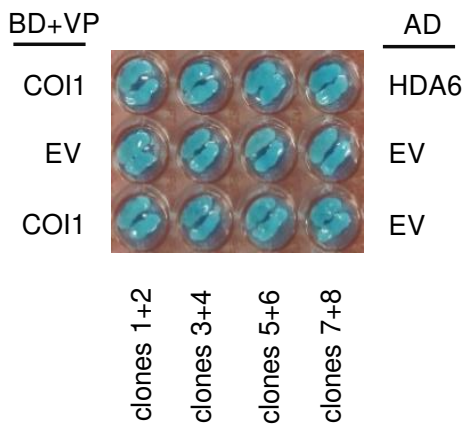

(c)

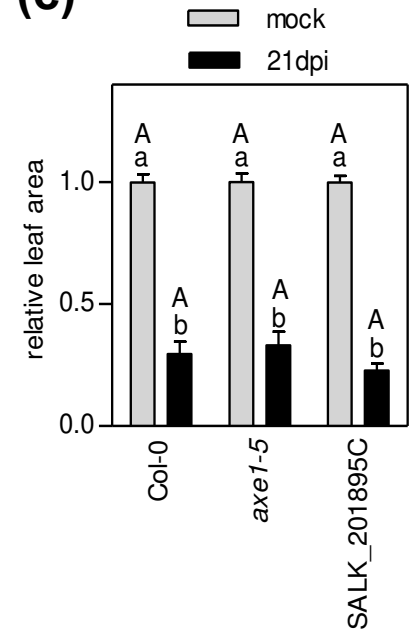

Figure AD3. HDA6 is most likely not involved in COI1-mediated repression.

(a) Interaction analysis of COI1 fused to the LexA binding domain (BD) and HDA6 fused to the B42 activation domain (AD) two days after streaking yeast colonies onto $X$-Gal supplemented medium. As a positive control the interaction between COI1 fused to the LexA binding domain and ASK2 fused to the B42 activation domain is shown. Blue colour indicates protein interaction. EV denotes empty vector controls. The experiment displayed was performed by Natalie Leutert as part of her Bachelor Thesis supervised by Louisa Ulrich. (b) COI1 fused to the LexA binding domain (BD) and the VP16 activation domain (VP) constitutes a functional unit to drive expression of $\operatorname{Lac} Z$ (indicated by blue colour) two days after streaking colonies onto X-Gal supplemented medium. HDA6 fused to the B42 activation domain (AD) was added to assess the formation of a functional repressor unit. EV denotes empty vector controls. The experiment displayed was performed by Natalie Leutert as part of her Bachelor Thesis supervised by Louisa Ulrich. (c) Leaf area of WT and two hda6 mutant lines (axe1-5 and SALK_201895C (containing a T-DNA insertion in the coding region)) 21 days after mock treatment or infection with $1 \times 10^{6}$ spores $/ \mathrm{mL} V$. longisporum. Bars are means $\pm \mathrm{SEM}$ of 16 plants. For statistical analysis, a two-way ANOVA was performed followed by Bonferroni's multiple comparison test; lowercase letters denote significant differences within each genotype between mock and $10 \mathrm{dpi}(p<$ $0.05)$, uppercase letters denote significant differences between genotypes subjected to the same treatment $(p<0.05)$. 
$V$. longisporum. If HDA6 is part of the mechanism though which COI1 achieves gene repression, then hda6 plants should show the same basally upregulated gene expression in roots. In turn, if basally upregulated gene expression in coi1 roots is the underlying reason for the tolerance, hda6 mutants should show the same tolerant pathophenotype as coi1 plants. We infected two different hda6 mutants; axe1-5 (Murfett et al., 2001) and SALK_201895C (TDNA insertion in the coding region). Both hda6 mutants were as susceptible to the fungus as WT plants (Figure AD3c).

As we show in Article 2, SARD1 is involved in the activation of genes like LTP4.4, DLO1 and ECS1. Hence, we hypothesised that SARD1 might recruit COI1 directly to promoters of genes with the GAAATTT SARD1 binding site for repression. To address this hypothesis, we again performed interaction experiments between SARD1 and COI1 in Saccharomyces cerevisiae. No interaction between COI1 and SARD1 was observed (Figure AD4).

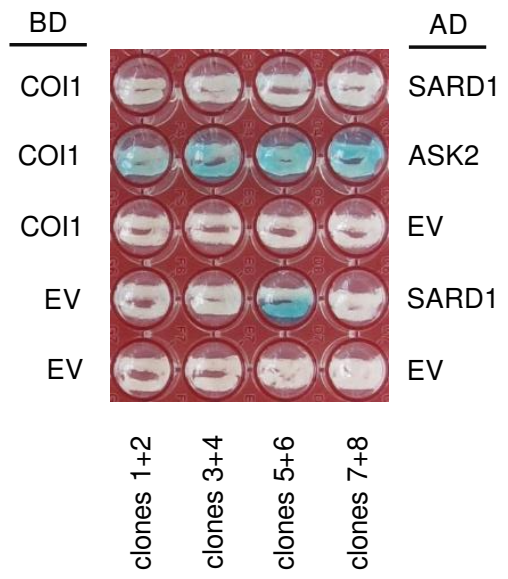

\section{Figure AD4. SARD1 is most likely not involved in COl1-mediated repression.}

Interaction analysis of COI1 fused to the LexA binding domain (BD) and SARD1 fused to the B42 activation domain $(A D)$ two days after streaking yeast colonies onto X-Gal supplemented medium. As a positive control the interaction between COI1 fused to the LexA binding domain and ASK2 fused to the B42 activation domain is shown. Blue colour indicates protein interaction. EV denotes empty vector controls. 


\section{Methods for Additional Data to Articles}

Relative expression of $A T 3 G 05770$ and $W R K Y 49$ is data from the RNA-seq analysis described in Article 2. Fungal infections and leaf area measurements were performed as described in Article 2. GABIseq_428F12.2, SALK_091556C and SALK_201895C and axe1-5 were obtained from Nottingham Arabidopsis Stock Centre (NASC). The primers for genotyping of TDNA insertion lines GABIseq_428F12.2, SALK_091556C and SALK_201895C are given in Table AD1 in Section VIII. Appendix. axe1-5 mutants were confirmed by sequencing (Murfett et al., 2001).

For interaction analyses in the LexA yeast two-hybrid system, COI1, ASK2, HDA6 and SARD1 were introduced into the GATWAY-compatible vectors pGILDA-GW and pB42AD-GW, described in Li et al. (2019). COI1 was also introduced into pGILDA-VP16-GW, additionally containing the VP16 activation domain. The sequence of pGILDA-VP16-COI1 is given in Section VIII. Appendix. Yeast two hybrid assays were performed as described in Article 1 (Ulrich et al., 2021). 


\section{General Discussion}

Prompted by the tolerance-mediating effect of coi1 roots against $V$. longisporum, RNA-seq analysis of coi1, aos and WT roots was conducted. We have observed de-repression of SArelated defence genes in coi1 but not aos roots. In Article 1 we showed that this repressor

function of COl1 operates independently from JA-lle and most likely also independently from JAZ proteins. Moreover, experiments in shoot material have shown that COI 1 only acts as a repressor of gene expression in roots. In Article 2 we describe that about $50 \%$ of the genes under negative influence of $\mathrm{COI} 1$ are induced at $10 \mathrm{dpi}$. We postulate that this part of the $\mathrm{V}$. longisporum-induced defence programme is only initiated after a fungal or plant-derived signal leads to inactivation of COI1 in roots. In addition to our findings on $\mathrm{COI} 1$ and its role in root gene expression regulation, we could show that at $10 \mathrm{dpi}$ the $V$. longisporum-induced processes in Arabidopsis roots run mostly independently of JA and SA and are in large part cell wall biogenesis-related, including xylem differentiation.

\section{Responses to $V$. longisporum infection differ at early and late time points}

GO term analysis of genes upregulated in WT plants at 10 dpi show upregulation of cell wall biogenesis proteins (Article 2, Figure S2). Especially when excluding the COl1-repressed portion of these inducible genes, which are mainly SA-defence-related genes, there are almost exclusively cell wall biosynthesis processes left (Article 2, Figure 2 and S3b). Induction of cell wall biogenesis-related genes and metabolites has previously been reported for infected $A$. thaliana shoots at $18 \mathrm{dpi}$ and $25 \mathrm{dpi}$ (Tappe, 2008; Floerl et al., 2012).

Tappe (2008) observed induction of cell wall-related genes at $18 \mathrm{dpi}$ in whole $A$. thaliana rosettes. Floerl et al. (2012) analysed the metabolome of apoplastic fluid and examined cell wall properties at $25 \mathrm{dpi}$ in $A$. thaliana leaf material. They found increased production of cell wall carbohydrates with reduced esterification and increased lignification in infected plants. 
The shared secretome of $V$. longisporum cultivated in simulated xylem medium and filtered $B$. napus xylem sap contains a large number of carbohydrate degrading enzymes (Leonard et al., 2020). This indicates that at infection stages when the fungus has entered the xylem, carbohydrate degrading enzymes are secreted. The fitness gain in producing cell wall reinforcements has been shown in a V. longisporum-resistant B. napus cultivar (Eynck et al., 2009). In comparison to a susceptible cultivar, the resistant B. napus cultivar SEM 05-500256 showed greater reinforcement of tracheary elements and build-up of vascular occlusions in the hypocotyl at 21dpi (Eynck et al., 2009). The upregulation of cell wall material production genes that we observe at $10 \mathrm{dpi}$ in roots might indicate the reinforcement of structural barriers. However, Reusche et al. (2012) observed VASCULAR-RELATED NAC DOMAIN 7 (VND7)dependent de novo xylem formation in $A$. thaliana in leaves, hypocotyl and roots, starting between 7 and 14 dpi. In our RNA-seq data from 10dpi, we found VND7 and VND6, both master regulators of xylem differentiation, in the group of non-COI1 dependent genes induced after infection (Article 2, Table 1 and subtables). Moreover, we see upregulation of MYB46 and MYB83 which coordinate biosynthesis of cellulose, hemicellulose and lignin downstream of VND6 and VND7 (Ko et al., 2014; Růžička et al., 2015). Since we do not have microscopic data from this time point, we cannot differentiate if de novoxylem formation is the sole process leading to upregulation of cell wall biogenesis genes or if the plant also reinforces barriers to restrict fungal spreading.

The induction of cell wall biogenesis programmes in $V$. longisporum infected plants at these later time points, when the fungus resides in the xylem or has reached the shoot, contrasts the responses observed at earlier time points after $V$. longisporum infection.

Studies analysing the transcriptome or translatome at 1, 2 or 3 dpi in roots show no cell wall biosynthesis-related gene expression patterns (Iven et al., 2012; Fröschel et al., 2021). Instead, they report induction of genes involved in the production of secondary metabolites, like CYP81F2 and PENETRATION 2 (PEN2) which are important for the production of indole glucosinolates (IGs) and CYP71A12, CYP71A13, PHYTOALEXIN DEFICIENT 3 (PAD3) and 
GLUTATHIONE S-TRANSFERASE 6 (GST6) which are part of the camalexin biosynthesis pathway (Iven et al., 2012; Fröschel et al., 2021).

In our transcriptome data we only see induction of CYP71A13 but not any of the other genes discussed above. Moreover, GO term analysis did not hint at any IG or camalexin biosynthesisrelated processes. In accordance, neither Tappe (2008) reports induction of these pathways at $18 \mathrm{dpi}$, nor were IGs or camalexin found to be secreted at 25dpi (Floerl et al., 2012). Hence, it seems that tryptophan-derived secondary metabolite synthesis is an early response to $V$. longisporum infection.

However, Iven et al. (2012) also saw production of camalexin and indole-3-carboxylic acid at $8 \mathrm{dpi}$. This might be an artefact of media-dependent saprophytic growth of the fungus outside the root on MS medium. Possibly, IG and camalexin production is enhanced by detection of MAMPs on the outermost root layers. The studies reporting on early transcriptome/translatome responses derived their data from infection systems on MS medium (Iven et al., 2012; Fröschel et al., 2021). In contrast, the ones describing later responses to $V$. longisporum infection, including us, used a soil-based system. In our hands, infections on MS-medium provide an environment for saprophytic growth of the fungus outside the root and did not force the fungus to enter the xylem. We observed xylem entry of $V$. longisporum in plate-based infections only when transferring plantlets to nutrient-poor agarose plates before infection. Since roots are capable of perceiving the presence of MAMPs (Millet et al., 2010; Zhou et al., 2020), extended fungal growth on the outside of roots caused by MS infection systems might lead to distorted responses. Hence, studying responses in MS plate-based systems at later timepoints, when the fungus would usually not be growing on the root surface anymore, might not accurately reflect root responses.

The combined data hints at IGs and camalexin synthesis being an early response against $V$. longisporum infection, whereas cell wall remodelling is initiated after the fungus has reached the xylem. 


\section{Regulation of COI1-mediated gene repression differs from COI1's mode of action in canonical JA signalling}

Both our RNA-seq analyses have clearly shown that COI1 is a repressor of defence gene expression in roots. As the aos mutant does not show this de-repression of gene expression, the novel COI 1 function must be JA-lle independent. To describe this repressor function of COI1, we selected marker genes identified in the RNA-seq analysis, represented by $P G M$, PRLIP2 and SARD1.

The interaction with JAZ proteins for a COI1 repressor function that requires no JA-lle would need to involve JA-lle-independent turnover of JAZs by COI1. The first idea of how COl1 might lead to gene suppression that comes to mind is similar to COI1's role in canonical JA signalling. Under basal conditions SCF ${ }^{\mathrm{CO} I 1}$ leads to ligand-independent turnover of JAZs which suppress transcription of a repressor of genes like PRLIP2, PGM and SARD1. Such a repressor would be lower expressed in coi1 roots where JAZs are not degraded. However, we could exclude the involvement of the only two candidates for such repressors found in the transcriptome. One of the potential repressor candidates did not show lower expression in coi1 compared to WT in purely soil-grown roots, where de-repression of PGM and SARD1 is still observed (Article 1, Figure S4). The other repressor candidate was so lowly expressed under these conditions that no specific PCR product could be detected.

Another possible way JAZ proteins would be involved in the repressive function of COI1 is if JAZs accumulation interfered with the action of a transcriptional repressor of genes like PGM, PRLIP2 and SARD1. Again, ligand-independent turnover of JAZs by SCFCol1 in WT and aos roots would keep JAZ proteins from over-accumulating. In contrast, in coi1 (possibly only specific) JAZs might accumulate to higher levels than even in aos and might interfere with this repressor of gene repression. However, this explanation was not supported by results from transiently transformed protoplasts, where no basal turnover of any JAZ in the absence of JAIle or coronatine was observed (Article 1, Figure S7). Nevertheless, we additionally generated a COI1 protein that is severely impaired in JAZ interaction (COI1 $\left.1_{\mathrm{AA}}\right)$. COl1 $1_{\mathrm{AA}}$ only very weakly 
complemented canonical VSP2 induction after wounding and did not restore fertility. Still, we observed highly suppressed gene expression of PGM and PRLIP2 in coi1-t/COI1 AA roots (Article 1, Figure 5).

Hence, the involvement of JAZs in the newly discovered repressor function of COI1 is unlikely. Therefore, COI1 must act differently in gene repression than it does in canonical JA signalling.

In a previous attempt, an N-terminally tagged COI1 protein could not complement gene suppression of $P G M$ and PRLIP2 in coi1 while being able to complement the canonical function (Article 1, Figure 4). The N-terminal domain of COl1 is the F-box domain, which is important for the formation of the SCF ${ }^{\mathrm{CO} 11}$ complex, which acts as an E3 ubiquitin ligase. In this functional context, an N-terminal tag does not seem to interfere with COI1 function. The F-box domain is also required for the recruitment of COI1 to JA-Ile responsive promoters by MED25, thereby establishing proximity between COI1 and JAZ. Apparently, this function is also not compromised by the $\mathrm{N}$-terminal tag. Hence, the novel repressive function of $\mathrm{COI} 1$ requires a yet unknown process that is disturbed by an N-terminal tag.

PGM and SARD1 are lower expressed in untreated roots of med25 compared to WT plants (Article 1, Figure 6). This suggests that MED25 is present at these promoters to activate basal gene expression. Since MED25 interacts with COl1 (An et al., 2017) and since it might sit at these promoters, it is a candidate for recruiting COI1.

Additionally, we investigated if COI1 might interact with HDA6 or SARD1 to carry out its repressive function. HDA6 is involved in gene repression by de-acetylation of histone $\mathrm{H} 3$ and H4 and has been shown to interact with COl1 (Devoto et al., 2002; Yu et al., 2011; Wang et al., 2013). We were unable to reproduce the interaction using exactly the same protocol (Additional data to articles, Figures AD3a and AD3b). Moreover, hda6 mutants did not show tolerance against infection with $V$. longisporum (Additional data to articles, Figure AD3c). Similarly, as we have discovered that SARD1 controls a group of COl1-repressed genes (Article 2), we hypothesised that SARD1 might recruit COl1 to these promoters. However, we also did not observe interaction of COI1 and SARD1 in yeast (Additional data to articles, Figure 
AD4). Hence, we have no evidence that suggests the involvement of either HDA6 or SARD1 in COI1-mediated gene repression.

We have shown that infection with $V$. longisporum leads to induction of COl1-suppressed genes such as LTP4.4 in a SARD1/CBP60g-dependent manner. SARD1 is itself under negative control of COI1 (Article 2, Figure 3d). Moreover, increased levels of SARD1 can only induce expression of downstream target genes in unstressed coi1 roots but not in WT roots. Therefore, COI1 must have an additional repressive effect on these downstream genes (Article 2, Figures 6 and 7). Thus, we postulate that COl1 is inactivated after infection.

Only a fraction of COl1 repressed genes is hyper-induced in coi1 after infection (Article 2, Table 1 and subtables). Hardly any genes inducible in both WTs, aos and sid2 roots are lower expressed in these genotypes compared to coi1 at $10 \mathrm{dpi}$ (Article 2, Table 1 and subtables). Hence, we hypothesise systemic inactivation of COI1 in roots after infection. The fact that the JA defence response is not induced might be evidence for the fact that COI1 is generally inactivated, not just in its role as a repressor of gene expression.

Systemic inactivation would require a systemic signal after infection. A systemic signal traveling only in the root vasculature is implausible as there is no source to sink gradient creating directional flow within the root. Instead, systemic root to shoot signals have been described where peptides, microRNAs and hormones are transported shootward with the transpirational stream and down via the source to sink gradient (Tabata et al., 2014; Ohkubo et al., 2017; Tsikou et al., 2018; Wang et al., 2019; Okuma et al., 2020). We can only speculate about the nature of such a signal so far. In our transcriptome we see induction of CLE1, coding for a small peptide hormone of the CLAVATA3 (CLV3)/EMBRYO SURROUNDING REGIONrelated (CLE) family. CLE1, 3, 4 and 7 have been shown to be induced in roots in response to low nitrogen conditions and have been proposed to travel through the phloem to systemically regulate root system architecture (Araya et al., 2014). Hence, CLE1 would be a potential candidate for inactivation of COI1 upon V. longisporum infection. Alternatively, the signal could be a volatile spreading through the root. Volatiles leading to changes in root behaviour have 
been described in the context of root-fungus interactions before (Schenkel et al., 2018; Dreher et al., 2019; Moisan et al., 2021).

If the infected plant generates the proposed signal, we assume it would be to activate the COl1-repressed root defence. On the other hand, it is possible that the mobile signal is fungusderived. Possibly, V. longisporum secretes a signal that that inactivates COI1 to avoid the launch of the JA pathway, which leads to activation of COl1-suppressed root defences unintendedly. This signal might travel up to the shoot and back down to inactivate COI1 systemically. Alternatively, a $V$. longisporum-derived molecule travels to the shoot where it is perceived by plant receptors that pass down a signal for COI1 inactivation. Tappe (2008) showed that $V$. longisporum-responsive genes are induced in shoots before fungal DNA can be detected there. Similarly, Reusche et al. (2012) report trans-differentiation in leaves in the absence of fungal hyphae. Although it is possible that the findings of Tappe (2008) are limited by PCR sensitivity, this suggests that $V$. longisporum-derived molecules can be transported to the shoot via the transpiration stream, inducing responses ahead of fungal colonisation. However, it is also possible that this signal is a DAMP generated by $V$. longisporum degrading the plant's cell walls.

The idea that COI1 repression can be overcome by pathogen infection of the root tempts to speculate that $\mathrm{COI} 1$ is a safety check for root responses. How plant roots manage and regulate perception of pathogens in an environment continuously providing exposure to MAMPs, has been a long-standing question in plant-microbe interactions. Zhou et al. (2020) have shown that differentiated roots employ a switch-like system in which the presence of MAMPs or DAMPS alone does not lead to root defence responses, instead responses are only launched when damage and perception of MAMPs coincide. Possibly, COl1 might be a similar root switch that prevents excessive triggering of root defences in response to trivial stimuli, to avoid excessive energy expenses and determent of beneficial root microbes. In line with this idea, it has been shown that elevated SA levels interfere with colonisation of roots by beneficials (Martínez-Abarca et al., 1998; Herrera Medina et al., 2003; Martínez-Medina et al., 2017). Here 
it would be interesting to see if other root pathogens can also cause inactivation of COl1 and induction of SA-related defences.

\section{Basal upregulation SARD1/CBP60g-dependent defence genes in roots is not the reason for tolerance of coi1}

As coi1 roots confer tolerance to $V$. longisporum that only later takes effect when the fungus already resides in the shoot, we hypothesised that products of de-repressed defence genes in coi1 roots travel with the transpiration stream and accumulate in the shoot. At later stages of infection when the fungus has reached the shoot its proliferation is impaired, leading to lower fungal biomass in coi1 shoots as compared to aos and WT and fewer visible infection symptoms.

By mutating SARD1 and CBP60g in the coi1 background, we could reverse pre-induction of their downstream targets (Article 2, Figure 6). However, coi1 sard1 cbp60g triple mutants were not as susceptible as WT plants (Article 2, Figure 8). With 21\% leaf area loss compared to coi1 plants with $11 \%$ loss, only a small, reproducible, albeit non statistically significant effect on leaf area loss was observed. Thus, this SARD1/CBP60g-coordinated defence programme alone is not the reason for the observed tolerance in coi1. Nevertheless, it adds a fraction to the overall tolerance observed. We initially assumed a major role of COI1 on one single type of response that would explain the tolerance. However, it is possible that a multitude of effects combined render coi1 plants tolerant against infection by $V$. longisporum.

As described earlier, cell wall reinforcements and production of IGs and camalexin are somewhat effective defences against V. longisporum infection (Eynck et al., 2009; Iven et al., 2012). We did not see basal upregulation of PEN2, CYP81F2, GST6, CYP71A12, CYP71A13 or PAD3 in coi1, so exclude that these defences are preinduced in coi1 which could explain the tolerance. 
Germin-like proteins have been shown to inhibit mycelial growth and spore germination of $V$. dahliae and F. oxysporum (Pei et al., 2019; Pei et al., 2020). Moreover, Floerl et al. (2012) detected enrichment of GLP-3 in A. thaliana apoplastic fluid after infection. Hence, GLPs present suitable candidates for root to shoot transported antifungal proteins that hamper $V$. longisporum proliferation in shoots. Indeed, our transcriptome data shows that in coi1 roots three GLPs are basally upregulated: GLP2A (AT5G39190), AT5G39160, and AT5G39120, all of which have a predicted signal peptide (Article 2, Table 1 and subtables). They are not induced in response to $V$. longisporum, however, their constitutive expression in coi1 roots and the fact that they are most likely secreted make them candidates for transport to and accumulation in the shoot. For AT5G39120 we have shown that it is still upregulated in mostly tolerant coi1 sard1 cbp60g plants, thus it remains a candidate for conferring tolerance (Article 2, Figure S8a). Hence, it would be interesting to assess if these GLPs are involved in coi1mediated tolerance to $V$. longisporum. As these GLPs are found in close proximity in the genome, a CRISPR/Cas9 approach could prove efficient for knocking out these and other GLPs simultaneously in coi1.

Besides COI1, CRT1a has been shown to facilitate infection by $V$. longisporum (Pröbsting et al., 2020). Our RNA-seq analysis yielded very few susceptibility gene candidates, that might explain the tolerance-mediating effect of coi1 roots. Only three genes which are induced in susceptible genotypes at 10dpi are not induced in coi1 after infection (Article 2, Table 1 and subtables). We aimed to address if the absence of AT3G05770 induction in coi1 (Additional data to articles, Figure AD1) might convey tolerance, however, were unable to obtain mutants for analysis. The other two candidates have not been under investigation, yet. Moreover, we tested if lower basal levels of WRKY49 might affect tolerance in response to $V$. longisporum but did not see differences in susceptibility after infection in two different wrky49 mutants (Additional data to articles, Figure AD2). 


\section{The effectiveness of SARD1-regulated defences seems to differ against $V$. longisporum and V. dahliae}

SARD1 and CBP60g have been shown to be important in defence against $V$. dahliae. $V$. dahliae possesses an effector, VdSCP41, that interacts with SARD1 and CBP60g in the nucleus (Qin et al., 2018). Arabidopsis plants infected with a mutant $V$. dahliae strain lacking VdSCF41 (VdAscp41) show increased expression levels of ICS1 and FMO1. Arabidopsis and cotton plants infected with $V d \Delta s c p 41$ show less severe disease symptoms. Qin et al. (2018) did not, however, address if sard $1 \mathrm{cbp} 60 \mathrm{~g}$ mutants were more susceptible to infection with $V$. dahliae or whether infection of sard 1 cbp60g plants with $V d \Delta s c p 41$ had an effect on susceptibility. For CBP60g at least, it has been shown that interaction with VdSCF41 reduces its DNA binding activity; probably the mechanism by which transcriptional activity on target promoters is reduced (Qin et al., 2018).

In V. longisporum-infected roots we see induction of SARD1 and activation of downstream target genes. If $V$. longisporum had an effector that interfered with SARD1 activity it is unlikely that we would see induction of SARD1-dependent targets to similar levels as in coi1. Moreover, we have shown sard1 cbp60g double mutants were not hyper-susceptible to infection compared to WT plants and mutating SARD1 and CBP60g only had a minor effect on coi1 tolerance (Article 2, Figures 8 and S11). Hence, it seems that SARD1/CBP60g do not play important roles in effective defence against $V$. longisporum.

During infection, $V$. dahliae has also been shown to secrete an effector acting as an isochorismatase (VdICS1), interfering with SA accumulation (Liu et al., 2014; Zhu et al., 2017). Indeed, we do not see an increase in SA after infection, however, we also only observe weak induction of ICS1 after infection. It is more likely that the lack of SA results from the minor induction of ICS1 than an effector hydrolysing isochorismate. 


\section{Outlook}

The findings reported here uncover a novel role of COI1 in suppression of gene expression in roots. To act as a repressor, COI1 does likely not interact with its canonical JA-signalling partners, the JAZ proteins. Pull-down experiments with the tagged COI1-lines described in Article 1 could be used to identify novel interaction partners COI1 associates with in its role as a repressor of gene expression. As pull downs might be impeded by the low endogenous concentration of COl1 in roots, generating C-terminally tagged COl1 overexpression lines might be necessary. Chromatin immunoprecipitation (ChIP)-PCR could help to elucidate if COI1 is directly present at promoters of repressed genes and also if its presence there is diminished after infection.

Based on transcriptome data we postulate that COI1 is systemically inactivated in roots of $V$. longisporum-infected plants. $\beta$-glucuronidase (GUS)-reporter lines of COI1-suppressed and highly $V$. longisporum-inducible genes like SARD1 or DLO1 in combination with a split root infection assay could be conducted to address this hypothesis. Infecting only one part of the root and analysing gene induction in distant non-infected parts of the root would help to shed light on the propagation of root responses to $V$. longisporum. Gene expression analysis of infected cle 1 roots could be conducted to address if CLE1 is involved in this potential systemic signalling pathway.

To address the idea that COl1 might act as a regulator of root defence onset, infections with other vascular and root pathogens should be conducted in A. thaliana. To see if there is any biological significance to keeping these genes suppressed, root growth assays should be considered and colonisation of coi1 roots by beneficials assessed. Investigating if COI1 also acts as a liftable repressor of root gene expression in other plant species, would enhance our understanding of the broader significance of this newly discovered COI1 function. 


\section{References}

Acosta, I. F., Gasperini, D., Chételat, A., Stolz, S., Santuari, L. and Farmer, E. E. (2013) 'Role of NINJA in root jasmonate signaling', Proceedings of the National Academy of Sciences of the United States of America, 110(38), pp. 15473-15478. doi: 10.1073/pnas.1307910110.

Aerts, N., Pereira Mendes, M. and Van Wees, S. C. M. (2021) 'Multiple levels of crosstalk in hormone networks regulating plant defense', Plant Journal, 105(2), pp. 489-504. doi: 10.1111/tpj.15124.

Albert, I., Böhm, H., Albert, M., Feiler, C. E., Imkampe, J., Wallmeroth, N., et al. (2015) 'An RLP23-SOBIR1-BAK1 complex mediates NLP-triggered immunity', Nature Plants, 1. doi: 10.1038/nplants.2015.140.

An, C., Li, L., Zhai, Q., You, Y., Deng, L., Wu, F., et al. (2017) 'Mediator subunit MED25 links the jasmonate receptor to transcriptionally active chromatin', Proceedings of the National Academy of Sciences of the United States of America, 114(42), pp. E8930-E8939. doi: 10.1073/pnas.1710885114.

Araya, T., Miyamoto, M., Wibowo, J., Suzuki, A., Kojima, S., Tsuchiya, Y. N., et al. (2014) 'CLE-CLAVATA1 peptide-receptor signaling module regulates the expansion of plant root systems in a nitrogen-dependent manner', Proceedings of the National Academy of Sciences, 111(5), pp. 2029-2034. doi: 10.1073/PNAS.1319953111.

Balint-Kurti, P. (2019) 'The plant hypersensitive response: concepts, control and consequences', Molecular Plant Pathology. Blackwell Publishing Ltd, pp. 1163-1178. doi: $10.1111 / \mathrm{mpp} .12821$.

Bari, R. and Jones, J. D. G. (2009) 'Role of plant hormones in plant defence responses', Plant Molecular Biology. Springer, pp. 473-488. doi: 10.1007/s11103-008-9435-0.

Beck, M., Wyrsch, I., Strutt, J., Wimalasekera, R., Webb, A., Boller, T., et al. (2014) 'Expression patterns of FLAGELLIN SENSING 2 map to bacterial entry sites in plant shoots and roots', Journal of Experimental Botany, 65(22), pp. 6487-6498. doi: 10.1093/JXB/ERU366.

Berlanger, I. and Powelson, M. L. (2000) 'Verticillium wilt', The Plant Health Instructor. doi: 10.1094/PHI-I-2000-0801-01.

Bernsdorff, F., Döring, A.-C., Gruner, K., Schuck, S., Bräutigam, A. and Zeier, J. (2016) 'Pipecolic Acid Orchestrates Plant Systemic Acquired Resistance and Defense Priming via Salicylic Acid-Dependent and -Independent Pathways', The Plant Cell, 28(1), pp. 102-129. doi: 10.1105/TPC.15.00496.

Bigeard, J., Colcombet, J. and Hirt, H. (2015) 'Signaling Mechanisms in Pattern-Triggered Immunity (PTI)', Molecular Plant, 8, pp. 521-539. doi: 10.1016/j.molp.2014.12.022.

Birkenbihl, R. P., Kracher, B. and Somssich, I. E. (2017) 'Induced genome-wide binding of three arabidopsis WRKY transcription factors during early MAMP-triggered immunity', Plant Cell, 29(1), pp. 20-38. doi: 10.1105/tpc.16.00681.

Brash, A. R., Baertschi, S. W., Ingram, C. D. and Harris, T. M. (1988) 'Isolation and characterization of natural allene oxides: Unstable intermediates in the metabolism of lipid hydroperoxides', Proceedings of the National Academy of Sciences of the United States of America, 85(10), pp. 3382-3386. doi: 10.1073/pnas.85.10.3382.

Breithaupt, C., Strassner, J., Breitinger, U., Huber, R., Macheroux, P., Schaller, A., et al. (2001) 'X-ray structure of 12-Oxophytodienoate reductase 1 provides structural insight into substrate binding and specificity within the family of OYE', Structure, 9(5), pp. 419-429. doi: 
10.1016/S0969-2126(01)00602-5.

Breithaupt, C., Kurzbauer, R., Lilie, H., Schaller, A., Strassner, J., Huber, R., et al. (2006) 'Crystal structure of 12-oxophytodienoate reductase 3 from tomato: Self-inhibition by dimerization', Proceedings of the National Academy of Sciences of the United States of America, 103(39), pp. 14337-14342. doi: 10.1073/pnas.0606603103.

Brent, R. and Ptashne, M. (1985) 'A eukaryotic transcriptional activator bearing the DNA specificity of a prokaryotic repressor', Cell, 43(3 PART 2), pp. 729-736. doi: 10.1016/00928674(85)90246-6.

Cao, H., Glazebrook, J. and Clarke, J. D. (1997) The Arabidopsis NPR1 Gene That Controls Systemic Acquired Resistance Encodes a Novel Protein Containing Ankyrin Repeats, Cell. Available at: http://cbil.humgen.upenn.edu/atgc/ATGCUP.html.

Chandran, D., Rickert, J., Huang, Y., Steinwand, M. A., Marr, S. K. and Wildermuth, M. C. (2014) 'Atypical E2F transcriptional repressor DEL1 acts at the intersection of plant growth and immunity by controlling the hormone salicylic acid', Cell Host and Microbe, 15(4), pp. 506-513. doi: 10.1016/j.chom.2014.03.007.

Chinchilla, D., Bauer, Z., Regenass, M., Boller, T. and Felix, G. (2006) 'The Arabidopsis receptor kinase FLS2 binds flg22 and determines the specificity of flagellin perception', Plant Cell, 18(2), pp. 465-476. doi: 10.1105/tpc.105.036574.

Chini, A., Fonseca, S., Fernández, G., Adie, B., Chico, J. M., Lorenzo, O., et al. (2007) 'The JAZ family of repressors is the missing link in jasmonate signalling', Nature, 448(7154), pp. 666-671. doi: 10.1038/nature06006.

Cho, M. H., Corea, O. R. A., Yang, H., Bedgar, D. L., Laskar, D. D., Anterola, A. M., et al. (2007) 'Phenylalanine biosynthesis in Arabidopsis thaliana: Identification and characterization of arogenate dehydratases', Journal of Biological Chemistry, 282(42), pp. 30827-30835. doi: 10.1074/jbc.M702662200.

Chung, H. S., Cooke, T. F., Depew, C. L., Patel, L. C., Ogawa, N., Kobayashi, Y., et al. (2010) 'Alternative splicing expands the repertoire of dominant JAZ repressors of jasmonate signaling', Plant Journal, 63(4), pp. 613-622. doi: 10.1111/j.1365-313X.2010.04265.x.

Cole, S. J. and Diener, A. C. (2013) 'Diversity in receptor-like kinase genes is a major determinant of quantitative resistance to Fusarium oxysporum f.sp. matthioli', New Phytologist, 200(1), pp. 172-184. doi: 10.1111/NPH.12368.

Van Criekinge, W. and Beyaert, R. (1999) 'Yeast two-hybrid: State of the art', Biological Procedures Online, 2(1), pp. 1-38. doi: 10.1251/bpo16.

Dai, L., Xu, L., Huang, D., Li, X., Luo, K. and Guan, C. (2002) 'ASK1 physically interacts with COI1 and is required for male fertility inArabidopsis', Science in China Series C: Life Sciences, 45(6), pp. 631-636. doi: 10.1007/bf02879751.

Dangl, J. L. and Jones, J. D. G. (2001) 'Plant pathogens and integrated defence responses to infection', Nature. Nature Publishing Group, pp. 826-833. doi: 10.1038/35081161.

Depotter, J. R. L., Deketelaere, S., Inderbitzin, P., Tiedemann, A. Von, Höfte, M., Subbarao, K. V., et al. (2016) 'Verticillium longisporum, the invisible threat to oilseed rape and other brassicaceous plant hosts', Molecular Plant Pathology, 17(7), pp. 1004-1016. doi: 10.1111/MPP.12350.

Devoto, A., Nieto-Rostro, M., Xie, D., Ellis, C., Harmston, R., Patrick, E., et al. (2002) 'COI1 links jasmonate signalling and fertility to the SCF ubiquitin-ligase complex in Arabidopsis', Plant Journal, 32(4), pp. 457-466. doi: 10.1046/j.1365-313X.2002.01432.x.

Dhakarey, R., Peethambaran, P. K. and Riemann, M. (2016) 'Functional Analysis of 
Jasmonates in Rice through Mutant Approaches', Plants 2016, Vol. 5, Page 15, 5(1), p. 15. doi: 10.3390/PLANTS5010015.

Diener, A. C. and Ausubel, F. M. (2005) 'RESISTANCE TO FUSARIUM OXYSPORUM 1, a Dominant Arabidopsis Disease-Resistance Gene, Is Not Race Specific', Genetics, 171(1), p. 305. doi: 10.1534/GENETICS.105.042218.

Ding, Y., Sun, T., Ao, K., Peng, Y., Zhang, Yaxi, Li, X., et al. (2018) 'Opposite Roles of Salicylic Acid Receptors NPR1 and NPR3/NPR4 in Transcriptional Regulation of Plant Immunity', Cell, 173(6), pp. 1454-1467.e15. doi: 10.1016/J.CELL.2018.03.044.

Dreher, D., Baldermann, S., Schreiner, M. and Hause, B. (2019) 'An arbuscular mycorrhizal fungus and a root pathogen induce different volatiles emitted by Medicago truncatula roots', Journal of Advanced Research, 19, pp. 85-90. doi: 10.1016/J.JARE.2019.03.002.

Dunwell, J. M., Gibbings, J. G., Mahmood, T. and Saqlan Naqvi, S. M. (2008) 'Germin and germin-like proteins: Evolution, structure, and function', Critical Reviews in Plant Sciences, 27(5), pp. 342-375. doi: 10.1080/07352680802333938.

Eberhard, J., Raesecke, H. R., Schmid, J. and Amrhein, N. (1993) 'Cloning and expression in yeast of a higher plant chorismate mutase', FEBS Letters, 334(2), pp. 233-236. doi: 10.1016/0014-5793(93)81718-F.

Emonet, A., Zhou, F., Vacheron, J., Heiman, C. M., Tendon, V. D., Ma, K.-W., et al. (2021) 'Spatially Restricted Immune Responses Are Required for Maintaining Root Meristematic Activity upon Detection of Bacteria', Current Biology, 31(5), pp. 1012-1028.e7. doi: 10.1016/J.CUB.2020.12.048.

Eynck, C., Koopmann, A. B., Grunewaldt-Stoecker, A. G., Karlovsky, A. P., Von Tiedemann, A. A., Koopmann, Á. B., et al. (2007) 'Differential interactions of Verticillium longisporum and $\mathrm{V}$. dahliae with Brassica napus detected with molecular and histological techniques', Eur J Plant Pathol, 118, pp. 259-274. doi: 10.1007/s10658-007-9144-6.

Eynck, C., Koopmann, B., Karlovsky, P. and Tiedemann, A. von (2009) 'Internal Resistance in Winter Oilseed Rape Inhibits Systemic Spread of the Vascular Pathogen Verticillium longisporum', http://dx.doi.org/10.1094/PHYTO-99-7-0802, 99(7), pp. 802-811. doi: 10.1094/PHYTO-99-7-0802.

FAO Database (2018) Food and Agriculture Organization of the United Nations, Database crops production. Available at: http://www.fao.org/faostat/en/\#data/QCL (Accessed: 24 July 2021).

Felix, G., Duran, J. D., Volko, S. and Boller, T. (1999) 'Plants have a sensitive perception system for the most conserved domain of bacterial flagellin', Plant Journal, 18(3), pp. 265276. doi: 10.1046/j.1365-313X.1999.00265.x.

Floerl, S., Majcherczyk, A., Possienke, M., Feussner, K., Tappe, H., Gatz, C., et al. (2012) 'Verticillium longisporum Infection Affects the Leaf Apoplastic Proteome, Metabolome, and Cell Wall Properties in Arabidopsis thaliana', PLOS ONE, 7(2), p. e31435. doi: 10.1371/JOURNAL.PONE.0031435.

Fonseca, S., Chini, A., Hamberg, M., Adie, B., Porzel, A., Kramell, R., et al. (2009) '(+)-7iso-Jasmonoyl-L-isoleucine is the endogenous bioactive jasmonate', Nature Chemical Biology, 5(5), pp. 344-350. doi: 10.1038/nchembio.161.

Fonseca, S., Fernández-Calvo, P., Fernández, G. M., Díez-Díaz, M., Gimenez-lbanez, S., López-Vidriero, I., et al. (2014) 'bHLH003, bHLH013 and bHLH017 are new targets of JAZ repressors negatively regulating JA responses', PLOS ONE, 9(1), p. e86182. doi: 10.1371/journal.pone.0086182.

Fradin, E. F., Zhang, Z., Ayala, J. C. J., Castroverde, C. D. M., Nazar, R. N., Robb, J., et 
al. (2009) 'Genetic Dissection of Verticillium Wilt Resistance Mediated by Tomato Ve1', Plant Physiology, 150(1), p. 320. doi: 10.1104/PP.109.136762.

Fröschel, C., Iven, T., Walper, E., Bachmann, V., Weiste, C. and Dröge-Laser, W. (2019) 'A Gain-of-Function Screen Reveals Redundant ERF Transcription Factors Providing Opportunities for Resistance Breeding Toward the Vascular Fungal Pathogen Verticillium longisporum', https://doi.org/10.1094/MPMI-02-19-0055-R, 32(9), pp. 1095-1109. doi: 10.1094/MPMI-02-19-0055-R.

Fröschel, C., Komorek, J., Attard, A., Marsell, A., Lopez-Arboleda, W. A., Berre, J. Le, et al. (2021) 'Plant roots employ cell-layer-specific programs to respond to pathogenic and beneficial microbes', Cell Host \& Microbe, 29(2), pp. 299-310.e7. doi: 10.1016/J.CHOM.2020.11.014.

Gaffney, T., Friedrich, L., Vernooij, B., Negrotto, D., Nye, G., Uknes, S., et al. (1993) 'Requirement of Salicylic Acid for the Induction of Systemic Acquired Resistance', Science, 261(5122), pp. 754-756. doi: 10.1126/SCIENCE.261.5122.754.

Gao, X., Chen, X., Lin, W., Chen, S., Lu, D., Niu, Y., et al. (2013) 'Bifurcation of Arabidopsis NLR Immune Signaling via Ca2+-Dependent Protein Kinases', PLoS Pathogens, 9(1), p. 1003127. doi: 10.1371/journal.ppat.1003127.

Gao, X., Cox, K. L. and He, P. (2014) 'Functions of calcium-dependent protein kinases in plant innate immunity', Plants. MDPI AG, pp. 160-176. doi: 10.3390/plants3010160.

Garcion, C., Lohmann, A., Lamodière, E., Catinot, J., Buchala, A., Doermann, P., et al. (2008) 'Characterization and biological function of the Isochorismate Synthase2 gene of Arabidopsis', Plant Physiology, 147(3), pp. 1279-1287. doi: 10.1104/pp.108.119420.

Glazebrook, J. (2005) 'Contrasting mechanisms of defense against biotrophic and necrotrophic pathogens', Annual Review of Phytopathology. Annu Rev Phytopathol, pp. 205227. doi: 10.1146/annurev.phyto.43.040204.135923.

Gómez-Gómez, L. and Boller, T. (2002) 'Flagellin perception: A paradigm for innate immunity', Trends in Plant Science. Trends Plant Sci, pp. 251-256. doi: 10.1016/S13601385(02)02261-6.

Gray, W. M., Hellmann, H., Dharmasiri, S. and Estelle, M. (2002) 'Role of the Arabidopsis RING-H2 protein RBX1 in RUB modification and SCF function', Plant Cell, 14(9), pp. 21372144. doi: $10.1105 /$ tpc.003178.

Hander, T., Fernández-Fernández, Á. D., Kumpf, R. P., Willems, P., Schatowitz, H., Rombaut, D., et al. (2019) 'Damage on plants activates Ca 2+-dependent metacaspases for release of immunomodulatory peptides', Science, 363(6433). doi: 10.1126/science.aar7486.

Hartmann, M., Zeier, T., Bernsdorff, F., Hö, T., Ganter, C., Rgen, J., et al. (2018) 'Flavin Monooxygenase-Generated N-Hydroxypipecolic Acid Is a Critical Element of Plant Systemic Immunity', Cell, 173, pp. 456-469. doi: 10.1016/j.cell.2018.02.049.

Hartmann, M. and Zeier, J. (2019) 'N-hydroxypipecolic acid and salicylic acid: a metabolic duo for systemic acquired resistance', Current Opinion in Plant Biology, 50, pp. 44-57. doi: 10.1016/J.PBI.2019.02.006.

Heale, J. B. and Karapapa, V. K. (1999) 'The verticillium threat to canada's major oilseed crop: Canola', Canadian Journal of Plant Pathology, 21(1), pp. 1-7. doi: 10.1080/07060661.1999.10600114.

Herrera Medina, M. J., Gagnon, H., Piché, Y., Ocampo, J. A., Garcı\&\#x0301;a Garrido, J. M. and Vierheilig, H. (2003) 'Root colonization by arbuscular mycorrhizal fungi is affected by the salicylic acid content of the plant', Plant Science, 164(6), pp. 993-998. doi: 10.1016/S01689452(03)00083-9. 
Hou, S., Liu, Z., Shen, H. and Wu, D. (2019) 'Damage-associated molecular pattern-triggered immunity in plants', Frontiers in Plant Science. Frontiers Media S.A. doi: 10.3389/fpls.2019.00646.

Howe, G. A., Major, I. T. and Koo, A. J. (2018) 'Annual Review of Plant Biology Modularity in Jasmonate Signaling for Multistress Resilience'. doi: 10.1146/annurev-arplant-042817.

Hu, Y., Jiang, L., Wang, F. and Yu, D. (2013) 'Jasmonate regulates the INDUCER OF CBF expression-C-repeat binding factor/dre binding factor1 Cascade and freezing tolerance in Arabidopsis', Plant Cell, 25(8), pp. 2907-2924. doi: 10.1105/tpc.113.112631.

Huang, H., Liu, B., Liu, L. and Song, S. (2017) 'Jasmonate action in plant growth and development', Journal of Experimental Botany, 68(6), pp. 1349-1359. doi: 10.1093/JXB/ERW495.

Huang, W., Wang, Y., Li, X. and Zhang, Y. (2020) 'Biosynthesis and Regulation of Salicylic Acid and N-Hydroxypipecolic Acid in Plant Immunity', Molecular Plant, 13(1), pp. 31-41. doi: 10.1016/J.MOLP.2019.12.008.

Huffaker, A., Pearce, G. and Ryan, C. A. (2006) 'An endogenous peptide signal in Arabidopsis activates components of the innate immune response', Proceedings of the National Academy of Sciences of the United States of America, 103(26), pp. 10098-10103. doi: 10.1073/pnas.0603727103.

Huot, B., Yao, J., Montgomery, B. L. and He, S. Y. (2014) 'Growth-Defense Tradeoffs in Plants: A Balancing Act to Optimize Fitness', Molecular Plant, 7(8), pp. 1267-1287. doi: 10.1093/MP/SSU049.

Inderbitzin, P., Bostock, R. M., Davis, R. M., Usami, T., Platt, H. W. and Subbarao, K. V. (2011) 'Phylogenetics and Taxonomy of the Fungal Vascular Wilt Pathogen Verticillium, with the Descriptions of Five New Species', PLoS ONE. Edited by A. Idnurm, 6(12), p. e28341. doi: 10.1371/journal.pone.0028341.

Inderbitzin, P., Davis, R. M., Bostock, R. M. and Subbarao, K. V. (2011) 'The Ascomycete Verticillium longisporum Is a Hybrid and a Plant Pathogen with an Expanded Host Range', PLOS ONE, 6(3), p. e18260. doi: 10.1371/JOURNAL.PONE.0018260.

Ingram, R. (1968) 'Verticillium dahliae var. longisporum, a stable diploid', Transactions of the British Mycological Society, 51(2), pp. 339-341. doi: 10.1016/S0007-1536(68)80073-7.

Innes, R. (2018) 'The Positives and Negatives of NPR: A Unifying Model for Salicylic Acid Signaling in Plants', Cell, 173(6), pp. 1314-1315. doi: 10.1016/J.CELL.2018.05.034.

Iven, T., König, S., Singh, S., Braus-Stromeyer, S. A., Bischoff, M., Tietze, L. F., et al. (2012) 'Transcriptional activation and production of tryptophan-derived secondary metabolites in arabidopsis roots contributes to the defense against the fungal vascular pathogen verticillium longisporum', Molecular Plant, 5(6), pp. 1389-1402. doi: 10.1093/mp/sss044.

Jiang, Y., Liang, G., Yang, S. and Yu, D. (2014) 'Arabidopsis WRKY57 functions as a node of convergence for jasmonic acid- and auxin-mediated signaling in jasmonic acid-induced leaf senescence', Plant Cell, 26(1), pp. 230-245. doi: 10.1105/tpc.113.117838.

Johansson, A., Staal, J. and Dixelius, C. (2006) 'Early Responses in the ArabidopsisVerticillium longisporum Pathosystem Are Dependent on NDR1, JA- and ET-Associated Signals via Cytosolic NPR1 and RFO1', Molecular Plant-Microbe Interactions, 19(9), pp. 958969. doi: 10.1094/MPMI-19-0958.

Jones, J. D. G. and Dangl, J. L. (2006) 'The plant immune system', Nature. Nature Publishing Group, pp. 323-329. doi: 10.1038/nature05286.

De Jonge, R., Esse, H. P. van, Maruthachalam, K., Bolton, M. D., Santhanam, P., Saber, 
M. K., et al. (2012) 'Tomato immune receptor Ve1 recognizes effector of multiple fungal pathogens uncovered by genome and RNA sequencing', Proceedings of the National Academy of Sciences, 109(13), pp. 5110-5115. doi: 10.1073/PNAS.1119623109.

Kadota, Y., Shirasu, K. and Zipfel, C. (2015) 'Regulation of the NADPH Oxidase RBOHD during Plant Immunity', Plant and Cell Physiology, 56(8), pp. 1472-1480. doi: 10.1093/pcp/pcv063.

Kagale, S., Links, M. G. and Rozwadowski, K. (2010) 'Genome-wide analysis of ethyleneresponsive element binding factor-associated amphiphilic repression motif-containing transcriptional regulators in arabidopsis', Plant Physiology, 152(3), pp. 1109-1134. doi: 10.1104/pp.109.151704.

Karapapa, V. K., Bainbridge, B. W. and Heale, J. B. (1997) 'Morphological and molecular characterization of Verticillium longisporum comb, nov., pathogenic to oilseed rape', Mycological Research, 101(11), pp. 1281-1294. doi: 10.1017/S0953756297003985.

Kawchuk, L. M., Hachey, J., Lynch, D. R., Kulcsar, F., Rooijen, G. van, Waterer, D. R., et al. (2001) 'Tomato Ve disease resistance genes encode cell surface-like receptors', Proceedings of the National Academy of Sciences, 98(11), pp. 6511-6515. doi: 10.1073/PNAS.091114198.

Keinath, N. F., Waadt, R., Brugman, R., Schroeder, J. I., Grossmann, G., Schumacher, K., et al. (2015) 'Live Cell Imaging with R-GECO1 Sheds Light on flg22- and Chitin-Induced Transient [Ca2+]cyt Patterns in Arabidopsis', Molecular Plant, 8(8), pp. 1188-1200. doi: 10.1016/J.MOLP.2015.05.006.

Kim, C. Y. and Zhang, S. (2004) 'Activation of a mitogen-activated protein kinase cascade induces WRKY family of transcription factors and defense genes in tobacco', Plant Journal, 38(1), pp. 142-151. doi: 10.1111/j.1365-313X.2004.02033.x.

Kim, Y., Park, S., Gilmour, S. J. and Thomashow, M. F. (2013) 'Roles of CAMTA transcription factors and salicylic acid in configuring the low-temperature transcriptome and freezing tolerance of Arabidopsis', The Plant Journal, 75(3), pp. 364-376. doi: 10.1111/TPJ.12205.

Kim, Y., Gilmour, S. J., Chao, L., Park, S. and Thomashow, M. F. (2020) 'Arabidopsis CAMTA Transcription Factors Regulate Pipecolic Acid Biosynthesis and Priming of Immunity Genes', Molecular Plant, 13(1), pp. 157-168. doi: 10.1016/J.MOLP.2019.11.001.

Kloek, A. P., Verbsky, M. L., Sharma, S. B., Schoelz, J. E., Vogel, J., Klessig, D. F., et al. (2001) 'Resistance to Pseudomonas syringae conferred by an Arabidopsis thaliana coronatine-insensitive (coi1) mutation occurs through two distinct mechanisms', The Plant Journal, 26(5), pp. 509-522. doi: 10.1046/J.1365-313X.2001.01050.X.

Knecht, K., Seyffarth, M., Desel, C., Thurau, T., Sherameti, I., Lou, B., et al. (2010) 'Expression of BvGLP-1 Encoding a Germin-Like Protein from Sugar Beet in Arabidopsis thaliana Leads to Resistance Against Phytopathogenic Fungi', http://dx.doi.org/10.1094/MPMI-23-4-0446, 23(4), pp. 446-457. doi: 10.1094/MPMI-23-40446.

Ko, D. and Helariutta, Y. (2017) 'Shoot-Root Communication in Flowering Plants', Current Biology, 27(17), pp. R973-R978. doi: 10.1016/J.CUB.2017.06.054.

Ko, J.-H., Jeon, H.-W., Kim, W.-C., Kim, J.-Y. and Han, K.-H. (2014) 'The MYB46/MYB83mediated transcriptional regulatory programme is a gatekeeper of secondary wall biosynthesis', Annals of Botany, 114(6), p. 1099. doi: 10.1093/AOB/MCU126.

Koo, A. J. K., Cooke, T. F. and Howe, G. A. (2011) 'Cytochrome P450 CYP94B3 mediates catabolism and inactivation of the plant hormone jasmonoyl-L-isoleucine', Proceedings of the National Academy of Sciences of the United States of America, 108(22), pp. 9298-9303. doi: 
10.1073/pnas.1103542108.

Kubicek, C. P., Starr, T. L. and Glass, N. L. (2014) 'Plant cell wall-degrading enzymes and their secretion in plant-pathogenic fungi', Annual Review of Phytopathology, 52, pp. 427-451. doi: 10.1146/annurev-phyto-102313-045831.

Lee, M., Jeon, H. S., Kim, S. H., Chung, J. H., Roppolo, D., Lee, H., et al. (2019) 'Ligninbased barrier restricts pathogens to the infection site and confers resistance in plants', The EMBO Journal, 38(23), p. e101948. doi: 10.15252/embj.2019101948.

Lefevere, H., Bauters, L. and Gheysen, G. (2020) 'Salicylic Acid Biosynthesis in Plants', Frontiers in Plant Science. Frontiers Media S.A., p. 338. doi: 10.3389/fpls.2020.00338.

Leon-Reyes, A., Does, D. Van der, Lange, E. S. De, Delker, C., Wasternack, C., Wees, S. C. M. Van, et al. (2010) 'Salicylate-mediated suppression of jasmonate-responsive gene expression in Arabidopsis is targeted downstream of the jasmonate biosynthesis pathway', Planta 2010 232:6, 232(6), pp. 1423-1432. doi: 10.1007/S00425-010-1265-Z.

Leonard, M., Kühn, A., Harting, R., Maurus, I., Nagel, A., Starke, J., et al. (2020) 'Verticillium longisporum Elicits Media-Dependent Secretome Responses With Capacity to Distinguish Between Plant-Related Environments', Frontiers in Microbiology, 11. doi: 10.3389/FMICB.2020.01876.

Li, J., Brader, G., Kariola, T. and Palva, E. T. (2006) 'WRKY70 modulates the selection of signaling pathways in plant defense', The Plant Journal, 46(3), pp. 477-491. doi: 10.1111/J.1365-313X.2006.02712.X.

Li, J., Brader, G. and Palva, E. T. (2004) 'The WRKY70 transcription factor: a node of convergence for jasmonate-mediated and salicylate-mediated signals in plant defense.', The Plant cell, 16(2), pp. 319-31. doi: 10.1105/tpc.016980.

Li, N., Muthreich, M., Huang, L.-J., Thurow, C., Sun, T., Zhang, Y., et al. (2019) 'TGACGBINDING FACTORs (TGAs) and TGA-interacting CC-type glutaredoxins modulate hyponastic growth in Arabidopsis thaliana', New Phytologist, 221(4), pp. 1906-1918. doi: 10.1111/NPH.15496.

Li, Q., Zheng, J., Li, S., Huang, G., Skilling, S. J., Wang, L., et al. (2017) 'TransporterMediated Nuclear Entry of Jasmonoyl-Isoleucine Is Essential for Jasmonate Signaling', Molecular Plant, 10(5), pp. 695-708. doi: 10.1016/j.molp.2017.01.010.

Li, Z., Luo, X., Ou, Y., Jiao, H., Peng, L., Fu, X., et al. (2021) 'JASMONATE-ZIM DOMAIN proteins engage Polycomb chromatin modifiers to modulate Jasmonate signaling in Arabidopsis', Molecular Plant, 14(5), pp. 732-747. doi: 10.1016/J.MOLP.2021.03.001.

Liu, T., Song, T., Zhang, X., Yuan, H., Su, L., Li, W., et al. (2014) 'Unconventionally secreted effectors of two filamentous pathogens target plant salicylate biosynthesis', Nature Communications 2014 5:1, 5(1), pp. 1-10. doi: 10.1038/ncomms5686.

Liu, Y., Du, M., Deng, L., Shen, J., Fang, M., Chen, Q., et al. (2019) 'Myc2 regulates the termination of jasmonate signaling via an autoregulatory negative feedback loop[open]', Plant Cell, 31(1), pp. 106-127. doi: 10.1105/tpc.18.00405.

Lucas, W. J., Groover, A., Lichtenberger, R., Furuta, K., Yadav, S.-R., Helariutta, Y., et al. (2013) 'The Plant Vascular System: Evolution, Development and FunctionsF', Journal of Integrative Plant Biology, 55(4), pp. 294-388. doi: 10.1111/JIPB.12041.

Macaulay, K. M., Heath, G. A., Ciulli, A., Murphy, A. M., Abell, C., Carr, J. P., et al. (2017) 'The biochemical properties of the two Arabidopsis thaliana isochorismate synthases', Biochemical Journal, 474(10), pp. 1579-1590. doi: 10.1042/BCJ20161069.

Macho, A. P. and Zipfel, C. (2014) 'Plant PRRs and the activation of innate immune signaling', 
Molecular Cell. Cell Press, pp. 263-272. doi: 10.1016/j.molcel.2014.03.028.

Malinovsky, F. G., Fangel, J. U. and Willats, W. G. T. (2014) 'The role of the cell wall in plant immunity', Frontiers in Plant Science. Frontiers Research Foundation, p. 178. doi: 10.3389/fpls.2014.00178.

Mao, G., Meng, X., Liu, Y., Zheng, Z., Chen, Z. and Zhang, S. (2011) 'Phosphorylation of a WRKY transcription factor by two pathogen-responsive MAPKs drives phytoalexin biosynthesis in Arabidopsis', Plant Cell, 23(4), pp. 1639-1653. doi: 10.1105/tpc.111.084996.

Martínez-Abarca, F., Herrera-Cervera, J. A., Bueno, P., Sanjuan, J., Bisseling, T. and Olivares, J. (1998) 'Involvement of Salicylic Acid in the Establishment of the Rhizobium meliloti -Alfalfa Symbiosis', Molecular Plant-Microbe Interactions $\AA$, 11(2), pp. 153-155. doi: 10.1094/MPMI.1998.11.2.153.

Martínez-Medina, A., Appels, F. V. W. and van Wees, S. C. M. (2017) 'Impact of salicylic acid- and jasmonic acid-regulated defences on root colonization by Trichoderma harzianum T78.', Plant signaling \& behavior, 12(8), p. e1345404. doi: 10.1080/15592324.2017.1345404.

Millet, Y. A., Danna, C. H., Clay, N. K., Songnuan, W., Simon, M. D., Werck-Reichhart, D., et al. (2010) 'Innate Immune Responses Activated in Arabidopsis Roots by MicrobeAssociated Molecular Patterns', The Plant Cell, 22(3), pp. 973-990. doi: 10.1105/TPC.109.069658.

Mine, A., Seyfferth, C., Kracher, B., Berens, M. L., Becker, D. and Tsuda, K. (2018) 'The defense phytohormone signaling network enables rapid, high-amplitude transcriptional reprogramming during effector-triggered immunity[OPEN]', Plant Cell, 30(6), pp. 1199-1219. doi: $10.1105 /$ tpc.17.00970.

Mobley, E. M., Kunkel, B. N. and Keith, B. (1999) 'Identification, characterization and comparative analysis of a novel chorismate mutase gene in Arabidopsis thaliana', Gene, 240(1), pp. 115-123. doi: 10.1016/S0378-1119(99)00423-0.

Moisan, K., Raaijmakers, J. M., Dicke, M., Lucas-Barbosa, D. and Cordovez, V. (2021) 'Volatiles from soil-borne fungi affect directional growth of roots', Plant, Cell \& Environment, 44(1), pp. 339-345. doi: 10.1111/PCE.13890.

Moreno, J. E., Shyu, C., Campos, M. L., Patel, L. C., Chung, H. S., Yao, J., et al. (2013) 'Negative feedback control of jasmonate signaling by an alternative splice variant of JAZ10', Plant Physiology, 162(2), pp. 1006-1017. doi: 10.1104/pp.113.218164.

Mou, Z., Fan, W. and Dong, X. (2003) 'Inducers of Plant Systemic Acquired Resistance Regulate NPR1 Function through Redox Changes', Cell, 113(7), pp. 935-944. doi: 10.1016/S0092-8674(03)00429-X.

Murfett, J., Wang, X. J., Hagen, G. and Guilfoyle, T. J. (2001) 'Identification of Arabidopsis histone deacetylase HDA6 mutants that affect transgene expression', Plant Cell, 13(5), pp. 1047-1061. doi: 10.1105/tpc.13.5.1047.

Nakata, M., Mitsuda, N., Herde, M., Koo, A. J. K., Moreno, J. E., Suzuki, K., et al. (2013) 'A bHLH-type transcription factor, ABA-INDUCIBLE BHLH-TYPE TRANSCRIPTION FACTOR/JA-ASSOCIATED MYC2-LIKE1, acts as a repressor to negatively regulate jasmonate signaling in Arabidopsis', Plant Cell, 25(5), pp. 1641-1656. doi: 10.1105/tpc.113.111112.

Nawrath, C. and Métraux, J.-P. (1999) Salicylic Acid Induction-Deficient Mutants of Arabidopsis Express PR-2 and PR-5 and Accumulate High Levels of Camalexin after Pathogen Inoculation, The Plant Cell. Available at: www.plantcell.org.

Ndamukong, I., Abdallat, A. Al, Thurow, C., Fode, B., Zander, M., Weigel, R., et al. (2007) 'SA-inducible Arabidopsis glutaredoxin interacts with TGA factors and suppresses JA- 
responsive PDF1.2 transcription', The Plant Journal, 50(1), pp. 128-139. doi: 10.1111/J.1365313X.2007.03039.X.

Ngou, B. P. M., Ahn, H.-K., Ding, P. and Jones, J. D. G. (2021) 'Mutual potentiation of plant immunity by cell-surface and intracellular receptors', Nature 2021 592:7852, 592(7852), pp. 110-115. doi: 10.1038/s41586-021-03315-7.

Niu, Y., Figueroa, P. and Browse, J. (2011) 'Characterization of JAZ-interacting bHLH transcription factors that regulate jasmonate responses in Arabidopsis', Journal of Experimental Botany, 62(6), pp. 2143-2154. doi: 10.1093/jxb/erq408.

Ohkubo, Y., Tanaka, M., Tabata, R., Ogawa-Ohnishi, M. and Matsubayashi, Y. (2017) 'Shoot-to-root mobile polypeptides involved in systemic regulation of nitrogen acquisition', Nature Plants 2017 3:4, 3(4), pp. 1-6. doi: 10.1038/nplants.2017.29.

Okuma, N., Soyano, T., Suzaki, T. and Kawaguchi, M. (2020) 'MIR2111-5 locus and shootaccumulated mature miR2111 systemically enhance nodulation depending on HAR1 in Lotus japonicus', Nature Communications 2020 11:1, 11(1), pp. 1-13. doi: 10.1038/s41467-02019037-9.

Oome, S., Raaymakers, T. M., Cabral, A., Samwel, S., Böhm, H., Albert, I., et al. (2014) 'Nep1-like proteins from three kingdoms of life act as a microbe-associated molecular pattern in Arabidopsis', Proceedings of the National Academy of Sciences of the United States of America, 111(47), pp. 16955-16960. doi: 10.1073/pnas.1410031111.

Pauwels, L., Barbero, G. F., Geerinck, J., Tilleman, S., Grunewald, W., Pérez, A. C., et al. (2010) 'NINJA connects the co-repressor TOPLESS to jasmonate signalling', Nature, 464(7289), pp. 788-791. doi: 10.1038/nature08854.

Pei, Y., Li, X., Zhu, Y., Ge, X., Sun, Y., Liu, N., et al. (2019) 'GhABP19, a Novel Germin-Like Protein From Gossypium hirsutum, Plays an Important Role in the Regulation of Resistance to Verticillium and Fusarium Wilt Pathogens', Frontiers in Plant Science, 0, p. 583. doi: 10.3389/FPLS.2019.00583.

Pei, Y., Zhu, Y., Jia, Y., Ge, X., Li, X., Li, F., et al. (2020) 'Molecular evidence for the involvement of cotton GhGLP2, in enhanced resistance to Verticillium and Fusarium Wilts and oxidative stress', Scientific Reports 2020 10:1, 10(1), pp. 1-15. doi: 10.1038/s41598-02068943-x.

Pemberton, C. L. and Salmond, G. P. C. (2004) 'The Nep1-like proteins - A growing family of microbial elicitors of plant necrosis', Molecular Plant Pathology. Mol Plant Pathol, pp. 353359. doi: 10.1111/j.1364-3703.2004.00235.x.

Penninckx, A. M. A., Eggermont, K., Terras, F. R. G., Thomma, B. P. H. J., De Samblanx, G. W., Buchala, A., et al. (1996) 'Pathogen-Induced Systemic Activation of a Plant Defensin Gene in Arabidopsis Follows a Salicylic Acid-Independent Pathway', The Plant Cell, 8, pp. 2309-2323. Available at: https://academic.oup.com/plcell/article/8/12/2309/5985185 (Accessed: 22 July 2021).

Pokotylo, I., Kravets, V. and Ruelland, E. (2019) 'Salicylic acid binding proteins (SABPs): The hidden forefront of salicylic acid signalling', International Journal of Molecular Sciences. MDPI AG, p. 4377. doi: 10.3390/ijms20184377.

Poncini, L., Wyrsch, I., Tendon, V. D., Vorley, T., Boller, T., Geldner, N., et al. (2017) 'In roots of Arabidopsis thaliana, the damage-associated molecular pattern AtPep1 is a stronger elicitor of immune signalling than flg22 or the chitin heptamer', PLOS ONE, 12(10), p. e0185808. doi: 10.1371/JOURNAL.PONE.0185808.

Powelson, R. L. and Carter, G. E. (1973) 'Efficacy of soil fumigants for control of Verticillium wilt of potatoes', American Potato Journal 1973 50:5, 50(5), pp. 162-167. doi: 10.1007/BF02853206. 
Pré, M., Atallah, M., Champion, A., De Vos, M., Pieterse, C. M. J. and Memelink, J. (2008) 'The AP2/ERF Domain Transcription Factor ORA59 Integrates Jasmonic Acid and Ethylene Signals in Plant Defense', Plant Physiology, 147(3), pp. 1347-1357. doi: 10.1104/PP.108.117523.

Lo Presti, L., Lanver, D., Schweizer, G., Tanaka, S., Liang, L., Tollot, M., et al. (2015) 'Fungal Effectors and Plant Susceptibility', Annual Review of Plant Biology, 66, pp. 513-545. doi: 10.1146/ANNUREV-ARPLANT-043014-114623.

Pröbsting, M., Schenke, D., Hossain, R., Häder, C., Thurau, T., Wighardt, L., et al. (2020) 'Loss-of-function of CRT1a (Calreticulin) reduces plant susceptibility to Verticillium longisporum in both Arabidopsis thaliana and oilseed rape (Brassica napus )', Plant Biotechnology Journal, p. pbi.13394. doi: 10.1111/pbi.13394.

Qin, J., Wang, K., Sun, L., Xing, H., Wang, S., Li, L., et al. (2018) 'The plant-specific transcription factors CBP60G and SARD1 are targeted by a verticillium secretory protein VDSCP41 to modulate immunity', eLife, 7. doi: 10.7554/eLife.34902.

Qutob, D., Kemmerling, B., Brunner, F., Küfner, I., Engelhardt, S., Gust, A. A., et al. (2006) 'Phytotoxicity and innate immune responses induced by Nep1-like proteins', Plant Cell, 18(12), pp. 3721-3744. doi: 10.1105/tpc. 106.044180.

Ralhan, A., Schöttle, S., Thurow, C., Iven, T., Feussner, I., Polle, A., et al. (2012) 'The vascular pathogen Verticillium longisporum requires a jasmonic acid-independent COI1 function in roots to elicit disease symptoms in Arabidopsis shoots', Plant Physiology, 159(3), pp. 1192-1203. doi: 10.1104/pp.112.198598.

Ratzinger, A., Riediger, N., Tiedemann, A. von and Karlovsky, P. (2009) 'Salicylic acid and salicylic acid glucoside in xylem sap of Brassica napus infected with Verticillium longisporum', Journal of Plant Research 2009 122:5, 122(5), pp. 571-579. doi: 10.1007/S10265-009-02375.

Rekhter, D., Lüdke, D., Ding, Y., Feussner, K., Zienkiewicz, K., Lipka, V., et al. (2019) 'Isochorismate-derived biosynthesis of the plant stress hormone salicylic acid', Science, 365(6452), pp. 498-502. doi: 10.1126/science.aaw1720.

Reusche, M., Thole, K., Janz, D., Truskina, J., Rindfleisch, S., Drübert, C., et al. (2012) 'Verticillium infection triggers VASCULAR-RELATED NAC DOMAIN7-dependent de novo xylem formation and enhances drought tolerance in Arabidopsis', Plant Cell, 24(9), pp. 38233837. doi: $10.1105 /$ tpc. 112.103374 .

Ribnicky, D. M., Shulaev, V. and Raskin, I. (1998) 'Intermediates of salicylic acid biosynthesis in tobacco', Plant Physiology, 118(2), pp. 565-572. doi: 10.1104/pp.118.2.565.

Rich-Griffin, C., Eichmann, R., Reitz, M. U., Hermann, S., Woolley-Allen, K., Brown, P. E., et al. (2020) 'Regulation of Cell Type-Specific Immunity Networks in Arabidopsis Roots', The Plant Cell, 32(9), pp. 2742-2762. doi: 10.1105/TPC.20.00154.

Richmond, T. A. and Bleecker, A. B. (1999) 'A defect in $\beta$-oxidation causes abnormal inflorescence development in arabidopsis', Plant Cell, 11(10), pp. 1911-1923. doi: 10.1105/tpc.11.10.1911.

Růžička, K., Ursache, R., Hejátko, J. and Helariutta, Y. (2015) 'Xylem development - from the cradle to the grave', New Phytologist, 207(3), pp. 519-535. doi: 10.1111/NPH.13383.

Sasaki-Sekimoto, Y., Jikumaru, Y., Obayashi, T., Saito, H., Masuda, S., Kamiya, Y., et al. (2013) 'Basic helix-loop-helix transcription factors JASMONATE-ASSOCIATED MYC2-LIKE1 (JAM1), JAM2, and JAM3 are negative regulators of jasmonate responses in Arabidopsis', Plant Physiology, 163(1), pp. 291-304. doi: 10.1104/pp.113.220129.

Schenkel, D., Maciá-Vicente, J. G., Bissell, A. and Splivallo, R. (2018) 'Fungi Indirectly 
Affect Plant Root Architecture by Modulating Soil Volatile Organic Compounds', Frontiers in Microbiology, O(AUG), p. 1847. doi: 10.3389/FMICB.2018.01847.

Schweizer, F., Fernández-Calvo, P., Zander, M., Diez-Diaz, M., Fonseca, S., Glauser, G., et al. (2013) 'Arabidopsis basic helix-loop-helix transcription factors MYC2,MYC3, andMYC4 regulate glucosinolate biosynthesis, insect performance, and feeding behavior', Plant Cell, 25(8), pp. 3117-3132. doi: 10.1105/tpc.113.115139.

Serrano, M., Wang, B., Aryal, B., Garcion, C., Abou-Mansour, E., Heck, S., et al. (2013) 'Export of salicylic acid from the chloroplast requires the multidrug and toxin extrusion-like transporter EDS5', Plant Physiology, 162(4), pp. 1815-1821. doi: 10.1104/pp.113.218156.

Serrano, M., Coluccia, F., Torres, M., L'Haridon, F. and Métraux, J. P. (2014) 'The cuticle and plant defense to pathogens', Frontiers in Plant Science. Frontiers Research Foundation, p. 274. doi: 10.3389/fpls.2014.00274.

Shabala, S., White, R. G., Djordjevic, M. A., Ruan, Y.-L., Mathesius, U., Shabala, S., et al. (2015) 'Root-to-shoot signalling: integration of diverse molecules, pathways and functions', Functional Plant Biology, 43(2), pp. 87-104. doi: 10.1071/FP15252.

Sheard, L. B., Tan, X., Mao, H., Withers, J., Ben-Nissan, G., Hinds, T. R., et al. (2010) 'Jasmonate perception by inositol-phosphate-potentiated COI1-JAZ co-receptor', Nature, 468(7322), pp. 400-407. doi: 10.1038/nature09430.

Shyu, C., Figueroa, P., de Pew, C. L., Cooke, T. F., Sheard, L. B., Moreno, J. E., et al. (2012) 'JAZ8 lacks a canonical degron and has an EAR motif that mediates transcriptional repression of jasmonate responses in Arabidopsis', Plant Cell, 24(2), pp. 536-550. doi: 10.1105/tpc.111.093005.

Song, S., Qi, T., Huang, H., Ren, Q., Wu, D., Chang, C., et al. (2011) 'The jasmonate-ZIM domain proteins interact with the R2R3-MYB transcription factors MYB21 and MYB24 to affect jasmonate-regulated stamen development in Arabidopsis', Plant Cell, 23(3), pp. 1000-1013. doi: $10.1105 /$ tpc. 111.083089 .

Song, S., Qi, T., Fan, M., Zhang, X., Gao, H., Huang, H., et al. (2013) 'The bHLH Subgroup Illd Factors Negatively Regulate Jasmonate-Mediated Plant Defense and Development', PLoS Genetics, 9(7), p. e1003653. doi: 10.1371/journal.pgen.1003653.

Spoel, S. H., Koornneef, A., Claessens, S. M. C., Korzelius, J. P., Pelt, J. A. Van, Mueller, M. J., et al. (2003) 'NPR1 Modulates Cross-Talk between Salicylate- and JasmonateDependent Defense Pathways through a Novel Function in the Cytosol', The Plant Cell, 15(3), p. 760. doi: 10.1105/TPC.009159.

Stark, C. (1961) 'Das Auftreten der Verticillium-Tracheomykosen in Hamburger Gartenbaukulturen: Ein Beitrag zur Kenntnis ihrer Erreger on JSTOR', Gartenbauwissenschaft, 2, pp. 493-528. Available at: https://www.jstor.org/stable/24136179?refreqid=excelsior\%3Aaad6a20dd34c8b578a503f516 c679f5f (Accessed: 10 July 2021).

Staswick, P. E. and Tiryaki, I. (2004) 'The oxylipin signal jasmonic acid is activated by an enzyme that conjugate it to isoleucine in Arabidopsis W inside box sign', Plant Cell, 16(8), pp. 2117-2127. doi: 10.1105/tpc.104.023549.

Strawn, M. A., Marr, S. K., Inoue, K., Inada, N., Zubieta, C. and Wildermuth, M. C. (2007) 'Arabidopsis Isochorismate Synthase Functional in Pathogen-induced Salicylate Biosynthesis Exhibits Properties Consistent with a Role in Diverse Stress Responses', Journal of Biological Chemistry, 282(8), pp. 5919-5933. doi: 10.1074/JBC.M605193200.

Sun, T., Li, Y., Zhang, Q., Ding, Y., Zhang, Yuelin and Zhang, Yaxi (2015) 'ChIP-seq reveals broad roles of SARD1 and CBP60g in regulating plant immunity', Nature Communications, 6. doi: 10.1038/ncomms10159. 
Sun, T., Busta, L., Zhang, Q., Ding, P., Jetter, R. and Zhang, Y. (2018) 'TGACG-BINDING FACTOR 1 (TGA1) and TGA4 regulate salicylic acid and pipecolic acid biosynthesis by modulating the expression of SYSTEMIC ACQUIRED RESISTANCE DEFICIENT 1 ( SARD1 ) and CALMODULIN-BINDING PROTEIN 60g ( CBP60g )', New Phytologist, 217(1), pp. 344354. doi: $10.1111 / \mathrm{nph} .14780$.

Sun, T., Huang, J., Xu, Y., Verma, V., Jing, B., Sun, Y., et al. (2019) 'Redundant CAMTA Transcription Factors Negatively Regulate the Biosynthesis of Salicylic Acid and NHydroxypipecolic Acid by Modulating the Expression of SARD1 and CBP60g', Molecular Plant, 13(1), pp. 144-156. doi: 10.1016/j.molp.2019.10.016.

Tabata, R., Sumida, K., Yoshii, T., Ohyama, K., Shinohara, H. and Matsubayashi, Y. (2014) 'Perception of root-derived peptides by shoot LRR-RKs mediates systemic N-demand signaling', Science, 346(6207), pp. 343-346. doi: 10.1126/SCIENCE.1257800.

Tada, Y., Spoel, S. H., Pajerowska-Mukhtar, K., Mou, Z., Song, J., Wang, C., et al. (2008) 'Plant Immunity Requires Conformational Charges of NPR1 via S-Nitrosylation and Thioredoxins', Science (New York, N.Y.), 321(5891), pp. 952-956. doi: 10.1126/SCIENCE.1156970.

Tappe, H. (2008) Verticillium longisporum induced gene expression in Arabidopsis thaliana. Georg-August-University. Available at: https://ediss.uni-goettingen.de/handle/11858/00-17350000-0006-B643-B (Accessed: 20 July 2021).

Thines, B., Katsir, L., Melotto, M., Niu, Y., Mandaokar, A., Liu, G., et al. (2007) 'JAZ repressor proteins are targets of the SCFCOI1 complex during jasmonate signalling', Nature, 448(7154), pp. 661-665. doi: 10.1038/nature05960.

Thordal-Christensen, H. (2020) 'A holistic view on plant effector-triggered immunity presented as an iceberg model', Cellular and Molecular Life Sciences. Springer Science and Business Media Deutschland GmbH, pp. 3963-3976. doi: 10.1007/s00018-020-03515-w.

Torrens-Spence, M. P., Bobokalonova, A., Carballo, V., Glinkerman, C. M., Pluskal, T., Shen, A., et al. (2019) 'PBS3 and EPS1 Complete Salicylic Acid Biosynthesis from Isochorismate in Arabidopsis', Molecular Plant, 12(12), pp. 1577-1586. doi: 10.1016/j.molp.2019.11.005.

Truman, W., Sreekanta, S., Lu, Y., Bethke, G., Tsuda, K., Katagiri, F., et al. (2013) 'The CALMODULIN-BINDING PROTEIN60 family includes both negative and positive regulators of plant immunity', Plant Physiology, 163(4), pp. 1741-1751. doi: 10.1104/pp.113.227108.

Tsikou, D., Yan, Z., Holt, D. B., Abel, N. B., Reid, D. E., Madsen, L. H., et al. (2018) 'Systemic control of legume susceptibility to rhizobial infection by a mobile microRNA', Science, 362(6411), pp. 233-236. doi: 10.1126/SCIENCE.AAT6907.

Tsuda, K., Sato, M., Stoddard, T., Glazebrook, J. and Katagiri, F. (2009) 'Network properties of robust immunity in plants', PLoS Genetics, 5(12), p. 1000772. doi: 10.1371/journal.pgen.1000772.

Ulrich, L., Schmitz, J., Thurow, C. and Gatz, C. (2021) 'The jasmonoyl-isoleucine receptor CORONATINE INSENSITIVE1 suppresses defence gene expression in Arabidopsis roots independently of its ligand', The Plant Journal. doi: 10.1111/TPJ.15372.

Vernooij, B., Friedrich, L., Morse, A., Reist, R., Kolditz-Jawhar, R., Ward, E., et al. (1994) 'Salicylic Acid Is Not the Translocated Signal Responsible for Inducing Systemic Acquired Resistance but Is Required in Signal Transduction.', The Plant Cell, 6(7), p. 959. doi: 10.1105/TPC.6.7.959.

De Vos, M., Oosten, V. R. Van, Poecke, R. M. P. Van, Pelt, J. A. Van, Pozo, M. J., Mueller, M. J., et al. (2007) 'Signal Signature and Transcriptome Changes of Arabidopsis During Pathogen and Insect Attack', http://dx.doi.org/10.1094/MPMI-18-0923, 18(9), pp. 923-937. 
doi: 10.1094/MPMI-18-0923.

Wang, G., Hu, C., Zhou, J., Liu, Y., Cai, J., Pan, C., et al. (2019) 'Systemic Root-Shoot Signaling Drives Jasmonate-Based Root Defense against Nematodes', Current Biology, 29(20), pp. 3430-3438.e4. doi: 10.1016/J.CUB.2019.08.049.

Wang, L., Tsuda, K., Sato, M., Cohen, J. D., Katagiri, F. and Glazebrook, J. (2009) 'Arabidopsis CaM binding protein CBP60g contributes to MAMP-induced SA accumulation and is involved in disease resistance against Pseudomonas syringae', PLoS Pathogens, 5(2). doi: 10.1371/journal.ppat.1000301.

Wang, L., Tsuda, K., Truman, W., Sato, M., Nguyen, L. V., Katagiri, F., et al. (2011) 'CBP60g and SARD1 play partially redundant critical roles in salicylic acid signaling', Plant Journal, 67(6), pp. 1029-1041. doi: 10.1111/j.1365-313X.2011.04655.x.

Wang, L., Kim, J. and Somers, D. E. (2013) 'Transcriptional corepressor TOPLESS complexes with pseudoresponse regulator proteins and histone deacetylases to regulate circadian transcription', Proceedings of the National Academy of Sciences of the United States of America, 110(2), p. 761. doi: 10.1073/PNAS.1215010110.

Wang, Y., Li, X., Fan, B., Zhu, C. and Chen, Z. (2021) 'Regulation and function of defenserelated callose deposition in plants', International Journal of Molecular Sciences. MDPI AG, pp. 1-15. doi: 10.3390/ijms22052393.

Wasternack, C. and Hause, B. (2013) 'Jasmonates: Biosynthesis, perception, signal transduction and action in plant stress response, growth and development. An update to the 2007 review in Annals of Botany', Annals of Botany. Oxford Academic, pp. 1021-1058. doi: $10.1093 / \mathrm{aob} / \mathrm{mct} 067$.

Westfall, C. S., Zubieta, C., Herrmann, J., Kapp, U., Nanao, M. H. and Jez, J. M. (2012) 'Structural basis for prereceptor modulation of plant hormones by gh3 proteins', Science, 336(6089), pp. 1708-1711. doi: 10.1126/science.1221863.

Wildermuth, M. C., Dewdney, J., Wu, G. and Ausubel, F. M. (2001) 'Erratum: Isochorismate synthase is required to synthesize salicylic acid for plant defence', Nature, 414(6863), pp. 562565. doi: $10.1038 / 35107108$.

Wu, Y., Zhang, D., Chu, J. Y., Boyle, P., Wang, Y., Brindle, I. D., et al. (2012) 'The Arabidopsis NPR1 Protein Is a Receptor for the Plant Defense Hormone Salicylic Acid', Cell Reports, 1(6), pp. 639-647. doi: 10.1016/J.CELREP.2012.05.008.

Xu, L., Liu, F., Lechner, E., Genschik, P., Crosby, W. L., Ma, H., et al. (2002) 'The SCFCOI1 ubiquitin-ligase complexes are required for jasmonate response in Arabidopsis', Plant Cell, 14(8), pp. 1919-1935. doi: 10.1105/tpc.003368.

Yamasaki, K., Motomura, Y., Yagi, Y., Nomura, H., Kikuchi, S., Nakai, M., et al. (2013) 'Chloroplast envelope localization of EDS5, an essential factor for salicylic acid biosynthesis in arabidopsis thaliana', Plant Signaling and Behavior, 8(4). doi: 10.4161/psb.23603.

Yildiz, I., Mantz, M., Hartmann, M., Zeier, T., Kessel, J., Thurow, C., et al. (2021) 'The mobile SAR signal N-hydroxypipecolic acid induces NPR1-dependent transcriptional reprogramming and immune priming', Plant Physiology, 186(3), p. 1679. doi: 10.1093/PLPHYS/KIAB166.

Yokoo, S., Inoue, S., Suzuki, N., Amakawa, N., Matsui, H., Nakagami, H., et al. (2018) 'Comparative analysis of plant isochorismate synthases reveals structural mechanisms underlying their distinct biochemical properties', Bioscience Reports, 38(2), p. 20171457. doi: 10.1042/BSR20171457.

Yu, C.-W., Liu, X., Luo, M., Chen, C., Lin, X., Tian, G., et al. (2011) 'HISTONE DEACETYLASE6 Interacts with FLOWERING LOCUS D and Regulates Flowering in 
Arabidopsis', Plant Physiology, 156(1), pp. 173-184. doi: 10.1104/PP.111.174417.

Yuan, M., Jiang, Z., Bi, G., Nomura, K., Liu, M., Wang, Y., et al. (2021) 'Pattern-recognition receptors are required for NLR-mediated plant immunity', Nature 2021 592:7852, 592(7852), pp. 105-109. doi: 10.1038/s41586-021-03316-6.

Zander, M., Chen, S., Imkampe, J., Thurow, C. and Gatz, C. (2012) 'Repression of the Arabidopsis thaliana Jasmonic Acid/Ethylene-Induced Defense Pathway by TGA-Interacting Glutaredoxins Depends on Their C-Terminal ALWL Motif', Molecular Plant, 5(4), pp. 831-840. doi: $10.1093 / M P / S S R 113$.

Zarei, A., Körbes, A. P., Younessi, P., Montiel, G., Champion, A. and Memelink, J. (2011) 'Two GCC boxes and AP2/ERF-domain transcription factor ORA59 in jasmonate/ethylenemediated activation of the PDF1.2 promoter in Arabidopsis', Plant Molecular Biology 2011 75:4, 75(4), pp. 321-331. doi: 10.1007/S11103-010-9728-Y.

Zhang, F., Yao, J., Ke, J., Zhang, L., Lam, V. Q., Xin, X. F., et al. (2015) 'Structural basis of JAZ repression of MYC transcription factors in jasmonate signalling', Nature, 525(7568), pp. 269-273. doi: 10.1038/nature14661.

Zhang, F., Ke, J., Zhang, L., Chen, R., Sugimoto, K., Howe, G. A., et al. (2017) 'Structural insights into alternative splicing-mediated desensitization of jasmonate signaling', Proceedings of the National Academy of Sciences of the United States of America. doi: 10.1073/pnas.1616938114.

Zhang, J. and Zhou, J. M. (2010) 'Plant immunity triggered by microbial molecular signatures', Molecular Plant. Oxford University Press, pp. 783-793. doi: 10.1093/mp/ssq035.

Zhang, Y., Fan, W., Kinkema, M., Li, X. and Dong, X. (1999) 'Interaction of NPR1 with basic leucine zipper protein transcription factors that bind sequences required for salicylic acid induction of the PR-1 gene', Proceedings of the National Academy of Sciences, 96(11), pp. 6523-6528. doi: 10.1073/PNAS.96.11.6523.

Zhang, Y. and Li, X. (2019) 'Salicylic acid: biosynthesis, perception, and contributions to plant immunity', Current Opinion in Plant Biology. Elsevier Ltd, pp. 29-36. doi: 10.1016/j.pbi.2019.02.004.

Zhang, Yaxi, Xu, S., Ding, P., Wang, D., Cheng, Y. T., He, J., et al. (2010) 'Control of salicylic acid synthesis and systemic acquired resistance by two members of a plant-specific family of transcription factors.', Proceedings of the National Academy of Sciences of the United States of America, 107(42), pp. 18220-5. doi: 10.1073/pnas.1005225107.

Zheng, X., Koopmann, B. and Tiedemann, A. von (2019) 'Role of Salicylic Acid and Components of the Phenylpropanoid Pathway in Basal and Cultivar-Related Resistance of Oilseed Rape (Brassica napus) to Verticillium longisporum', Plants 2019, Vol. 8, Page 491, 8(11), p. 491. doi: 10.3390/PLANTS8110491.

Zheng, X. Y., Spivey, N. W., Zeng, W., Liu, P. P., Fu, Z. Q., Klessig, D. F., et al. (2012) 'Coronatine promotes pseudomonas syringae virulence in plants by activating a signaling cascade that inhibits salicylic acid accumulation', Cell Host and Microbe, 11(6), pp. 587-596. doi: 10.1016/j.chom.2012.04.014.

Zhou, F., Emonet, A., Tendon, V. D., Marhavy, P., Wu, D., Lahaye, T., et al. (2020) 'Coincidence of Damage and Microbial Patterns Controls Localized Immune Responses in Roots', Cell, 180(3), p. 440. doi: 10.1016/J.CELL.2020.01.013.

Zhou, M., Lu, Y., Bethke, G., Harrison, B. T., Hatsugai, N., Katagiri, F., et al. (2018) 'WRKY70 prevents axenic activation of plant immunity by direct repression of SARD1', New Phytologist, 217(2), pp. 700-712. doi: 10.1111/nph.14846.

Zhu, X., Soliman, A., Islam, M. R., Adam, L. R. and Daayf, F. (2017) 'Verticillium dahliae's 
Isochorismatase Hydrolase Is a Virulence Factor That Contributes to Interference With Potato's Salicylate and Jasmonate Defense Signaling', Frontiers in Plant Science, 0, p. 399. doi: 10.3389/FPLS.2017.00399.

Zhu, Z., An, F., Feng, Y., Li, P., Xue, L., A, M., et al. (2011) 'Derepression of ethylenestabilized transcription factors (EIN3/EIL1) mediates jasmonate and ethylene signaling synergy in Arabidopsis', Proceedings of the National Academy of Sciences, 108(30), pp. 12539-12544. doi: 10.1073/PNAS.1103959108.

Zipfel, C. and Oldroyd, G. E. D. (2017) 'Plant signalling in symbiosis and immunity', Nature. Nature Publishing Group, pp. 328-336. doi: 10.1038/nature22009. 


\section{Appendix}

Table AD1. Primers for Genotyping.

\begin{tabular}{|l|l|l|}
\hline \multirow{3}{*}{ GABIseq_428F12.2 } & Primer ID & Sequence 5'-3' \\
& GK428F12Chr1-LP & GTTCAGTTGCATACAAAGCGCAG \\
\cline { 2 - 3 } & GK428F12Chr1-RP & CGCTGACCAAGGGACACGAGTAC \\
\cline { 2 - 3 } o8409mod & CCATATTGACCATCATACTCATTGC \\
\hline SALK_091556C & SALK_091556C_LP & TTTCATACATGCCTCGAATCTATCC \\
\cline { 2 - 3 } & SALK_091556C_RP & TTTTCGGTCACAAGCCTAATGTTAC \\
\cline { 2 - 3 } SALK_201895C & LBb1.3 & ATTTTGCCGATTTCGGAAC \\
& SALK_201895C_LP & ATATCTATGTAGAGAACCCGCTGC \\
& SALK_201895C_RP & GTGCGTGTATATATAAGCTGTGCC \\
\cline { 2 - 3 } & LBb1.3 & ATTTTGCCGATTTCGGAAC \\
\hline
\end{tabular}

\section{Sequence of pGILDA-VP16-COl1}

CTTGAATTTTCAAAAATTCTTACTTTTTTTTTGGATGGACGCAAAGAAGTTTAATAATCATATTACAT GGCATTACCACCATATACATATCCATATACATATCCATATCTAATCTTACTTATATGTTGTGGAAAT GTAAAGAGCCCCATTATCTTAGCCTAAAAAAACCTTCTCTTTGGAACTTTCAGTAATACGCTTAACT GCTCATTGCTATATTGAAGTACGGATTAGAAGCCGCCGAGCGGGTGACAGCCCTCCGAAGGAAG ACTCTCCTCCGTGCGTCCTCGTCTTCACCGGTCGCGTTCCTGAAACGCAGATGTGCCTCGCGCC GCACTGCTCCGAACAATAAAGATTCTACAATACTAGCTTTTATGGTTATGAAGAGGAAAAATTGGC AGTAACCTGGCCCCACAAACCTTCAAATGAACGAATCAAATTAACAACCATAGGATGATAATGCGA TTAGTTTTTTAGCCTTATTTCTGGGGTAATTAATCAGCGAAGCGATGATTTTTGATCTATTAACAGA TATATAAATGCAAAAACTGCATAACCACTTTAACTAATACTTTCAACATTTTCGGTTTGTATTACTTC TTATTCAAATGTAATAAAAGTATCAACAAAAAATTGTTAATATACCTCTATACTTTAACGTCAAGGA GAAAAAACCCCGGATCAAGGGTGCGATATGAAAGCGTTAACGGCCAGGCAACAAGAGGTGTTTG ATCTCATCCGTGATCACATCAGCCAGACAGGTATGCCGCCGACGCGTGCGGAAATCGCGCAGCG TTTGGGGTTCCGTTCCCCAAACGCGGCTGAAGAACATCTGAAGGCGCTGGCACGCAAAGGCGTT ATTGAAATTGTTTCCGGCGCATCACGCGGGATTCGTCTGTTGCAGGAAGAGGAAGAAGGGTTGC CGCTGGTAGGTCGTGTGGCTGCCGGTGAACCACTTCTGGCGCAACAGCATATTGAAGGTCATTA TCAGGTCGATCCTTCCTTATTCAAGCCGAATGCTGATTTCCTGCTGCGCGTCAGCGGGATGTCGA TGAAAGATATCGGCATTATGGATGGTGACTTGCTGGCAGTGCATAAAACTCAGGATGTACGTAAC GGTCAGGTCGTTGTCGCACGTATTGATGACGAAGTTACCGTTAAGCGCCTGAAAAAACAGGGCA ATAAAGTCGAACTGTTGCCAGAAAATAGCGAGTTTAAACCAATTGTCGTAGATCTTCGTCAGCAGA GCTTCACCATTGAAGGGCTGGCGGTTGGGGTTATTCGCAACGGCGACTGGCTGGAATTCCCATC AGGAGGAGGAGGTTCAGGTGGTGGTGGATCCGGAGGAGGTGGTTCAATTCATATGACGAAAAAC AATTACGGGTCTACCATCGAGGGCCTGCTCGATCTCCCGGACGACGACGCCCCCGAAGAGGCG GGGCTGGCGGCTCCGCGCCTGTCCTTTCTCCCCGCGGGACACACGCGCAGACTGTCGACGGCC CCCCCGACCGATGTCAGCCTGGGGGACGAGCTCCACTTAGACGGCGAGGACGTGGCGATGGCG CATGCCGACGCGCTAGACGATTTCGATCTGGACATGTTGGGGGACGGGGATTCCCCGGGGCCG GGATTTACCCCCCACGACTCCGCCCCCTACGGCGCTCTGGATACGGCCGACTTCGAGTTTGAGC AGATGTTTACCGATGCCCTTGGAATCGACGAGTACGGTGGGGATATCTCTAGGCAGATCACAAGT TTGTACAAAAAAGCAGGCTCCATGGAGGATCCTGATATCAAGAGGTGTAAATTGAGCTGCGTCGC GACGGTTGATGATGTCATCGAGCAAGTCATGACCTATATAACTGACCCGAAAGATCGCGATTCGG CTTCTTTGGTGTGTCGGAGATGGTTCAAGATTGATTCCGAGACGAGAGAGCATGTGACTATGGCG CTTTGCTACACTGCGACGCCTGATCGTCTTAGCCGTCGATTCCCGAACTTGAGGTCGCTCAAGCT TAAAGGCAAGCCTAGAGCAGCTATGTTTAATCTGATCCCTGAGAACTGGGGAGGTTATGTTACTC CTTGGGTTACTGAGATTTCTAACAACCTTAGGCAGCTCAAATCGGTGCACTTCCGACGGATGATT GTCAGTGACTTAGATCTAGATCGTTTAGCTAAAGCTAGAGCAGATGATCTTGAGACTTTGAAGCTA GACAAGTGTTCTGGTTTTACTACTGATGGACTTTTGAGCATCGTTACACACTGCAGGAAAATAAAA ACTTTGTTAATGGAAGAGAGTTCTTTTAGTGAAAAGGATGGTAAGTGGCTTCATGAGCTTGCTCAG CACAACACATCTCTTGAGGTTTTAAACTTCTACATGACGGAGTTTGCCAAAATCAGTCCCAAAGAC 
TTGGAAACCATAGCTAGAAATTGCCGCTCTCTGGTATCTGTGAAGGTCGGTGACTTTGAGATTTT GGAACTAGTTGGGTTCTTTAAGGCTGCAGCTAATCTTGAAGAATTTTGTGGTGGCTCCTTGAATGA GGATATTGGAATGCCTGAGAAGTACATGAATCTGGTTTTTCCCCGAAAACTATGTCGGCTTGGTC TCTCTTACATGGGACCTAATGAAATGCCAATACTATTTCCATTCGCGGCCCAAATCCGAAAGCTG GATTTGCTTTATGCATTGCTAGAAACTGAAGACCATTGTACGCTTATCCAAAAGTGTCCTAATTTG GAAGTTCTCGAGACAAGGAATGTAATCGGAGATAGGGGTCTAGAGGTCCTTGCACAGTACTGTAA GCAGTTGAAGCGGCTGAGGATTGAACGCGGTGCAGATGAACAAGGAATGGAGGACGAAGAAGG CTTAGTCTCACAAAGAGGATTAATCGCTTTGGCTCAGGGCTGCCAGGAGCTAGAATACATGGCG GTGTATGTCTCAGATATAACTAACGAATCTCTTGAAAGCATAGGCACATATCTGAAAAACCTCTGT GACTTCCGCCTTGTCTTACTCGACCGGGAAGAAAGGATTACAGATCTGCCACTGGACAACGGAG TCCGATCTCTTTTGATTGGATGCAAGAAACTCAGACGATTTGCATTCTATCTGAGACAAGGCGGCT TAACCGACTTGGGCTTAAGCTACATCGGACAGTACAGTCCAAACGTGAGATGGATGCTGCTGGG TTACGTAGGTGAATCAGATGAAGGTTTAATGGAATTCTCAAGAGGCTGTCCAAATCTACAGAAGCT AGAGATGAGAGGTTGTTGCTTCAGTGAGCGAGCAATCGCTGCAGCGGTTACAAAATTGCCTTCAC TGAGATACTTGTGGGTACAAGGTTACAGAGCATCGATGACGGGACAAGATCTAATGCAGATGGCT AGACCGTACTGGAACATCGAGCTGATTCCATCAAGAAGAGTCCCGGAAGTGAATCAACAAGGAG AGATAAGAGAGATGGAGCATCCGGCTCATATATTGGCTTACTACTCTCTGGCTGGCCAGAGAACA GATTGTCCAACAACTGTTAGAGTCCTGAAGGAGCCAATATGAGACCCAGCTTTCTTGTACAAAGT GGTTGATGGCCGCATAACTGTCGAGTCGACCTGCAGCCAAGCTAATTCCGGGCGAATTTCTTATG ATTTATGATTTTTATTATTAAATAAGTTATAAAAAAAATAAGTGTATACAAATTTTAAAGTGACTCTTA GGTTTTAAAACGAAAATTCTTATTCTTGAGTAACTCTTTCCTGTAGGTCAGGTTGCTTTCTCAGGTA TAGCATGAGGTCGCTCTTATTGACCACACCTCTACCGGCATGCCGAGCAAATGCCTGCAAATCGC TCCCCATTTCACCCAATTGTAGATATGCTAACTCCAGCAATGAGTTGATGAATCTCGGTGTGTATT TTATGTCCTCAGAGGACAACACCTGTTGTAATCCGTCCGAGCTCCAATTCGCCCTATAGTGAGTC GTATTACAATTCACTGGCCGTCGTTTTTACAACGTCGTGACTGGGAAAACCCTGGCGTTACCCAAC TTAATCGCCTTGCAGCACATCCCCCTTTCGCCAGCTGGCGTAATAGCGAAGAGGCCCGCACCGA TCGCCCTTCCCAACAGTTGCGCAGCCTGAATGGCGAATGGCGCGACGCGCCCTGTAGCGGCGC ATTAAGCGCGGCGGGTGTGGTGGTTACGCGCAGCGTGACCGCTACACTTGCCAGCGCCCTAGC GCCCGCTCCTTTCGCTTTCTTCCCTTCCTTTCTCGCCACGTTCGCCGGCTTTCCCCGTCAAGCTC TAAATCGGGGGCTCCCTTTAGGGTTCCGATTTAGTGCTTTACGGCACCTCGACCCCAAAAAACTT GATTAGGGTGATGGTTCACGTAGTGGGCCATCGCCCTGATAGACGGTTTTTCGCCCTTTGACGTT GGAGTCCACGTTCTTTAATAGTGGACTCTTGTTCCAAACTGGAACAACACTCAACCCTATCTCGGT CTATTCTTTTGATTTATAAGGGATTTTGCCGATTTCGGCCTATTGGTTAAAAAATGAGCTGATTTAA CAAAAATTTAACGCGAATTTTAACAAAATATTAACGTTTACAATTTCCTGATGCGGTATTTTCTCCTT ACGCATCTGTGCGGTATTTCACACCGCATATGATCCGTCGAGTTCAAGAGAAAAAAAAAGAAAAA GCAAAAAGAAAAAAGGAAAGCGCGCCTCGTTCAGAATGACACGTATAGAATGATGCATTACCTTG TCATCTTCAGTATCATACTGTTCGTATACATACTTACTGACATTCATAGGTATACATATATACACAT GTATATATATCGTATGCTGCAGCTTTAAATAATCGGTGTCACTACATAAGAACACCTTTGGTGGAG GGAACATCGTTGGTACCATTGGGCGAGGTGGCTTCTCTTATGGCAACCGCAAGAGCCTTGAACG CACTCTCACTACGGTGATGATCATTCTTGCCTCGCAGACAATCAACGTGGAGGGTAATTCTGCTA GCCTCTGCAAAGCTTTCAAGAAAATGCGGGATCATCTCGCAAGAGAGATCTCCTACTTTCTCCCT TTGCAAACCAAGTTCGACAACTGCGTACGGCCTGTTCGAAAGATCTACCACCGCTCTGGAAAGTG CCTCATCCAAAGGCGCAAATCCTGATCCAAACCTTTTTACTCCACGCGCCAGTAGGGCCTCTTTA AAAGCTTGACCGAGAGCAATCCCGCAGTCTTCAGTGGTGTGATGGTCGTCTATGTGTAAGTCACC AATGCACTCAACGATTAGCGACCAGCCGGAATGCTTGGCCAGAGCATGTATCATATGGTCCAGAA ACCCTATACCTGTGTGGACGTTAATCACTTGCGATTGTGTGGCCTGTTCTGCTACTGCTTCTGCCT CTTTTTCTGGGAAGATCGAGTGCTCTATCGCTAGGGGACCACCCTTTAAAGAGATCGCAATCTGA ATCTTGGTTTCATTTGTAATACGCTTTACTAGGGCTTTCTGCTCTGTCATCTTTGCCTTCGTTTATC TTGCCTGCTCATTTTTTAGTATATTCTTCGAAGAAATCACATTACTTTATATAATGTATAATTCATTA TGTGATAATGCCAATCGCTAAGAAAAAAAAAGAGTCATCCGCTAGGTGGAAAAAAAAAAATGAAAA TCATTACCGAGGCATAAAAAAATATAGAGTGTACTAGAGGAGGCCAAGAGTAATAGAAAAAGAAA ATTGCGGGAAAGGACTGTGTTATGACTTCCCTGACTAATGCCGTGTTCAAACGATACCTGGCAGT GACTCCTAGCGCTCACCAAGCTCTTAAAACGGAATTATGGTGCACTCTCAGTACAATCTGCTCTG ATGCCGCATAGTTAAGCCAGCCCCGACACCCGCCAACACCCGCTGACGCGCCCTGACGGGCTT GTCTGCTCCCGGCATCCGCTTACAGACAAGCTGTGACCGTCTCCGGGAGCTGCATGTGTCAGAG GTTTTCACCGTCATCACCGAAACGCGCGAGACGAAAGGGCCTCGTGATACGCCTATTTTTATAGG TTAATGTCATGATAATAATGGTTTCTTAGGACGGATCGCTTGCCTGTAACTTACACGCGCCTCGTA 
TCTTTTAATGATGGAATAATTTGGGAATTTACTCTGTGTTTATTTATTTTTATGTTTTGTATTTGGATT TTAGAAAGTAAATAAAGAAGGTAGAAGAGTTACGGAATGAAGAAAAAAAAATAAACAAAGGTTTAA AAAATTTCAACAAAAAGCGTACTTTACATATATATTTATTAGACAAGAAAAGCAGATTAAATAGATA TACATTCGATTAACGATAAGTAAAATGTAAAATCACAGGATTTTCGTGTGTGGTCTTCTACACAGA CAAGATGAAACAATTCGGCATTAATACCTGAGAGCAGGAAGAGCAAGATAAAAGGTAGTATTTGT TGGCGATCCCCCTAGAGTCTTTTACATCTTCGGAAAACAAAAACTATTTTTTTCTTTAATTTCTTTTTTT TACTTTCTATTTTTAATTTATATATTTATATTAAAAAATTTAAATTATAATTATTTTTTATAGCACGTGAT GAAAAGGACCCAGGTGGCACTTTTCGGGGAAATGTGCGCGGAACCCCTATTTGTTTATTTTTCTA AATACATTCAAATATGTATCCGCTCATGAGACAATAACCCTGATAAATGCTTCAATAATATTGAAAA AGGAAGAGTATGAGTATTCAACATTTCCGTGTCGCCCTTATTCCCTTTTTTTGCGGCATTTTGCCTT CCTGTTTTTGCTCACCCAGAAACGCTGGTGAAAGTAAAAGATGCTGAAGATCAGTTGGGTGCACG AGTGGGTTACATCGAACTGGATCTCAACAGCGGTAAGATCCTTGAGAGTTTTCGCCCCGAAGAAC GTTTTCCAATGATGAGCACTTTTAAAGTTCTGCTATGTGGCGCGGTATTATCCCGTATTGACGCCG GGCAAGAGCAACTCGGTCGCCGCATACACTATTCTCAGAATGACTTGGTTGAGTACTCACCAGTC ACAGAAAAGCATCTTACGGATGGCATGACAGTAAGAGAATTATGCAGTGCTGCCATAACCATGAG TGATAACACTGCGGCCAACTTACTTCTGACAACGATCGGAGGACCGAAGGAGCTAACCGCTTTTT TTCACAACATGGGGGATCATGTAACTCGCCTTGATCGTTGGGAACCGGAGCTGAATGAAGCCATA CCAAACGACGAGCGTGACACCACGATGCCTGTAGCAATGGCAACAACGTTGCGCAAACTATTAA CTGGCGAACTACTTACTCTAGCTTCCCGGCAACAATTAATAGACTGGATGGAGGCGGATAAAGTT GCAGGACCACTTCTGCGCTCGGCCCTTCCGGCTGGCTGGTTTATTGCTGATAAATCTGGAGCCG GTGAGCGTGGGTCTCGCGGTATCATTGCAGCACTGGGGCCAGATGGTAAGCCCTCCCGTATCGT AGTTATCTACACGACGGGCAGTCAGGCAACTATGGATGAACGAAATAGACAGATCGCTGAGATAG GTGCCTCACTGATTAAGCATTGGTAACTGTCAGACCAAGTTTACTCATATATACTTTAGATTGATTT AAAACTTCATTTTTAATTTAAAAGGATCTAGGTGAAGATCCTTTTTGATAATCTCATGACCAAAATC CCTTAACGTGAGTTTTCGTTCCACTGAGCGTCAGACCCCGTAGAAAAGATCAAAGGATCTTCTTG AGATCCTTTTTTTTCTGCGCGTAATCTGCTGCTTGCAAACAAAAAAACCACCGCTACCAGCGGTGG TTTGTTTGCCGGATCAAGAGCTACCAACTCTTTTTCCGAAGGTAACTGGCTTCAGCAGAGCGCAG ATACCAAATACTGTCCTTCTAGTGTAGCCGTAGTTAGGCCACCACTTCAAGAACTCTGTAGCACC GCCTACATACCTCGCTCTGCTAATCCTGTTACCAGTGGCTGCTGCCAGTGGCGATAAGTCGTGTC TTACCGGGTTGGACTCAAGACGATAGTTACCGGATAAGGCGCAGCGGTCGGGCTGAACGGGGG GTTCGTGCACACAGCCCAGCTTGGAGCGAACGACCTACACCGAACTGAGATACCTACAGCGTGA GCATTGAGAAAGCGCCACGCTTCCCGAAGGGAGAAAGGCGGACAGGTATCCGGTAAGCGGCAG GGTCGGAACAGGAGAGCGCACGAGGGAGCTTCCAGGGGGGAACGCCTGGTATCTTTATAGTCC TGTCGGGTTTCGCCACCTCTGACTTGAGCGTCGATTTTTGTGATGCTCGTCAGGGGGGCCGAGC CTATGGAAAAACGCCAGCAACGCGGCCTTTTTACGGTTCCTGGCCTTTTGCTGGCCTTTTGCTCA CATGTTCTTTCCTGCGTTATCCCCTGATTCTGTGGATAACCGTATTACCGCCTTTGAGTGAGCTGA TACCGCTCGCCGCAGCCGAACGACCGAGCGCAGCGAGTCAGTGAGCGAGGAAGCGGAAGAGC GCCCAATACGCAAACCGCCTCTCCCCGCGCGTTGGCCGATTCATTAATGCAGCTGGCACGACAG GTTTCCCGACTGGAAAGCGGGCAGTGAGCGCAACGCAATTAATGTGAGTTAGCTCACTCATTAG GCACCCCAGGCTTTACACTTTATGCTTCCGGCTCGTATGTTGTGTGGAATTGTGAGCGGATAACA ATTTCACACAGGAAACAGCTATGACCATGATTACCCCAAGCTCGAAATTAACCCTCACTAAAGGG AACAAAAGCTGGTACCGGGCCCCCCCTCGAAATTC 


\section{Acknowledgements}

I would like to express my deep gratitude to everyone who has helped me in undertaking this PhD adventure, in and outside the lab.

Especially, I would like to thank Prof. Gatz for her help over the years. She always pushed me to become a better researcher and develop as a person. Her door and ears were always open to questions and concerns. I would like to thank her for extensively discussing data, giving meticulous feedback in the preparation of presentations and reports and making sure I could always be confident in the final product I presented, for giving me the opportunity to present my work to Thursday-seminar guests and at conferences. The yearly meetings on personal development have helped me identify and accept my own strengths and weaknesses.

I would like to thank Prof. Karlovsky for serving on my Thesis Committee and Dr. Thomas Teichmann for agreeing to be the second reviewer for my thesis on short notice. I would also like to thank Prof. Braus, Prof. Heimel, Dr. Ischebeck and Dr. Wiermer for being part of my examination board.

I would like to thank Corinna for being a constant source of positive feedback, for her input in the regular discussion meetings and for giving me an initial comprehensive crash course in molecular biology when I first started here. I would like to thank her for teaching me about the importance of quality control, for performing informatics-related data processing, and for always taking her time to discuss weird melt curve peaks and other concerns of mine regarding data analysis.

Massive gratitude goes out to Guido and his help over the years. Entering Guido's office is like stepping into a calming, remote mountain monastery where all stress and problems seem to dissolve or at least become manageable. I would like to thank him for taking my, sometimes irrational, fears seriously and showing support in mentally troubling times during the pandemic. I also want to thank him for the many times he helped me out with IT-related problems.

I would like to thank the 'lunch bunch', Aswin, Jelena and Daniel for making my time in Göttingen inside and outside the lab fun and memorable. This includes their significant others, Christina and Josh, who are wonderful people.

Ich möchte mich ganz herzlich bei unseren wundervollen TAs bedanken, die mich nicht nur durch ihre Hilfe im Labor unterstützt haben, sondern auch moralisch immer aufgefangen haben. Vielen Dank an Ronny für die immer gute Stimmung im Labor und die Assistenz bei riesigen Genotypisierungsaktionen. Vielen Dank an Anna, die jahrelang geduldig meine Fragen beantwortet hat und ohne deren Expertise und Unterstützung mein jährlicher Western 
Blot niemals so schön geworden wären. Vielen Dank an Kathi für das Rumärgern mit winzigen Wurzel Pellets und das Pipettieren von unmenschlichen Mengen an Realtimes in einem Tag.

I would like to thank my wonderful lab neighbour and Verticillium-partner Lisa and also Anja for having similar mind-sets and providing great shoulders to cry on or feast on victories together. Thank you to Pascal for knowing almost everything about biochemistry I ever wanted to know and for providing entertaining reading material on his back when walking behind him in the corridor (No thanks, I don't want war). I would like to thank Joachim for being a source of calm in my bi-weekly meetings and for sharing his knowledge on COI1. I would like to thank Irene for being a great role model for never being stressed no matter how many things you are juggling and no matter how frustrating the outcome of an experiment is. Thank you to Isha, Stephan and Ben for the few but nice interactions during day-to-day lab work. I would like to thank my students Dirk, Natalie and Eleni for assistance with my project, mostly with the characterisation of transgenic lines. Having had good teaching moments and more challenging ones helped me develop new skills.

I would like to thank Stuart McMillan who was my lab supervisor during my undergrad thesis work. My thrive to always work in a clean, meticulous and thorough way in the lab stems in large part from his teachings. I would also like to thank Dr. Andrew Desbois, Prof. Anna Amtmann, Dr. Emily Armstrong and all other mentors and teachers I had during my studies for shaping my skills, broadening my horizon and nurturing my fascination for science.

I want to thank all the mental health professionals who I have been lucky to have access to and work with over the years.

I want to thank the numerous progressive and brave women that made it possible for me to grow up in a society where it was never questioned whether or not I could pursue a PhD, among them Elena Lucrezia Cornaro Piscopia, Dorothea Christiane Erxleben, Dorothea Schlözer and Mai Majed Al-Qurashi.

I want to wholeheartedly thank my friends Sarah, Eilidh, Juliane and Jannik whose friendship carried me though many difficult and happy times. I also want to thank my furry friends, foremost Django and Piccolinchen. Special thanks to Pico for providing daily bursts of joy when charging down the corridor.

Deep gratitude goes to my family. I want to thank my mum who always fought by my side like a lioness and made sure I could pursue all the opportunities that lead me to this PhD. I would like to thank my dad for nurturing my curiosity ever since I was a child, entertaining my thoughts and questions on everything and for always enthusiastically engaging in all conversations I started with 'Would you rather...?', 'What if...?' or 'Imagine a world in which...'. If he was still alive today, I am sure he would have followed my PhD discoveries with great interest and 
would have visited often to show me the many places he enjoyed during his studies and PhD time in Göttingen. Thank you to my sister Antonia for teaching me the art of staying organised and proofreading everything from important emails to the final dissertation. Thank you to my sister Stella for understanding my morals and also her many proofreading efforts. Throughout my PhD, my mum and my sisters have only been a phone call away during all crisis and always managed to patch me back together. Thank you to my sister Caroline and her family, my grandparents, Frau Glinka, my extended family and my 'adopted' family in Scotland who besides having little clue what the hell I am doing, always showed me the greatest support and their love.

My love and gratitude go out to my wonderful partner Ally who, despite suffering significant labwork-caused neglect over the years, stuck by my side, kept me sane and enriched my life. Thank you for trying to (mostly unsuccessfully) convince me that failed experiments and weird data were not the end of the world, for making sure I had proper home-cooked meals especially during the final phase of the write up, for always believing in me and for making me smile no matter how bad things were going.

Last but not least I want to thank the three grey hairs I have grown during this PhD, Harriot, Heather and Helena, for reminding me that time is precious and that stressing over everything is not the best use of it. 


\title{
X. Curriculum Vitae
}

\author{
Louisa Ulrich
}

DATE OF BIRTH

$30 / 01 / 1993$

PLACE OF BIRTH

Braunschweig, Germany

NATIONALITY

German

\section{EDUCATION}

$11 / 2017-07 / 2021$

Georg-August Universität - Göttingen, Germany

PhD student in the department of Plant Molecular Biology and Physiology lead by Prof. Gatz as part of the PhD Programme Biology by the Georg-August University School of Science (GAUSS)

PhD Thesis:

Analysis of the JA-lle-independent function of COI1 in Arabidopsis thaliana upon infection with Verticillium longisporum

$08 / 2016-11 / 2017$

University of Glasgow - Glasgow, United Kingdom $1^{\text {st }}$ class Master of Science in Biotechnology

Master dissertation, supervised by Prof. Anna Amtmann, Institute of Molecular Cell and Systems Biology, Molecular Plant Physiology:

Characterisation of Arabidopsis thaliana mutants for genes with potentially cell-type specific functions in root pericycle and xylem parenchyma

09/2012 - 06/2016

University of Stirling - Stirling, United Kingdom

$1^{\text {st }}$ class Bachelor of Science with Honours in Marine Biology

Bachelor dissertation, supervised by Dr. Andrew Desbois, Institute of Aquaculture:

Interaction of Vibrio anguillarum with extracellular traps in the innate immune response of Onchorynchus mykiss

08/2004 - 07/2011

High School Gymnasium Martino Katharineum Braunschweig, Germany

Abitur: 1,9 
$14 / 08 / 17-08 / 09 / 17$

07/06/2016 - 10/07/2016

$10 / 06 / 2013-09 / 07 / 2013$
University of Glasgow, Institute of Molecular Cell and Systems Biology, Department of Plant Science lead by Prof. Amtmann- Glasgow, United Kingdom

Student Laboratory Research Assistant

- Assisted PhD projects on salt stress responses in $A$. thaliana

- Continued research from Master's dissertation
Helmholtz Zentrum für Infektionsforschung -
Braunschweig, Germany
Intern in the Department of Microbial Active Substances

University of Stirling, Bacteriology Laboratory Stirling, United Kingdom

Student Laboratory Research Assistant

- Independently conducted experiments as part of ongoing research of Dr. Andrew Desbois, Experiments aimed to establish optimal timing and dosage of antibiotics against vibriosis in Salmo salar using Galleria mellonella larvae as surrogates

\section{PUBLICATIONS}

Ulrich, L.*, Schmitz, J.*, Thurow, C. and Gatz, C. (2021) 'The jasmonoyl-isoleucine Receptor CORONATINE INSENSITIVE1 suppresses defence gene expression in Arabidopsis roots independently of its ligand', The Plant Journal. doi: 10.1111/TPJ.15372.

${ }^{*}$ Authors contributed equally to this work

\section{PRIZES AND AWARDS}

July 2016

\section{Research Based Learning Prize - University of Stirling}

Awarded for the best overall BSc research project grade within the Institute of Aquaculture in 2016

\section{Best Poster Award}

Awarded by the Germany Botanical Society at the German

Botanical Congress 2019 
X. Curriculum Vitae

Louisa Ulrich

Göttingen, 28/7/21 